\title{
SCIENTIFIC PAPERS SESSIONS
}

\author{
September 21, 2007 Maestrale Room
}

MA01

10.45-12.05 - Advanced imaging modalities: functional

\section{MA01:1:1}

\section{NEUROPLASTICITY ON FUNCTIONAL MRI}

\section{G. Callari ${ }^{1}$, A. Arrigo ${ }^{1}$, C. Serrati ${ }^{2}$}

${ }^{1}$ Neuroradiology of Imperia - Ponente Ligure Neurosciences Department, Imperia, Italy; ${ }^{2}$ Neurology of Imperia - Ponente Ligure Neurosciences Department, Imperia, Italy

Neuroplasticity, consisting in cortical reorganization, is a well known phenomenon that explain how neurological functions can be preserved in cases of brain damage. It can be seen for congenital brain lesion but also in acquired lesion in adult patients particularly if they are involved in an active program of physiotherapy.

We present 2 cases: one is the case of a 42 years old woman with an ArteroVenous Malforation (AVM) located in right primary sensory area without any neurological deficit, where functional MRI (BOLD technique) showed on the sensory task of the left hand, mainly the anterior displacement of the sensory function in the right frontal lobe, and on the motor task of the left hand, mainly the controlateral displacement of the motor function in the left frontal lobe; the second is the case of a 62 years old men that suffered of a stroke involving the left primary sensory and motor area (mainly where the right inferior limb is represented) that had a significant improvement in motor function with an active program of physiotherapy. In that case the functional MRI showed on the motor task of the right foot, mainly the controlateral displacement of the motor function in the right frontal lobe, and on the motor task of the right hand the normal representation in the left motor area anatomically preserved. Our experience confirm that neuroplasticity can be assessed on functional MRI in 1.5 tesla MRI, and can give important informations for location of eloquent areas and to evaluate the response to physiotherapy.
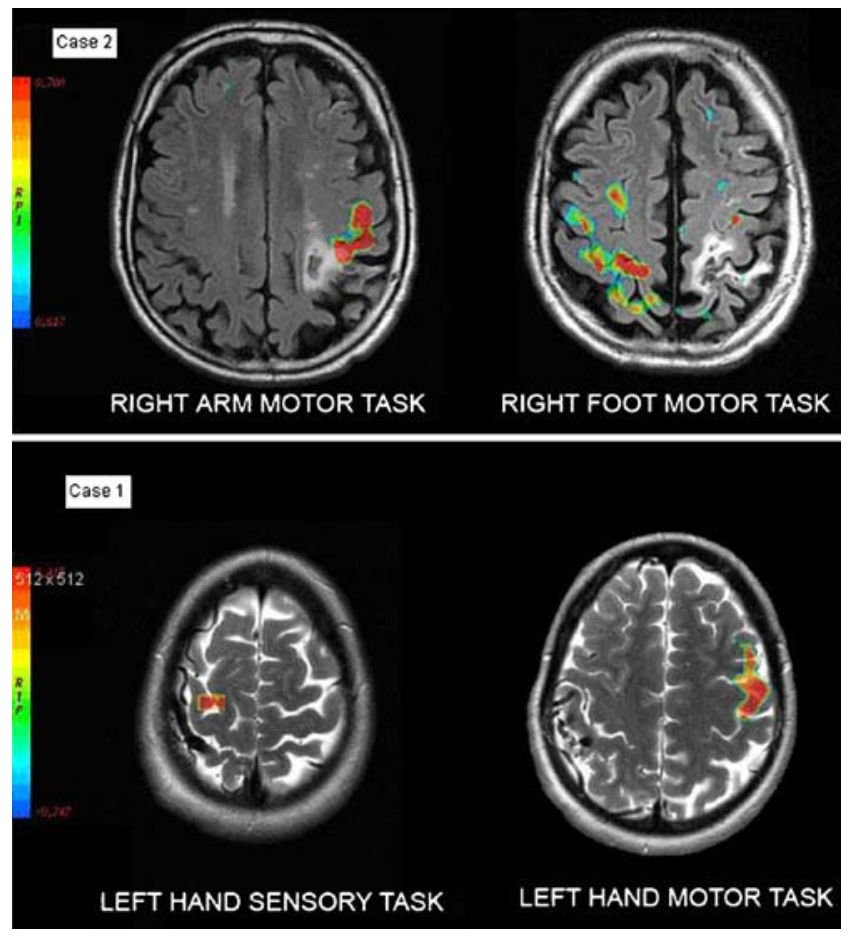

\section{MA01:1:2}

DOES THE TYPE OF BRAIN PATHOLOGY INFLUENCE LANGUAGE PLASTICITY IN NEUROSURGICAL PATIENTS? A FUNCTIONAL-MRI STUDY

M. Caulo ${ }^{1}$, C. Sestieri ${ }^{1}$, C. Briganti ${ }^{1}$,

S. Gaudino ${ }^{1}$, A. Tartaro ${ }^{1}$, C. Colosimo ${ }^{2}$

${ }^{1}$ University of Chieti, Chieti, Italy; ${ }^{2}$ Catholic University, Rome, Italy

\section{INTRODUCTION}

An abnormal organization of cortical language areas has been demontsrated in epileptic patients and in patients with brain tumors, using PET and fMRI. In particular a higher degree of atypical language lateralization has been found in those patients compared to normal subjects. This study aims to demonstrate differences in language plasticity to be correlated with the two different pathological conditions. 


\section{METHODS}

65 right-handed neurosurgical patients of whom 23 with refractory epilepsy (13 men, 10 women; age 17-51) and 42 with brain gliomas (24 men, 18 women; age: 24-78) and 12 age-matched right-handed healthy controls underwent fMRI while performing word and verb generation tasks. The number of active voxels was calculated in each patient and control for the right and left hemisphere and laterality indices were created $[\mathrm{LI}=(\mathrm{L}-\mathrm{R}) /(\mathrm{L}+\mathrm{R})]$. Differences in lateralization were calculated between epilespy, tumor and control groups $(\mathrm{p}<0.01)$. In patients differences in lateralization were correlated with the hemispherical distribution (left or right) of the disease.

\section{RESULTS}

Epilepsy was lesional (18/23) and cryptogenetic (5/23) and the focus was in the right $(15 / 23)$ and in the left hemisphere $(10 / 23)$. Tumors were high and low grade gliomas and were localized in the left hemisphere in 35 patients and in the right in 7 patients. Lateralization for language differed significantly between patients and controls (ANOVA; $\mathrm{p}<0.05$ ). Language was strongly lateralized to the left hemisphere in controls, with a significant higher degree of lateralization than in epilepsy and tumor patients using verb and word generation. Compared to controls, language was significantly less lateralized to the left diseased hemispheres whereas no differences in lateralization were observed in patients with a right diseased hemisphere. The comparison of tumor and epilepsy groups by the side of disease (right or left hemipshere) did not reveal any significant difference in terms of language lateralization. In the left hemisphere tumor group larger tumors caused a stronger shift of language functions to the non-dominant hemisphere.

\section{CONCLUSIONS}

In epilepsy and tumor brains language plasticity is influenced by the side (left or right hemisphere) and not by the type of disease. Larger brain tumors determine a stronger plasticity of language functions.

\section{MA01:1:3}

IS THE CENTER OF MASS (COM) A RELIABLE PARAMETER FOR THE LOCALIZATION OF BRAIN FUNCTION IN FMRI?

G. Fesl ${ }^{1}$, B. Braun ${ }^{1}$, S. Rau ${ }^{2}$, M. Wiesmann ${ }^{1}$, M. Ruge ${ }^{3}$, P. Bruhns ${ }^{1}$, J. Linn ${ }^{1}$, T. Stephan ${ }^{4}$, J. Ilmberger ${ }^{2}$, J. Tonn ${ }^{3}$, H. Brueckmann ${ }^{1}$

1 Neuroradiology, University of Munich, Grosshadern, Munich, Germany; ${ }^{2}$ Physical Medicine and Rehabilitation, University of Munich, Grosshadern, Munich, Germany; ${ }^{3}$ Neurosurgery, University of Munich, Grosshadern, Munich, Germany; ${ }^{4}$ Neurology, University of Munich, Grosshadern, Munich, Germany

\section{PURPOSE}

The center of mass (COM) in functional MRI studies is defined as the center of a cerebral activation cluster. Although the COM is a well-accepted parameter for localizing brain function, the reliability of COMs has not received much attention until now. Our goal was to investigate COM reliability as a function of the thresholding technique, the threshold level, and the type of COM calculation.

METHODS

FMRI was performed on a 1.5 Tesla scanner. Fifteen right-handed subjects were examined repeatedly using a right-sided hand movement task and a tongue movement paradigm. Data analysis was carried out with SPM. Uncorrected, corrected and proportional thresholds (UT, CT and PT) were applied with a range of threshold values. Geometric and T-weighted COMs of primary hand and tongue motor clusters were calculated. The COM variation was evaluated between repeated sessions depending on the different postprocessing setups.

\section{RESULTS}

The hand and tongue COM variations were low for all thresholding techniques at minimal thresholds. Lowest mean variations of hand COMs ranged from $1.6 \mathrm{~mm}$ (PT) to $3.1 \mathrm{~mm}$ (UT). The lowest tongue mean COM variations lay between $7.0 \mathrm{~mm}(\mathrm{PT})$ and $7.9 \mathrm{~mm}(\mathrm{CT})$.

When corrected thresholding was applied, the COM variations deteriorated only slightly with weaker thresholds. At $\mathrm{p}<0.05$ the COM variations were $3.2 \mathrm{~mm}$ for the hand movements and $9.7 \mathrm{~mm}$ for the tongue movements.

The mean hand and tongue COM variations increased remarkably with weaker thresholds when uncorrected thresholding and proportional thresholding were used. Mean COM variations of $7.2 \mathrm{~mm}$ (UT, $\mathrm{p}<0.001)$ and $9.8 \mathrm{~mm}(\mathrm{PT}, \mathrm{p}=0.05)$ were assessed for hand movements. Mean COM variations of $13.6 \mathrm{~mm}(\mathrm{PT}, \mathrm{p}=0.05)$ and $14.4 \mathrm{~mm}$ (UT, $\mathrm{p}<0.001$ ) were calculated for tongue movements. CONCLUSIONS

COM reliability cannot be presupposed; it depends strongly on the individual postprocessing techniques. This should be considered when using COMs for exact localization of brain function.

\section{MA01:1:4}

\section{ICA BASED EEG/FMRI: USE FOR THE DIFFERENTIATION OF EPILEPSY SYNDROMES}

M. Hauf $^{1}$, K. Jann ${ }^{2}$, A. Nirkko ${ }^{3}$, K. Meyer ${ }^{4}$, $\overline{\text { T. Dierks }}^{2}$, T. König ${ }^{2}$, R. Wiest ${ }^{1}$

1 Institute of diagnostic and interventional Neuroradiology, University of Berne, Berne, Switzerland; ${ }^{2}$ Department of Psychiatric Neurophysiology, University of Berne, Berne, Switzerland; ${ }^{3}$ Department of Neurology, University of Berne, Berne, Switzerland; ${ }^{4}$ Bethesda Epilepsy Clinic, Tschugg, Switzerland 


\section{PURPOSE}

Attribution of a specific epilepsy syndrome is crucial for appropriate prognostic and therapeutic concerns and sometimes difficult if exclusively based on clinical, EEG and structural MRI findings. We present data of our series using independent component analysis (ICA) that can be used to localise neuronal epileptogenic activity if applied to EEG/fMRI data and discuss the clinical value in differentiation and localisation of different epilepsy syndromes.

\section{METHODS}

Twenty-four patients, 19 with focal epilepsies (FE) and 5 with idiopathic generalized epilepsies (IGE) were examined with simultaneous 96-channel EEG/fMRI recordings at a $3 \mathrm{~T}$ MR. ICA derived factors coding for time varying epileptiform activity were convolved with a hemodynamic response function to predict the BOLD signal. Voxelwise correlations between the ICApredictors and the BOLD signal were computed. Regions with significant correlations were interpreted being active during the time course of the interictal discharges.

RESULTS

In 4/4 patients with mesial temporal lobe epilepsy (MTLE), in 4/4 with lateral temporal lobe epilepsy (LTLE) and in 5/5 patients with IGE localisation by EEG/fMRI was concordant with the clinical and EEG findings. In 7/11 patients with frontal lobe epilepsies (FLE) at least a lateralisation of the interictal activity was possible. In two patients with FLE fMRI activations did not match the area of interictal spike activity and in two others recordings were interrupted due to seizure occurrence and motion artefacts, respectively. In 9 patients with proper localisation no structural abnormalities were found on structural MRI.

\section{CONCLUSION}

ICA based EEG/fMRI localised the irritative zone properly in $83 \%$ of all patients. The differentiation between IGE and FE was possible in all cases. The results are promising in particularly in MTLE patients and currently, this new approach has been integrated in our evaluation programme for patients undergoing resective epilepsy surgery.

With the support of the Swiss National Foundation (Grant no. $320000-108321 / 1)$

\section{MA01:1:5}

\section{REPRESENTATION OF THE NASAL SKIN AND MUCOSA IN THE HUMAN SOMATOSENSORY CORTEX: FMRI STUDY}

M. Wiesmann, J. May, R. Kopietz, J. Albrecht,

A. Kleemann, V. Schöpf, J. Linn, G. Fesl,

H. Brückmann

Dept. of Neuroradiology, University of Munich, Munich, Germany

\section{INTRODUCTION}

The tactile sensation of the nose is involved in the regulation of both breathing and olfaction. Using the non-invasive method of functional magnetic resonance imaging, the cortical sensory representation of the right nasal skin and mucosa in the primary somatosensory cortex were investigated.

\section{METHODS}

Twenty-four healthy, right-handed subjects were studied using a 1.5 T MR scanner. Tactile stimuli were applied to two parts of the nose: the alar wing of the nose and the lateral nasal mucosa. Images were analyzed using SPM2. The postcentral activation coordinates from the random effects group study were used to define search volumes of $16 \mathrm{~mm}$ radius.

\section{RESULTS AND CONCLUSION}

Local maxima in the postcentral gyrus within these search volumes were found in 18 of 24 subjects when stimulating the alar wing of the nose, and in 23 of the 24 subjects when stimulating the lateral nasal mucosa. Using this method, it was possible to discriminate between the area of representation of the nasal skin and the nasal mucosa in the primary somatosensory cortex. Our results regarding the alar wing of the nose were consistent with the classic 'sensory homunculus' proposed by Penfield. The nasal skin was represented on the postcentral gyrus beneath the thumb and the forehead. In comparison, the nasal mucosa was represented more inferiorly on the postcentral gyrus near the coordinates for the tongue and the pharynx. These results may facilitate the interpretation of fMRI studies of human olfaction.

\section{MA01:1:6}

\section{FUNCTIONAL MRI (FMRI) OF OLFACTORY SYSTEM: PERSISTENCE OF BRAIN ACTIVATION TO ODORS IN KALLMANN SYNDROME}

\author{
$\underline{\text { D. Montanaro }}^{1}$, F. Frijia ${ }^{1}$, N. Vanello ${ }^{2}$, \\ C. Anselmi ${ }^{3}$, F. Vanni ${ }^{2}$, F. Lombardo ${ }^{1}$, F. Bechi ${ }^{4}$, \\ F. Martinelli ${ }^{5}$, R. Canapicchi ${ }^{1}$ \\ ${ }^{1}$ MRI Laboratory, Institute of Clinical Physiology, CNR, Pisa, Italy; \\ 2 Information Engineering Department, University of Pisa, Pisa, \\ Italy; ${ }^{3}$ Center of Cosmetic Science and Technologies, University of \\ Siena, Siena, Italy; ${ }^{4}$ Medical Division, Versilia Hospital, Versilia \\ (LU), Italy; ${ }^{5}$ Radiology Unit, University of Firenze, Firenze, Italy
}

\section{PURPOSE}

Kallmann syndrome (KS) is an inherited disorder characterized by hypogonadotropic hypogonadism and hypo-anosmia, due to an abnormal migration of neurons from olfactory placode to the forebrain and hypothalamus. Structural olfactory abnormalities are well known on MRI, while few data are reported about the residual olfactory functionality. Our goal is to evaluate with fMRI brain activations in response to odors in patients with $\mathrm{KS}$. 


\section{METHOD}

Three patients (one male, 36 yrs; two females, both 14 yrs) affected by KS underwent to olfactometric investigations with a home-made smell test and EEG olfactory test. FMRI was performed with multislices EPI sequences in response to a pure odorous molecule and to a cooling one. Activation areas were derived using correlation analysis performed with the software AFNI. For each olfactory cerebral regions was calculated the frequency of fMRI activations respect to the total administered runs; the different patterns of responses between normal subjects and patients with KS were compared and discussed.

RESULTS

All three patients exhibited various abnormalities of the olfactory brain structures on MRI. At fMRI all the patients showed olfactory activations to each stimulus, in spite of absence of responses at olfactometric tests in two patients and scarce responses in the other one. The patients' responses were lower in each section compared to normal subjects' ones. The activated areas were scattered in few cortical regions, but all located in regions associated with CNS processing of olfactory stimuli.

\section{DISCUSSION AND CONCLUSIONS}

The variable MRI morphological abnormalities don't hinder brain fMRI activations induced by odors, confirming the sense of smell is present also in the so called "anosmic" patients. This could represent a persistence of central imput elaboration, perhaps not strong enough to reach a conscious level.

\section{MA01:1:7}

\section{RIGHT HEMISPHERE DOMINANCE FOR LANGUAGE DEMONSTRATED BY PRE-POST-SURGICAL FMRI AND POST- SURGICAL APHASIA}

\author{
$\underline{\text { P. Vitali }}^{1,3}$, E. Maccagnano ${ }^{1}$, L. D'Incerti ${ }^{1}$, \\ $\overline{\text { C. Marras }}^{2}$, A. Pincherle ${ }^{3}$, R. Spreafico ${ }^{3}$, \\ F. Villani ${ }^{3}$ \\ 1 Neuroradiology, Istituto Neurologico C.Besta, Milano, Italy; \\ 2 Neurosurgery, Istituto Neurologico C.Besta, Milano, Italy; \\ ${ }^{3}$ Neurofisiology and Epilepsy Center, Istituto Neurologico C.Besta, \\ Milano, Italy
}

Atypical language dominance constitute a non-rare finding, particularly when an epileptic focus is in the left hemisphere.

The functional relevance of atypical fMRI activations is still unclear.

\section{CASE REPORT}

In a 31-year-old right-handed man affected by drug-refractory epilepsy associated with a right temporal-parietal cyst and hippocampal sclerosis, language fMRI was performed to plan surgical reduction of the mass-effect of the cyst and to remove the mesial temporal structures.
Two weeks after surgery, the patient presented a global aphasia. CT and MRI showed a chronic subdural haematoma with mass-effect on the right temporal-parietal regions.

An emergency haematoma drainage partially reversed the language deficit.

Despite clear clinical improvement, 6 month after surgery still presented a subtle deficit of both verbal fluency and comprehension; anyway, he remains seizure free.

FMRI METHODS

During the pre-surgical fMRI was performed only an overt verbal fluency task (phonemic fluency, semantic fluency, repetition of non-sense word).

During the post-surgical fMRI were performed overt verbal fluency and overt response naming (auditory response naming, visual response naming, stereotipate phrase completion).

During each task the three conditions were alternated in a six-cycleblock design, and 100 EPI scans $(52 \mathrm{msec}$ TE, $3000 \mathrm{TR}+1 \mathrm{~s}$ interscan time, $2 * 2 * 4 \mathrm{~mm}$ voxel size) were acquired on a 1.5 Tesla Siemens Avanto scanner.

\section{FMRI RESULTS}

The pre-surgical fMRI with verbal fluency showed a bilateral activation in the middle frontal gyrus, with right dominance, and in the right superior temporal sulcus.

fMRI performed 1 month after the haematoma drainage revealed a reduction in the frontal activations (maintaining the right dominance) during verbal fluency; no activation was observed in the temporal lobe, whereas a larger area of activation was observed in the right inferior parietal lobe. The response naming task consistently activated the right superior temporal gyrus in the same area activated in the pre-surgical fMRI.

\section{DISCUSSION}

When a right hemispheric lesion in a right-handed patient causes a language deficit, this condition is referred as crossed aphasia: among the large number of cases reported, only in two study fMRI has been performed (Khateb 2004, Riecker 2004).

The finding of right language representation in a right-handed patient affected by a right focal epilepsy is considered exceedingly rare (Keren 2006, Yuan 2006).

CONCLUSION

Maximal caution is required in neurosurgical planning in the presence of fMRI atypical activations, also when located in a supposedly disfunctional hemisphere.

Figure: Pre-surgical (left) and post-surgical (right) fMRI consistently showed language activation within the right superior temporal sulcus. 


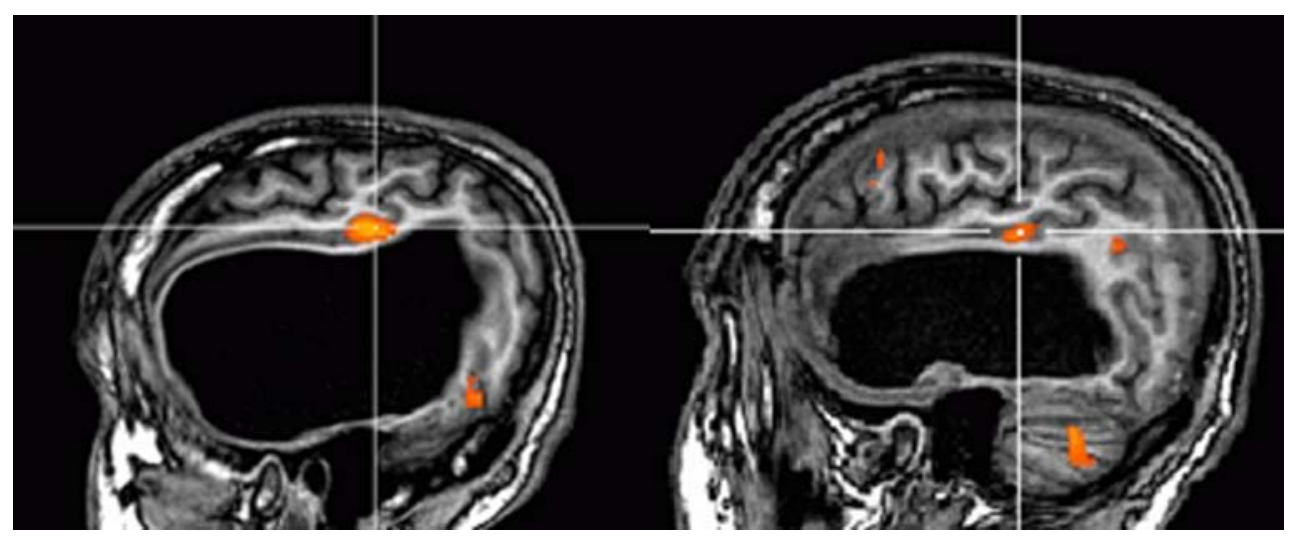

MA01:1:8

\section{VEGETATIVE STATE AND MINIMALLY CONSCIUS \\ PATIENTS: ADVANCED MR ASSESSMENT \\ OF COGNITIVE FUNCTIONS}

M. Maffei, R. Agati, D. Cevolani, L. Albini Riccioli,

S. Battaglia, M. Leonardi

Neuroradiology Department, Bellaria Hospital, Bologna, Italy

\section{BACKGROUND}

Patients in coma following severe acute brain injury may evolve into four clinical states: locked-in syndrome, vegetative state (VS), chronic coma and brain death.

Some patients in VS progressively regain independence through intermediate clinical conditions such as the minimally conscious state (MCS) and confusional state, whereas others evolve into a permanent vegetative state (PVS).

Currently no clinical or instrumental tests are available to predict accurately which patients in VS will regain independence or cognitive function. MR brain activation studies (fMRI) are currently used to try and determine which of these patients could regain consciousness.

\section{PATIENTS AND METHODS}

We used a 3 Tesla magnetic resonance system (Signa ExciteGeneral Electric) to study 13 patients evolving from coma, using the paradigm of familiar voice.

RESULTS AND CONCLUSIONS

fMRI disclosed areas of cortical activation in the temporal regions in 6 patients and in 2 of them in the anterior insular area too.

Concerning the outcome of the patients in which fMRI disclosed areas of cortical activation, we know that two have emerged from VS and regained their independence.

We confirm that MR activation studies may document active cortical networks underlying higher cortical function in the patients in VS and activation of a cortical area in the insular region suggested emotional involvement.

Additional patients and further assessment of the clinical features and follow-up findings in our cohort will establish whether the depiction of areas of cortical activation will serve as a favourable prognostic factor in patients evolving from coma.
MA01:1:9

\section{INCIDENTAL FINDINGS IN RESEARCH FUNCTIONAL MAGNETIC RESONANCE IMAGING: NEUROETHICAL AND PRACTICAL CONSIDERATIONS}

\section{$\underline{\text { A. Server Alonso }}^{1}{ }^{1}$ J. Engh ${ }^{2}$, J. Jensen ${ }^{2}$,} P. Ringen $^{2}$, I. Agartz ${ }^{2}$, P. Nakstad ${ }^{1}$, O. Andreassen ${ }^{2}$

${ }^{1}$ Department of Neuroradiology, Ullevål University Hospital and University of Oslo, Oslo, Norway; ${ }^{2}$ Department of Psychiatry, Ullevål University Hospital and University of Oslo, Oslo, Norway

\section{PURPOSE}

To evaluate the prevalence and severity of incidental findings on brain functional magnetic resonance imaging (fMRI) and to provide standardized procedures for handling these findings.

\section{MATERIAL AND METHODS}

We reviewed the research brain MRI scans of 302 subjects performed from January 2005 to December 2006. All images were reviewed by a neuroradiologist and a confidential report was generated the day after scanning. Incidental findings were classified into four categories: 1) normal or no referral necessary. 2) routine referral; findings not requiring immediate or urgent medical evaluation, but should be reported to the referring physician. 3) urgent referral required within weeks of study for any abnormality that will need further yet nonemergent evaluation. 4) immediate referral required.

RESULTS

Ninety percent $(89,8 \%)$ of the studies were normal or within the no referral category. We detected incidental neuroradiological findings requiring referral in $10,2 \%$ of subjects. Twenty-three subjects $(7,6 \%)$ were classified to have routine referral and eight $(2,6 \%)$ were classified to have urgent referral required. None was classified to have required immediate follow-up evaluation.

We describe the approach that has been used at our institution for handling these findings. First, all MR images are reviewed by a neuroradiologist. Second, although brain research MRI scans are required by several different investigators, the neuroradiological report is always sent to a MD affiliated with the research team (in our case two psychiatrists). Once a week, the neuroradiologist and one of the physicians discuss the need for further imaging. Third, 
one of the two physicians communicate the results to the patient and his/her family doctor.

\section{CONCLUSION}

Unexpected abnormalities are occasionally disclosed on research fMRI . The neuroradiologist and the referring physician or the researcher who run the experiment must be aware of the ethical and practical issues raised by the detection of such findings. We propose standardized guidelines for handling incidental neuroradiological findings.

\section{MA01:1:10}

\section{FUNCTIONAL MAGNETIC RESONANCE IMAGING ETHICS: ARE WE CONCERNED?}

D. Seixas $^{1}$, M. Ayres-Basto ${ }^{2}$

${ }^{1}$ Institute of Histology and Embryology, Faculty of Medicine of Oporto University, Oporto, Portugal; ${ }^{2}$ Neuroradiology Department of Sao Joao Hospital, Oporto, Portugal

\section{PURPOSE}

Neuroethics is a recent discipline that studies the practical and theoretical issues of neuroscience with moral and social consequences. Functional magnetic resonance imaging (fMRI) of the brain raises many questions of difficult answer. The numbers of studies about human cooperation and competition, violence, religion and love have been increasing... Many fMRI studies are done by neuroscientists without any neuroradiological support. What should be done in the case of an incidental finding ( $2 \%$ prevalence) during an fMRI study in a healthy volunteer for research purposes? On the other hand, what to do if a patient presents an abnormal pattern of cortical activation for a certain known task? What is a normal brain? The objective of this review is to bring forth reflection and discussion about ethical issues regarding fMRI.

METHODS

Review of the literature (PubMed and Embase), considering also the authors' experience both on fMRI clinical and research studies.

\section{RESULTS}

FMRI methodology should be known in detail by every neuroscientist involved in these studies, especially its limitations; interpretation of activated areas should be done carefully. Neuroradiologists may be involved in fMRI clinical and research teams. The informed consent for patients and volunteers could have specificities concerning this kind of magnetic resonance imaging technique.

\section{CONCLUSIONS}

Neuroradiologists can have an important role in identifying and solving ethical (and other) issues in fMRI studies. Guidelines are needed to orientate neuroradiologists, clinicians, researchers, patients and volunteers.

\section{MA01:1:11}

3D ANATOMICAL MODELS FOR SURGICAL PLANE: PRELIMINARY EXPERIENCE

V. Dallolio, M. Bonacina

Dept. of Neurosurgery S.Corona Hospital, Pietra Ligure, Italy

\section{OBJECTIVE}

The goal was to develop and realize custom made threedimensional (3-D) anatomical models (life-sized) of specific pathologies of the brain, of the vascular structures and skull base for teaching microneurosurgical anatomy and for operative planning. The neuroimaging research, the development of innovative Rapid Prototyping techniques and the support of modern informatic systems, allow to obtain 3D models, virtual and real models, of anatomical and pathological structures, with very high resolution $(<1 \mathrm{~mm})$; these models are useful tools for the stereoscopic valuation of complex pathologies.

Starting from CT, MRI or Angiografy images, it's possible to realize 3D models of specific pathologies, rapidly, with high resolution, and, above all, for every individual patient ("custom-made" approach). A great experience in cranial reconstruction (cranioplasty custom made) allows the authors to study several Rapid Prototyping techniques realizing 3D models of vascular, cerebral and skull base pathologies. 3D models are a useful tools for:

- $\quad$ surgical plane, combined with Neuronavigation systems;

- real valuation of the geometry, the orientation and the dimensional relationship between pathological and anatomical structures;

- training of young neurosurgeon: 3D models are useful tools for the simulation and for specific (custom made) operative plannig;

- patient education;

- informed consent (to improve the effort to warn the patient about the surgical procedures)

The development of this study, in the future, is the realization of innovative 3D models of vascular structures for fluido-dynamic analyses of specific vascular pathology (MAV, Aneurysm). 
MA02

12.10-13.30 - Advanced imaging modalities: perfusion \& spectroscopy

\section{MA02:2:1}

CEREBRAL BLOOD FLOW BY USING PULSED

ARTERIAL SPIN LABELING IN ELDERLY SUBJECTS WITH WHITE MATTER HYPERINTENSITIES

\author{
A. Bastos-Leite ${ }^{1}$, J. Kuijer ${ }^{2}$, S. Rombouts ${ }^{3}$, \\ E. Sanz-Arigita $^{2}$, E. Van Straaten ${ }^{2}$, A. Gouw ${ }^{2}$, \\ W. Van Der Flier ${ }^{2}$, P. Scheltens ${ }^{2}$, F. Barkhof ${ }^{2}$ \\ 1 Faculdade de Medicina da Universidade do Porto, Oporto, \\ Portugal; ${ }^{2}$ VU University Medical Center, Amsterdam, The \\ Netherlands; ${ }^{3}$ Leiden University Medical Center, Leiden, The \\ Netherlands
}

\section{PURPOSE}

To determine whether there is an association between the amount of white matter hyperintensities (WMH) in elderly subjects and absolute cerebral blood flow (CBF) measurements obtained by means of pulsed arterial spin labeling (PASL) magnetic resonance imaging (MRI).

\section{MATERIAL AND METHODS}

MRI scans of 21 subjects ( 13 women, mean age $=76$, standard deviation $[\mathrm{SD}]=5$ ), stratified for the amount of $\mathrm{WMH}$, from a single center within the multinational Leukoaraiosis and Disability (LADIS) study were investigated. The subjects gave informed consent to be included, and the study was approved by the institutional review board. CBF was determined by using a perfusion-weighted PASL magnetic resonance sequence tailored to an elderly population.

\section{RESULTS}

The mean global CBF, combining gray matter (GM) and white matter (WM), in subjects with WMH was $51.6 \mathrm{ml} / 100 \mathrm{ml} / \mathrm{min}$ $(\mathrm{SD}=10.1)$. Subjects with diffuse confluent $(\mathrm{n}=7) \mathrm{WMH}$ had approximately $20 \%$ lower mean global CBF (43.5 ml/100 ml/min; $\mathrm{SD}=6.3)$ than subjects with punctiform or beginning confluent $(\mathrm{n}=14) \mathrm{WMH}(55.7 \mathrm{ml} / 100 \mathrm{ml} / \mathrm{min} ; \mathrm{SD}=9.2 ; \mathrm{P}<0.01)$. In addition, significant correlations were found between global $\mathrm{CBF}$ and $\mathrm{WMH}$ ( $\mathrm{rs}=-0.54 ; \mathrm{P}<0.05$ ), $\mathrm{WM} \mathrm{CBF}$ and $\mathrm{WMH}$ ( $\mathrm{rs}=$ $-0.52 ; \mathrm{P}<0.05$ ), as well as between GM CBF and WMH (rs= $-0.45 ; \mathrm{P}<0.05)$.

\section{CONCLUSION}

PASL revealed a significant reduction of $\mathrm{CBF}$ in elderly subjects with diffuse confluent WM lesions.
MA02:2:2

\section{HISTOGRAM ANALYSIS OF MR PERFUSION DATA IN PATIENTS WITH MOYAMOYA ARTERIOPATHY AND CORRELATION WITH MRA FINDINGS}

\author{
J. Ederle ${ }^{1}$, J. Thornton ${ }^{1,2}$, J. Grieve ${ }^{1}$, \\ V. Ganesan ${ }^{3}$, M. Brown ${ }^{1}$, H. Jäger ${ }^{1,2}$ \\ 1 Institute of Neurology, UCL, London, United Kingdom; \\ 2 Lysholm Department of Neuroradiology, London, United \\ Kingdom; ${ }^{3}$ Institute of Child Health, UCL, London, United \\ Kingdom
}

\section{AIM}

Analysis of MR perfusion parameters which require an arterial input function is difficult in patients with moyamoya arteriopathy and prone to errors. The aim of this study was to perform histogram analysis of a simple MR perfusion parameter in adult moyamoya patients and to correlate the with the degree of vascular compromise shown on MRA.

\section{METHODS}

12 patients (median age 23 years), ) with moyamoya arteriopathy had dynamic contrast-enhanced MR perfusion imaging and 3D TOF MR angiography. MRA findings were analysed using a previously published 8 point scoring system. Colour maps of time to maximum signal drop (Tmin) were transferred to a Unix Workstation for analysis. ROIs were drawn manually around the vascular territories of the left anterior, right anterior and posterior circulations. Tmin histograms were generated for each ROI and Spearman's rank correlation coefficient calculated respectively for peak height, peak location, SD, skewness, kurtosis versus MRA score.

RESULTS

There was significant negative correlation between the MRA moyamoya score and peak height $(\mathrm{r}=-0.779, \mathrm{p}<0.01)$, standard deviation $(\mathrm{r}=-0.641, \mathrm{p}<0.01)$, and kurtosis $(\mathrm{r}=-0.751, \mathrm{p}<0.01)$. The strongest negative correlation was with skewness $(\mathrm{r}=-0.818, \mathrm{p}<$ $0.01)$. There was no correlation with peak location $(\mathrm{r}=0.241, \mathrm{p}=$ $0.146)$.

Discussion and Conclusion: The negative correlation between MRA score and skewness of the histogram is likely to reflect bolus dispersion and delayed contrast agent wash. Negative correlation between MRA score and peak height is likely to be a sign of a wider range of Tmin caused by bolus dispersion. Our findings show that Tmin histogram metrics correlate well with MRA angiographic findings. This method of analysing brain perfusion data does not require an arterial input function and has the potential for monitoring disease progression and haemodynamic outcomes of revascularisation procedures in patient with moyamoya disease. 


\section{$\mathbf{T}_{\min }$ histograms of $\mathbf{3}$ vascular territories}

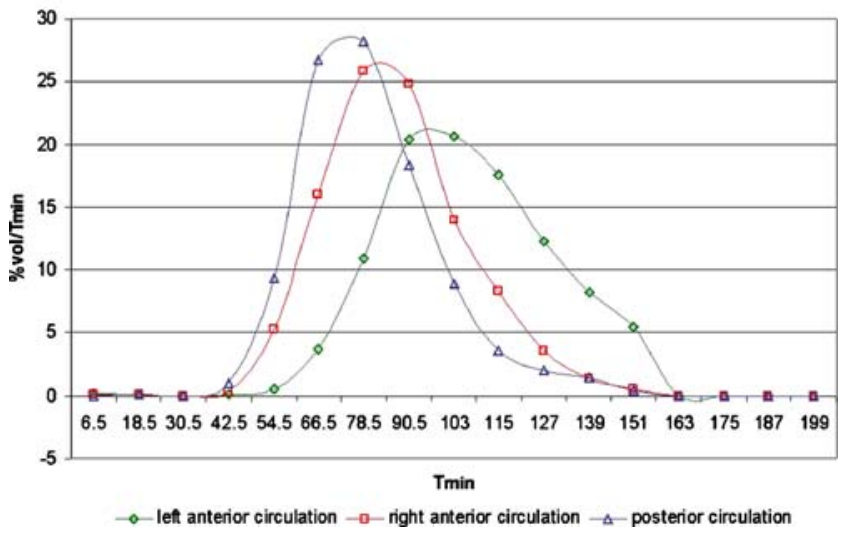

MA02:2:3

PERFUSION WEIGHTED MR IMAGING MAY

DIFFERENTIATE CNS-LYMPHOMA

FROM OTHER HOMOGENEOUSLY ENHANCING BRAIN TUMORS

$\underline{\text { K. Abul-Kasim }}{ }^{1}$, P. Maly ${ }^{1}$, A. Strömbeck ${ }^{1}$, J. Svensson ${ }^{1}$, P. Sundgren $^{2}$

1 University of Lund, Malmö University Hospital, Malmö, Sweden; ${ }^{2}$ University of Michigan Health Systems, Ann Arbor, USA

\section{BACKGROUND}

Every effort should be done to non-invasively differentiate central nervous system lymphoma (CNSL) from glioblastoma multiforme (GBM) and metastases as treatment strategy and cause-specific survival are different. All three types of neoplasia may display homogenous uniform contrast enhancement on CT and MR images and thereby be difficult to differntiate based on conventional imaging alone.

\section{PURPOSE}

To emphasize the value of perfusion weighted images (PWI) in differentiating CNSL from other homogeneously enhancing brain tumors.

\section{MATERIALS AND METHODS}

Retrospective analyse of all conscetive patients presenting with homogeneously enhancing intraaxial tumors on MRI within 2 year long period. All 7 patients ( 3 with CNSL, 3 with GBM, and one with cerebral metastases) have been examined with the same MRsequences including PWI with measurement of rCBV, performed on Siemens scanner ( 6 cases on $3 \mathrm{~T}$ scanner and one case on $1.5 \mathrm{~T}$ scanner).

\section{RESULTS}

GBM and metastases showed significantly increased relative Cerebral Blood Volume (rCBV) while two of lymphomas showed lower $\mathrm{rCBV}$ than normal appearing white matter and the third showed slightly increased $\mathrm{rCBV}$ which was disproportionate to the high degree of contrast enhancement.
Conclusion: PWI may be a valuable method in differentiating CNSL from GBM and metastases in those cases where tumours are homogenously enhancing.

\section{MA02:2:4}

\section{ABSOLUTE QUANTIFICATION OF CEREBRAL MR PERFUSION USING GD-BOPTA}

$\underline{\text { M. Essig }}{ }^{1}$, M. Le Huu ${ }^{1}$, M. Kirchin ${ }^{2}$,

K. Lodemann ${ }^{2}$

1 German Cancer Research Center, Heidelberg, Germany; ${ }^{2}$ Bracco, Milano, Italy

\section{PURPOSE}

Perfusion MRI has proven effective for the assessment of cerebrovascular diseases, brain tumors and dementia. For therapy monitoring an absolute or "semiabsolute" quantification is mandatory. A crucial aspect for the quality the quantification of MR-perfusion is the arterial input function which has to be robust and reliable and the amount of the relative signal reduction, which is the basis for the calculation of $\mathrm{CBV}$ and CBF. Both requirements depend on the concentration and the T2relaxivity of the contrast agents used. The present study was conducted to evaluate the usefulness of Gd-BOPTA for quantitative cerebral perfusion MRI.

MATERIALS \& METHODS

A blinded randomized intra-individual comparative study was conducted in 12 healthy male volunteers and 30 patients with cerebral gliomas. The imaging parameters, slice positioning and contrast media application were standardized. Volunteers were examind with single and double dose, patients with a single dose of Gd-BOPTA.

For a quantitative assessment of the signal time curve, the percentage signal drop and the full width half maximum (FWHM) were assessed in a ROI analysis with ROIs in normal grey and white matter. Beside that, the $\mathrm{rCBV}$ and $\mathrm{rCBF}$ values of gray and white matter were calculated. For the quantitative analysis the image quality of the $\mathrm{rCBV}$ and $\mathrm{rCBF}$ maps in respect of delineation of clinical utility, grey and white matter and basal ganglia delineation were evaluated in an independent off-site assessment.

RESULTS

The studies revealed that a single dose of Gd-BOPTA was sufficient to achieve high quality MR perfusion data. The mean signal drop of approx. $32 \%$ was sufficient to achieve a high quality input function. With the used software package, the calculated $\mathrm{rCBV}$ and $\mathrm{rCBF}$ values of the different ROIs were constant for both dosages within one subject. There were marginal differences in between the subjects. At the qualitative assessment both readers found the single dose images well suited for greywhite matter differentiation and the delineation of the basal ganglia.

In the patient study performed with single dose of Gd-BOPTA all perfusion scans were of excellent diagnostic quality. The qualitative assessment presented a substantial clinical benefit from the perfusion scans in respect of lesion detection and lesion delineation. 


\section{DISCUSSION AND CONCLUSION}

Our results proofed that with the use of the new generation contrast media MultiHance, an absolute quantification and high quality paps of MR perfusion data is possible. The intraindividual values proofed to be constant over time whereas the interindividual differences are objective.

\section{MA02:2:5}

\section{CLINICAL EVALUATION OF VESSEL SIZE IMAGING IN 30 CASES OF HUMAN GLIAL BRAIN TUMOURS}

\author{
I. Mader $^{1}$, T. Breyer ${ }^{1}$, P. Gall ${ }^{2}$, K. Müller ${ }^{3}$, M. Trippel ${ }^{4}$, O. \\ Speck $^{2}$, V. Kiselev ${ }^{2}$ \\ ${ }^{1}$ Section of Neuroradiology, Neurocenter of the Freiburg University \\ Hospital, Freiburg, Germany; ${ }^{2}$ Medical Physics, Freiburg Univer- \\ sity Hospital, Freiburg, Germany; ${ }^{3}$ Dept. of Neuropathology, \\ Neurocenter of the Freiburg University Hospital, Freiburg, Ger- \\ many; ${ }^{4}$ Dept. of Stereotactic Neurosurgery, Neurocenter of the \\ Freiburg University Hospital, Freiburg, Germany
}

\section{INTRODUCTION}

Vessel size imaging is a special form of dynamic susceptibility imaging based on dynamic perfusion measurement during bolus application of a contrast media. Different relaxation curves between spin echoes (SE) and gradient echoes (GE) allow a differentiation of capillaries between 10 and $300 \mathrm{um}$ in diameter. The purpose of this study was to correlate histological grading of human glial brain tumors and the vessel size index (VSI). METHODS

30 patients with glial brain tumors were investigated prior to stereotactic surgery. SE/GE EPI images were acquired during bolus application of gadolinium at a $3 \mathrm{~T}$ whole body system. A region of interest analysis of VSI was performed for normal appearing grey matter (contralateral thalamus) and tumor edge (exclusion of necrosis). A normalisation was performed by dividing the VSI of the tumour edge with the VSI of the contralateral thalamus. Three patients with astrocytoma ${ }^{\circ} \mathrm{II}, 6$ with oligoastrocytoma ${ }^{\circ}$ II, 2 with oligodendroglioma ${ }^{\circ}$ II, 2 with oligodendroglioma ${ }^{\circ} \mathrm{III}, 1$ with oligoastrocytoma ${ }^{\circ} \mathrm{III}, 6$ with astrocytoma ${ }^{\circ} \mathrm{III}$ and 9 patients with glioblastoma multiforme ${ }^{\circ} \mathrm{IV}$ were investigated. All diagnoses were confirmed by histology. A grading for the appearance of the capillaries in the histology was performed. Capillary grad ${ }^{\circ} 1$ was referred to few normal, ${ }^{\circ} 2$ to many normal, ${ }^{\circ} 3$ to many normal and few larger, ${ }^{\circ} 4$ to many atypical and large and ${ }^{\circ} 5$ many atypical very large capillaries being arranged as glomeruli.

RESULTS

Capillary grades ${ }^{\circ} 1$ and 2 were significantly different to ${ }^{\circ} 4$ and ${ }^{\circ} 5$ concerning the VSI index, $\mathrm{p}<0.02$ for all tests. A mis-classification occurred for one glioblastoma and two astrocytoma ${ }^{\circ} \mathrm{III}$ into capillary grade ${ }^{\circ} 2$, and one oligoastrocytoma ${ }^{\circ}$ II into capillary grade ${ }^{\circ} 3$. In the other 26 cases, there was a good agreement between the capillary grade, the histological grading and the VSI.

\section{CONCLUSIONS}

The agreement between VSI indices and capillary grades confirms the theoretical model of VSI in human pathologies. Detailed analysis of the histology showed only few capillaries in the glioblastoma and two astrocytoma ${ }^{\circ} \mathrm{III}$, classified into capillary group 2, who also showed as low VSI index. The oligoastrocytoma ${ }^{\circ}$ II classified into capillary grade ${ }^{\circ} 3$ showed larges capillaries and a higher VSI index. In these cases the mitotic index predominantly accounted for WHO classification. Thus, VSI corresponds to a semiquantitative classification of capillaries, but it is not suitable for a (WHO) classification of brain glial brain tumors. It may play a role for first detection and that of recurrence of well vascularised tumours in the brain.

\section{MA02:2:6}

\section{DOSE REDUCTION IN DYNAMIC PERFUSION CT OF THE BRAIN: EFFECTS OF SCAN FREQUENCY ON CBF, CBV, AND MTT MEASUREMENTS}

$\frac{\text { M. Wiesmann }}{\text { R. Klingebiel }}^{1}{ }^{1}$, V. Schöpf ${ }^{1}$, S. Berg ${ }^{2}$, G. Bohner ${ }^{3}$,

1 Dept. of Neuroradiology, University of Munich, Munich, Germany; ${ }^{2}$ Dept. of Radiology, University Hospital, Basel, Switzerland; ${ }^{3}$ Dept. of Neuroradiology, Charite University Medicine, Berlin, Germany

\section{INTRODUCTION}

Dynamic perfusion CT (PCT) studies of the brain are usually performed using scan frequencies of 1-2 images / sec over a scanning period of 30-40 seconds. Reducing the scan frequency may represent a means to diminish the radiation exposure of the patient if image quality is not compromised. We investigated the influence of the scan frequency on quantitative measurements of cerebral blood flow (CBF), cerebral blood volume (CBV), and mean transit time (MTT).

\section{METHODS}

Eight patients with clinically suspected acute ischemia of one hemisphere underwent dynamic PCT using a 4-row CT scanner, performed on average 3.4 hours after the onset of symptoms. 60 consecutive images per slice were obtained with a scan frequency of 2 images per second. Eight additional data sets were reconstructed with temporal resolutions ranging from 1 image per second to 1 image per 6 seconds. CBF, CBV, and MTT measurements were performed in identical regions of interest for cortical gray matter, white matter, and basal ganglia. Measurements were compared between data sets. Additionally, two neuroradiologists evaluated the PCT images visually to identify areas of abnormal perfusion.

\section{RESULTS}

Images using scan frequencies up to 1 image per 3 seconds were rated to be diagnostically equal to the original data. Even at a scan frequency of 1 image per 5 seconds all areas of infarction were identified. Quantitative differences of CBF and MTT measurements between the original data and the reconstructed data sets were $<10 \%$ using scan frequencies up to 1 image per 3 seconds, 
increasing to $22 \%$ at 1 image per 5 seconds. Differences of CBV measurements were $<10 \%$ at all scan frequencies analyzed.

\section{CONCLUSION}

For dynamic perfusion CT studies of the brain the scan frequency can be reduced to 1 image per 3 seconds without a significant compromise in image quality. In the case of our study protocol this translates into a reduction of radiation dose of the patient of $83 \%$.

\section{MA02:2:7}

\section{BRAIN PERFUSION-CT IN SEVERE HEAD INJURIES:} A PRELIMINARY STUDY

\author{
M. Frigerio $^{1}$, P. Gazzoli ${ }^{2}$, F. Rasulo ${ }^{2}$, \\ $\overline{\text { E. De Peri }}^{2}$, N. Latronico ${ }^{2}$, D. Mardighian ${ }^{3}$, \\ R. Gasparotti ${ }^{3}$ \\ ${ }^{1}$ Cattedra di Radiologia, Brescia, Italy; ${ }^{2} 2^{\circ}$ Servizio di Anestesia e \\ Rianimazione, Brescia, Italy; ${ }^{3}$ Unità operativa di Neuroradiologia, \\ Brescia, Italy
}

\section{PURPOSE}

To assess the clinical impact of brain perfusion CT (PCT) in the management of severe head injuries.

\section{MATERIALS AND METHODS}

The study included 12 patients with severe brain injury (6 subarachnoid haemorrhage secondary to intracranial aneurysm, 5 head trauma, 1 intraparenchymal haemorrhage) hospitalized in the Intensive Care Unit, Spedali Civili, Brescia. Intracranial pressure (ICP), invasive mean arterial blood pressure (MAP), cerebral perfusion pressure (CPP), blood flow velocity in MCA and critical closing pressure (CCP) obtained by transcranial Doppler were simultaneously measured.

PCT studies were preceded by plain CT examination and performed on a 16-row multidetector system (Siemens, Sensation 16, Erlangen, Germany). PCT consisted of two 40-second series of 4 slices (thickness $=6 \mathrm{~mm}$ ) at $1 \mathrm{~s}$ interval during a bolus injection of $50 \mathrm{ml}$ of iodinated contrast medium (c.m.) in a antecubital or jugular vein. $\mathrm{CBF}$ and $\mathrm{CBV}$ absolute values were calculated using the Siemens Syngo perfusion tool. ROIs were manually placed in lenticular nuclei and bilaterally in the hemispheric cortex, trying to avoid the injured brain.

Bivariate correlation was applied to establish significant relations between variables.

All PCT studies were independently reviewed by two neuroradiologists blinded to patients clinical history and outcome.

RESULTS

In the 12 patients mean admission GCS was $6.50 \pm 3.32$ (range 3-12) and mean ICP $14.37 \pm 3.90 \mathrm{mmHg}$ (range 9.34-21.20). Only 2 patients had a mild intracranial hypertension, respectively 19.96 and $21.20 \mathrm{mmHg}$. Mean CPP was $74.23 \pm 7.58 \mathrm{mmHg}$ and mean CCP $25.40 \pm 19.41 \mathrm{mmHg}$. Cortical mean CBF was $65.76 \pm 11.17 \mathrm{ml} / \mathrm{min} /$ $100 \mathrm{~g}$ and mean $\mathrm{CBV}$ was $3.68 \pm 0.52 \mathrm{ml} / 100 \mathrm{~g}$.

A statistically significant correlation was found between cortical $\mathrm{CBF}$ and ICP (Pearson's correlation coefficient $=0.733, \mathrm{p}=0.007$ ), cortical CBV and ICP $(0.721, \mathrm{p}=0.008)$, and CBF and MAP (0.597, $\mathrm{p}=0.040)$. The correlation between $\mathrm{CBV}$ and MAP approached statistical significance $(0.497, \mathrm{p}=0.010)$. No correlations between $\mathrm{CT}$ perfusion parameters and $\mathrm{CPP}$ and $\mathrm{CCP}$ were discovered.

\section{CONCLUSION}

The study has demonstrated a correlation between CBF and CBV values obtained by perfusion $\mathrm{CT}$ quantitative analysis and relevant vital parameters such as ICP and MAP. On the basis of our preliminary results based on a limited number of patients perfusion CT can be proposed as an adjunctive tool for the management of patients with severe head injury.

\section{MA02:2:8}

\section{PRELIMINARY RESULTS WITH 256-MULTISLICE CT: CT ANGIOGRAPHY}

D. San Millan Ruiz ${ }^{1}$, J. Wyse ${ }^{1}$, A. Sasson ${ }^{1}$, P. Gailloud ${ }^{1}$, E. Aldrich ${ }^{2}$, R. Wityk ${ }^{2}$, V. Urrutia ${ }^{2}$, B. Kohler ${ }^{1}$, A. Jones ${ }^{1}$, K. Murphy ${ }^{1}$

${ }^{1}$ Division of Interventional Neuroradiology, Johns Hopkins Medical Institutions, Baltimore, USA; ${ }^{2}$ Department of Neurology, Johns Hopkins Medical Institutions, Baltimore, USA

\section{PURPOSE}

256-multislice CT (256CT) is still an experimental prototype. It acquires $0.5 \mathrm{~mm}$ thickness slices with a craniocaudal coverage of $12.8 \mathrm{~cm}$ allowing 3D data acquisition of the entire brain. It offers isotropic spatial resolution similar to 64-slice CT technology, with much higher temporal resolution offered by scanning the imaging volume (V3) in one second and possibility of performing continuous scanning of a whole brain V3. We present preliminary results of $256 \mathrm{CT}$ angiography (256CTA) studies on a $256 \mathrm{CT}$ prototype (Toshiba Medical Systems Corporation).

\section{MATERIALS AND METHODS}

IRB approval and informed consent was obtained for 11 adult outpatients with cerebro-vascular disorders. Perfusion protocol included: 1) a localizer scan $(80 \mathrm{kV}, 100 \mathrm{~mA}, 1.0 \mathrm{~s} /$ rotation, $0.5 \mathrm{~mm} * 256$, $128 \mathrm{~mm}$, FOV $240 \mathrm{~mm}$ ); 2) volumetric continuous CT scan, initiated $5 \mathrm{~s}$ after contrast product $(\mathrm{CP})$ infusion, initially at $2 \mathrm{~s}$ intervals 15 times, and then at $3 \mathrm{~s}$ intervals 6 times $(80 \mathrm{kV}, 100 \mathrm{~mA}, 1.0 \mathrm{~s} /$ rotation, $0.5 \mathrm{~mm} * 256,128 \mathrm{~mm}$, FOV $240 \mathrm{~mm}) .45 \mathrm{ml}$ of CP were injected at a rate of $6 \mathrm{ml} / \mathrm{s}$ followed by $70 \mathrm{ml}$ of saline solution $(5 \mathrm{ml} / \mathrm{s})$. Total effective dose was $4.5 \mathrm{mSv}$. Pre-contrast, arterial and venous phase images were obtained through a time averaging function where 2 acquired V3 were stacked for each phase. Subtraction images where obtained by subtracting raw-data of the artery and venous phase V3 from the pre-contrast V3. Dynamic MIP and volume-rendered CTA were obtained by sequentially adding the thin slice subtracted V3 images.

\section{RESULTS}

excellent quality arterial and venous angiography was obtained in all cases. Venous and arterial contamination on the arterial and venous phases was resolved by using earlier and later acquired V3. The use of the subtracted technique allowed to remove beam hardening artifacts and visualize meningeal arteries and veins, and 
diploic veins. High resolution 4D dynamic CTA imaging demonstrated the hemodynamics of the intracranial circulation.

\section{CONCLUSION}

256CTA covers a craniocaudal distance of $12.8 \mathrm{~mm}$ which is sufficient to explore the whole brain in most patients. The high spatial and temporal resolutions of 256CTA coupled with the postprocessing acquisition of subtracted and dynamic images render this new technology very promising for studying cerebro-vascular disease.

\section{MA02:2:9}

\section{PRELIMINARY RESULTS WITH 256-MULTISLICE CT: CT PERFUSION}

$\underline{\text { D. San Millian Ruiz }}^{1}$, G. Wyse ${ }^{1}$, A. Sasson ${ }^{1}$, P. Gailloud ${ }^{1}$, E. Aldrich ${ }^{2}$, R. Wityk ${ }^{2}$, V. Urrutia ${ }^{2}$, B. Kohler ${ }^{1}$, A. Jones ${ }^{1}$, K. Murphy ${ }^{1}$

1 Division of Interventional Neuroradiology, Johns Hopkins Medical Institutions, Baltimore, USA; ${ }^{2}$ Department of Neurology, Johns Hopkins Medical Institutions, Baltimore, USA

\section{BACKGROUND}

256-slice CT (256CT) is still an experimental prototype.It acquires $0.5 \mathrm{~mm}$ thickness slices with a craniocaudal coverage of $12.8 \mathrm{~cm}$ allowing 3D data acquisition of the entire brain.It offers isotropic spatial resolution similar to 64-slice CT technology,with much higher temporal resolution offered by scanning the imaging volume (V3) in 1s and allowing for 4D image acquisition.We present preliminary results of $256 \mathrm{CT}$ brain volumetric perfusion (256CTVP) studies on a 256CT prototype (Toshiba Medical Systems Corporation)

\section{MATERIALS AND METHODS}

IRB approval and informed consent was obtained for 11 adult outpatients with cerebro-vascular disorders including extra- and intracranial steno-occlusive disease, Moya-Moya disease, and AVM. Perfusion protocol included a localizer scan and a volumetric continuous $\mathrm{CT}$ scan,initiated $5 \mathrm{~s}$ after contrast product $(\mathrm{CP})$ infusion, initially at $2 \mathrm{~s}$ intervals 15 times, and then at $3 \mathrm{~s}$ intervals 6 times. $45 \mathrm{ml}$ of $\mathrm{CP}$ were injected at a rate of $6 \mathrm{ml} / \mathrm{s}$, followed by an infusion of $70 \mathrm{ml}$ of saline solution at a rate of $5 \mathrm{ml} / \mathrm{second}$. Total effective dose was $4.5 \mathrm{mSv}$.CBF/CBV/MTT maps were generated using modified commercial deconvolution-based software (Toshiba Medical Systems).Volumetric voxel-based analysis allowed to evaluate CT perfusion V3 for each $0.5 \mathrm{~mm}$ voxel, subsequently used to generate $3 \mathrm{D}$ rendering color coding maps.Axial and coronal plane maps were obtained for calculation of $\mathrm{CBF} / \mathrm{CBV} / \mathrm{MTT}$ in regions of interest. There was concomitant acquisition of a non-enhanced head CT, and CTA

\section{RESULTS}

excellent 3D volumetric perfusion maps were obtained.12.8 mm craniocaudal coverage allowed for volumetric perfusion maps of the entire brain. Perfusion MTT/CBV/CBF colored maps were analyzed in the axial and coronal planes, and values were calculated in regions of interests,which correlated with the expected results based on individual pathology and with comparative 16-slice CT and MRI perfusion scans when available

\section{CONCLUSION}

256CTVP is a new CT technology that is feasible in a clinical setting and allows for perfusion mapping of the entire brain,a distinct advantage to perfusion protocols using 16/64-slice CT with smaller sample volumes.Non-enhanced CT, and CTA and perfusion images are generated simultaenously without additional scanning or contrast product.256CT imaging offers a new imaging tool for cerebrovascular disease

\section{MA02:2:10}

\section{GLUTAMINE-GABA METABOLITES MR SPECTROSCOPY:} VARIATION AFTER COGNITIVE ACTIVATION

G. Callari ${ }^{1}$, A. Arrigo ${ }^{1}$, G. Colazzo ${ }^{2}$, A. Vada $^{2}$, C. Serrati ${ }^{2}$

1 Neuroradiology of Imperia - Ponente Ligure Neurosciences Department, Imperia, Italy; ${ }^{2}$ Neurology of Imperia - Ponente Ligure Neurosciences Department, Imperia, Italy

To verify the presence of specific variations of MRS spectrum after cognitive activation in young and old healthy subjects.

\section{INTRODUCTION}

Several studies show different patterns of fMRI activation during neuropsychological tasks in young versus old healthy subjects (Rotte, Age Ageing, 2005). Unfortunately, fMRI activation studies do not give data on neurotransmitters and related metabolites; standard regional spectroscopy studies, instead, have given relevant information in young and old healthy subjects or in Alzheimer patients. Particularly, it is able to detect the glutamateglutamine-GABA metabolites pool (GLX) (Rothman and Shulman, Neuropsychopharmacology, 2002)

\section{METHODS}

Twelve normal subjects have undergone a spectroscopic study using GE SIGNA 1.5 T; proton magnetic resonance spectroscopic (1H-MRS) data were acquired using point resolved spectroscopic (PRESS) localization (echo time $30 \mathrm{~ms}$, repetition time $1500 \mathrm{~ms}$, voxel volume $2 \mathrm{~cm} 3$ ). Region of interest was positioned manually in mesial temporal region of dominant (Annett test) hemisphere; The ratio between NAA and $\alpha$ GLX or $\beta \gamma$ GLX was analyzed in basal condition and after cognitive activation with verbal fluency and digit span.

\section{RESULTS}

Due to movement artefacts, only nine subjects $(5<40$ y.o.; 4 between 60 and 70 y.o.; global mean age 46,3 +/-20,7) were analyzed. "T" and "Mann-Withey" tests were used. A significant difference $(\mathrm{p}<0.05)$ was found between basal and post-activation spectrum $(\beta \gamma \mathrm{GLX})$; a trend of positive correlation was found between age and $\delta(\%) \beta \gamma$ GLX $(r=0,44)$.

DISCUSSION

Activation spectroscopy, even if performed with scanners commonly used in clinical practice, can detect variations in the glutamineGABA metabolites $(\beta \gamma \mathrm{GLX})$. It could also allow some cautious 
physiological considerations: even in a easy cognitive task, the old normal subjects seem to activate neural networks more than young (Fridriksson, J Speech Lang Hear Res, 2006; Serrati and Baron, Stroke 2001). Our data could stimulate to verify the usefulness of this protocol as diagnostic tool and during neuropharmacological treatments.

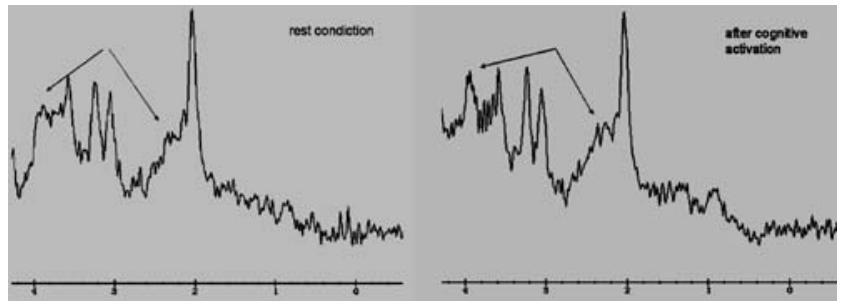

MA02:2:11

\section{PROTON MAGNETIC RESONANCE SPECTROSCOPY IN THE EVALUATION OF HEREDITARY SPASTIC PARAPLEGIA TYPE SPG4}

$\underline{\text { A. Server Alonso }}^{1}{ }^{1}$, A. Erichsen ${ }^{2}$, N. Landrø ${ }^{3}$, C. Tallaksen ${ }^{2}$, P. Nakstad ${ }^{1}$

${ }^{1}$ Department of Neuroradiology, Ullevål University Hospital and University of Oslo, Oslo, Norway; ${ }^{2}$ Department of Neurology, Ullevål University Hospital and University of Oslo, Oslo, Norway; ${ }^{3}$ Department of Psychology, University of Oslo, Oslo, Norway

\section{PURPOSE}

The aim of this study was to determine the metabolite pattern in patients with hereditary spastic paraplegia type SPG4 (SPG4-HSP) compared with those of normal control subjects, and to further investigate the association of proton magnetic resonance spectroscopy (MRS) results with cognitive impairment.

\section{MATERIAL AND METHODS}

We included 8 patients from 4 families with genetically confirmed SPG4-HSP and 8 healthy age- and sex-matched controls. Singlevoxel MRS of the brain was performed using a 1,5 T MRI system in all patients and control subjects. Fully relaxed short-echo time proton MR spectra (TR/TE 1500/30 ms) were recorded using a point resolved spectroscopy sequence; volumes of interest were located in the left frontal white matter and in the left precentral gyrus. Peaks analyzed were those of N-acetyl aspartate (NAA), creatine $(\mathrm{Cr})$, choline-containing compounds (Cho) and myoinositol (Ins); the following ratios were calculated for both locations: NAA/Cr, NAA/Cho, $\mathrm{Cho} / \mathrm{Cr}$ and $\mathrm{Ins} / \mathrm{Cr}$.

\section{RESULTS}

The ratio of Cho/Cr in SPG4-HSP patients showed a significant reduction $(p=0,047)$ in the motor cortex compared with controls, suggesting reduced membrane turnover as result of cell loss.

In addition $\mathrm{NAA} / \mathrm{Cr}$ tended to decline and $\mathrm{Ins} / \mathrm{Cr}$ to increase in SPG4-HSP patients, but no significant differences were found between the two groups. It was noteworthy the correlation of the ratio of $\mathrm{Cho} / \mathrm{Cr}$ with cognitive impairment

CONCLUSION
MRS proved to be a useful investigational tool for detection of metabolite abnormalities in areas of brain that appeared normal on MRI. Cho/Cr could be a surrogate marker of neurodegenerative process in patients with SPG4-HSP.

\section{MA03}

15.25-16.30 - Session III - Stroke I

\section{MA03:3:1}

\section{PERFUSION CT IN TIME: ITS CLINICAL IMPORTANCE}

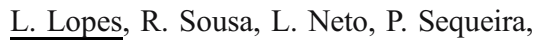

J. Campos

Santa Maria Hospital, Lisbon, Portugal

\section{BACKGROUND AND PURPOSE}

Perfusion CT (PCT) provides information about cerebral perfusion with differentiation of irreversibly damaged brain tissue from reversibly impaired tissue at risk. PCT is thus an important tool in the selection of patients for thromboyitic therapy. Based on the quantification of hemodinamic parameters such as mean transit time (MTT), cerebral blood flow (CBF) and cerebral blood volume (CBV) the potencial recuperation ratio (PRR) may be calculated. The purpose of this study is to quantify the existing PRR in a 24 hour time frame and evaluate the importance of MTT normalisation.

METHODS

Thirty patients with middle cerebral artery (MCA) ischemic stroke underwent serial PCT in the acute and subacute stage. Clinical assessment and outcome were measured using modified National Institute of Health Stroke Scale (mNIHSS).

RESULTS

The PRR decreased with time within the 24 hours of clinical onset. However, within this time window it demonstrated considerable variation. Patients that demonstrated a MTT normalisation on the second study, had better clinical outcome.

\section{CONCLUSION}

The quantification of PRR may provide an insight to the extent of penumbra, which may be of paramount importance in the therapeutic decision. Values of MTT may help to monitor therapeutic response.

\section{MA03:3:2}

\section{CHANGES IN PERFUSION PARAMETERS OF DOUBLE CT PERFUSION STUDIES AS PREDICTOR OF EARLY MORTALITY IN ISCHEMIC STROKE}

$\underline{\text { S. Langner }}^{1}$, S. Stumpe ${ }^{1}$, A. Khaw ${ }^{2}$, M. Kirsch ${ }^{1}$, N. Hosten ${ }^{1}$

${ }_{1}^{1}$ Institute for Diagnostic Radiology and Neuroradiology, ErnstMoritz-Arndt University, Greifswald, Germany; ${ }^{2}$ Department of Neurology, Ernst-Moritz-Arndt University, Greifswald, Germany 


\section{PURPOSE}

Stroke is the third leading cause of death worldwide and one of the main causes of disability in most countries. Most important ist the knowledge of predictors of mortality in ischemic stroke, which will help to identify patients at a higher risk of fatal outcome and will allow the introduction of optimal therapeutic strategies in stroke units. The aim of the study was to evaluate the predictive value of changes in perfusion CT parameters within 48 hours for early neurologic outcome.

\section{METHOD}

In a prospective study 31 consecutive patients with clinical signs of acute ischemic stroke were evaluated. All examinations were performed in a 16 row MSCT (Sensation 16, SIEMENS). CT perfusion was performed on admission and repeated within 48 hours ( 2 slices, $12 \mathrm{~mm}$ slice thickness, $1 \mathrm{scan} / \mathrm{s}$ for $40 \mathrm{~s}, 80 \mathrm{kV}$, $209 \mathrm{mAs}, 40 \mathrm{ml}$ contrast agent, flow $5,9 \mathrm{~mL} / \mathrm{s}$ ). Perfusion data was analyzed using the Neuroperfusion software of the vendor. Perfusion maps for rCBV, rCBF and TTP were calculated for each hemisphere and examinations. Relative changes in perfusion parameters were calculated. Neurological assessment was done on admission and daily until discharge using NIHSS. Spearman correlation and ROC curves were used for statistical analysis.

RESULTS

Perfusion CT was diagnostic in all patients. Improvement or stable NIHSS was observed in $26 / 31$ patients $(74,2 \%)$ and worsening of symptoms in $7 / 31$. In 17 patients an increase in $\mathrm{rCBF}$ correlated with a stable or improved NIHSS on discharge $(\mathrm{p}=0,037)$ with a cut-off value of 3,76 in percental change. Increased $\mathrm{rCBV}$ correlated with improved NIHSS in $13 / 31$ patientens $(p=0,049)$. There was no correlation for TTP.

\section{CONCLUSION}

Changes in $\mathrm{rCBF}$ of repeated $\mathrm{CT}$ perfusion within 48 hours have a high predictive value for early neurologic outcome.

\section{MA03:3:3}

DYNAMIC CT PERFUSION WITH ACETAZOLAMIDE CHALLENGE IN ISCHEMIC ADULT MOYAMOYA DISEASE: CORRELATION WITH ANGIOGRAPHIC STAGES

\author{
$\underline{\text { H. Kim }}^{1}$, S. Kim ${ }^{1}$, Y. Shin ${ }^{2}$, H. Nam ${ }^{3}$ \\ ${ }^{1}$ Department of Diagnostic Radiology, Ajou University, School of \\ Medicine, Suwon, South Korea; ${ }^{2}$ Department of Neurosurgery, \\ Ajou University, School of Medicine, Suwon, South Korea; \\ ${ }^{3}$ Department of Neurology, Ajou University, School of Medicine, \\ Suwon, South Korea
}

\section{PURPOSE}

To validate the dynamic CT perfusion (CTP) with acetazolamide challenge for the evaluation of the hemodynamic status in ischemic adult Moyamoya disease comparing with angiographic findings.

METHODS

12 adult patients with ischemic Moyamoya diseases and six agematched normal controls were enrolled in this study. Baseline hemodynamic parameters and the percent changes of cerebral blood volume (CBV), cerebral blood flow (CBF), and mean transit time (MTT) derived from CTP with acetazolamide challenge were calculated in 36 hemispheres. The regions of interest were placed in the basal ganglia (BG), 3 cortical regions of major arterial territories and watershed zone, and periventricular white matter of each hemisphere. From the angiographies, we classified the degrees of internal carotid artery (ICA) stenosis based on modified Suzuki's stage and determined the presence of basal Moyamoya vessels (BMV). Baseline hemodynamic parameters and their percent changes in each region were compared with the angiographic findings.

RESULTS

In cortices distal to the occluded ICA, the angiographic stages were significantly correlated with the percent change of $\mathrm{CBF}$ $(\mathrm{r}=0.82, \mathrm{p}<0.05)$, the percent change of MTT $(\mathrm{r}=0.78, \mathrm{p}<0.05)$, and the baseline MTT $(r=0.72, p<0.05)$. Baseline parameters except for MTT were not correlated with the angiographic stages. The extensive BMV hemispheres exhibited a significantly lower percent change of CBF and a significantly higher percent change of MTT and baseline MTT than in diminished BMV hemispheres and controls $(\mathrm{p}<0.05)$.

CONCLUSION

The CTP with acetazolamide challenge is feasible in the evaluation of hemodynamic impairment in ischemic adult moyamoya disease and the percent changes of CBF and MTT are significantly correlated with the angiographic stages.

CLINICAL RELEVANCE

CT perfusion can be used for the evaluation of cerebral vascular reserve following acetazolamide challenge in the adult patients with ischemic Moyamoya diseases.

\section{MA03:3:4}

\section{IS THERE ANY RESTING HEMODYNAMIC PARAMETER REFLECTING CEREBRAL AUTOREGULATORY RESPONSE?: EVALUATION WITH CT PERFUSION AFTER ACETAZOLAMIDE ADMINISTRATION}

$\underline{\text { H. Kim, S. Kim }}$

Ajou University School of Medicine, Suwon, South Korea

\section{BACKGROUND and PURPOSE}

It has been reported that resting CT perfusion (CTP) parameter including cerebral blood volume $(\mathrm{CBV})$ and mean transit time (MTT) can reflect cerebral autoregulatory response in chronic cerebrovascular steno-occlusive diseases. we assessed if any relationship between resting hemodynamic parameters and cerebrovascular reserve (CVR) by using CTP with acetazolamide challenge.

\section{METHODS}

25 patients with a unilateral severe internal carotid artery stenosis were enrolled. The resting hemodynamic parameters including CBV and MTT were obtained by CTP before acetazolamide administration. To estimate the CVR, the cerebral blood flow (CBF) response to acetazolamide was assessed by measuring 
baseline CBF and CBF 15 minutes after acetazolamide administration. The ratio of the $\mathrm{CBF}$ value on the occlusive side to the that on the contralateral side was calculated as the asymmetry index (AI), and the proportion of the acetazolamide-challenged AI to the baseline AI was defined as the regional reactivity index. Multivariate analysis was used to test the independent predictive value of the resting parameters with respect to the change of $\mathrm{CBF}$ during acetazolamide administration.

RESULTS

The MTT ratio in the poor CVR group was significantly $(\mathrm{P}<.05)$ larger than that in the normal CVR group and a significant correlation $(\mathrm{r}=-0.79, \mathrm{P}<.05)$ was found between the MTT ratio and the regional reactivity index. The $\mathrm{CBV}$ ratio was not significantly different between the poor and the normal CVR groups. Multivariate analysis revealed that the MTT ratio was significantly and independently associated with the percent change of $\mathrm{CBF}$ during acetazolamide administration, whereas the CBV ratio was not.

CONCLUSION

The MTT ratio and the percent change of $\mathrm{CBF}$ to acetazolamide on CTP were well correlated, suggesting that measurement of the MTT by using a CTP can be used as an index of CVR.

\section{MA03:3:5}

\section{THE VALUE OF PERFUSION CT USING ABSOLUTE BLOOD FLOW VALUES TO DIFFERENTIATE PENUMBRA FROM INFARCTION IN THE ACUTE STROKE SETTING}

F. Borny ${ }^{1}$, P. Seynaeve ${ }^{1}$, H. Pottel ${ }^{3}$, H. Verschelde ${ }^{2}$, J. Goyens ${ }^{2}$, P. Bourgeois ${ }^{2}$, G. Meersman ${ }^{2}$, E. Brugman ${ }^{1}$

1 Dept of Radiology AZ Groeninge Loofstraat 43, Kortrijk, Belgium; ${ }^{2}$ Dept of Neurology AZ Groeninge Loofstraat 43, Kortrijk, Belgium; ${ }^{3}$ Interdisciplinary research center,KUL, Etienne sabbelaan 53, Kortrijk, Belgium

\section{PURPOSE}

Perfusion CT (PCT) allows rapid identification of salvageable tissue in patients presenting with acute stroke symptoms, rendering it indispensable in patient selection for thrombolysis. Several parameters have been described to differentiate penumbra from infarction. In this study we evaluate the accuracy of the use of the combination of a prolonged relative mean transit time (rMTT) and the use of absolute values of cerebral blood flow $(\mathrm{CBF})$.

\section{METHODS AND MATERIALS}

A total of 53 consecutive patients who presented with stroke-like symptoms in the emergency department were selected for inclusion in this study. Of these 53 patients, 11 patients were lost for follow-up.
A native $\mathrm{CT}$, a cine PCT and a post contrast $\mathrm{CT}$ was performed using a 64 slice $\mathrm{CT}$. Absolute $\mathrm{CBF}$ values were obtained using the deconvolution method. A CBF cut off value of $10 \mathrm{ml} / 100 \mathrm{~g} / \mathrm{min}$ in a region of a prolonged relative MTT region was interpreted as infarction, while the presence of more than $10 \mathrm{ml} / 100 \mathrm{~g} / \mathrm{min}$ in a region of prolonged rMTT was considered penumbra. The results were compared with the MR images obtained 24-72 hours later. The use of rMTT to evaluate the presence of infarction resulted in a sensitivity of $75 \%$, a specificity of $77,3 \%$ and an accuracy of $76,2 \%$. Using a $\mathrm{CBF}<10 \mathrm{ml} / 100 \mathrm{~g} / \mathrm{min}$ in a region of prolonged rMTT as a means to diagnose the presence of an infarct gives the following results: sensitivity $60 \%$, specificity $100 \%$ and an accuracy of $81 \%$.

CONCLUSION:

The use of a CBF cutoff value of $10 \mathrm{ml} / 100 \mathrm{~g} / \mathrm{min}$ in a region with a prolonged relative MTT is a very specific parameter to delineate a region of infarction. In our series a $100 \%$ specificity was reached. The relative poor sensitivity (60\%)is mainly due to the partial coverage of the brain since most missed infarcts were small and localized above the CT perfusion imaging slab.

\section{MA03:3:6}

\section{MAGNETIC RESONANCE IMAGING IN ACUTE STROKE: FALSE NEGATIVE ON DIFFUSION WEIGHTED IMAGING}

G. Callari ${ }^{1}$, A. Arrigo ${ }^{1}$, M. Furlan ${ }^{2}$, C. Serrati ${ }^{2}$

1 Neuroradiology of Imperia - Ponente Ligure Neurosciences Department, Imperia, Italy; ${ }^{2}$ Neurology of Imperia - Ponente Ligure Neurosciences Department, Imperia, Italy

Sensitivity on detecting an ischemic lesion in patients with an acute stroke can improve using MRI and particularly performing DWI sequences in axial and coronal plane with $b$ values 1000 and 2000.

In our Center we performed an early MRI for acute stroke (up to 3 hours from symptoms onset) in 11 patients. In 2 cases with a clinical suspicious of a brain stem lesion, the early examination in the acute phase did not show a clear lesion on a biplane DWI b1000 and b2000, lesion that has been seen on a 24-48 hours follow up MRI.

Our experience confirms that false negative MRI for detecting an acute ischemic lesion should be considered, particularly when a posterior fossa lesion is clinically suspected, the patient is in good clinical condition (NIHSS score less than 4) and the time from symptoms onset to scan is less than 3 hours (Chalela et al, The Lancet - January 2007). 


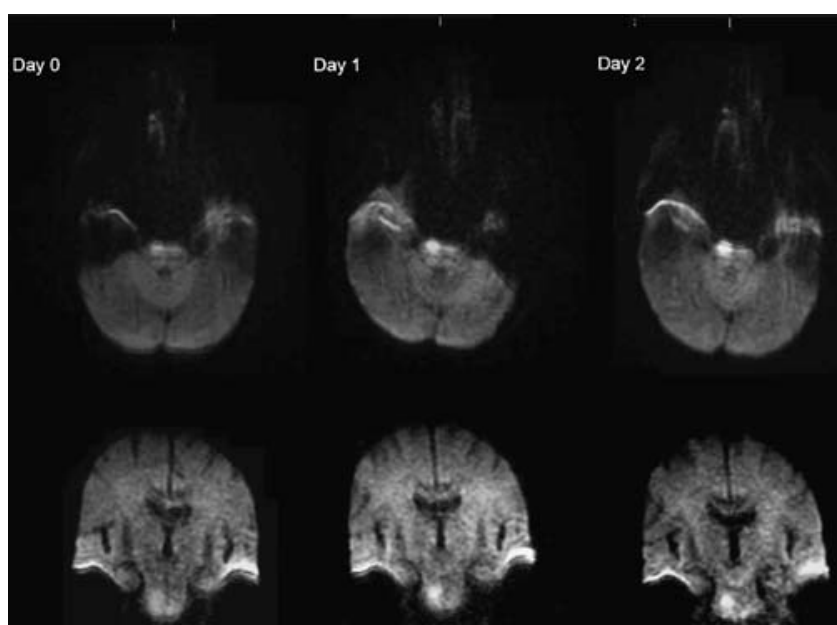

MA03:3:7

DIFFUSION-MR ANGIOGRAPHY MISMATCH. A NOVEL DIAGNOSTIC APPROACH TO ISCHEMIC PENUMBRA

$\underline{\text { A. Rovira }}{ }^{1}$, C. Auger ${ }^{1}$, A. Leon ${ }^{1}$, L. Frascheri ${ }^{1}$, J. Munuera ${ }^{1}$ Hospital Vall d'Hebron, Barcelona, Spain

\section{PURPOSE}

We investigated the diagnostic properties of a mismatch between the presence of a proximal vessel occlusion (ICA and/or M1 segment) and the extension of the diffusion-weighted imaging (DWI) lesion (DWI-MRA mismatch) for predicting the presence of a diffusion-perfusion mismatch (DPM).

MATERIALS AND METHODS

Consecutive acute stroke patients referred to our department for MR imaging evaluation. Inclusion criteria for this study were: (a) Presence of an acute infarct within the MCA territory demonstrated at initial or follow-up imaging; and (b) MR examination carried out within the first 6 hours after symptoms onset, including DWI, PWI (time-to-peak maps) and MR angiography (MRA). Volume measurements of the extension of the tissue abnormality on DWI and timeto-peak (TTP) maps were done with a manual tracing technique. DWI-MRA mismatch was defined as presence of an occlusion of the ICA and/or M1 segment associated with an ischemic volume on DWI of ¡Ü100 mL. Positive DPM was defined as PWIvol DWIvol / DWIvol $\times 100>20 \%$ associated with an ischemic volume on DWI of ¡Ü100 mL.

\section{RESULTS}

204 patients were included in this study (115 female, mean age: 71.7 years). Arterial occlusion was identified in 192 patients (94.1\%). Mean DWI volume was $47 \mathrm{~mL}$ (range 0 to $366 \mathrm{~mL}$ ), while mean PWI volume was $188 \mathrm{~mL}$ (range 0 to $439 \mathrm{~mL}$ ).

125 patients $(61.3 \%)$ had a positive DWI-MRA mismatch and 169 patients $(82.8 \%)$ had a positive DPM. The diagnostic properties of presence of DWI-MRA mismatch with regard to presence of DPM showed a sensitivity of $72.8 \%$, a specificity of $94.3 \%$ and an accuracy of $76.4 \%$.

\section{CONCLUSION}

DWI-MRA mismatch predicts the presence of DPM with very high specificity. This novel approach to ischemic penumbra may simplify the MRI diagnostic workup for selecting candidates for thrombolytic therapy in a high percentage of cases.

\section{MA03:3:8}

TIME COURSE AND CORRELATION OF DIFFUSION-WEIGHTED MR IMAGING AND CT IN ACUTE ISCHEMIA WITH RAT MODEL

\section{H. Seo ${ }^{1}$, D. $\mathrm{Na}^{2}$, J. Kim ${ }^{2}$, K. Chang ${ }^{2}$}

1 Dongguk university international hostpital, Goyang, South Korea; ${ }^{2}$ Seoul National university school of Medicine, Seoul, South Korea

\section{PURPOSE}

To evaluate the time courses of the diffusion-weighted MR imaging (DWI) signal, the apparent diffusion coefficient (ADC) value and the CT density in acute ischemic brain parenchyma, and to seek how the changes of DWI signal and ADC value correlate with the CT density change by a rat model.

\section{MATERIALS AND METHODS}

Among total 29 SD rats, 11 rats completed the scheduled CT and DWI studies for 9 hours with acute left middle cerebral artery (MCA) ischemia by the intraluminal suture occlusion method. CT and DWI studies were acquired at 1, 3, 5, 7 and 9 hours after MCA occlusion. Relative values of DWI signal (rDWI), ADC value ( $\mathrm{rADC}$ ) and $\mathrm{CT}$ density ( $\mathrm{rCT}$ ) were obtained by dividing the values of ischemic lesion by the value of normal contralateral hemisphere. The time courses of each mean relative values were evaluated. The correlation of $\mathrm{rDWI}$ and $\mathrm{rADC}$ to $\mathrm{rCT}$ were evaluated.

RESULTS

$\mathrm{rCT}$ showed a continuous linear decrease with time after ischemia $(\mathrm{r}=$ $0.82, \mathrm{P}<0.01)$ and $\mathrm{rDWI}$ showed a continuous linear increase $(\mathrm{r}=$ $0.56, \mathrm{P}<0.01$ ), whereas the decrease of $\mathrm{rADC}$ was almost completed at 1 hour and did not differ from the $\mathrm{ADC}$ at later stage after 1 hour. $\mathrm{rDWI}$ and $\mathrm{rCT}$ were correlated with statistical significance $(\mathrm{r}=-0.59$, $\mathrm{P}<0.01)$, whereas $\mathrm{rADC}$ and $\mathrm{rCT}$ were not correlated $(\mathrm{P}=0.76)$.

\section{CONCLUSION}

Unlike ADC value, DWI signal in acute cerebral ischemia increases with time and correlates with CT density. It means that the signal change of DWI after 1 hour is influenced by net water uptake in ischemic edema rather than by restricted water diffusion and DWI may be used as the predictor of hemorrhage after thrombolysis like CT. 


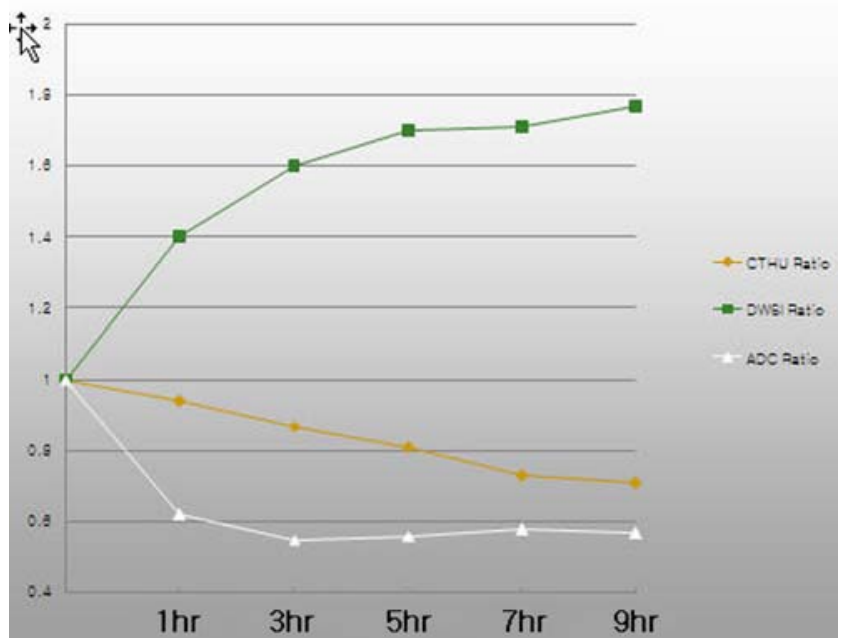

MA03:3:9

\section{WHAT IS THE VALUE OF CCT IMAGING IN PATIENTS WITH TRANSIENT ISCHEMIC ATTACK?}

$\frac{\text { S. Ulmer }}{\text { C. Koch }}^{2}$, C. Wiese ${ }^{2}$, O. Jansen ${ }^{1}$,

1 Section of Neuroradiology, Department of Neurosurgery, University Hospital of Schleswig-Holstein, Kiel, Germany;

${ }^{2}$ Institute of Neuroradiology, University Hospital of SchleswigHolstein, Luebeck, Germany

\section{BACKGROUND}

A transient ischemic attack (TIA) is defined by neurological symptoms lasting less than an hour without evidence of infarction, however, these patients have an increased risk to develop a major stroke. Cranial CT (CCT) as the widely available imaging modality of choice is usually initially performed to rule out other causes of this neurological deterioration. To justify exposure to radiation by $\mathrm{CT}$ this modality should be sensitive enough to depicted underlying causes. The frequency and origin of other causes presenting as TIA has not been addressed to date.

\section{MATERIALS AND METHODS}

We reviewed all CCT-requests within one year of patients presenting with TIA. CT scans, emergency notes, charts and other performed imaging modalities were reviewed. Cases that turned out to be a seizure with Todd paralysis rather than a TIA and other causes that would require immediate emergency imaging (i.e. anticoagulated patients with head trauma or acute hypertensive dysregulation) were excluded.

\section{RESULTS}

From 3580 requests for a CCT, $350(9.8 \%)$ were performed because of transient neurological symptoms. Only 205 of these patients $(5.7 \%)$ presented with a TIA lasting less than one hour. Five cases $(2.4 \%)$ had a pathological finding. One infarction was diagnosed on CCT that turned out to be artificial in the follow up. Two infarctions were found, of which one showed already hemorrhagic transformation. We detected one metastasis (lung cancer) and one meningeoma. In the remaining group without pathological findings, 4 infarctions were found on MRI.

\section{CONCLUSIONS}

Strokes can present as TIA especially when they affect clinically "silent" regions. 50\% of pathological findings were completely missed on CCT, which is dispensable in TIA especially with regard to the applied radiation exposure. Instead a sufficient cerebrovascular imaging should be performed.

\section{MA04}

16.55-18.00 - Session IV - Stroke II

\section{MA04:4:1}

\section{COMBATSTROKE - A PROSPECTIVE RANDOMISED EUROPEAN STUDY TO COMPARE INTRAVENOUS AND ENDOVASCULAR TREATMENT IN ACUTE ANTERIOR CIRCULATION STROKE}

\author{
C. Ozdoba ${ }^{1}$, H. Mattle ${ }^{2}$, G. Schroth ${ }^{1}$, \\ $\overline{\text { K. Decker }}^{3}$ \\ 1 Institute of Diagnostic and Interventional Neuroradiology, \\ Inselspital / University of Bern, Bern, Switzerland; \\ ${ }^{2}$ Department of Neurology, Inselspital / University of Bern, Bern, \\ Switzerland; ${ }^{3}$ Decker Consulting GmbH, Rotkreuz, Switzerland
}

\section{PURPOSE}

Preliminary results indicate that the outcome after endovascular recanalising procedures is superior to the results achieved by intravenous treatment in acute (up to three hours) anterior circulation stroke. This study will prospectively compare the two therapies in a large-scale randomised trial.

\section{MATERIAL AND METHODS}

In the course of the European "Research Framework Programme 7", 45 centres from 13 European countries formed a consortium that plans to perform a prospective study that will compare intravenous and endovascular treatment for acute stroke in 1186 patients over a five-year-period. The project named COMBATSTROKE (Collaborative European Multi-center Basic Science and Clinical Approach in the Treatment of Stroke) will, in addition, test new devices and procedures for the endovascular treatment arm according to standardised criteria, and it will develop and test a new device for localised hypothermia of the brain in endovascular stroke treatment. RESULTS

At the time of abstract submission (04/2007), the project proposal is still under consideration; the European Commission's decision will not be known before July/August. Yet, the study coordinators do not want to miss the opportunity to inform the European neuroradiological community about a project proposal that has attracted Europe-wide interest. Furthermore, the proposal is officially supported by the European Society of Neuroradiology. The talk will discuss the aims and purpose of the project in detail, 
present the project participants, and will discuss COMBATSTROKE's organisation and public activities.

\section{MA04:4:2}

\section{ASSOCIATION BETWEEN INTRA- AND EXTRA-CRANIAL VASCULAR STENOSIS/OCCLUSION WITH CEREBRO VASCULAR LESIONS: RETROSPECTIVE EVALUATION OF 734 PATIENTS STUDIED WITH MR IMAGING}

\author{
A. Tartaro ${ }^{1}$, P. Mattei ${ }^{1}$, S. Gaudino ${ }^{1}$, \\ $\overline{\text { M. Caulo }}^{1}$, M. Kirchin ${ }^{2}$ \\ ${ }^{1}$ University of Chieti, Chieti, Italy; ${ }^{2}$ Bracco Imaging SpA, \\ Milano, Italy
}

\section{PURPOSE}

To evaluate the association between cerebral vascular lesions and severe steno-occlusive disease of the intracranial and/or extracranial blood vessels.

\section{METHODS}

734 patients undergoing supra-aortic contrast enhanced (CE) MRA, intracranial MRA and brain CE MRI were retrospectively evaluated. Patients with prior TEA were excluded, as were patients undergoing follow-up or emergency examinations. MRI of the extra- and intracranial arterial vasculature and brain was performed at $1.5 \mathrm{~T}$. CE MRA and MRI were performed with $0.1 \mathrm{mmol} / \mathrm{kg}$ Gd-BOPTA. All images were evaluated by two experienced radiologists in consensus. The extra- and intracranial vasculature was evaluated for the presence and grade of stenosis/occlusion using NASCET criteria. Brain images were evaluated for leuko-araiosis, lacunar infarction, territorial infarction, border zone infarction and, in elderly patients, arteriolosclerotic leucoencephalopathy (ALE). Association between intra-/extra-cranial vascular disease and cerebrovascular lesions was determined by gender (487 men [69.8 \pm 8.0 years]; 247 women $[71.0 \pm 7.4$ years]) and age $(<65$ years $[n=166] ; 65-72$ years $[\mathrm{n}=279] ;>72$ years $[\mathrm{n}=289])$.

RESULTS

A total of 823 exams were performed. Significant association was noted between leuko-araiosis presence and older ( $>72$ years) age $(\mathrm{OR}=3.10 ; \mathrm{CI}=1.40-6.86)$. Lacunar infarction was not associated with any factor. Territorial infarction was associated with gender $(\mathrm{OR}=0.62 ; \mathrm{CI}=0.44-0.88)$ and with stenosis/occlusion of the left ICA $(\mathrm{OR}=1.36 ; \mathrm{CI}=0.98-1.90)$ and left vertebral artery $(\mathrm{OR}=$ 2.85; $\mathrm{CI}=1.06-7.67)$. Border zone infarction was associated with gender $(\mathrm{OR}=0.28 ; \mathrm{CI}=0.44-0.88)$ and with stenosis/occlusion of the right and left medial cerebral artery $(\mathrm{OR}=3.89 ; \mathrm{CI}=1.23-12.33$ and $\mathrm{OR}=6.05 ; \mathrm{CI}=1.34-27.25$, respectively). ALE was associated with age $(\mathrm{OR}=1.77 ; \mathrm{CI}=1.19-2.62)$. Tandem intra- and extracranial vascular stenosis/occlusion was associated significantly $(\mathrm{p}<0.001)$ with territorial infarction but not with the other cerebral lesions.

\section{CONCLUSIONS}

Associations between significant extra-/intracranial steno-occlusive disease and cerebrovascular lesions indicate the importance of panoramic evaluation during pre-surgical work-up. Complete evaluation of extra-/intracranial vasculature and brain can be achieved using minimally invasive MRI as a "one-stop-shop" diagnostic procedure.

\section{MA04:4:3}

\section{IS MR REALLY ESSENTIAL IN THE DIAGNOSIS OF ACUTE CAROTID AND VERTEBRAL DISSECTIONS?}

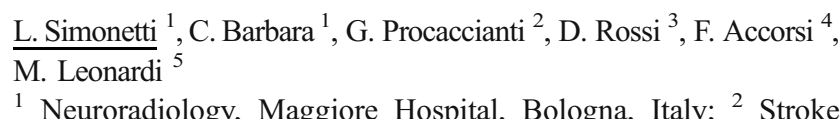
Unit, Maggiore Hospital, Bologna, Italy; ${ }^{3}$ Neurosurgery, Maggiore Hospital, Bologna, Italy; ${ }^{4}$ Ultrasound Diagnostics, Maggiore Hospital, Bologna, Italy; ${ }^{5}$ Neuroradiology, Bellaria Hospital, Bologna, Italy

\section{BACKGROUND}

Vertebral and carotid artery dissections are a relatively common cause of both apparently spontaneous and traumatic stroke in young patients. The literature generally indicates MR as the first choice examination for diagnosis. However, access to MRI in an emergency setting is often hampered by the fact that patients are critically ill and MR facilities are commonly overloaded.

We report our experience in the diagnosis of acute vertebral and carotid artery dissections using CT angiography flanked by echodoppler findings (US).

\section{PATIENTS AND METHODS}

Over a period of two years we examined 15 cases of carotid artery dissection, ten related to trauma and five apparently spontaneous. Patients' ages ranged from five to 46 years. All patients underwent US examination and CT angiography of the neck vessels and intracranial circulation using a multibank 40 slice system with synchronized injection of $50 \mathrm{ml}$ water soluble contrast medium (iopamidol) containing $370 \mathrm{mg} / \mathrm{ml}$ iodine at a flow rate of $4 \mathrm{ml}$ a second.

\section{RESULTS AND CONCLUSIONS}

Combined use of US and CT angiography led to a correct diagnosis of vertebral and carotid artery dissections in all patients. The site of the dissection was always displayed along with any vessel occlusion and its degree of stenosis, free intimal flaps or pseudoaneurysms, the intracranial vasculature and the anatomical features of compensatory circulation. In addition short-term follow-up was monitored by US and the final outcome by CT angiography.

We do not deem MR essential for accurate full diagnosis of spontaneous or traumatic vertebral and carotid artery dissections. 


\section{MA04:4:4}

\section{BASIPARALLEL ANATOMIC SCANNING (BPAS) \\ IN SCREENING FOR VERTEBROBASILAR ARTERY DISSECTION}

\author{
A. Oda, T. Hosoya, Y. Sugai, T. Honma, \\ M. Kanoto \\ Yamagata University,Faculty of Medicine, Yamagata, Japan
}

\section{BACKGROUND AND PURPOSE}

Possibility of new diagnostic finding of vertebrobasilar artery dissection concerning the outer contour of the vessel, which is demonstrated by heavily T2WI, has been reported. This study was performed to evaluate the basiparallel anatomical scanning (BPAS) as a screening method for vertebrobasilar artery dissection.

MATERIAL AND METHOD

We obtained both BPAS and MR angiography (MRA) from 478 consecutive patients who underwent brain MRI in our hospital between February 1 and October 30, 2006. Two observers evaluated combination findings of both BPAS and MRA.

RESULT

Forty-three patients showed external dilatation in BPAS and stenoocclusive change in MRA (type 1), 18 showed external dilatation in BPAS and internal dilatation in MRA (type 2), none showed external dilatation in BPAS without abnormal findings in MRA (type 3), and 30 showed only steno-occlusive change in MRA (type 4). Among 478 patients, vertebrobasilar artery dissection was found in 16 patients; including 12 of 43 patients as type 1, 3 of 18 patients as type 2 , and 1 of 30 patients as type 4 . In 61 patients with external dilatation, 15 showed vertebrobasilar artery dissection. Only a patient with dissection did not show external dilatation.

\section{CONCLUSION}

External dilatation of the vertebral or basilar artery, which is easily observed by BPAS, is an important finding of intracranial arterial dissection. Thus, BPAS with MRA is probably useful as a screening method for vertebrobasilar artery dissection.

\section{MA04:4:5}

3D BASILAR WALL EXTRACTION (3D-BWEX) METHOD: A NEW MRI TECHNIQUE TO VISUALIZE THE INTRACRANIAL VERTEBROBASILAR

ARTERIAL WALL

M. Nagahata ${ }^{1}$, Y. Abe ${ }^{1}$, S. Ono ${ }^{1}$, H. Miura ${ }^{1}$, T. Ohata ${ }^{1}, \mathrm{~F}$. Tsushima ${ }^{1}$, K. Morimoto ${ }^{1}$, H. Seino ${ }^{1}$, T. Tsuji ${ }^{1}$, Y. Kaibara ${ }^{2}$, M. Nakajima ${ }^{2}$

1 Department of Radiology, Hirosaki University School of Medicine, Hirosaki, Japan; ${ }^{2}$ GE Yokogawa Medical Systems, LTD., Tokyo, Japan

\section{PURPOSE}

Basiparallel anatomic scanning (BPAS)-MR imaging is a simple and useful technique to reveal the vascular outer contour of the intracranial vertebrobasilar artery. We can imagine the vascular wall thickness by comparing a BPAS-MR image with a three dimensional (3D) time-of flight (TOF) MRA. BPAS-MR image or MRA, but we cannot directly visualize the vascular wall. We report a new 3D MRI technique to visualize the intracranial arterial wall.

\section{METHODS}

Using a 1.5-T MR machine, we obtained a coronal 3D TOF images and also the coronal 3D heavily $\mathrm{T} 2$ weighted images with same section thickness and same slab. Summated acquisition time of both pulse sequences was about 8 minutes. Then we superimposed coronal TOF images onto the heavily T2 weighted images on a MR operating console. These post-processed 3D MR images are able to be reformatted multi-planarly on a work station and to be observed by cine mode. Gray-scale reversal is helpful to see the vascular wall. We call this imaging and post-processing technique as 3D basilar wall extraction (3D-BWEX) method.

\section{RESULTS}

Since September 2005, we also performed 3D-BWEX method with routine brain MR examination in 43 patients who were suspected of posterior cranial fossa lesions. The vascular wall of the entire intracranial vertebrobasilar artery was clearly shown in 33 cases by the 3D-BWEX method. In 7 cases, the basilar arterial trunk was obscured by the magnetizing susceptibility artifacts due to air in the sphenoid sinus. Superimposition of 3D images had failed due to patients' motion during the MR acquisition in 3 cases.

CONCLUSION

3D-BWEX method is a clinically applicable MR technique to visualize the vascular wall of intracranial vertebrobasilar artery. Although the vascular wall depicted by this imaging technique does not mean a true thickness, this method would be helpful to recognize vascular wall lesions of the vertebrobasilar system.

\section{MA04:4:6}

IT IS POSSIBLE TO STUDY ULCERATED CAROTID PLAQUE BY USING MULTIDETECTOR-ROW CT ?

L. Saba ${ }^{1}$, G. Mallarini ${ }^{1}$

Policlinico Universitario di Monserrato, Cagliari, Italy

\section{PURPOSE}

Ulcerated carotid plaque is an important risk factor for cerebrovascular events, as clearly demonstrated in previous studies and its presence may change the risk class of a patient by moving himher from medical therapy to surgical carotid endarterectomy or percutaneous angioplasty. Aim of this work is to describe multidetector-row CT Angiography imaging features of ulcerated carotid plaque.

\section{MATERIAL AND METHODS}

MDCTA images of 37 patients (29 males; 8 females; mean age 70 years, age range 57-81 years) with carotid ulcerated plaque surgically confirmed by CEA were reviewed. MDCTA exams were 
performed with a multi-detector row scanner, after intravenous bolus administration of $130 \mathrm{~mL}$ of non-ionic contrast material using a 3$5 \mathrm{~mL} / \mathrm{sec}$ flow rate. We assessed each patient by using axial scans, multiplanar reconstruction (MPR), maximum intensity projection (MIP), single surface display (SSD) and volume rendering (VR) techniques. In some patients we performed an histological analysis. RESULTS

We observed that plaque ulcerations increased with severity of stenosis and soft plaques showed this lesion more easily (2437). Morphology of ulcerated carotid plaque is widely variable but we observed in particular crateriform morphology. 3-D images and specially VR show an high efficacy in the characterization of plaques ulceration.

\section{CONCLUSION}

Carotid plaque ulceration is an important plaque complication and to know its different aspect in CTA is important to correctly detect it. Fatty plaque and high grade stenosis are the most frequent localization for ulcerated plaque and usually it is present a crateriform morphology. Sometimes differential diagnosis may be not simple in some type of dissections and parietal calcifications may produce artefacts.

\section{MA04:4:7}

\section{ACCURACY \& INTEROBSERVER RELIABILITY OF NON-RIGID REGISTRATION CT ANGIOGRAPHY IN THE EVALUATION OF CAROTID STENOSIS}

P. Vanacker $^{1}, \mathrm{~S}$. Heye ${ }^{2}$, D. Loeckx ${ }^{3}$, G. Wilms ${ }^{2}$, V. Thijs ${ }^{1}$

${ }^{1}$ Department of Neurology, Catholic University Leuven, Belgium;

2 Department of Neuroradiology, Catholic University Leuven, Belgium; ${ }^{3}$ Medical Image Computing, Catholic University Leuven, Belgium

\section{PURPOSE}

A novel non-rigid registration algorithm is able to reduce noise levels and better visualize the vascular lumen by reducing the intensity of hyperdense, non-vascular structures on computed tomography angiography (3D MASK). We analysed the diagnostic accuracy of 3D MASK in the quantitative evaluation of moderate (50-69\%) and high-grade carotid artery stenosis (70-99\%).

\section{METHODS}

We compared the accuracy of detecting a $>50 \%$ and $>70 \%$ stenosis of the internal carotid artery with 3D MASK CTA versus the combination of carotid ultrasound and contrast enhanced MRA. Three observers independently analyzed carotids with 3D MASK CTA and contrast enhanced MRA. Images were analysed using NASCET criteria (North Amercian Symptomatic Carotid Endarterectomy Trial). In addition a semi-automatic analysis of the degree of stenosis was done using postprocessing software on 3D MASK images. RESULTS

Sixty eight carotids were analysed. The sensitivity and specificity for detecting stenosis $>50 \%$ was 0.96 (CI 95\% $0.89-0.99$ ) and 0.67 (CI 95\% 0.48-0.76) for 3D MASK CTA compared to ultrasound and CE-MRA. For the detection of high-grade stenosis $(>70 \%)$ an identical sensitivity $(0.96$, CI $95 \% 0.91-99)$ with a lower specificity $(0.46$, CI $95 \% \quad 0.25-0.58)$ was found. The interobserver reliability for the 3D MASK images (Pearson Correlation Coefficient $=0.97$, CI 95\% 0.95-0.98) was higher in comparison with MRA ( $\mathrm{P}=0.89$, CI 95\% 0.82-0.93). The mean differences between the three observers were significantly smaller for 3D MASK CT Angiography than MRA (respectively $4.73 \%$ and $7.48 \%, p=0,049)$. These differences were more pronounced on 3D MASK in the $0-50 \%$ interval whereas on CE-MRA differences were found for all degrees of severity of stenosis.

\section{CONCLUSIONS}

The new postprocessing 3D MASK CTA based on non-rigid registration has high sensitivity, good specificity and limited variation between the observers in comparison with carotid ultrasound and contrast-enhanced MRA. Further studies should compare this technique with intraarterial digital subtraction angiography.

\section{MA04:4:8}

\section{MICROEMBOLIC LESIONS IN DIFFUSION MRI AFTER CAROTID STENTING IN CORRELATION TO STENOSIS DEGREE ASSESSED BY MDCT.}

S. Klein, M. Jeltsch, M. Hoffmann, H. Brambs, A. Aschoff, B. Schmitz University Hospital of Ulm, Ulm, Germany

\section{PURPOSE}

The potential efficacy of filter devices during internal carotid artery stenting is an ongoing matter of controversy and predictive factors which distinguish high risk patients with a high load of cerebral microembolisations are not known.

The current study was undertaken to asses the potential correlation between the incidence of new ischemic lesions after filterprotected stent placement in the internal carotid artery detected by diffusion weighted MR imaging (DWI) and the degree of internal carotid stenosis before and after stent implantation by Multi-detector CT-Angiography.

MATERIAL AND METHODS

Diffusion weigthed MR imaging (1,5 T Symphony, Siemens, Erlangen) was performed within 48 hours before and after filterprotected stent implantation in the internal carotid artery in 30 patients with symptomatic stenosis. Vascular territory, size and number of new diffusion restricted lesions were assessed by two investigators. The preinterventional degree of internal artery stenosis and the in-stent lumen after the intervention was determined by multi-detector CTAngiograhy (Brilliance 40, Philips, Cleveland) using diameter and area based measurements methods. Statistical analysis were performed to assess the correlation between stenosis degree and cerebral microembolization represented by new lesions in diffusion MR imaging. RESULTS

Neuroprotected carotid stent placement was successful in all 30 patients but in two cases transient ischemic attack occurred as a minor procedure-related complication. Overall 9 of 30 patients showed new lesions in DWI but only in two cases more than 3 microembolic lesions were detected. The preinterventional degree of carotid artery 
stenosis showed no correlation (Pearsons correlation coefficient $\mathrm{P}=$ $-0,08)$ to the incidence of new brain lesions in diffusion weighted MRI. The extent of residual in-stent stenosis after stent implantion correlated moderately with the incidence of new lesions in diffusion MRI $(\mathrm{P}=0,59)$.

\section{CONCLUSION}

A high preinterventional degree in carotid artery stenosis represents no risk factor for microembolic lesions after filterprotected stent implantation in the internal carotid artery whereas residual in-stent stenosis seems to be moderately correlated with higher rates of new lesions in diffusion MR imaging.

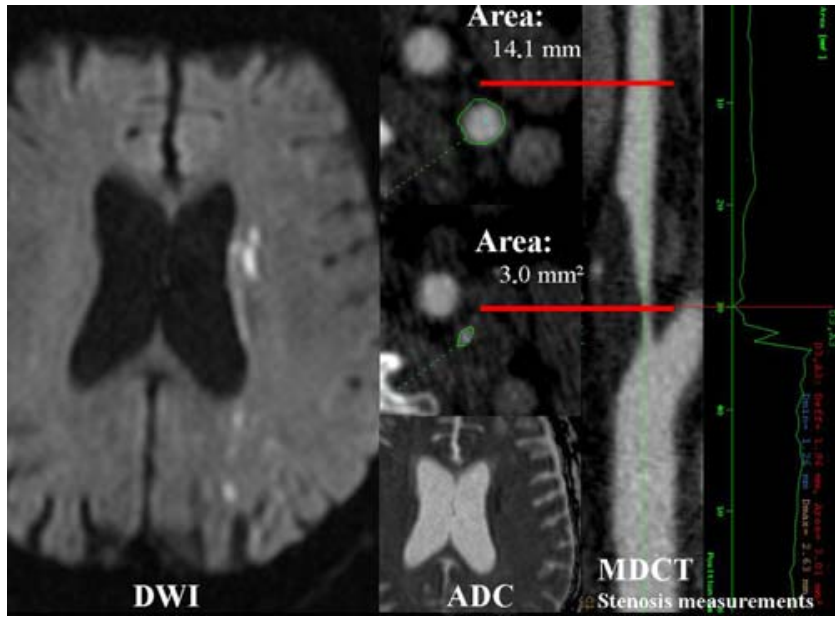

\section{MA04:4:9}

\section{PREVALENCE OF IN-STENT WALL ADHERENT THROMBI} DIRECTLY SEEN AFTER CAROTID ARTERY STENTING DEPICTED BY HIGH-RESOLUTION CT ANGIOGRAPHY

\section{S. Klein, A. Aschoff, R. Huber, B. Schmitz}

University Hospital of Ulm, Ulm, Germany

\section{PURPOSE}

Carotid artery stenting (CAS) is an emerging therapy for carotid artery stenosis. However it is associated with method inherent risk of embolic stroke not only during the interventional procedure but also in the first hours after the intervention. The purpose of the present study was to investigate the prevalence of early in-stent thrombi as potential embolic source.

METHOD AND MATERIALS

CTA was performed in 50 patients within 3 days (median 1 day) after CAS. All patients were pretreated with a combination of 2 antiplatelet agents (Clopidogrel and Aspirin) starting 2 days before the intervention and received iv heparin during and for $24 \mathrm{~h}$ after the intervention. All patients were examined on a 40-channel MDCT (Brilliance 40, Philips, Cleveland, $\mathrm{OH}$ ) using a collimation of $40 \times 0,65 \mathrm{~mm}$ with 0,67 slice thickness, a $0,3 \mathrm{~mm}$ reconstruction interval and a matrix of $768 \times 768$ after bolus injection of $1,1 \mathrm{ml} / \mathrm{kg}$ BW of Imeron 400 (Bracco, Milano, Italy) at a rate of 3,5 ml/sec. Original images and curved, multiplanar Reformations were reviewed by two experienced readers. Wall-adherent structures within the stent lumen equivalent to thrombi were classified by measured size on a dedicated workstation (MxView, Philips).

\section{RESULTS}

CTA detected 7 small and 1 medium sized thrombus within the stent lumen after CAS in 8 out of 50 patients $(16 \%)$. The patient with the medium sized thrombus experienced a symptomatic stroke. CONCLUSION

High resolution CTA is able to depict early in-stent thrombi after carotid artery stenting which are associated which a certain risk of embolic stroke. Thrombi may be found frequently despite a combination of antiplatelet therapy and iv heparin. More aggressive antiplatelet therapy and screening for aspiring resistances might be indicated in this subgroup of patients after CAS.

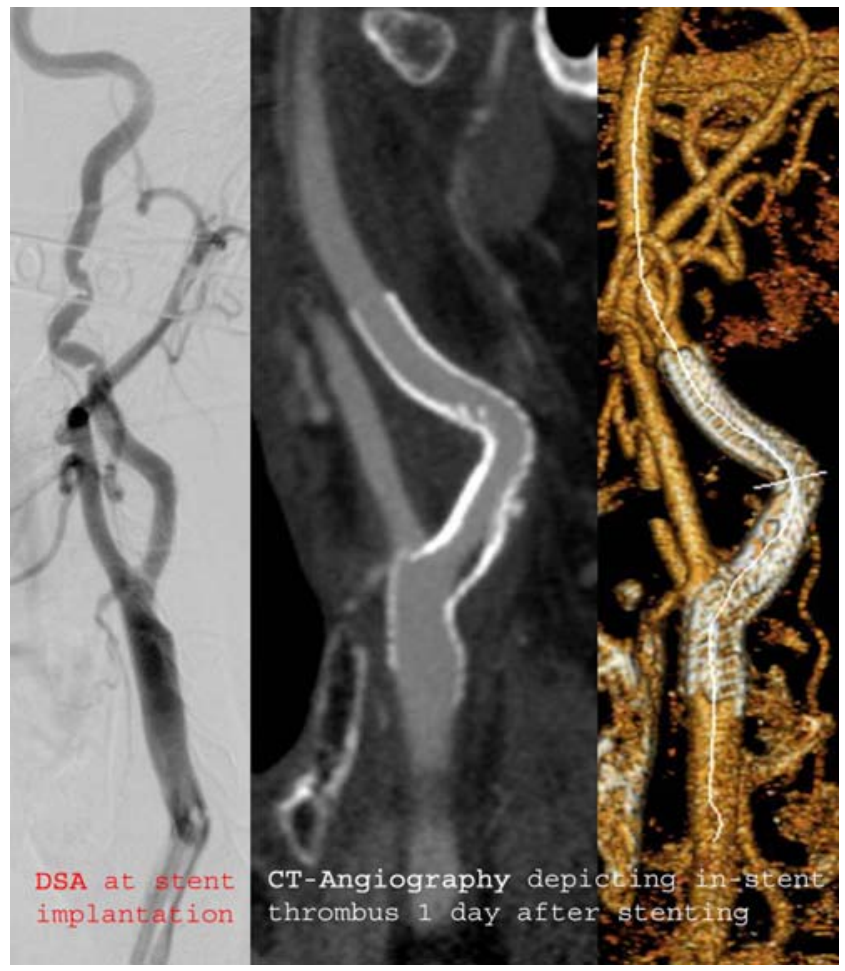


Scirocco - Libeccio Room

\section{SL01}

09.50-11.15 - Stroke \& tumors

\section{SL01:5:1}

PREDICTIVE VALUE OF VISUALLY ASSESSED PERFUSION LESIONS IN 100 STROKE PATIENTS

S. Siemonsen, T. Fitting, G. Thomalla, A. Kruetzelmann, J. Fiehler

University Hospital Hamburg-Eppendorf, Hamburg, Germany

\section{BACKGROUND}

Few stroke MR studies have evaluated the value of visual assessment of perfusion lesions which is crucial for routine application. We sought to identify the most relevant perfusion parameter in a large patient group.

METHODS

Hundred acute stroke patients were treated with tPA. MRI was performed within 6 hours of symptom onset and after 1-11 days. MRI protocol included diffusion-weighted (DWI/ADC=apparent diffusion coefficient), fluid-attenuated-inversion-recovery (FLAIR), perfusion imaging (PI). Maps for different perfusion parameters, i.e. cerebral blood volume (CBV), cerebral blood flow (CBF) and time to peak (TTP) were calculated. Lesion sizes of all perfusion parameters were visually compared to corresponding ADC and final lesion size.

RESULTS

The final infarct size was overestimated by TTP (92\%), and CBF (73\%) lesions. The ADC and CBV lesions were rated smaller than the final infarct lesion in 52\%.OR for infarct growth was $2.22(95 \%$ CI $0.15-63.25 ; \mathrm{p}=0.511)$ for $\mathrm{TTP}>\mathrm{ADC}$ and 2.47 (95\% CI 0.70 $9.20 ; \mathrm{p}=0.117$ ) for $\mathrm{CBF}>\mathrm{ADC}$. The only significantly increased $\mathrm{OR}$ of 3.97 (95\% CI 1.58-10.14; $\mathrm{p}=0.001)$ for predicting lesion growth was observed for $\mathrm{CBV}>\mathrm{ADC}$ with a sensitivity of $0.60(95 \% \mathrm{CI}$ $0.45-0.73$ ), a specificity of 0.73 (95\% CI $0.58-0.85)$, positive predictive value (PPV) was 0.70 (95\% CI $0.55-0.83)$, negative predictive value (NPV) was 0.63 (95\% CI 0.49-0.75).

DISCUSSION

Of all tested mismatch patterns the $\mathrm{CBV}>\mathrm{ADC}$ mismatch was found to be the strongest predictor of lesion growth. Visual assessment of other parameters like TTP and CBF generally resulted in an overestimation of infarct sizes and the presence of a TTP $>$ ADC or $\mathrm{CBF}>\mathrm{ADC}$ mismatch was not significantly asscociated with lesion growth.

\section{SL01:5:2}

\section{DETERMINING THE EXTENT OF THE INFARCT CORE WITH VISUAL ASSESSMENT OF PCT CBV COLOR-MAPS. COMPARISON WITH DELAYED DIFFUSION-WEIGHTED IMAGES IN PATIENTS WITH RECANALIZATION}

A. Cianfoni $^{1}{ }^{1}$, M. Basile ${ }^{1}$, F. Piludu ${ }^{1}$, $\bar{C}_{\text {. Schiarelli }}{ }^{1}$, C. Falcone ${ }^{1}$, F. Pilato ${ }^{2}$, V. Di Lazzaro ${ }^{2}$, C. Colosimo ${ }^{1}$

${ }^{1}$ Dept. Bio-Images Radiological Sciences - Catholic University Policlinico Gemelli, Rome, Italy; ${ }^{2}$ Dept. Neurology - Catholic University - Policlinico Gemelli, Rome, Italy

Perfusion-CT (PCT) has proved to be a valid technique to image ischemic penumbra in acute stroke patients. Quantitative cerebral blood volume (CBV) measurements differentiate infarcted from ischemic tissue. Nevertheless quantitative CBV assessment relies on complex mathematical processes, sophisticated softwares, often not commercially available, and might be influenced by physiological and technical factors. We sought to determine whether exclusively visual assessment of PCT-CBV color-maps recognizes the infarct-core, within the hypoperfused area.

In a series of acute stroke patients underwent PCT study at admission, we considered a sub-group of 12 consecutive patients, with intra-cranial arterial occlusion at admission CT-angiography (CTA), and evidence of recanalization at MRI and MR-angiography (MRA) performed 24-48 h later. In 6/12 patients i-v rTPA thrombolysis was administered. MRI included oblique-axial DWI sequence, oriented like PCT, and 3D-TOF-MRA sequence. PCT data were post-processed with a commercially available software. Color-maps were visually evaluated with flexible window setting. Areas of visually-assessed reduced CBV, and of high signal in the corresponding sections of the DWI images were drawn. The sum of areas with reduced CBV was compared with the sum of areas with DWI abnormality. Linear regression analysis compared infarct size on the corresponding PCT and DWI sections.

The extent of reduced CBV on admission PCT and of restricted diffusion on delayed DWI MR images significantly correlated $(\mathrm{p}<$ $0.05 ; \mathrm{r} 2=0.822$ ). There was a trend toward larger areas of infarct visible on DWI compared to the areas of reduced CBV on PCT maps, with high specificity of PCT-CBV maps. In 1 patient with lacunar infarct the PCT map failed to detect a CBV defect Areas of visually assessed reduced PCT-CBV correlate with areas of delayed DWI abnormality in stroke patients with arterial recanalization

Visual assessment of reduced CBV on PCT color-maps seems a valid approach to define the extent of the infarct core in acute stroke patients 


\section{SL01:5:3}

PERMEABILITY MAPS IN ACUTE ISCHEMIC STROKE USING DYNAMIC SUSCEPTIBILITY

CONTRAST MR IMAGING: PREDICTING HEMORRHAGIC TRANSFORMATION

$\underline{\text { C. Ryu }}^{1}{ }^{1}$, G. Jahng ${ }^{1}$, S. Kim ${ }^{3}$, D. Yang ${ }^{1}$, W. Jin ${ }^{1}$, H. Kim ${ }^{1}$, D. $\overline{\text { Nam }}^{1}$, J. Ryu ${ }^{1}$, W. Choi ${ }^{2}$

${ }^{1}$ EWNMC, colleage of medicine, Kyung-Hee university, Seoul, South Korea; ${ }^{2}$ Kyung-Hee univeristy medical center, colleage of medicine, Kyung-Hee university, Seoul, South Korea; ${ }^{3}$ Asan medical center, colleage of medicine, Ulsan university, Seoul, South Korea

Hemorrhagic transformation is a serious complication in acute ischemic stroke, particularly in those patients who are treated with thrombolysis. The purpose of this study was to determine whether elevated vascular permeability measured from dynamic susceptibility contrast MR imaging (DSC-MRI)) is associated with subsequent hemorrhagic transformation (HT) in patients with acute ischemic stroke.

21 patients (M:F=13:8, mean age: 65.5 years) with acute ischemic stroke (HT group) were retrospectively evaluated. Inclusion criteria are below: 1) MR diffusion-weighted imaging and perfusion imaging (DSC-MRI) within 12 hours after onset, 2) MCA territorial infarction, and 3) large HT within 7 days. As the presence of mass effect of HT, patients were classified as large HT and small HT group. 17 patients (M:F=6:11, mean age: 69.9 years) with acute MCA territorial infarction, which had no evidence of HT, were randomly selected as control group. For evaluating permeability of ischemic lesion, the parameter map of permeability was calculated from perfusion MRI, along with $\mathrm{CBV}$, MTT, and CBF map. Mean permeability values (Ktans) were recorded from ROI in abnormality (HT group: HT on follow-up image, control group: infarct core on DWI) and contralateral normal brain. The Ktrans were compared between three groups (large HT, small HT and control group) by using onway-ANOVA.

In all cases, the permeability map was successfully processed from DSC MRI, along with other perfusion parameter maps (CBV, MTT, and CBF). Large HT group was 11 and small HT was 10. The Ktrans was statistically different between large HT group and the other groups (between groups: $p=0.0219$; Large HT-small HT: mean difference $0.015 \mathrm{p}<0.05$; Large HT- control: mean difference 0.012, $\mathrm{p}<0.05$; Small HT-control: mean difference $0.003, \mathrm{p}>0.05$ )

The permeability parameter, as measured on DSC-MRI, would be helpful for predicting hemorrhagic transformation in patients with acute ischemic stroke.

\section{SL01:5:4}

\section{BLOOD OXYGEN LEVEL DEPENDEND (BOLD)-LESIONS IN MRI PREDICT INFARCT GROWTH IN ACUTE STROKE PATIENTS}

S. Siemonsen, T. Fitting, G. Thomalla,

A. Kruetzelmann, J. Fiehler

University Hospital Hamburg-Eppendorf, Hamburg, Germany

\section{BACKGROUND}

Hypointense lesions in BOLD-imaging correspond to increased deoxy- $\mathrm{Hb}$ concentration and may indicate the increased oxygen extraction fraction (OEF) in acute ischemic stroke. We hypothesized that $\mathrm{T} 2$ '-lesions represent the penumbra and when exceeding the ADC lesion are predictor of infarct growth.

\section{METHODS}

Hundred acute stroke patients were treated with tPA. MRI was performed within the first 6 hours of symptom onset and after 1-11 days. MRI protocol included diffusion-weighted (DWI/ $\mathrm{ADC}=$ apparent diffusion coefficient) and quantitative $\mathrm{T} 2$ and T2* imaging (qT2, qT2*). BOLD images (T2') with total acquisition time of 1:20 minutes were calculated using $1 / \mathrm{T}^{\prime}=$ $1 / \mathrm{T} 2 *-1 / \mathrm{T} 2$. T2' lesions were visually compared to $\mathrm{ADC}$ lesions.

RESULTS

Sensitivity of T2'>ADC mismatch for later infarct growth was 0.87 (95\% CI $0.74-0.94)$, specificity was 0.42 (95\% CI 0.28 $0.57)$, positive predictive value (PPV) was $0.62(95 \%$ CI 0.50 $0.73)$, and negative predictive value (NPV) was $0.74(95 \% \mathrm{CI}$ 0.54-0.89). The OR for infarct growth in the presence of a T2'> ADC mismatch was 4.59 (95\% CI 1.57-13.85; $\mathrm{p}=0.002)$.

\section{DISCUSSION}

T2'-BOLD technique was applicable in routine MRI of acute stroke. T2' lesions exceeded the lesion in diffusion weighted imaging in most of the stroke patients and were associated with later infarct growth. This predictive value for infarct evolution may be worth considering in treatment decisions in the future. A prospective multicentre study is under way.

\section{SL01:5:5}

\section{NEURORADIOLOGICAL TOOLS IN DETECTION ISOLATED CORTICAL VENOUS THROMBOSIS (CVT)}

C. Cinnante, A. Costa, C. Sina, S. Avignone, M. Isalberti, N. Nuzzi, P. Machì, V. Branca

Neuroradiologia Diagnostica ed Interventistica Fondazione Ospedale Maggiore Policlinico Mangiagalli Regina Elena IRCCS Milano, Italy

\section{PURPOSE}

cortical venous thrombosis (CVT), without concomitant dural sinus thrombosis, is an uncommon disorder and the clinical diagnosis is difficult due to a wide spectrum of clinical manifes- 
tations. Therefore the diagnosis is typically based on imaging studies. Aim of this study is to assess the best neuroradiological diagnostic tool to detect this disease.

\section{METHODS}

From January 2005 to April 2007 we evaluated seven patients (five men and two women, mean age 73) who presented in our Emergency Room with neurological symptoms mainly represented by sensorimotor strokes. In all of them noncontast CT scan documented a focal SAH mainly localized in central and precentral regions. MR and MR 2D-TOF venography examinations of the brain was subsequently obtained within 24 hours of the initial symptoms. The MR exam was conducted with SE T1 weighted images in the three orthogonal planes, dual echo T2 weighted axial, and coronal FLAIR images, completed with Diffusion weighted images.

RESULTS

all patients had FLAIR imaging findings that demostrated localized, unilateral, abnormal high signal intensity within the sulci, corresponding to the subarachnoid hemorrhage at the CT scan. The parenchyma appear normal, even in DWI images, and MR venography demonstrated patency of the major dural sinuses. $\mathrm{T} 1$ and proton density weighted images, instead, showed a tubular structures of high signal in the region adjacent of SAH, suggesting a diagnosis of isolated cortical venous thrombosis.

\section{CONCLUSIONS}

MR imaging appears as the best neuroradiological tool to assess CVT, because is able to detect the clot within the thrombosed cortical vein, and the possible parenchymal changes associated with the thrombosis. Conventional angiography, still the gold standard as diagnostic examination for dural venous thrombosis, is on the contrary particularly difficult in CVT diagnosis, because the pattern of cortical venous drainage is variable and often asymmetric. MR imaging is also far more sensitive than CT, avoiding intravenous contrast media administration and ionizing radiation.

\section{SL01:5:6}

\section{NEUROSURGICAL PLANNING WITH DTI: CLINICAL RELEVANCE IN PATIENTS WITH BRAIN NEOPLASMS}

\author{
A. Romano ${ }^{1}$, M. Ferrante ${ }^{1}$, F. Fasoli ${ }^{1}$, \\ L. Ferrante $^{2}$, G. D'andrea ${ }^{2}$, V. Cipriani ${ }^{1}$, \\ L. Fantozzi ${ }^{1}$, A. Bozzao ${ }^{1}$ \\ 1 Department of Neuroradiology, S.Andrea Hospital University
} Sapienza, Rome, Italy; ${ }^{2}$ Department of Neurosurgery, S.Andrea Hospital University Sapienza, Rome, Italy

\section{PURPOSE}

To evaluate the impact of Diffusion Tensor Imaging (DTI) on the neuro- surgical approach in patients with cerebral tumors, identifying white matter tracts close to the neoplasm.

MATERIALS AND METHODS

DTI was performed in 25 patients undergoing surgery for cerebral tumors (11 glioblastomas, 6 metastasis, 3 high grade gliomas, 4 low grade gliomas, 1 lymphoma). Cortico-spinal tracts, optic radiations and fasciculus arcuatus were evaluated in threedimensional and considered for neurosurgical planning with neuronavigation. Two neurosurgeons were asked whether surgical approach was changed on the basis of DTI and in which and how many cases DTI changed the limits of surgical resection. A control DTI study was performed after one month from surgery. Clinical symptoms were considered as well before and after surgery.

\section{RESULTS}

Only in one patient no change in the surgical procedure was observed.

40 of the 75 white matter (WM) tracts considered did not show significant relationship with the tumor, evidence of dislocation or were not completely visualized due to the anisotropy induced by the tumor $(23 \%$ of all patients and $30 \%$ of those located close to the neoplasm). Considering the remaining $35 \mathrm{WM}$ tracts, the surgical approach (corticotomy) was modified a priori by the analysis of DTI in 4 patients $(16 \%)$ and allowed useful information concerning the limits of surgical resection in 17 patients (68\%). Overall there was an impact of DTI on the surgical procedures in $80 \%$ of cases.

17 of 25 patients showed clinical deficits correlated to an involvement of the considered WM tracts. After surgery one patient 1 showed a new deficit (aphasia), none had worsening of symptoms and in 14 of 17 symptoms were getting better. CONCLUSION

DTI can really help neurosurgeon in preserving major fiber tracts during the surgical procedure.

\section{SL01:5:7}

\section{UNILOCULAR OR MULTILOCULAR RECURRENT GLIOBLASTOMA MULTIFORME: ANALYSIS OF PREOPERATIVE MRI DATA}

\author{
A. Bink $^{1}$, T. Meinel ${ }^{1}$, H. Ackermann ${ }^{2}$, \\ $\overline{\text { F. Zanella }}^{1}$, H. Lanfermann ${ }^{1}$ \\ ${ }^{1}$ Department of Neuroradiology, Frankfurt/Main, Germany; \\ ${ }^{2}$ Department of Biomathmatics, Frankfurt/Main, Germany
}

\section{PURPOSE}

To analyze MRI data of patients with unilocular or multilocular recurrences after total gross resection of primary glioblastoma multiforme (GBM) in order to evaluate preoperative findings on MRI that may influence if a recurrence develops uni- or multilocular.

\section{METHODS}

93 patients, i.e. 73 patients with unilocular and 20 patients with multilocular recurrences of GBM, were included (39 f, $54 \mathrm{~m}$; mean age: 58 years). The following parameter were documented: patient's age, location of primary GBM, midline shift, involvement of eloquent areas, contact to ventricles, size of edema, tumor volume and time to progression. Cross tables, Wilcoxon-MannWhitney-test and logistic regression analysis were used as statistical methods. 


\section{RESULTS}

No significant differences between patients with uni- or multilocular recurrences were found for these preoperative MRI data: location of primary GBM, midline shift, involvement of eloquent areas and contact to ventricles.

The median values of age showed no differences between both patient groups whereas the median values for time to progression, size of edema and tumor volume were different. Logistic regression revealed a significant relation for tumor volume $(\mathrm{p}<0.044)$ : Larger primary GBM had a higher probability of a multilocular recurrence. Size of edema had no significant influence $(p<0.078)$ neither had time to progression $(\mathrm{p}<0.189)$ influence on uni- or multilocularity. CONCLUSIONS

A significant relationship between tumor volume and uni- or multilocular manifestation of recurrent GBM was found. Whereas other parameters, e.g. contact to ventricles, had no impact on unior multilocularity.

\section{SL01:5:8}

\section{INALIENABILITY OF POSTOPERATIVE MR IMAGING AFTER NEUROSURGICAL RESECTION OF MALIGNANT GLIOMAS.}

$\underline{\text { S. Ulmer }}{ }^{1}$, O. Jansen ${ }^{1}$, F. Barker ${ }^{2}$, J. Henson ${ }^{3}$

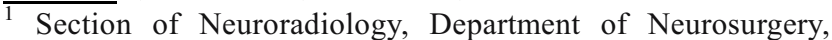
University Hospital of Schleswig-Holstein, Kiel, Germany;

${ }^{2}$ Neurosurgical Service, Massachusetts General Hospital, Boston, USA; ${ }^{3}$ Pappas Center for Neuro-Oncology, Massachusetts General Hospital, Boston, USA

\section{PURPOSE}

It is understood that some kind of imaging modality (CT or MRI) is performed within 72 hours following a neurosurgical resection of a brain tumor. CT is considered adequate as it may rule out space-occupying bleedings in the resection cavity or major strokes. In some cases new neurological symptoms occur after the resection of a tumor without corresponding imaging findings on $\mathrm{CCT}$.

\section{MATERIALS AND METHODS}

We analyzed the data of 50 consecutive patients having received an immediate postoperative MRI after the resection of a glioblastoma multiforme ( $<72$ hours) including a diffusion weighted sequence (DWI), T1 +/- Gd and T2-weighted sequences and a follow-up exam within 90 days. Areas of restricted diffusion were measured. Clinical noted and charats were reviewed.

RESULTS

In 35 of the 50 patients $(70 \%)$ we found areas of restricted diffusion as a rounded focus extending from the margin of the cavity into adjacent brain tissue, often along the expected distribution of transcortical medullary arteries. In 32 of these 35 cases $(91 \%)$ the area of restricted diffusion had developped into a cystic encephalomalacia on follow-up T2-weighted images. Enhancement was present at the area of encephalomalacia in $15 / 35(43 \%)$ infarcts. Ten patients had new neurologic deficits postoperatively. In six of these cases the deficits could be explained by the region of postoperative diffusion abnormality. CONCLUSION

Areas of restricted diffusion are frequent following neurosurgical resection of glioma. Without a DWI sequence minor lesions might be missed that can present as a encephalomalacia with or without enhancement in follow-up MRI. Without knowledge of these lesions new enhancing foci could be misinterpreted as recurrence of the tumor.

\section{SL01:5:9}

\section{THE ROLE OF ADVANCED MRI TECHIQUES IN ASSESSING LOW-GRADE GLIOMAS: PRELIMINARY RESULTS}

A. Erbetta ${ }^{1}$, F. Ghielmetti ${ }^{1}$, M. Eoli ${ }^{2}$,

$\overline{\text { M. Grisoli }}^{1}$, E. Ciceri ${ }^{1}$, M. Farinotti ${ }^{2}$, C. Falcone ${ }^{2}$,

B. Pollo ${ }^{3}$, G. Broggi ${ }^{4}$, A. Boiardi ${ }^{2}$, G. Finocchiaro ${ }^{5}$, G. Filippini $^{2}$, M. Bruzzone ${ }^{1}$

${ }^{1}$ Dept. of Neuroradiology, IRCCS NEUROLOGICO C. BESTA, Milano, Italy; ${ }^{2}$ Dept. of Neurology, IRCCS NEUROLOGICO C. BESTA, Milano, Italy; ${ }^{3}$ Dept. of Neuropathology ,IRCCS NEUROLOGICO C. BESTA, MILANO Milano, Italy; ${ }^{4}$ Dept. of Neurosurgery, IRCCS NEUROLOGICO C. BESTA, Milano, Italy; ${ }^{5}$ Dept. Experimental Neuro-Oncology, IRCCS NEUROLOGICO C. BESTA, Milano, Italy

\section{PURPOSE}

Prospective radiological grading of primary cerebral gliomas can provide relevant informations for clinical management. Sensitivity in defining the grade of Magnetic Resonance Imaging (MRI) is about $60-80 \%$ in defining grading. To assess the accuracy of Perfusion (PWI), Diffusion (DWI) and Proton-Spectroscopy, we collected all patients with MRI suggesting glioma in absence of necrosis, relevant oedema, and enhancing volume $<30 \%$ total tumor volume, from December 2006 to April 2007. In 10 patients the preoperative diagnosis was low grade glioma.

\section{METHODS}

Soon before surgery, 16 patients ( $11 \mathrm{~m}, .5 \mathrm{f}$,. age range: $26-77)$ were investigated by $1.5 \mathrm{~T}$ MRI with a protocol including DWI, PWI and proton spectroscopy. ROI analyses were performed on ADC and rCBV maps on the different appearing at $\mathrm{T} 2$ imaging portions of the tumor and on the contralateral normal region. After surgery histopathological analysis confirmed the diagnosis of low-grade glioma in 7 patients ( 2 fibrillary astrocytoma, 1 oligodendroglioma, 4 oligoastrocytomas). Other patients had a grade III (6 pts.) and grade IV (3 pts.) gliomas

\section{RESULTS}

Oligoastrocytomas grade II have ADC values ranging between 12.5 and $\mathrm{CBV}$ values ranging between $0.4-1.2$ with respect to contralateral normal values. Oligodendroglioma is characterized by high value of rCBV. Fibrillary astrocytomas have high ADC value and the lowest rCBV values. Secondary GBM show similar values of $\mathrm{ADC}$ and $\mathrm{rCBV}$ as grade II, while de novo GBM has high $\mathrm{rCBV}$ (1.5 times respect to contralateral) e low ADC (0.3). 
Excluding oligodendroglioma in all the 6 patients with confirmed low-grade tumors the $\mathrm{Cho} / \mathrm{CR}$ ratio was $<1.8$. These preliminary results confirm the diagnostic role of advanced MRI in association with standard imaging. The limitation is differentiation between grade II and III tumors. Threshold values and ROI positioning into the lesion and in peritumoral tissue is of critical relevance.

\section{SL01:5:10}

\section{HIGH B-VALUE DIFFUSION PROPERTIES IN HIGH GRADE GLIOMAS}

A. Elias ${ }^{1}$, P. Sundgren ${ }^{1}$, B. Ross ${ }^{1}$,

M. Ivancevic ${ }^{1}$, C. Tsien ${ }^{2}$, L. Junck ${ }^{3}$,

T. Chenevert ${ }^{1}$

${ }^{1}$ Department of Radiology, University of Michigan, Ann Arbor, USA; ${ }^{2}$ Department of Radiation Oncology, University of Michigan, Ann Arbor, USA; ${ }^{3}$ Department of Neurology, University of Michigan, Ann Arbor, USA

\section{PURPOSE}

To investigate multiexponential diffusion features in normal tissues and high grade glioma in patients by applying a clinically-practical high b-value DWI protocol with the objective to provide baseline values of landmark tissues and tumor over a wide range of $b$-values as prerequisites to therapy response studies. MATERIALS AND METHODS

Pre-therapy baseline imaging included anatomic, DWI, and PWI performed on a $3 \mathrm{~T}$ system (Philips Achieva) were performed in 13 high grade glioma patients (10 GBM; 1 G3 anaplastic glioma; 1 anaplastic oligo; 1 G3 astrocytoma; 9male, 4 female; mean age 53, range 20-79). ). DWI consisted of 3-axis isotropic weighting at diffusion sensitivities of $b=0,1000,2000,4000 \mathrm{~mm} 2 / \mathrm{sec}$ using SSEPI $(\mathrm{TR} / \mathrm{TE}=8700 / 60 \mathrm{~ms})$ and parallel imaging $(\mathrm{SENSE}=3)$. Anatomic, DWI, perfusion and derivative images were spatially coregistered, and $\mathrm{ADC}$ values in different brain regions were calculated from image pairs: $\mathrm{b}=0$ \& 1000 (ADC1k, i.e. ¡§conventional;" ADC); $\mathrm{b}=1000 \& 2000$ (ADC2k); and $\mathrm{b}=2000$ $\& 4000(\mathrm{ADC} 4 \mathrm{k})$ and the ratio $\mathrm{SADCR}^{*}{ }^{*}, \mathrm{k}\{\mathrm{ADC} 1 \mathrm{k}\} /$ $\{\mathrm{ADC} 4 \mathrm{k}\}$.

\section{RESULTS}

While ADC1k, ADC2k, ADC4k images were windowed independently to better show the relative ADC contrasts, the tumor has relatively low water mobility in each evaluated brain region on each of the ADC map derived from the different $b$-values which is more apparent on the ADC map derived from high b-values (i.e. ADC4k). The $i \S A D C R_{i}{ }^{*}$ map confirms the increased degree of multiexponential contrast in white matter

CONCLUSIONS

Normal brain and human glioma demonstrate substantial multiexponential diffusion decay properties observable at high b- values. Highly perfused cellular tumor has multiexponential characteristics similar to, or greater than white matter. Even in hypoperfused tumor strong multiexponential behavior persists. This suggests even necrosis behaves as a complex multicompartment or multi-viscosity diffusion system.

\section{SL01:5:11}

\section{INCLUSION OR EXCLUSION OF INTRA-TUMOURAL VESSELS FOR RELATIVE CEREBRAL BLOOD VOLUME (RCBV) CALCULATIONS IN LOW -GRADE GLIOMAS: DOES IT MAKE A DIFFERENCE?}

\author{
G. Brasil Caseiras ${ }^{1,2}$, J. Thornton ${ }^{2}$, \\ T. Yousry ${ }^{1,2}$, J. Rees ${ }^{3}$, A. Waldman ${ }^{4}$, H. Jäger $^{1,2}$ \\ ${ }^{1}$ Institute of Neurology, UCL, London, United Kingdom; \\ ${ }^{2}$ Lysholm Department of Neuroradiology, UCL, London, United \\ Kingdom; ${ }^{3}$ National Hospital for Neurology and Neurosurgery, \\ London, United Kingdom; ${ }^{4}$ Department of Imaging, Charing Cross \\ Hospital, London, United Kingdom
}

\section{PURPOSE}

To assess the influence of intratumoural vessels on the calculation of rCBV (relative cerebral blood volume) in low-grade gliomas (LGG) and their impact on the differentiation between astrocytomas (ACs), oligodendrogliomas (ODs) and oligoastrocytomas (OAs). METHODS

34 patients with LGG (21 ACs; 8 ODs and 5 OAs) had dynamic susceptibility contrast enhanced $\mathrm{T} 2 *$-weighted perfusion imaging. Color maps of rCBV were generated. Two different methods for calculating the maximum intra-tumoural $\mathrm{rCBV}$ ( $\mathrm{rCBVmax}$ ) were used: method A included and method B excluded blood vessels from the intra-tumoural ROIs. Blood vessels were identified on simultaneous inspection of unprocessed source data of the perfusion series. For each method, the highest of several intratumoural rCBV measurements was normalised with the CBV of the contra-lateral normal appearing white matter. Wilcoxon test was used to assess differences in mean rCBVmax between the two methods and ordinal regression was used to assess the relationship of rCBVmax and the tumour histology.

\section{RESULTS}

Method A yielded overall significantly higher rCBVmax than Method B (4.01 versus 1.63, p value $<0.001)$. The values for $\mathrm{AC}$, $\mathrm{OA}$ and $\mathrm{OD}$ were $3.30,5.33$ and 5.04 with method $\mathrm{A}$ and 1.63 , 1.53 and 2.21 with method B. A significant association between rCBVmax and all three histological subtypes could only be demonstrated when using method B, but not with method A. With method $\mathrm{B}$ the odd ratio of being in a different category was 4.22 for each additional rCBV value. 


\section{CONCLUSION}

Inclusion of intratumoural vessels significantly alters the $\mathrm{rCBV}$ in all types of LGG. Excluding intratumoural vessels for $\mathrm{rCBV}$ measurement provides a better discrimination between histological subtypes of LGG. These results also highlight he importance of standardizing the method of $\mathrm{rCBV}$ measurement in multi-centre studies.

\section{SL01:5:12}

\section{MULTIFOCAL TUMOROUS BRAIN LESIONS:} METASTASIS OR GLIOBLASTOMA MULTIFORME (GBM)?

\section{S. Ulmer $^{1}$, O. Jansen ${ }^{1}$, F. Barker ${ }^{2}$, J. Henson ${ }^{3}$}

1 Section of Neuroradiology, Department of Neurosurgery, University Hospital of Schleswig-Holstein, Kiel, Germany; ${ }^{2}$ Neurosurgical Service, Massachusetts General Hospital, Boston, USA; ${ }^{3}$ Pappas Center for Neuro-Oncology, Massachusetts General Hospital, Boston, USA

\section{PURPOSE}

Malignant gliomas have been reported to be multifocal in 5 to $15 \%$ of cases. They are less likely to be multifocal at the time of diagnosis than are metastatic tumors, a fact that is often used to predict the etiology of newly detected mass lesions. However, the incidence of multifocality in malignant gliomas has not been well characterized using MRI.

\section{METHODS AND MATERIALS}

We retrospectively reviewed preoperative MRI scans of 141 patients with enhancing, newly detected malignant glioma, and determined the incidence of multifocal lesions, which was compared to the incidence of multifocal lesions in a comparison group of 88 patients with brain metastases. The nature of the multifocal lesions was also characterized. Imaging sequences had to include a T1-weighted sequence $+\mathrm{Gd}$ and a T2-weighted sequence (i.e. FLAIR) before resection of the tumor.

\section{RESULTS}

Overall 95 glioblastomas could be included in the study. Of these $65 \%$ were single lesions. Multifocal lesions were present in $35 \%$ of patients with GBM. 55/88 (58\%) patients with newly diagnosed brain metastasis had multifocal lesions (difference significant at $\mathrm{p}$ $<0.001$ ). Discrete enhancing foci within a region of contiguous T2-weighted hyperintensity were seen in 19\% of GBM. Entirely separate foci were present in $12 \%$ of GBM. In metastasis the pattern of entirely separate foci was much more common $(19 \%)$ than being multifocal with a connecting T2-abnormality (2\%).

CONCLUSIONS

Multifocal lesions are more common in patients with newly detected malignant glioma than is currently understood. The pattern of a contiguous T2-abnormality between lesions can distinguish GBM from metastasis.

\section{SL02}

12.50-13.30 - Interventional: brain, aneurysms and malformations

\section{SL02:6:1}

\section{RUPTURED DISSECTING ANEURYSM ARISING FROM THE POSTERIOR CIRCULATION ARTERIES: ENDOVASCULAR PERSPECTIVE}

I. Oran, C. Cinar

Ege University Medical School, Izmir, Turkey

\section{PURPOSE}

Most intracranial dissecting aneurysms involve the posterior circulation. Indications, techniques, and results of endovascular treatment in patients with ruptured posterior circulation aneursm are discussed.

\section{METHODS}

During last 7 years, 23 patints with SAH related to dissecting aneurysm arising from the posterior circulation arteries were rewieved retrospectively. The age range was 7 to 65 years (mean, 48 years). Ninteen patients were admitted in Hunt-Hess grades I and II, three were in grade III, and two were in grade IV.

\section{RESULTS}

Angiography showed dissecting aneurysm in posterior circulation in all patients: 8 posterior cerebral artery, 5 superior cerebellar artery, 5 basilar artery trunk, 4 posterior inferior cerebellar artery, and 1 basilar artery-superior cerebellar artery junction aneurysm. The angiographic features of aneursm were divided into following groups: pearl and string sign, 9 aneursms; fusiform dilatation, 9 aneurysms; double lumen, 5 aneursms. Besides 23 dissecting aneursms in 23 patients, additional cerebellar AVM in one patient, and multiple distal AICA aneursms in one other patient were also discovered. Endovascular treatment was performed in the acute stage in 21 patients. It was delayed (at 5 th week) for 2 patients. Aneurysm occlusion with preservation of parent artery was possible in 4 acute patients. In the remaining acute patients, during aneurysm embolization parent artery occlusion was inevitable. The 2 chronic patients (basilar artery aneursm) were treated with stent assissted coil embolization. There were two technical complications occurring during endovascular treatment (thromboemblisms) which did not resulted in clinical consequences. There were two permenant (hemiparesia-GOS 3 and homonim hemianopsia) and two transient (cerebellar syndrom and ninth cranial nevre paralysis) neurologic complications. One patient rebled and died 20 days after initial treatment. In the follow-up, two recanalization occurred; one was re-embolized successfully with preservation of parent artery, the other aneurysm (basilar artery) was embolized with parent artery occlusion which resulted in hemiparesia (GOS 1).

\section{CONCLUSION}

Embolization with or without parent artery occlusion is feasible with acceptable morbidity and mortality rate in the treatment of dissecting aneurysm confined in the posterior circulation. 


\section{SL02:6:2}

\section{VERTEBRAL ARTERY PSEUDOANEURYSM: SPONTANEOUS HEALING SUSPENDING ANTICOAGULATION THERAPY}

\section{G. Callari ${ }^{1}$, M. Furlan ${ }^{3}$, R. Padolecchia ${ }^{2}$,}

C. Serrati ${ }^{3}$

1 Neuroradiology of Imperia - Ponente Ligure Neurosciences Department, Imperia, Italy; ${ }^{2}$ Neuroradiology of Pietra Ligure (SV) - Ponente Ligure Neurosciences Department, Pietra Ligure (SV), Italy; ${ }^{3}$ Neurology of Imperia - Ponente Ligure Neurosciences Department, Imperia, Italy

We present the case of a 37 years old man with a symptomatic right vertebral artery dissection treated with anticoagulation therapy. On a 2 months follow up angio MRI, an intracranial pseudoaneurysm was detected in the site of dissection so that anticoagulation therapy was suspended. An angiogram (DSA) performed 2 weeks lather showed the correct filling of the right vertebral artery and the healing of the pseudoaneurysm.

We suggest that even if an intracranial pseudoaneurysm requires an aggressive treatment because of its risk of bleeding, the suspension of anticoagulation therapy and a diagnostic angiogram after 2 weeks, should be considered to detect a spontaneous healing.

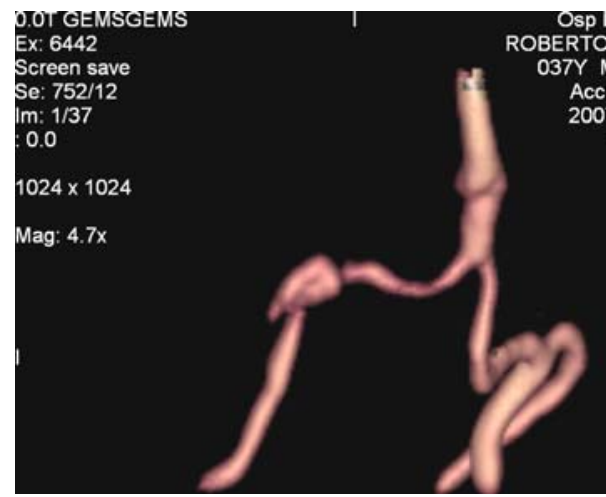

\section{SL02:6:3}

\section{A NEW DETACHABLE-TIP MICROCATHETER IN ENDOVASCULAR TREATMENT OF VASCULAR MALFORMATIONS: PRELIMINARY CLINICAL EXPERIENCE}

$\underline{\text { K. Yavuz }}^{1}$, S. Geyik ${ }^{1}$, U. Belet ${ }^{2}$, S. Cekirge ${ }^{1}$, I. Saatci ${ }^{1}$

${ }^{1}$ Hacettepe University, Ankara, Turkey; ${ }^{2}$ On Dokuz Mayis University, Samsun, Turkey

\section{PURPOSE}

To report the preliminary clinical experience with a new microcatheter (Sonic; Balt, Montmorency, France) which has a detachable tip zone in prolonged Onyx injections in intracranial/ spinal vascular malformations.

\section{METHODS}

Twenty-eight patients (22 male and 6 female) with an age range of 7 months-57 years were treated for intracranial/spinal vascular malformations using prolonged intranidal Onyx injection technique. Of these 28 patients, 25 were cerebral AVMs, 2 were spinal AVM/AVF and one was dural AVF. Sonic microcatheter was used for single injections for all patients but three and was intended to be withdrawn in all patients at the end of the procedures. In 3 patients 2 microcatheters were used for seperate injections.

RESULTS

Microcatheter sizes with detachable tip zone lengths were $1.2 \mathrm{~F}$ with $25 \mathrm{~mm}(15), 1.2 \mathrm{~F}$ with $15 \mathrm{~mm}(4), 1.5 \mathrm{~F}$ with $25 \mathrm{~mm}$ (10) and $1.5 \mathrm{~F}$ with $15 \mathrm{~mm}$ (1). In one patient, the microcatheter was ruptured within the detachable zone inside the feeder of the AVM during Onyx injection. Injection was continued for several minutes afterwards since the penetration of Onyx was still intranidal. Including this ruptured one, all the microcatheters were detached from the detachment marker and withdrawn at the end of the procedure without any difficulties. No other procedural complications occured.

CONCLUSION

Gluing of the microcatheter tip in prolonged intranidal Onyx injections is a concern in endovascular treatment of vascular malformations. This initial experience with the new detachable-tip microcatheter revealed promising results; however, future experience with a larger number of patients may better define the safety and efficacy of this new device.

\section{SL02:6:4}

\section{ENDOVASCULAR TREATMENT OF CRANIAL DAVFS WITH SPECIAL EMPHASIS ON ACTIVE SQUEEZE FINISHING}

J. Fiehler, T. Illies, U. Grzyska, H. Zeumer

Department of Neuroradiology, University Medical Centre, Hamburg, Germany

\section{BACKGROUND}

Endovascular techniques for exclusion of cranial dural arteriovenous fistulae (dAVFs) include transarterial and transvenous embolization by means of coil placement and injection of particles or liquid embolic material, as well as stent placement of a in the involved sinus.

\section{PATIENTS AND METHODS}

We reviewed our local database of the patients with cranial dural fistulae from 01/2003. Primary intended approach was the occlusion of the fistula point from the venous side. In case of trapped dAVF inaccessible via the venous route, having outflow only via tortous epicerebral veins we suggest arterial embolization of all feeders, leaving the largest and most straight one going to the fistula to be the last remaining. This is preferrably in the middle meningeal artery. In a final step, the microcathether (usually Tracker 18 or flow directed) is navigated distally into a wedge position. Thus the glue is safely injected onto the fistolus venous pouch and not flushed away by collateral flow. 
We use the term active squeeze finishing" for this procedure. This technique can be used as primary and secondary approach.

\section{RESULTS}

In total, 43 dAVF patients ( 25 male, 18 female) were identified, mean age was 57 years. 32 dAVFs were treated endovascularly, 6 were treated by open neurosurgery, and 5 were left untreated. Endovascular treatment was performed solely from the venous side by coils in $28 \%$, in a transorbital approach via the superior ophthalmic vein in 3\%, from the venous side in combination with arterial embolization in $31 \%$, and solely from the arterial side in $38 \%$ of the cases. The active squeeze finishing technique was used in 8 patients, predominantly in cases with trapped fistuale that could not be reached on a venous route. In total complete exlusion of the fistula could be achieved in $67 \%$, subtotal occlusion was recorded in $33 \%$ of the patients. After active squeeze finishing complete occlusion was observed in $7 / 8$ patients $(88 \%)$.

DISCUSSION

In principle, the path to the venous origin of the dAVF as prerequisite for an endovascular approach is determined by its individual anatomy. The active squeeze finishing technique is a promising alternative if a venous access is missing and a complex fistula has to be occluded by any means. In our small series a good occlusion rate was observed.

\section{SL02:6:5}

\section{INTRAOSSEOUS HEMANGIOMAS OF THE FACE: ENDOVASCULAR TREATMENT IN THE PEDIATRIC AGE GROUP}

C. Gandolfo $^{1}$, L. Castellan ${ }^{2}$, G. Morana ${ }^{1}$, A. Rossi ${ }^{1}$

${ }^{1}$ Department of Pediatric Neuroradiology, G. Gaslini Children's Research Hospital, Genoa, Italy; ${ }^{2}$ Department of Neuroradiology, S. Martino Hospital, Genoa, Italy

\section{PURPOSE}

Intraosseous hemangiomas are rare, benign vasoformative neoplasms (or developmental conditions of endothelial origin). They areparticularly uncommon in the pediatric age group, where they mainly occur in the second decade of life. We aimed to present a series of 3 children with intraosseous hemangioma affecting the bones of the face ( 2 of the maxilla and 1 of the mandibular bone) who were treated at our institutions in the past three years.

\section{MATERIAL AND METHODS}

Presenting symptoms included presence of painless mass (2 cases), spontaneous hemorrhage (1 case), bleeding secondary to mastication ( 1 case), and compromised tooth eruption ( 1 case). All three patients underwent neuroradiological assessment with CT and MRI, and MRA and were eventually diagnosed with capillary hemangiomas. In two cases, biopsy was performed at an outside institution, which precipitated moderate to important bleeding but also allowed histological verification.

\section{RESULTS}

In all cases, in order to control and prevent further hemorrhage and to assess the presence of high or low flow vascular patterns, we performed digital angiography followed by embolization resulting in immediate arrest of the hemorrhage. A satisfactory outcome was confirmed over a variable follow-up period (ranging from 6 months to 2 years), and all patients no longer complain with bleeding problem even during chewing.

\section{CONCLUSION}

The choice and timing of the definitive multidisciplinary treatment, aiming to correct the mass effect, remodel the bone deformity, and improve the cosmetic appearance, is still under debate. In our experience, presurgical endovascular embolization was a useful and safe procedure to reduce intraoperative bleeding risk. We therefore advocate its usefulness also before bioptic attempts.

\section{SL03}

15.50-16.55 - Interventional: brain, aneurysms

\section{SL03:7:1}

\section{RESULTS OF THE ATENA STUDY REGARDING THE ENDOVASCULAR TREATMENT OF UNRUPTURED INTRACRANIAL ANEURYSMS}

$\underline{\text { L. Pierot }}^{1}$, L. Spelle ${ }^{2}$, F. Vitry ${ }^{1}$

${ }^{1}$ Hôpital Maison-Blanche, CHU Reims, Reims, France;

${ }^{2}$ Fondation Rotschild Paris, France

\section{PURPOSE}

The diagnostic and therapeutic management of unruptured intracranial aneurysms is still a matter of debates. The French Society of Neuroradiology has conducted a registry (ATENA) to precisely evaluate the clinical and anatomical results of the Endovascular Treatment of Unruptured Intracranial Aneurysms. METHODS

ATENA is a prospective, multicenter, consecutive registry. From June 2005 to October 2006, 651 patients harbouring 1101 aneurysms were included in 27 French centers. 741 aneurysms were treated. Most patients included in this series were females (78\%) and 41 to 60 years old $(63 \%)$.

\section{RESULTS}

Treatment failed in $4.4 \%$ of cases. Technical complications were encountered in $16.6 \%$ of patients, including peroperative rupture (2.6\%), thromboembolism $(7.7 \%)$, device related complications $(2.9 \%)$ and others $(4.4 \%)$. At one month of the treatment, morbidity was $2.2 \%$ and morbidity $1.1 \%$. Cases of deaths were peroperative rupture in 3 patients, thromboembolism followed by intracerebral bleeding in 2 patient and intracerebral not related to the aneurysm treated in 2 cases. At one month morbidity was $2.2 \%$ and mortality $1.1 \%$. Factors affecting morbidity and mortality are analyzed.

\section{CONCLUSION}

ATENA is the largest prospective, multicentric study dealing with the endovascular treatment of unruptured intracranial aneurysms. 
Morbimortality of this treatment is low. According to this result, endovascular treatment will have an increasing place in the treatment of unruptured intracranial aneurysms.

\section{SL03:7:2}

\section{SAFETY AND EFFICACY OF MATRIX COILS \\ FOR THE SELECTIVE ENDOVASCULAR TREATMENT OF INTRACRANIAL ANEURYSMS}

\author{
L. Pierot $^{1}$, X. Leclerc ${ }^{2}$, A. Bonafe ${ }^{3}$, \\ S. Bracard ${ }^{4}$ \\ ${ }^{1}$ CHU Reims, Reims, France; ${ }^{2}$ CHU Lille, Lille, France; \\ ${ }^{3}$ CHU Montpellier, Montpellier, France; ${ }^{4}$ CHU Nancy, Nancy, \\ France
}

\section{OBJECTIVES}

To evaluate the clinical and anatomical results of the selective endovascular treatment of intracranial aneurysms using Matrix coils.

\section{METHODS}

From January 2004 to October 2004 , a prospective, multicenter registry was conducted in 16 french centers. 236 patients (females: 149, males: 87 ; age : 21 to 78 years; mean: 48.7 years) harboring 244 aneurysms were included. Aneurysms were located in anterior circulation in $93 \%$ and posterior circulation in $7 \%$. Aneurysmal occlusion was evaluated by an independent core laboratory. Evaluation was performed postoperatively in all cases and 9 to 18 months after the treatment in 144 cases.

RESULTS

Technical and clinical preoperative complications were encountered in $18 \%$ of cases. Postoperative morbidity and mortality related to the treatment were respectively $2.5 \%$ and $0.8 \%$. At one year follow-up, 89 out of 103 patients with ruptured aneurysms (86.5\%) had a modified Rankin Score (mRS) from 0 to 2; in unruptured group, mRS was 0 to 2 in $99 \%$ of patients.

Postoperatively, classification by the core lab was complete occlusion in $44 \%$, neck remnant in $25 \%$ of cases and aneurysm remnant in $31 \%$ of cases. At one year, occlusion was complete in $47 \%$ of cases, neck remnant in $26 \%$ of cases and aneurysm in $28 \%$ of cases according to the core lab analysis.

At one year the recanalization rate was $26 \%$. An improvement of the occlusion rate was observed in $30 \%$ at one year. Factors affecting anatomical evolution of aneurysms treated with Matrix coils are reported.

\section{CONCLUSION}

The rate of complications, morbidity and mortality in patients treated with Matrix coils is not higher than in patients treated with bare coils. Recanalization is observed in $26 \%$ of cases. Subsequent occlusion after Matrix treatment is reported in $30 \%$ of cases.

\section{SL03:7:3}

PRELIMINARY RESULTS OF CLARITY STUDY: PLACE AND RESULTS OF THE ENDOVASCULAR TREATMENT OF RUPTURED ANEURYSMS USING GDC COILS

L. Pierot $^{1}$, C. Cognard ${ }^{2}$

${ }^{1}$ Hopital Maison-Blanche - Chu Reims, Reims, France;

${ }^{2} \mathrm{Chu}$ Toulouse, Toulouse, France

\section{OBJECTIVES}

To evaluate place and the results of the endovascular treatment In the management of ruptured intracranial aneurysms.

\section{METHODS}

A prospective, multicentric registry was started in France in October 2006 in 21 neurointerventional centers to evaluate precisely the place and the results of the endovascular treatment in the management of ruptured intracranial aneurysms and to compare safety and efficacy of GDC and Matrix coils. In a first part aneurysms are treated with GDC coils. In the second part, aneurysms will be treated with Matrix coils. The objective is to include approximately 400 patients in each group (GDC and Matrix).

\section{RESULTS}

At the end of March 2007, approximately 300 patients have been included in the GDC part of the study. Inclusion in GDC group will come to end in May 2007. Methology of the study and a first analysis of the results in GDC group will be presented.

\section{CONCLUSION}

Since ISAT results have been published, no precise evaluation of the place and results of the endovascular treatment of ruptured intracranial aneurysms has been performed. CLARITY is a prospective, multicentric consecutive conducted in France to evaluate the place and results of the endovascular treatment in the management of ruptured intracranial aneurysms an to compare safety and efficacy of GDC and Matrix coils. Preliminary results regarding the place and results of the endovascular treatment using GDC coils will be presented.

\section{SL03:7:4}

\section{MIDTERM CLINICAL AND ANGIOGRAPHIC RESULTS OF ENDOVASCULAR ANEURYSM TREATMENT WITH CERECYTE COILS:SINGLE CENTER EXPERIENC}

S. Geyik ${ }^{1}, \underline{\text { K. Yavuz }}^{1}$, O. Koc ${ }^{2}$, I. Saatci ${ }^{1}$, S. Cekirge

${ }^{1}$ Hacettepe University, Ankara, Turkey; ${ }^{2}$ Konya Selcuk University, Konya, Turkey

\section{PURPOSE}

To evaluate midterm clinical and angiographic results of the new bioactive "Cerecyte" coil. 


\section{METHODS}

This series include eighty cerebral aneurysms in 74 patients treated with Cerecyte coils in a single center excluding the aneurysms randomized for the ongoing multicenter trial. Six aneurysms were large, 73 were small and one was partially thrombosed giant. Fifty-eight aneurysms were wide-necked. Sixtyseven aneurysms were located in anterior and 13 in posterior circulation. Forty four aneurysms were ruptured. All patients but 3 were treated using balloon remodeling technique. Sixty-six aneurysms (in 61 patients) were available for follow-up. Six months control angiography was performed for 38 aneurysms, 1 year control for 24 aneurysms (in 6 patients with MRA), 1.5 year for one aneurysm with MRA and 2 years for 3 aneurysms with angio.

\section{RESULTS}

Complete occlusion (Raymond class 1) was achieved in 57 aneurysms, class 2 in 22, and class 3 in one aneurysm in the postoperative angiography. Thromboembolic event occurred in 5 patients causing morbidity in one. According to the latest followup, among the 53 aneurysms with class 1 occlusion, 2 were changed to class 2 , and one to class 3 . Of the 17 aneurysms with class 2 occlusion 13 showed progressive occlusion, 2 stayed stable and 2 were changed to class 3 which needed retreatment. Overall, regrowth rate was $7.5 \%(5 / 66$ aneurysms) in the group with follow-up. Four patients (5.4\%) died; being procedure related in one $(1.3 \%)$. Permanent morbidity occured in one patient.

CONCLUSION

Our initial procedural data demonstrates that Cerecyte coils are safe and mid-term follow-up results showed a favorably low recanalization rates.

\section{SL03:7:5}

\section{INTRACRANIAL ANEURYSMS TREATMENT USING MATRIX COILS: THREE YEARS OF EXPERIENCE AND FOLLOW-UPS RESULTS.}

N. Nuzzi, M. Isalberti, C. Cinnante, C. Sina, A. Costa, S. Avignone, P. Machì, V. Branca

Neuroradiologia Diagnostica ed Interventistica Fondazione Ospedale Maggiore Policlinico Mangiagalli Regina Elena - IRCCS Milano, Italy

\section{INTRODUCTION}

Matrix coils (Boston Scientific) were the first coils made in order to face the problem of recurrence in coiled aneurysms trying to associate the pharmacological treatment with the mechanical exclusion of the sac. Aim of this study is to weight follow-up data nowadays available regarding aneurysms treated in our centre with Matrix, because at high risk of recurrence or because already recurrent.

\section{MATHERIAL AND METHODS}

Matrix coils were used to treat small aneurysms with wide neck, large (over $10 \mathrm{~mm}$ of diameter), giant (over $25 \mathrm{~mm}$ ), and to treat recurrent aneurysms. Whenever it was possible, only Matrix coil were used; in some cases bare metal coils (GDC - Boston
Scientific) were associated in order to obtain a better packing of the sac. Only aneurysms filled with Matrix coils, for at least 50\% of total length of the coil ball, were included in this study.

From January 2004 to February 2007141 aneurysms were treated (90 small, 45 large, and 6 giant) in 122 patients (88 female, 34 male) in a 23-78 age range; 16 aneurysms were recurrence of coiling, 1 case of clipping.

16 patients with 18 aneurysms were lost at the follow-up; 1 patient died of SAH; 13 patients with 15 aneurysms are waiting followup. Angiographic follow-up data are available for 107 aneurysms in 92 patients from 3 months up to 3 years (63 small, 38 large, 6 giant).

\section{RESULTS}

In few cases ( 21 of 141 , mostly small aneurysms) was possible to use only Matrix coils; sometimes GDC coils were associated during coiling, due to compartmentalisation and friction between Matrix coils, in order to reach a more dense occlusion. Although complete occlusion was reached, in large and giant aneurysms empty spaces were often recognisable.

30 aneurysms did recur: 8 were small (12,6\%), 17 large (44,7\%), and 5 giant $(83 \%) .21$ of them $(19,6 \%)$ were retreated.

Recurrence was almost due to coil ball compaction and not to aneurysm growth.

\section{CONCLUSIONS}

Matrix coils did not prevent new inflow in large and giant aneurysms with high risk of recurrence; the possible effect of the Matrix coils bioactive coating seems reduced by coil ball compaction that happen after it has melted away. Randomised study is necessary to assess the real benefit of Matrix coils versus bare metal coils.

\section{SL03:7:6}

\section{EFFECTIVENESS AND SAFETY OF TIROFIBAN DURING COILING OF INCIDENTAL CEREBRAL ANEURYSMS}

$\underline{\text { S. Mueller-Schunk }}{ }^{1}$, J. Lewandowski ${ }^{1}$, M. Wiesmann ${ }^{1}$, R. Schmid-Elsaesser ${ }^{2}$, T. Pfefferkorn ${ }^{3}$, P. Moehnle ${ }^{4}$, H. Brueckmann ${ }^{1}$, T. Mayer ${ }^{1}$

${ }^{1}$ Dept. of Neuroradiology, Klinikum Grosshadern, LMU, Munich, Germany; ${ }^{2}$ Dept. of Neurosurgery, Klinikum Grosshadern, LMU, Munich, Germany; ${ }^{3}$ Dept. of Neurology, Klinikum Grosshadern, LMU, Munich, Germany;

${ }^{4}$ Dept. of Neuroanesthesiology, Klinikum Grosshadern, LMU, Munich, Germany

\section{PURPOSE}

To analyze periprocedural complications in endovascular coiling of incidental cerebral aneurysms treated with tirofiban and to determine its safety and effectiveness to prohibit or treat thrombembolic complications.

\section{METHODS}

63 coil embolization procedures of unruptired aneurysms in 61 patients were analyzed retrospectively. Angiograms, periinterven- 
tional CT scans or MRIs, and clinical records were evaluated for intracranial bleedings, thrombembolic complications, and all procedure related clinical complications.

\section{RESULTS}

In 5/61 (8\%) patients an intraprocedural thrombus at the aneurysm neck had occurred despite platelet inhibition with aspirin. In all 5 cases the thrombus could be resolved with tirofiban under DSA control. In $35(49 \%)$ patients tirofiban was given because of stenting. $3 / 35(8.5 \%)$ patients developed in stent thrombosis despite aspirin and tirofiban treatment in standard dose. 0/61 patients suffered from an intracerebral bleeding. In one case aneurysm rupture under tirofiban lead to severe $\mathrm{SAH}$, but the patient recovered to mRS 1 after 2 years. One patient suffered from aneurysm rupture before tirofiban application but had no rebleeding under tirofiban. 3/61 (5\%) patients had extracranial bleedings under tirofiban. Two developed groin hematoma, one suffered from intrapulmonal bleeding. $5(8 \%)$ patients showed thrombocytopenia. In one patient an allergic reaction to tirofiban was in question.

\section{CONCLUSIONS}

The use of tirofiban for coiling of incidental aneurysms seems safe. It can help to treat thrombembolic complications, but cannot always prevent stent-thrombosis. This may be due to individual responses to tirofiban. The effectiveness of tirofiban should therefore be monitored. Hemorrhagic complications do occur rarely, but were of low impact in longterm clinical outcome in this study.

\section{SL03:7:7}

\section{EMBOLIZATION OF CEREBRAL ANEURYSM ASSOCIATED WITH VASOSPASM: ARTERIAL INFUSION OF NIMODIPINE THROUGHOUT THE PROCEDURE}

\author{
I. Oran, C. Cinar \\ Ege University Medical School, Izmir, Turkey
}

\section{PURPOSE}

Patients who have already developed vasospasm, are prone to (thromboembolic) complication during aneurysm embolization. We evaluated the effectiveness of combined embolization of aneurysm and intra-arterial nimodipine infusion which continues during entire procedure.

METHODS

During last three years, 10 patients with ruptured aneurysms associated with vasospasm were treated in a single session. After initial intra-arterial nimodipine infusion (1-2 $\mathrm{mg}$ within $10-15$ minutes), they underwent aneurysm occlusion with coils under continuous intra-arterial infusion of nimodipine at a dose of $1 \mathrm{mg} / \mathrm{h}$. RESULTS

Angiography showed moderate to severe proximal and distal vasospasm in 9 patients, and only distal (to aneurysm) vasospasm in one patient. Complete aneurysm occlusion was achieved in 6 patients, incomplete occlusion in 3 patients, and small neck remnant in one patient. Final angiogram also demonstrated complete clearance of spasm in 6 patients, complete proximal and significant distal clearance in one, and significant distal clearance in one patient. There was no medical complication related to the extended nimodipine infusion nor technical complication.

CONCLUSION

Extended intra-arterial nimodipine infusion up to the end of embolization procedure is effective and safe in patients with ruptured aneurysm and associated vasospasm. This technique seems to increase security of the procedure as well as force further vasorelaxation when endovascular route is used to treat both aneurysm and vasospasm in a single step. Further prospective studies are needed to confirm these results.

\section{SL03:7:8}

\section{LONG TERM FOLLOW UP OF CEREBRAL ANEURYSMS AFTER ENDOVASCULAR THERAPY - PREDICTION AND OUTCOME OF RETREATMENT}
T. Ries ${ }^{1}$, S. Siemonsen ${ }^{1}$, G. Thomalla ${ }^{2}$, U. Grzyska ${ }^{1}$, H. Zeumer ${ }^{1}$, J. Fiehler ${ }^{1}$
1 Department of Neuroradiology, University Medical Centre, Hamburg, Germany; ${ }^{2}$ Department of Neurology, University Medical Centre, Hamburg, Germany

\section{PURPOSE}

To analyze angiographic and clinical results prior and after additional endovascular therapy in patients with previously coiled but reopened cerebral aneurysms and to identify possible risk factors for aneurysm retreatment.

PATIENTS AND TECHNIQUES

Follow up with selective digital subtraction angiography was performed in 323/596 (54.2\%) patients harboring 342 aneurysms with a mean follow up time of 28.6 months. The patients were divided into three groups, those who remained stable after initial treatment (A), those who showed minor morphological changings (B) and those who underwent repeat treatment (C). The decision whether an aneurysm was to be retreated was always made on the basis of multiple projection images (DSA) by the most senior neuroradiologist available in the angio-lab. The major criterion for the decision for retreatment was the exposure of aneurysm sac uncovered by coils resulting in a recurrence of more than $2 \mathrm{~mm}$ in order to provide an appropriate cavity for an additional coil placement. Univariate and multivariate regression analysis were performed to determine possible risk factors for aneurysm retreatment. A significant number of patients were lost to clinical follow up (22.7\%).

\section{RESULTS}

Single or multiple retreatment was performed in 33/323 patients (10.2\%). Mean angiographic follow up period for these patients was 28.6 (range 6-132) months. Retreatment of small aneurysms $(<=10 \mathrm{~mm})$ with small necks $(<=4 \mathrm{~mm})$ was performed in $6 / 214$ aneurysms. When summarizing all other aneurysms as "risk group" $(\mathrm{n}=128)$, the odds ratio $(\mathrm{OR})$ for retreatment in the "risk group" was $3.11(95 \% \mathrm{CI}: 1.43-6.75 ; \mathrm{p}=0.004)$. In cases with 
residual aneurysm after first treatment, OR for retreatment was 3.96 (95\%CI: 1.48-10.65; p=0.006) whereas a neck remnant, clinical presentation and aneurysm localization were not predictive. We observed no resulting morbidity and mortality from 33 retreatment procedures.

\section{DISCUSSION}

The best predictors of aneurysm retreatment were aneurysm anatomy and presence of a residual aneurysm after initial treatment. Our data may suggest that complete treatment does not need not to be enforced by all means since neck remnants were not predictive for retreatment. However, due to a significant number of patients that were lost to follow up (22.7\%) the quality of our data is limited. A large multicenter database is needed to gather enough patients with the rare event of rebleeding in order to identify those patients who ultimately need a retreatment.

\section{SL04}

16.55-18.00 - The aging brain and neurodegenerative disorders I

\section{SL04:8:1}

\section{THE CONTRIBUTION OF NEURODEGENERATIVE AND VASCULAR PATHOLOGY TO COGNITIVE IMPAIRMENT IN VASCULAR DEMENTIA}

A. Bastos-Leite ${ }^{1}$, W. Van Der Flier ${ }^{2}$, E. Van Straaten ${ }^{2}$, S. Staekenborg $^{2}$, P. Scheltens ${ }^{2}$, F. Barkhof ${ }^{2}$

1 Faculdade de Medicina da Universidade do Porto, Oporto, Portugal; ${ }^{2}$ VU University Medical Center, Amsterdam, The Netherlands

\section{BACKGROUND AND PURPOSE}

Besides cerebrovascular disease, medial temporal lobe atrophy (MTA), a neuroimaging finding suggestive of degenerative pathology, has been shown in vascular dementia. However, it is unknown to what extent neurodegenerative pathology contributes to cognitive impairment in vascular dementia. Therefore, our purpose was to investigate the relative contribution of cerebrovascular disease and MTA to cognitive impairment in patients fulfilling diagnostic criteria for vascular dementia.

METHODS

We examined 590 patients (374 men, mean age $=73$ years, standard deviation $=8$ ) with probable vascular dementia, according to the National Institute of Neurological Disorders and Stroke (NINDS) - Association Internationale pour la Recherche et l'Enseignement en Neurosciences (AIREN) criteria, at inclusion into a multicenter clinical trial. Cerebrovascular disease and the degree of MTA were evaluated by using magnetic resonance imaging. Cognitive testing included the mini mental state examination (MMSE), and the vascular dementia assessment scale (VaDAS-cog).

\section{RESULTS}

On the basis of the operational definitions for the neuroimaging part of the NINDS-AIREN criteria, 485 (82.2\%) patients had small vessel disease, and $153(25.9 \%)$ had large vessel vascular dementia. More than half $(59.8 \%)$ of the patients had considerable MTA. Multiple linear regression analyses revealed that after correction for sex, age, education, and duration of dementia, neuropsychological tests showed that patients with higher grades of MTA or large vessel vascular dementia had significantly worse general cognitive and executive functioning, while associations with small vessel disease were restricted to worse executive functioning.

\section{CONCLUSIONS}

Both MTA and large vessel disease contribute to global cognitive impairment in vascular dementia. Small vessel disease contributes to executive dysfunction.

\section{SL04:8:2}

\section{THE THALAMUS IN PROGRESSIVE SUPRANUCLEAR PALSY (PSP) AND CORTICO-BASAL DEGENERATION (CBD): DIFFUSION TENSOR IMAGING (DTI) STUDY.}

\author{
$\underline{\text { A. Erbetta }}{ }^{1}$, M. Mandelli ${ }^{1}$, M. Grisoli ${ }^{1}$, \\ A. Bizzi $^{1}$, T. De Simone ${ }^{1}$, P. Soliveri ${ }^{2}$, \\ F. Girotti ${ }^{2}$, M. Bruzzone ${ }^{1}$ \\ ${ }^{1}$ Dept. of Neuroradiology, IRCCS NEUROLOGICO C.BESTA, \\ Milan, Italy; ${ }^{2}$ Dept. of Neurology, IRCCS NEUROLOGICO \\ C.BESTA, Milan, Italy
}

\section{PURPOSE}

Progressive supranuclear palsy and cortico-basal degeneration may present with a similar clinical phenotype and share some neuroradiological findings. Neuropathological investigations show basal ganglia and thalami involvement in both diseases. The Fractional Anisotropy (FA) and Apparent Diffusion Coefficient (ADC) were measured in these structures to determine whether they are different involved in PSP and CBD by means of regions of interest.

\section{METHODS}

Nine patients with PSP (mean age 66 years), eleven with CBD (mean age 66 years) and twelve healthy controls (mean age 58 years) were studied. Regional ADCs and FAs were measured in the motor thalamus, in the medial and anterior thalamus, in supplementary motor area (SMA), in the primary motor area, in the anterior cingulate and in the pre-frontal area, in the globus pallidus, caudate nucleus and putamen. The ADC and FA values were analysed by means of one-way ANOVA with group as factor, followed by the Tukey post-hoc test.

RESULTS AND CONCLUSIONS

We found a significant ADC increase in medial thalamus (PSP: $0.84 \pm 0.03$, controls: $0.76 \pm 0.02, \mathrm{p}<0.01)$ and anterior thalamus (PSP: $0.82 \pm 0.07$, controls: $0.7 \pm 0.04, \mathrm{p}<0.001)$ and a significant FA decrease in anterior thalamus (PSP: $0.25 \pm 0.04$, controls: $0.30 \pm 0.02$, $\mathrm{p}<0.01$ ) in PSP patients. A significant ADC increase was found 
only in the motor thalamus (CBD: $0.75 \pm 0.05$, controls: $0.7 \pm 0.02$, p $<0.05)$ in $\mathrm{CBD}$ patients. In both diseases, the ADC values were significant increased in SMA (PSP: $0.76 \pm 0.05, \mathrm{p}<0.01$ CBD $0.78 \pm$ $0.06, \mathrm{p}<0.001$; controls: $0.68 \pm 0.04$ ), in the pre-frontal area (PSP: $0.76 \pm 0.07, \mathrm{p}<0.01$; CBD: $0.75 \pm 0.06, \quad \mathrm{p}<0.001$; controls: $0.67 \pm$ 0.03 ), in the primary motor area (PSP: $0.76 \pm 0.05, \mathrm{p}<0.01$; CBD: $0.8 \pm 0.05, \mathrm{p}<0.01$; controls: $0.7 \pm 0.03$ ), and in basal ganglia. These results show a major thalamus involvement in PSP patients.

Diffusion tensor imaging may represent a useful diagnostic tool that can provide additional support to discriminate PSP and CBD on the basis of thalamic ADC and FA values.

\section{SL04:8:3}

\section{VISUAL ASSESSMENT OF ATROPHY PATTERNS} IN ALZHEIMER DISEASE, FRONTOTEMPORAL DEMENTIA, AND PRIMARY PROGRESSIVE APHASIA

\author{
P. Vitali ${ }^{1,3}$, H. Shu ${ }^{1}$, D. Dean ${ }^{1}$, H. Rosen ${ }^{1}$, \\ M. Weiner ${ }^{2}$, B. Miller ${ }^{1}$, M. Gorno-Tempini ${ }^{1}$ \\ ${ }^{1}$ Memory and Aging Center, Neurology, UCSF, San Francisco \\ (CA), USA $;{ }^{2}$ Center for Imaging of Neurodegenerative Diseases, \\ VA Hospital, UCSF, San Francisco (CA), USA; ${ }^{3}$ Neurosurgery \\ and Neuroradiology, Istituto Neurologico C.Besta, Milano, Italy
}

Using Voxel-Based Morphometry (VBM), several studies (Boxer 2003, Gorno-Tempini 2003, Rabinovici 2007) have revealed unique patterns of cortical atrophy in groups of patients with Alzheimer Disease (AD), Frontotemporal Dementia (FTD), and three subtypes of Primary Progressive Aphasia (PPA): Semantic Dementia (SD), Progressive Non-Fluent Aphasia (PNFA), and Logopenic Progressive Aphasia (LPA). Few studies have evaluated whether visual, non-automated MRI readings are helpful in the differential diagnosis of these diseases. Recent analyses indicate that FTD might be particularly problematic as some cases do not show the predicted pattern of frontal atrophy (Kipps 2007).

In this study, visual inspection was performed on MRI scans from a cohort of AD, FTD, and PPA patients to determine the diagnostic utility of atrophy pattern recognition.

MRI scans were performed on 65 patients from the UCSF Memory and Aging Center cohort. Patients included in this cohort were distributed as follows: 13 AD, 13 FTD, 16 PNFA, 11 SD, 12 LPA. 30 cases were pathologically confirmed. Fifteen brain regions were selected for visual assessment based on previous VBM analyses.

Two blinded, experienced physicians assessed first the axial PDT2 images to evaluate the white matter abnormalities, then, to evaluate the cerebral atrophy, the T1 MPRAGE coronal images perpendicular to the main hippocampal axis, and two other reformatted planes. Each reader independently rated the degree of atrophy at each region using a simple 0-1-2 scale.

The 15 brain regions of interest were easily visualized on six anatomically-defined coronal reference planes and nearby images. In each case, atrophy was found in multiple brain regions.
SD cases were associated with severe temporal pole atrophy. The expected patterns of frontal atrophy in FTD and PNFA and the parietotemporal atrophy in AD and LPA was found in most cases. Quantitative analyses (linear and volumetric) will be used to objectively assess the visual rating results.

Fig 1: The six reference coronal planes, perpendicular to the main hippocampal axis, in a patient with semantic dementia and diiffuse atrophy, more severe in the right temporal lobe.

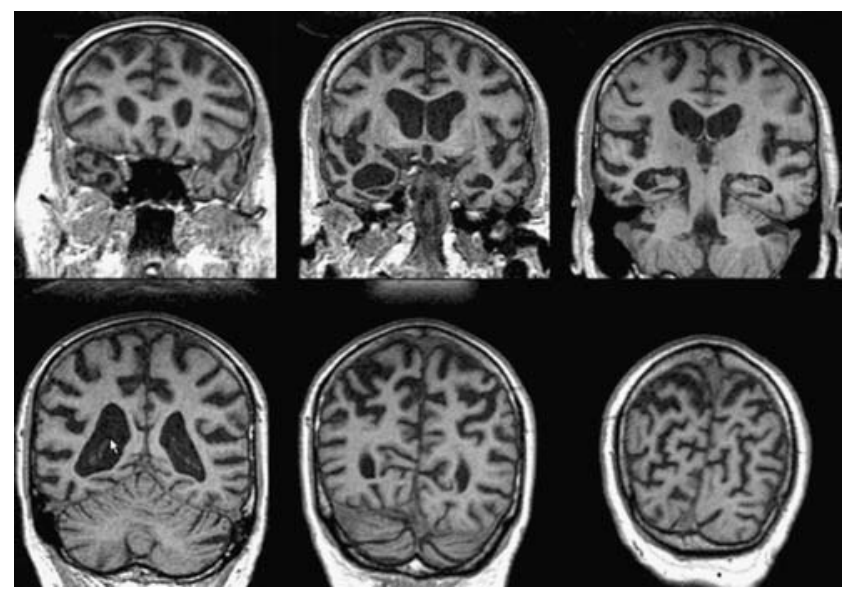

\section{SL04:8:4}

\section{EARLY DIAGNOSIS OF ALZHEIMER'S DISEASE: CONTRIBUTION OF DIFFUSION TENSOR IMAGING}

\author{
E. Scola ${ }^{1}$, A. Falini ${ }^{1}$, G. Magnani ${ }^{2}$, M. Filippi ${ }^{3}$, \\ M. Falautano ${ }^{4}$, E. Schiatti ${ }^{2}$, M. Mossini ${ }^{2}$, \\ G. Comi ${ }^{2}$, G. Scotti ${ }^{1}$ \\ 1 Neuroradiology Department and C.E.R.M.A.C. (Scientific \\ Institute and University Vita-Salute San Raffaele), Milan, Italy; ${ }^{2}$ \\ Neurology Department S. Raffaele Hospital, Milan, Italy; ${ }^{3}$ \\ Neuroimaging Research Unit (NRU) S. Raffaele Hospital, Milan, \\ Italy; ${ }^{4}$ Neuropsychology Unit S. Raffaele Hospital, Milan, Italy
}

\section{PURPOSE}

The aim of this study was to assess if MR DTI could help predicting the progression to $\mathrm{AD}$ in MCI patients.

Methods: 22 AD patients, $21 \mathrm{MCI}$ patients and 20 healthy subjects underwent MR DTI at baseline and a 2 years clinical follow up. Mean Diffusivity (MD) and Fractional Anisotrophy (FA) were calculated for the brain tissue (BT), the whole white matter (WM), the whole grey matter (GM ) separately and for GM and WM regions of interest (ROIs). Eleven MCI patients converted to $\mathrm{AD}$ during the follow up.

RESULTS

$\mathrm{MD}$ and FA calculated on BT, GM and WM were, respectively, higher and lower in MCI converters then in MCI non converters. ROIs analyses showed increased MD in MCI converters than in MCI non converters for most of the areas sampled, excepting middle cerebellar peduncles, genu of corpus callosum and anterior cingulate gyri, while lower FA was demostrated for few regions. However, only MD of WM was statistical different between the two groups (mean 
values 1.024 and $0.943 \mathrm{~m} 2 \mathrm{~s}-1 \times 10-9$ respectively, $\mathrm{p}=0.049)$. MCI converters showed significant differences in MD and FA of BT, GM and WM $(p=0.0001)$ and in MD of amygdalas $(p=0.048)$, hippocampi $(\mathrm{p}=0.001)$, temporal WM $(\mathrm{p}=0.039)$ and occipital WM $(\mathrm{p}=0.023)$ and $\mathrm{GM}(\mathrm{p}=0.021)$ compared with controls but not with $\mathrm{AD}$; on the contrary MD and FA of BT, GM and WM of MCI non converters were significant lower $(p<0.05)$ then those of AD while no statistical differences were seen with controls.

\section{CONCLUSION}

An early finding of medial temporal lobe and global WM damage, as shown by higher MD, could help predicting conversion to $\mathrm{AD}$ in MCI subjects. MD may correlate with functional changes in neurons and fibers while FA is associated with a reduction of microstructural integrity that occurs later in the natural history of AD pathology.

\section{SL04:8:5}

\section{DIFFUSION WEIGHTED IMAGING IN ALZHEIMERS DISEASE : WHOLE BRAIN AND REGIONAL ADC MEASUREMENTS.}

C. Micallef ${ }^{1}$, B. Ridha ${ }^{2}$, J. Shah ${ }^{1}$, N. Fox ${ }^{2}$, $\overline{\text { J. Thornton }}^{1,2}$, H. Jäger ${ }^{1,2}$

1 Lysholm Department of Neuroradiology, London, United Kingdom; ${ }^{2}$ Institute of Neuroradiology, London, United Kingdom

\section{AIM}

To investigate the roles of whole brain and regional apparent diffusion coefficient (ADC) measurements in differentiating patients with Alzheimers disease (AD) from age-matched controls. METHOD

11 patients with $\mathrm{AD}$ and 12 age-matched controls underwent diffusion weighted MR imaging with $b=1000$ s.mm-2. ADC maps were generated and after segmentation to excluded CSF and extraparenchymal tissue (FSL version 3.2), whole brain ADC histograms were produced. In addition the mean ADC from 17 regions of interest (ROIs) defined manually in various brain regions (frontal, parietal, temporal and occipital lobes, hippocampus, splenium, thalamus and pons) was recorded. Mean values for the $\mathrm{AD}$ and control groups were compared using the 2-sample t-test; Stepwise regression was used to determine the combination of measures which best differentiated between the 2 groups.

RESULTS

There were significant differences between patient and control groups for whole-brain mean ADC $(\mathrm{p}<0.001)$, and ADC histogram peak height $(\mathrm{p}=0.004)$, peak position $(\mathrm{p}=0.006)$ and positions of the 25th, 50th and 75th percentiles $(p=0.002,0.002$ and 0.001 respectively). In the ROI analysis, the $\mathrm{ADC}$ was significantly higher in $\mathrm{AD}$ patients with respect to controls in the parietal $(\mathrm{p}=0.001)$ and occipital $(p=0.004)$ lobes, hippocampus $(p=0.007)$ and thalamus $(p=0.05)$. A stepwise regression model revealed that, of the histogram measures, whole-brain mean ADC alone best differentiated between patients and controls $(p=0.013)$, while for the ROI measurements, the parietal lobe ADC sufficiently differentiated between patients and controls $(\mathrm{p}=0.011)$. Both mean whole brain
ADC and mean parietal lobe ADC were independently associated with case-control status after correction for the other factor using a multiple regression model.

\section{CONCLUSION}

Our results suggests that both mean whole-brain ADC and mean $\mathrm{ADC}$ in the parietal ROI provide complementary information of value in differentiating $\mathrm{AD}$ patients from controls.

\section{SL04:8:6}

\section{H-MRS AND STRUCTURAL MRI DIFFERENTIATE PRODROMAL ALZHEIMERS DISEASE (PRD-AD) FROM MCI, AD AND HEALTHY AGING CONTROLS}

B. Gomez-Anson ${ }^{1}$, G. Monte ${ }^{2}$, L. Rami ${ }^{3}$, J. Molinuevo ${ }^{3,4}$, R. Sanchez-Valle ${ }^{3}$, B. Bosch ${ }^{2}$

${ }^{1}$ Hospital Santa Creu I Sant Pau, Barcelona Spain;

${ }^{2}$ Fundacio Clinic, Barcelona Spain; ${ }^{3}$ Icmsn, Hospital Clinic, Barcelona Spain; ${ }^{4}$ Idibaps, Barcelona Spain

\section{INTRODUCTION}

Alzheimers disease (AD) results in metabolic and structural changes on MRI, different from normal aging. Recently, preclinical stages of $\mathrm{AD}$ have been defined on clinical-neuropsychological grounds, but there is little known about their characteristic MR features.

\section{OBJECTIVES}

To study the biochemical (1H-MRS) and structural MRI features in the brain of Prd-AD patients, compared to MCI, AD, and healthy aging controls.

Material and Methods: 100 Subjects (28 Prd-AD,14 aMCI, 31 $\mathrm{AD}$, and 27 controls), matched for age, sex and education. Detailed neuropsychology, and aMCI (Petersen's criteria), and AD diagnosis (DSM-IV and NINCDS-ADRA criteria; GDS 4-5). Prd-AD were defined as having: 1) ) memory decline on clinical judgement 2) significant episodic memory impairment 3) absence of other causes of memory disturbances 4) plus either: A) activities of daily living (ADL) impairment or B) additional cognitive domain significantly affected with preserved ADL. 1H-MRS (SVS, PRESS, $\mathrm{TR}=1500$; $\mathrm{TE}=30 ; 8 \mathrm{NEX}$ ) in 3 brain regions (Posterior CingulatedPC-, Left temporal pole -AP-, and posterior temporal-parietal -PT-), and 3D-IR SPGR axial MRI (Min TE, flip angle $=15, F O V=25 \times 20$, Thickness $=1.5,128$ locations, Matrix $=256 \times 192,1 \mathrm{NEX)}$ were obtained. Absolute metabolite concentration determinations (LCModel) and Voxel Based Morphometry (VBM) analysis were performed. Statistics included ANOVA, ANCOVA (age and sex as covariates), and post-hoc analysis (significance at $\mathrm{p}<0.05$ ).

RESULTS

Compared to aMCI and similarly to $\mathrm{AD}$, Prd-AD patients presented significantly lower $\mathrm{N}$-acetylaspartate (NAA), Creatine (Cre) and Glutamate values in the PC, and lower NAA in the TP. On VBM analysis, there was more prominent density loss in the left hippocampus in Prd-AD compared to MCI. AD patients had more prominent density loss than Prd-AD in the lateral, posterior temporal and parietal cortex. 


\section{CONCLUSIONS}

Prd-AD patients have characteristic metabolic and MRI features. These imply that Prd-AD represents an intermediate state between MCI and AD.

Funding: Spanish Ministry of Health (FISS 03/0293 and FISS 02/0451)

\section{SL04:8:7}

\section{DIFFUSION TENSOR IMAGING IN AMYOTROPHIC LATERAL SCLEROSIS: QUANTITATIVE ASSESSMENT OF THE CORTICOSPINAL TRACT DEGENERATION}

\section{G. Sparacia, C. Gagliardo, R. Lagalla, M. Midiri}

University of Palermo, Dept. of Radiology, Palermo, Italy

\section{OBJECTIVE}

To determine whether diffusion-tensor imaging (DTI) indexes are valuable in detecting corticospinal tract (CST) degeneration in patients with amyotrophic lateral sclerosis (ALS).

\section{MATERIALS AND METHODS}

Twentytwo patients with ALS with definite upper motor neuron (UMN) clinical signs and 22 healthy subjects were studied by means of DTI. DTI was acquired with a 25 -direction, single-shot, spin-echo echo-planar sequence $\left(b=1000 \mathrm{sec} / \mathrm{mm}^{2}\right)$.

Mean diffusivity (MD) and fractional anisotropy (FA) were measured along the corticospinal tracts in each patient and subject. Differences in diffusion-tensor imaging indexes between patients with ALS and control subjects were evaluated with Mann-Whitney testing.

\section{RESULTS}

Mean diffusivity and FA in control subjects were respectively $701.3 \times 10(-6) \mathrm{mm}^{2} / \mathrm{sec}+/-30.1$ and $0.615+/-0.033$. MD was significantly increased $\left(784.3 \times 10(-6) \mathrm{mm}^{2} / \mathrm{sec}+/-37.2, \mathrm{P}<.05\right)$ and FA significantly decreased $(0.532+/-0.038, \mathrm{P}<.05)$ along the corticospinal tracts in patients with UMN involvement compared to control subjects.

CONCLUSIONS

Diffusion tensor imaging can detect corticospinal tract degeneration in patients with amyotrophic lateral sclerosis and is potentially useful as a quantitative tool for monitoring the progression of UMN pathology.

\section{SL04:8:8}

\section{IMAGE QUALITY, DISTORTION AND SAFETY} ISSUES IN POSTOPERATIVE IMAGING AFTER DEEP BRAIN STIMULATION.

S. Ulmer ${ }^{1}$, M. Helle ${ }^{1}$, J. Herzog ${ }^{2}$, M. Pinsker ${ }^{3}$, D. Falk ${ }^{3}$, $\overline{\text { M. Tietke }}^{1}$, H. Mehdorn ${ }^{3}$, G. Deuschl ${ }^{2}$, O. Jansen ${ }^{1}$

${ }^{1}$ Section of Neuroradiology, Department of Neurosurgery, University Hospital of Schleswig-Holstein, Kiel, Germany;

2 Department of Neurology, University Hospital of SchleswigHolstein, Kiel, Germany; ${ }^{3}$ Department of Neurosurgery, University Hospital of Schleswig-Holstein, Kiel, Germany

\section{PURPOSE}

Deep brain stimulation (DBS) of the subthalamic nucleus (STN) is performed in advanced parkison's disease for treatment of rigidity, tremor, and bradykinesia. The benefit of DBS has been demonstrated compared to medication alone, compared to sham stimulation and in the long term follow-up. The most effective stimulation site and thus the target of the stimulation electrodes is the dorsolateral margin of the subthalamic nucleus. Postoperatively patients undergo MR imaging to document the location of the electrodes. Device movement, heating or induced voltage are the safety-related issues in deep brain stimulation electrodes and MRI. There has been a FDA recommendation to lower the SAR thresholds from $0.4 \mathrm{~W} / \mathrm{kg}$ to $0.1 \mathrm{~W} / \mathrm{kg}$ in 2005.

\section{MATERIAL AND METHODS}

Implantation of deep brain stimulation electrodes (model 3389, Medtronic, Minneapolis, USA) was performed in 235 cases for parkinsons disease in our institution. Over the years different scanners and SAR limits had been used, thus we reviewed the postoperative images of three different epoches in terms of image quality and reviewed the charts in terms of perioperative complications. Furthermore a phantom was created to measure and describe the size of the artefact.

\section{RESULTS}

Using a 6-rank score image quality did not differ between scanners or SAR limits (one-way ANOVA, $\mathrm{F}=0.4, \mathrm{p}=0.67$ ). There were no MR related complications. The artefact of the electrodes was triangular arrowhead-shaped with a rounded tip pointing towards the readout direction and a broader base in the phase encoding direction resembling an isosceles triangle in approximation. The artefact was 5.3-fold the real size of the tip of the electrode.

CONCLUSION

Previously the artefact has been described as a roundish shape, however, as demonstrated here it rather resembles an isosceles triangle five-fold the real size of the tip of the electrode. This is an important finding in judging the stereotactic result and in understanding why in some patients stimulation might be less effective than in others. Reduction of the SAR was considered to result in lower image quality, however, it turned out to be sufficient enough for postoperative image interpretation. MRI can be performed safely following the manufacturers recommendations with acceptable image quality

\section{September 22, 2007 Maestrale Room}

\section{MA05}

09.50-11.20 - Tumors I 


\section{MA05:9:1}

\section{ACCURACY OF PRE-OPERATIVE NEURO-IMAGING IN BRAIN TUMOURS}

\section{Nayeemuddin, M. Majeed, T. Muthu,}

M. Christie, K. Sherlala

Walsgrave Hospital, University Hospitals Of Coventry And Warwickshire Nhs Trust, Coventry, Warwickshire, United Kingdom

\section{PURPOSE}

To compare pre-operative radiological diagnosis with the postoperative histological diagnosis in frame-based (Lekshel) computerised tomography (CT) scan guided stereotactic brain biopsy

METHODS

Retrospective analysis of case notes, theatre registers, CT scan registers and reports (by experienced neuro-radiologists) was done over a period of 3 years (2002-2005)

RESULTS

A total of 41 stereotactic biopsies were performed. Mean age was 59.34 years (range 20-87). Male to female ratio was 1.2:1. Locations of tumours were: frontal $15(37 \%)$, temporal $2(5 \%)$, parietal $6(15 \%)$, occipital $2(5 \%)$, corpus callosum $3(7 \%)$, basal ganglia $5(12 \%)$, thalamus $3(7 \%)$, pineal gland $1(2.5 \%)$, lateral ventricle $1(2.5 \%)$ and multiple cerebral ring lesions $3(7 \%)$. Histologically, 21 (51\%) were glioblastoma multiforme (GBM), 6 (15\%) were astrocytomas, 2 (5\%) were oligodendroglioma, 2 (5\%) were Non-Hodgkin's lymphoma (NHL), 1 (2.5\%) each of germinoma, ependymoma and metastasis and 7 (17\%) were inconclusive. Diagnostic yield was $83 \%$. Pre-operative radiological diagnosis by Magnetic Resonance Imaging (MRI) was available in $29(71 \%)$ out of 41 . Histological diagnosis was obtained in $36(83 \%)$ out of 41 . In $18(62 \%)$ out of 29 cases, preoperative radiological diagnosis matched exactly with the final histological result. Pre-operative radiology was consistent with histology in 12/15 (80\%) of GBM, $2 / 2(100 \%)$ of oligodendroglioma, 1/1 (100\%) metastasis, $2 / 4(50 \%)$ of astrocytoma and $1 / 2$ $(50 \%)$ of NHL and none $(0 / 5)$ of the inconclusive cases. Operative mortality ( $<30$ days) was $4.8 \%$.

\section{CONCLUSION}

Pre-operative radiological diagnosis seems to be consistent and relates well with histological diagnosis especially in cases of GBM. This may decrease the number of invasive biopsy procedures performed along with its associated mortality and morbidity.

\section{MA05:9:2}

\section{ATYPICAL PARASELLAR PILOCYTIC JUVENILE ASTROCYTOMA}

A. Rosanò, A. Bocchio, D. Machado,

E. Bottacchi, G. Giardini, T. Meloni

Ospedale Regionale Della Valle D'aosta Aosta Italy

\section{METHODS}

Young male, 14 years old,in May 2006 comes to emergency for pulsing headache,right hemiparesis and right VII cranic nerve deficit.

CT scanner: nodular left parasellar-perisylvian mass with unhomogeneous contrast enhancement

Transcranial Doppler :acceleration of blood flow in left carotid siphon and M1.

MR : multiples micronodules of pathologic tissue, appearing extraparenchimal, in left perisellar cistern and sylvian scissure, involving branches of intracranial arteries (C7, A1, M1);diffusion weighted images (DWI) reveals left ischemic capsulo-lenticular and omolateral radiate crown lesions.

Serologic tests and liquor:normal.During hospitalization progressive,spontaneous remission of sinthoms is observed.Patient is discharged with corticostheroid therapy,in suspect of inflamatorygranulomatous process involving intracranial branches of left carotid artery as cause of stroke. MR controls, in following 9 months, show slow increasing of number and volume of perisylvian lesions. Angiography (november 2006) : only minimal stretching and narrowing of A1, M1 e C7.

Surgery (february 2007): subradical resection;isthologic diagnosis of pilocytic astrocytoma (WHO I).

RISULTS

Pilocytic astrocytoma is the most common pediatric glioma. Cerebellum, optic nerve-chiasm and hypothalamic region are the most common locations.

Most optic pathway pilocytic astrocytomas (75\%) arise in children less than 12 years old;clinical symptoms and signs are usually of several months duration, directly related to the specific location of the tumor.

Classic appareance is of a cystic mass with enhancing mural nodule. Differential diagnosis of chiasmal/hipotalamic astrocytomas includes craniopharingiomas, germ cell tumors,hypothalamic gangliogliomas and granulomatous diseases such as tuberculosis and sarcoidosis.Presentation before age 5 years favors the diagnosis of astrocytoma,as does association with NF1.Clinical history can also be helpful.

CONCLUSIONS

Pilocytic juvenile astrocytoma, characterized by classic"cystic mass with enhancing mural nodule"imaging appearance, is easily recognised in most circumstances.

In our case stroke-like clinical presentation, location (parasellar), extension of lesion (beside sylvian fissure),imaging appearance (multiple small nodules, with peripherical contrast enhancement) were unusual and made the diagnosis very difficult.

\section{MA05:9:3}

\section{MR IMAGING ABNORMALITIES IN OPSOCLONUS MYOCLONUS SYNDROME}

\author{
$\underline{\text { L. Van Den Hauwe }}{ }^{1}$, K. Geens ${ }^{1}$, E. Pelzers ${ }^{1}$, \\ J. Van Goethem ${ }^{2}$, P. Parizel ${ }^{2}$ \\ 1 AZ KLINA, Brasschaat, Belgium; ${ }^{2}$ Antwerp University \\ Hospital, Antwerp, Belgium
}




\section{PURPOSE}

To report brainstem abnormalities on magnetic resonance (MR) imaging in paraneoplastic opsoclonus-myoclonus syndrome (OMS).

\section{CASE REPORT}

A 49-year-old woman was referred for MR imaging of the brain because of progressive oscillopsia, dizziness and dysphagia. FLAIR- and T2-weighted sequences demonstrated high signal intensities in the tegmentum and periaqueductal gray matter. Diffusion-weighted imaging did not display diffusion restriction. There was no enhancement of the lesions after gadolinium injection.

Anti-Ri antibodies in serum and CSF were markedly elevated, very suggestive for the presence of breast cancer. Mammography however was negative. CT of the thorax-abdomen as well as whole body FDG-PET were negative at that time. Patient's symptoms improved with corticosteroids and plasma exchange. Three years later, patient developed bilateral breast carcinoma. DISCUSSION

OMS is a rare neurological disorder, which is characterized by chaotic eye movements and myoclonus; sometimes ataxia is present. It is associated with multiple etiologies; the pathophysiology may be autoimmune. Paraneoplastic OMS has been described in children with neuroblastoma; in adults, breast cancer and small-cell lung cancer are the most common primary tumors. Antineuronal antibodies (e.g. anti-Ri) formed in response to the presence of tumor may cross-react with cells of the nervous system. Symptoms may precede the diagnosis of cancer by months or years and often have a rapidly progressive course. Opsoclonus is the result of a dyskinesia of the saccadic oculomotor system consisting of three different neurons (burst, tonic, and pause cells) all located in the pontine tegmentum, more specific in the paramedian pontine reticular formation. Only few cases with MR imaging abnormalities in the brain have been described.

CONCLUSIONS

OMS may be the first manifestation of breast cancer. MR imaging may reveal non-specific abnormalities in the pontine tegmentum as a result of cross-reaction between neuronal antibodies and specific nervous cells.

\section{MA05:9:4}

\section{DIFFUSION WEIGHTED IMAGING AND APPARENT DIFFUSION COEFFICIENT (ADC) OF ACOUSTIC NEURINOMAS AT MR-IMAGING.}

\section{F. Cartes-Zumelzu $^{1}$, K. Roessler ${ }^{2}$,}

W. Dietrich ${ }^{3}$, C. Mueller-Mang ${ }^{1}$, G. Widhalm ${ }^{3}$, E. Knosp ${ }^{3}$, M. Thurnher ${ }^{1}$

1 Medical University Vienna,Dep.Of Radiology-Division Of Neuroradiology, Vienna, Austria; ${ }^{2}$ Landeskrankenhaus Feldkirch, Feldkirch, Austria; ${ }^{3}$ Medical University Vienna,Dep.Of Neurosurgery, Vienna, Austria
BACKGROUND AND PURPOSE

Given the fact that brain tumor imaging has driven from a morphology based discipline to one that might elucidate biologic tumor alterations we undertook this study to evaluate if DWI and ADC values could provide additional useful information in the diagnosis and assessment of patients with Vestibular Neurinomas. METHODS

We studied retrospectively 36 patients with 37 acoustic nerve neurinomas.

28/37Tumors were histologically proven.9/36 patients underwent y-knife surgery.

All patients were examined between 2002 and 2007 on a 1.5 Tand

3.0 T scanner. Conventional MR Imaging and echo-planar diffusion weighted imaging (DWI) was performed in all patients. Mean patient age was $47 \mathrm{y} \pm 16(\mathrm{SD})($ range $=18-79 \mathrm{y})$.

An additional tumor-classification according to the intraoperative macroscopic tumor aspect was undertaken into compact, soft and cystic.

\section{RESULTS}

A significant difference in ADC values was seen between Tumors and normal brain tissue $(\mathrm{p}=0,01)$.

28/37 Vestibular Neurinomas showed mean ADC values of $1.33 \pm$ $0,44(\mathrm{SD}) \times 10-3 \mathrm{~mm} 2 / \mathrm{sec}($ range $=0,7-3,6 \times 10-3 \mathrm{~mm} 2 / \mathrm{sec})$. Contralateral brain parenchyma showed mean ADC values of $0.76 \pm$ 0,28(SD)x $10-3 \mathrm{~mm} 2 / \mathrm{sec}$.

Seventeen/28 tumors were classified as compact, 9/28 as soft and $2 / 28$ as cystic.

$\mathrm{ADC}$ values of macroscopically compact tumors was lowest (mean = $1,21 \pm 0,23 \times 10-3 \mathrm{~mm} 2 / \mathrm{sec})$. Mean $\mathrm{ADC}$ values of macroscopically soft tumors was highest with $1,42 \pm 0,5 \times 10-3 \mathrm{~mm} 2 / \mathrm{sec}$. Macroscopically cystic tumors showed mean ADC values of $1,36 \pm 0,46 \times 10-3 \mathrm{~mm} 2 / \mathrm{sec}$. On DWI $58,8 \%(\mathrm{n}=20 / 28)$ were isointense, $16,8 \%(n=6 / 28)$ were inhomogeneous and $5,6 \%(n=2 / 28)$ were hypointense.

\section{CONCLUSIONS}

Based on these preliminary datas, these findings are consistent with increased amounts of extracellular water allowing relatively increased motion of water molecules. Compact tumors showed lowest ADC values and soft tumors the highest. In conclusion we suggest that according to our DWI/ADC data acoustic neurinomas may represent a solid tumor group with a relatively loose tumor matrix. DWI and ADC may aid in differentiating acoustic neurinomas from other tumors in the cerebellopontine angle and may aid in elucidating pre-operatively the tumor character. 


\section{MA05:9:5}

\section{STAGING ESTHESIONEUROBLASTOMA - A NEW ANSWER} TO AN OLD QUESTION

\author{
C. Ozdoba ${ }^{1}$, G. Schroth ${ }^{1}$, L. Remonda ${ }^{1}$, A. Lukes ${ }^{2}$, \\ $\overline{\text { M. Speiser }}^{3}$, K. Laedrach ${ }^{3}$, J. Raveh ${ }^{3}$ \\ ${ }^{1}$ Institute of Diagnostic and Interventional Neuroradiology, University \\ of Bern / Inselspital, Bern, Switzerland; ${ }^{2}$ Department of Neurosurgery, \\ University of Bern / Inselspital, Bern, Switzerland; ${ }^{3}$ Department of \\ Cranio-Maxillo-Facial Surgery, University of Bern / Inselspital, Bern, \\ Switzerland
}

\section{PURPOSE}

Esthesioneuroblastoma (ENB) is a highly malignant, rather rare tumor. The currently most commonly used classification/staging system, the Kadish staging, was presented in 1976. Kadish C, the highest grade, however, only denotes the presence or absence of tumor spread beyond the paranasal sinuses without further differentiation. Numerous attempts have been made to develop a staging system for ENB that correlates with outcome and longtime-survival.

At our institution, skull-base surgeons and neuroradiologists jointly developed a classification system that is more detailed than the Kadish staging as it takes into account the detailed degree of tumor infiltration into adjacent structures.

\section{METHODS}

Between 1989 and 2004, fifteen patients (nine men, six women, age 16-77 years, mean 52.4 years) were operated for ENB at our institution. Preoperatively, all tumors had been classified as Kadish C. In the new staging system that we present here, we examined 23 different anatomic structures around the skull base for tumor infiltration; according to the infiltrated location, one to five points (reflecting, e.g., the difficulty to surgically remove tumor tissue in this location) were given. As a maximum, 78 points could be reached.

Results: High-resolution CT and MRI studies allowed the delineation of the defined anatomic landmarks in all cases. The degree of tumor infiltration varied between 25 and 68 points. The mean and median were 45.5 and 43.5 points, respectively. KaplanMeyer survival analysis performed for a cut-off at 45 points showed marked differences between the low and the high infiltration groups.

CONCLUSIONS

Detailed analysis of high resolution digital imaging allows to clearly define ENB infiltration into neighbouring anatomic structures. The suggested classification system, which is more detailed than the Kadish staging, correlates well with long-term outcome. Further studies, however, will be necessary to establish this system's value in a larger group of patients.

\section{MA05:9:6}

\section{INTRAOPERATIVE MRI AT 3T USING A NEUROSURGICAL-MR SUITE DESIGNED FOR COMBINING CLINICAL AND SURGICAL ACTIVITIES}

T. Duprez, G. Ribeiro-Vaz, A. Jankovski, M. Docquier, M. Van Boven, L. Hermoye, C. Grandin, G. Cosnard, C. Raftopoulos Université catholique de Louvain, Brussels, Belgium

\section{PURPOSE}

To assess feasibility, safety, timing, and clinical efficiency of an original intraoperative MR procedure using a clinical $3 \mathrm{~T}$ MR system adjacent to a neurosurgical operating room

\section{MATERIAL AND METHODS}

an original complex was built connecting a clinical $3 \mathrm{~T}$ MR system dedicated to clinical and research work to a neurosurgical room. Patient's translation back and forth from the operating table to the MR support was enabled through a fully motorized operating table abutting to an intermediate lock chamber opening on the MR system. Connecting home-manufactured table-guides put on both MR and operating table allowed fast, safe and shock less transfer of the patient. MR room was equipped with a laminar pulsed air cleaning system to fulfil asepsis requirements. A complex but fully automated procedure of 20 minutes duration was used for switching the MR system from clinical to surgical modality. Standard flexible surface coils wrapped into sterile drapes were used as receivers. 50 patients (average age: 47,8 years; weight $5-110 \mathrm{~kg}$ ) had intraoperative MR examination (40 intracranial neoplasms, 5 chronic refractory epilepsia, 2 tumor + epilepsia surgery). Three patients undergoing cervical spinal cord tumor resection had intraoperative MRI

RESULTS

Mean duration for completing the whole MR procedure was 83 minutes (range 58-129). No significant drawback from the procedure was observed, except slight skin burns in 2 cases. Very High quality images were obtained for all common sequences (FFE T1, FSE T2, FLAIR, DWI, EPI-T2*, MRA). Intraoperative brain MR procedure led to second surgical look in seven cases, with five resulting in successful additional resection. Precocious and intense contrast-enhancement of the margins of the resection cavity was the leading cause for false-positivity in cases being interpreted as positives for tumoral residue but having negative delayed MR follow-up examination. High quality images were obtained for cervical spine imaging including relevant DTI tractograms. Sustained research/clinical activity was uninterruptedly maintained in-between intra-operative procedures with tolerable appointment shifts for scheduled examinations $(3,000$ per year).

\section{CONCLUSION}

However time-consuming, brain or spinal intraoperative MR examination using a radiological-neurosurgical facility designed for combining clinical, research, and intraoperative activities and equipped with a $3 \mathrm{~T}$ state-of the-art system was demonstrated feasible, safe, and clinically relevant. 


\section{MA05:9:7}

DYNAMIC CONTRAST-ENHANCED SEQUENCES DURING INTRAOPERATIVE HIGH-FIELD MAGNETIC RESONANCE IMAGING TO EVALUATE THE RADICALITY OF PITUITARY MACROADENOMAS SURGERY

F. Fasoli 1, A. Romano 1, M. Ferrante 1, G. Trillo' 2, $\overline{\text { E. Ramund }}$ Orlando $^{2}$, L. Fantozzi ${ }^{1}$, A. Bozzao ${ }^{1}$

${ }^{1}$ Neuroradiology, Ii Faculty University La Sapienza, Rome, Italy;

${ }^{2}$ Neurosurgery, Ii Faculty University La Sapienza, Rome, Italy

\section{PURPOSE}

To assess the value of dynamic contrast-enhanced MR imaging with intra-operative high field strength magnetic resonance (i-MR) imaging in the treatment of pituitary adenoma and its impact on detecting residual adenoma.

\section{METHODS AND MATERIALS}

Twenty-nine patients with pituitary adenoma were studied with a 1.5 T i-MR unit. In all patients, the surgical approach has been Endonasal Mono-nostril Transsphenoidal endoscopic-controlled. MRI was performed before and during the surgical procedure with T1- and T2-weighted sequences, before and after intravenous Gd-DTPA administration; dynamic sequences during intravenous contrast injection were acquired as well. The rate of complete tumour removal was evaluated comparing images obtained in pre-operative phase. MR follow-up ranging from 1 to 3 months was performed as well. RESULTS

In 10 patients (34\%), initial iMR demonstrated that the endpoint for extent of resection had been achieved, 3 of these still had residual suprasellar tumour either in contact with the optic chiasm or adjacent to the cavernous sinus, contraindicating further surgery. In 19 patients (66\%), iMR imaging documented the presence of a residual tumour. In 13 of 19 patients, the residual tumour was removed during early surgical procedure; in the 6 remaining cases complete surgical removal was achieved after further surgical manoeuvres. When intraoperative MRI was acquired to evaluate tumour removal, only dynamic sequences (compared to $\mathrm{T} 2$ and $\mathrm{T} 1$ pre- and post-contrast) were considered capable of distinguishing the residual pathology in 12 cases.

\section{CONCLUSION}

Dynamic MRI is mandatory for detection of pituitary residual tumour during intra-operative MRI, thereby significantly increasing the rate of complete tumour removal.

\section{MA05:9:8}

\section{MR DEPICTION OF CEREBRAL GLIOMAS WITH CONTRAST-ENHANCEMENT: IMPLICATIONS FOR THERAPEUTIC INTERVENTION}

\footnotetext{
M. Kuhn ${ }^{1}$, J. Maldjian ${ }^{2}$, N. Anzalone ${ }^{3}$, P. Picozzi ${ }^{3}$

${ }^{1}$ St. Johns Hospital, Springfield, USA; ${ }^{2}$ Wake Forest Univerity, Winston-Salem, USA; ${ }^{3}$ San Raffaele Hospital, Milan, Italy
}

\section{PURPOSE}

The success of surgical resection of cerebral gliomas is highly dependent on accurate presurgical determination of tumor margins. MR contrast enhancement at standard doses of contrast media may underestimate the true extent of gliomas. We evaluated whether the high relaxivity contrast agent gadobenate dimeglumine (GdBOPTA), given at a standard dose, provides improved contrast enhancement in patients with cerebral gliomas compared to gadopentetate dimeglumine (Gd-DTPA).

\section{METHODS}

Patients $(n=47)$ with cerebral gliomas were given two complete MR examinations at $1.5 \mathrm{~T}$ separated by $2-7$ days, one performed using $0.1 \mathrm{mmol} / \mathrm{kg} \mathrm{Gd}$-BOPTA ( $2 \mathrm{~mL} / \mathrm{sec}$ ) and the other using Gd-DTPA at the same dose and rate. Acquisition parameters and post-dose acquisition time were identical for the two examinations. Three expert blinded neuroradiologists independently evaluated images for lesion border delineation, definition of disease extent, visualization of lesion internal morphology, lesion contrast enhancement and global diagnostic preference. Quantitative lesion enhancement (\% enhancement, lesion-to-brain ratio [LBR] and contrast-to-noise ratio [CNR]) was also determined. Wilcoxon signed rank test was used to evaluate differences between contrast agents, and weighted kappa (ê) statistics were used to assess reader agreement.

RESULTS

Gd-BOPTA provided superior lesion border delineation, definition of disease extent, visualization of the internal morphology of the lesions and lesion contrast enhancement as compared with Gd-DTPA ( $p<$ 0.0001 for all assessments). Quantitative enhancement was significantly greater after Gd-BOPTA ( $\%$ enhancement and LBR: $\mathrm{p}<$ 0.0001; CNR: $p<0.004)$. In some patients use of GD-BOPTA enabled detection or satellite lesions or area of tumor spread. Reader agreement ranged from $\hat{e}=0.49(63.8 \%)$ for lesion internal morphology to $\hat{\mathrm{e}}=0.68(80.9 \%)$ for definition of disease extent.

\section{CONCLUSIONS}

Gd-BOPTA significantly improves delineation of cerebral gliomas, and provides better definition of tumor extent and internal morphology as compared to Gd-DTPA. The significantly improved depiction of gliomas achievable with $0.1 \mathrm{mmol} / \mathrm{kg} \mathrm{Gd}$ BOPTA may help optimize radiotherapy target volumes or surgical margins.

\section{MA05:9:9}

\section{CLINICAL EVALUATION OF GADOLINIUM CONTRAST MEDIA FOR ASSESSMENT OF INTRA-AXIAL BRAIN METASTASES}

\author{
M. Kuhn $^{1}{ }^{1}$, P. Picozzi ${ }^{2}$, K. Maravilla ${ }^{3}$, N. Anzalone ${ }^{2}$ \\ ${ }^{1}$ St. Johns Hospital, Springfield, USA; ${ }^{2}$ San Raffaele Hospital, \\ Milan, Italy; ${ }^{3}$ University of Washington, Seattle, USA
}

\section{PURPOSE}

Treatment of patients with brain metastases is based on accurate assessment of the number, size, and location of enhancing lesions, each of which are determined by application of a gadolinium-based contrast agent. We performed a prospective study to compare 
gadopentetate dimeglumine with the high relaxivity agent gadobenate dimeglumine for enhancement and conspicuity of intra-axial metastases.

\section{METHODS}

Patients with confirmed intra-axial brain metastases underwent two complete MR examinations at $1.5 \mathrm{~T}$, one performed with $0.1 \mathrm{mmol} / \mathrm{kg}$ bodyweight gadobenate dimeglumine and the other with an identical dose of gadopentetate dimeglumine. Contrast agents were given at $2 \mathrm{~mL} / \mathrm{sec}$ in randomized order with an interval between examinations of 2-7 days. Acquisition parameters and post-dose acquisition times were identical. Three expert readers evaluated images in a blinded manner for lesion border delineation, definition of disease extent, visualization of lesion internal morphology, lesion contrast enhancement and global preference. Quantitative lesion enhancement ( $\%$ enhancement, lesion-to-brain ratio [LBR] and contrast-to-noise ratio [CNR]) was determined.

RESULTS

Thirty seven patients were included in this study. Quantitative enhancement was significantly greater after gadobenate dimeglumine ( $\%$ enhancement: $\mathrm{p}<0.013$; LBR: $\mathrm{p}<0.002$; CNR: $\mathrm{p}<0.04)$. Contrast enhancement of metastatic lesions was preferred in $49 \%, 57 \%$, and $68 \%$ of subjects after gadobenate dimeglumine compared with $5 \%$, $8 \%$, and $10 \%$ of subjects after gadopentetate dimeglumine (readers 1 , 2, 3, respectively). Similar improvements were noted for global preference and for all other qualitative parameters. Reader agreement was good for all evaluations (up to $\hat{\mathrm{e}}=0.55 ; 67.6 \%$ ).

\section{CONCLUSIONS}

In blinded quantitative and qualitative assessments, enhancement and visualization of brain metastases was significantly improved with use of the high relaxivity contrast agent gadobenate dimeglumine. For patients with CNS metastatic disease, better contrast enhancement may translate into improved treatment decision-making based on a more accurate assessment of the number, size, and location of metastatic lesions.

\section{MA05:9:10}

\section{MULTICENTRE CROSSOVER COMPARISON \\ OF GADOBENATE DIMEGLUMINE AND GADODIAMIDE FOR ENHANCED MR IMAGING OF CNS LESIONS}

H. Rowley ${ }^{1}$, G. Scialfa ${ }^{2}$, P. Gao ${ }^{3}$, J. Maldjian ${ }^{4}$, D. Hassell ${ }^{5}$, M. Kuhn ${ }^{6}$, M. Gallucci ${ }^{7}$, J. Ruscalleda ${ }^{8}$, S. Bastianello ${ }^{9}$, C. Colosimo ${ }^{10}$

${ }^{1}$ University of Wisconsin, Madison, WI, USA; ${ }^{2}$ Hospital Niguarda Ca'Granda, Milan, Italy; ${ }^{3}$ Beijing Tianan Hospital, Beijing, China;

${ }^{4}$ Wake Forest University, Winston-Salem, NC, USA; ${ }^{5}$ Providence Hospital, Mobile, AL, USA; ${ }^{6}$ Southern Illinois University, Springfield, IL, USA;

${ }^{7}$ Universita de L'Aquila, L'Aquila, Italy; ${ }^{8}$ Hospital Sant Pau, Barcelona, Spain; ${ }^{9}$ University of Pavia, Pavia, Italy;

${ }^{10}$ Catholic University of the Sacred Heart, Rome, Italy

\section{PURPOSE}

Previous studies have suggested that gadobenate dimeglumine provides better enhancement than the widely used agent gadopentetate dimeglumine. We performed a large scale multiinstitutional trial to evaluate whether improved enhancement was seen when comparing gadobenate dimeglumine with gadodiamide in patients with CNS lesions.

\section{METHODS}

Adult patients with suspected or known brain lesions underwent two identical MRI exams at $1.5 \mathrm{~T}$, one with gadobenate dimeglumine (MultiHance) and the other with gadodiamide (Omniscan). The agents were administered in randomised order at $0.1 \mathrm{mmol} / \mathrm{kg}$ bodyweight, with the two MRI exams separated by 3-14 days. Imaging sequences and post-injection acquisition timing were identical for the two exams (T1wSE and highresolution T1wGRE sequences at 3-7 min postdose). Images were evaluated in matched pairs in a blinded manner by 3 expert readers for diagnostic information (lesion border delineation, definition of disease extent, visualisation of lesion internal morphology, lesion contrast enhancement, global diagnostic preference) and quantitative enhancement (lesion-to-brain ratio [LBR], contrast-to-noise ratio [CNR]). The Wilcoxon signed rank test was used to test for differences between the two groups, and (kappa [K] statistics) for inter-reader agreement were determined.

RESULTS

No subject with impaired renal function was enrolled in the trial. Of the 113 evaluable subjects $46(41 \%)$ had intra-axial brain tumours, $29(26 \%)$ had metastatic tumours, 19 (17\%) had extraaxial brain tumours, and $19(17 \%)$ had other diagnoses. On postdose T1wSE and T1wGRE sequences, LBR and CNR were significantly higher $(\mathrm{p}<0.0001)$ after gadobenate dimeglumine (LBR increase of $0.24-0.25$ across readers, CNR increase of 9.616.1 across readers). The 3 readers demonstrated a global diagnostic preference for gadobenate dimeglumine in $63(56 \%)$, $77(68 \%)$ and $73(65 \%)$ patients, respectively, compared with 3, 2 and 3 patients for gadodiamide $(\mathrm{p}<0.0001$; all readers). Similarly highly significant preferences for Gd-BOPTA were demonstrated for all individual diagnostic information and quantitative enhancement endpoints $(\mathrm{p}<0.0001$; all readers, all comparisons). Interreader agreement was good for all evaluations $(\kappa=0.47-0.69)$.

\section{CONCLUSIONS}

Gadobenate dimeglumine significantly improves available diagnostic information and brain lesion enhancement compared to gadodiamide at a dose of $0.1 \mathrm{mmol} / \mathrm{kg}$ bodyweight. The superior enhancement provided by gadobenate dimeglumine in patients with brain tumours improves lesion characterization and treatment planning, and may avoid the need for higher contrast doses in patients with poorly enhancing lesions. 
MA05:9:11

\section{LABELING GLIOMA CELLS WITH GADOFLUORINE M PROVIDES GOOD T1-CONTRAST WITHOUT IMPEDING CELL FUNCTION.}

$\underline{\text { M. Brockmann }}^{1}$, I. Nolte ${ }^{1}$, B. Misselwitz ${ }^{2}$, C. Groden $^{1}$

${ }^{1}$ Department od Neurradiology, University Hospital Mannheim, Mannheim, Germany; ${ }^{2}$ Bayer Schering Pharma AG, CRBA Diagnostic Imaging, Berlin, Germany

MRI has become an increasingly popular technique to study migration and distribution of different cells in vivo. For this purpose one possibility is to label cells in vitro prior to injection in a target tissue. Gadofluorine is a novel macrocyclic, amphiphilic gadolinium-based contrast agent. Labeling of malignant glioma cells in vitro using Gadofluorine was feasible without the need for transfection agents or other additional means.

Labeling with Gadofluorine enhanced visualisation of glioma cells in T1-weighted sequences, even after culturing cells in medium without Gadofluorine. Migration and proliferation of glioma cells was not impeded by labeling with Gadofluorine. Significant loss of Gadofluorine into cell culture medium was ruled out by MRI. Confocal laser fluorescence microscopy revealed Cy-5-labeled Gadofluorine to be localized in the perinuclear cytoplascmic region, but not within the nucleus nor bound to cell membrane.

We conclude that Gadofluorine can easily be used to label glioma cells in vitro without affecting glioma cell biology, which is an important issue in molecular imaging. Gadofluorine provides an interesting alternative for cellular labelling, if iron oxide particles are incorporated insufficiently by target cells or if the vicinity of susceptibility artefacts prohibits the use of signal-decreasing contrast agents.

\section{MA05:9:12}

\section{MR IMAGING OF THE CNS: IS THERE A ROLE FOR CONTRAST AGENTS WITH HIGH RELAXIVITY?}

\section{Essig ${ }^{1}$}

German Cancer Research Center, Heidelberg, Germany

\section{PURPOSE}

To ascertain if the high in vivo $\mathrm{T} 1$ relaxivity of GadobenatDimeglumin (Gd-BOPTA) $(9.7 \mathrm{mM}-1 \mathrm{~s}-1)$ is clinically beneficial for MRI of CNS disease, several clinical studies were conducted to evaluate Gd-BOPTA at doses up to $0.3 \mathrm{mmol} / \mathrm{kg}$

\section{MATERIALS AND METHODS}

In a first step Gd-BOPTA at doses up to $0.2 \mathrm{mmol} / \mathrm{kg}$ was compared with standard agents at doses up to $0.3 \mathrm{mmol} / \mathrm{kg}$ for MRI of patients with CNS metastases.

The effects of Gd-BOPTA on lesion detection, enhancement and diagnosis are discussed in terms of the enhancement mechanism of
Gd-BOPTA, which involves weak and transient interaction with serum albumin.

In a second step blinded randomized intraindividual comparative studies were realized in patients with cerebral gliomas and metastases at a standard dose of $0.1 \mathrm{mmol} / \mathrm{kg}$. For a quantitative analysis tumor contrast and contrast-to-noise ratios were determined out of ROIs in tumor, unaffected white matter, a region outside the head, and an external reference tube. For the qualitative assessment on- and offsite readers were asked to compare both MR scans for lesion contrast, lesion delineation and information upon the internal morphology and structure.

\section{RESULTS}

In patients with metastases a dose of $0.1 \mathrm{mmol} / \mathrm{kg}$ Gd-BOPTA is sufficient for most clinical situations while a cumulative $0.2 \mathrm{mmol} / \mathrm{kg}$ dose provides additional information in certain cases. A cumulative $0.3 \mathrm{mmol} / \mathrm{kg}$ dose does not provide additional information beyond that available with a $0.2 \mathrm{mmol} / \mathrm{kg}$ dose. Comparative studies revealed that $0.1 \mathrm{mmol} / \mathrm{kg}$ Gd-BOPTA offers improved performance compared to comparator agents at equal dose and that $0.2 \mathrm{mmol} / \mathrm{kg}$ Gd-BOPTA is equivalent to a cumulative $0.3 \mathrm{mmol} / \mathrm{kg}$ dose of comparator.

In the intraindividual comparative studies there was a substantial better lesion delineation. Additionally, Gd-BOPTA proved to be superior to standard compounds in the qualitative analysis. When questioned if they preferred the first or the second CM the onsite investigators named Gd-BOPTA in the majority of cases $(\mathrm{p}<0.01)$. The subjective assessments were confirmed by the objective measurements of signal intensity which showed significant higher signal intensity and contrast values for Gd-BOPTA $(p<0.01)$.

\section{DISCUSSION}

Gd-BOPTA is a safe and valuable contrast media for the assessment of CNS neoplasms. Gd-BOPTA proved to be clearly superior to Gd-DTPA in the contrasting of MR images of CNS tumors. The superiority of Gd-BOPTA was demonstrated with statistical significance in the qualitative subjective assessments as well as in the objective signal intensity measurements.

\section{MA06}

11.45-12.30 - The aging brain and neurodegenerative disorders II

\section{MA06:10:1}

VOXEL BASED MORPHOMETRY (VBM)

AND DIFFUSION TENSOR IMAGING (DTI) OF SPINO-CEREBELLAR ATAXIAS (SCA) TYPE

1 AND 2

M. Grisoli $^{1}$, M. Mandelli ${ }^{1}$, C. Mariotti ${ }^{2}$, T. De Simone ${ }^{1}$, R. Fancellu ${ }^{2}$, M. Savoiardo ${ }^{1}$, L. Minati ${ }^{1}$, M. Bruzzone ${ }^{1}$

1 Neuroradiology-Fondazione Irces Istituto Neurologico Besta, Milano, Italy; ${ }^{2}$ Neurology-Fondazione Irccs Istituto Neurologico Besta, Milano, Italy 


\section{PURPOSE}

SCAs are a group of neurodegenerative diseases caused by an expansion of CAG triplet in the coding region of the disease gene, which results in production of a mutant protein with a longer than normal polyglutamine stretch.

Structural magnetic resonance imaging (MRI) does not enable reliable differentiation of SCA1 and SCA2; imaging may be normal during the first years after onset of symptoms.

We applied VBM, Apparent Diffusion Coefficient (ADC) and Fractional Anisotropy (FA) in SCA1 and SCA2 patients to determine whether they might enable differentiation between the two types.

METHODS

Fourteen patients carrying SCA1 mutation, eleven patients with SCA2 and nine controls were studied and the data were analyzed according to optimised VBM; ADC and FA were evaluated by means of regions of interest, positioned in the cortico-spinal tract (CST) at the level of the cerebral peduncle and of the pons, in the transverse pontine fibers (TPF), in the superior (SCP) and middle cerebellar peduncles (MCP) and in the hemispheric cerebellar white matter (CWM).

\section{RESULTS AND CONCLUSIONS}

The white matter analysis, using VBM, showed a significant decrease at symmetrical CWM and MCP in SCA1 patients and at $\mathrm{MCP}$ and at pons in SCA2 patients. With respect to controls, the ADC was significantly elevated in the MCP and in CWM in SCA1, whereas in SCA2 it was elevated also in SCP, TPF and CST.

ADC was highest in SCA2. The FA was significantly lower in SCA2 than in SCA1 in the TPF and in the CST at the level of the cerebral peduncle.

Although the usefulness of DTI in differentiating SCA1 and SCA2 patients and controls is

limited due to the substantial overlap among the three groups, the finding of significant

differences and correlations with clinical scores suggests it might have an important role in characterizing the phenotypes, and providing a measurement of disease severity.

\section{MA06:10:2}

\section{ABNORMAL CEREBELLAR FIBER CONNECTIVITY IN EXTRAPYRAMIDAL MOVEMENT DISORDERS DEMONSTRATED BY DIFFUSION TENSOR IMAGING}

\author{
G. Kasprian, P. Brugger, R. Seidl, D. Haubenberger, \\ E. Auff, D. Prayer \\ Medical University of Vienna, Vienna, Austria
}

\section{PURPOSE}

To demonstrate patterns of abnormal infratentorial fiber connectivity in pontocerebellar dysplasia type 2 ( $\mathrm{PCH} 2)$, multisystem atrophy (MSA) and progressive supranuclear palsy (PSP).

\section{METHODS}

Two girls (patient 1, aged 6 months and patient 2, aged 4.5 years) suffering from a choreoatetotic/dystonic movement disorder, two male adults with the clinical diagnosis of MSA-C (patient 3), and PSP (patient 4), and 3 healthy age matched controls were examined at $1.5 \mathrm{~T}$. Additionally to a routine protocol, a diffusion tensor sequence (32 directions, slice thickness $5 \mathrm{~mm}$, field of view $230 \mathrm{~mm}$, maximum $b$ value of 1000) was acquired. On a Philips Intera release 11 workstation multi region of interest and single fiber analyses were performed (minimum FA: 0.15, maximum angle change: 27, minimum fiber length: $2 \mathrm{~mm}$ ) to demonstrate the corticospinal tract (CST) and fiber tacts passing through the superior (SCP), middle (MCP) and inferior cerebellar peduncles (ICP).

\section{RESULTS}

In patients 1 and $2(\mathrm{PCH} 2)$ characteristic features of $\mathrm{PCH} 2$ on conventional MR sequences were visible. Tractography showed a normal course of the CST, with reduced/missing fiber input to the cerebellum (Figures A,B). Pontocerebellar fibers could not be visualized in the thinned MCP. Cerebellar fiber connectivity was limited to olivo/spinocerebellar fibers in patient 1 (Figures A,B) and fibers passing through the SCP in patient 2 .

In patient 3 (MSA) the unchanged size of MCP and the missing "cross sign" on conventional MR sequences did not parallel the markedly reduced number of pontocerebellar fibers (Figure D) compared to a normal control (Figure C). The SPC and ICP fibers appeared unremarkable. Patient 4 (PSP) showed a normal morphology of ICP and MCP, but the number of the SCP fibers was minimized.

\section{CONCLUSIONS}

DTI and fiber tracking reveal characteristic but overlapping patterns of abnormal infratentorial fiber connectivity in patients with extrapyramidal movement disorders. Abnormalities of afferent and efferent cerebellar fibers can be depicted even before structural changes may appear on conventional MR sequences.
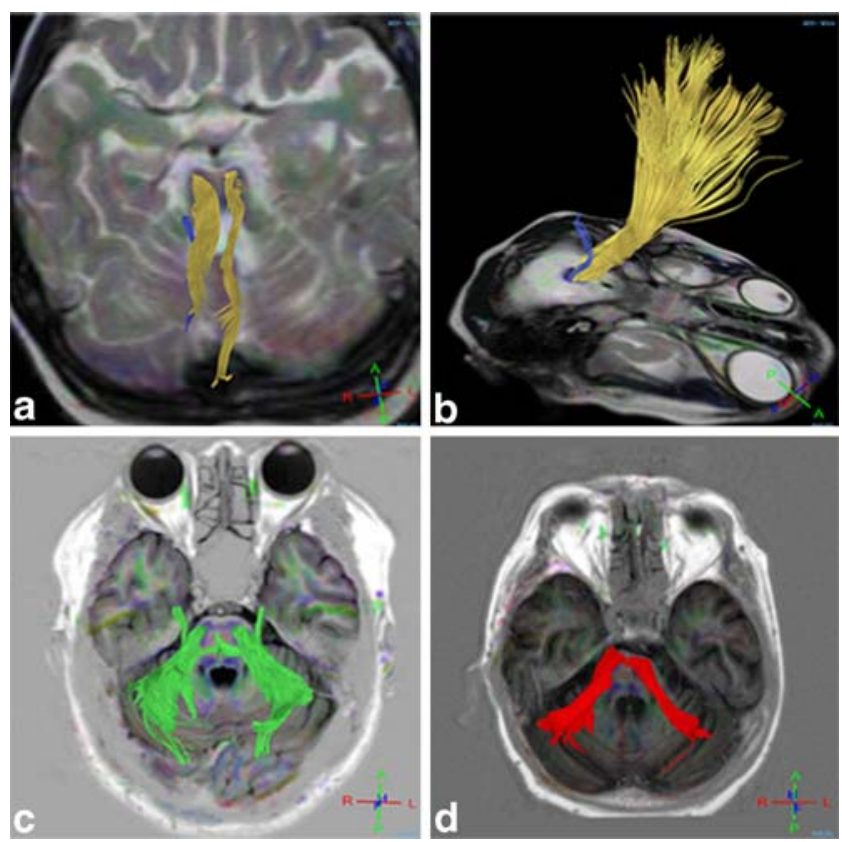


\section{MA06:10:3}

\section{BRAIN CHANGES IN DYSEQUILIBRIUM SYNDROME}

R. Raininko, A. Melberg

Uppsala university, Uppsala, Sweden

\section{PURPOSE}

Dysequilibrium syndrome is a nonprogressive neurological condition dominated by incapability of or difficulty in maintaining an upright position and in experiencing the position of the body in space i.e. the lack of sense of equilibrium. The disease is autosomal recessively inherited. Radiological studies on this disease have not been published. We performed clinical and radiological examinations in two patients without consanguinity.

METHODS

The patients were men, aged 38 and 46 years. They had nonprogressive ataxia and mental retardation. They neither had cataracts nor progressive muscle weakness. Both patients underwent brain MRI.

RESULTS

The older patient, 46 years old, had a severe volume loss/lack in the upper half of the cerebellum. The cortex was very thin and showed a high signal intensity on T2-weighted images. Very mild volume reduction was seen in the lower half of the cerebellum. The parietal sulci were widened as were also some frontal sulci.

The younger patient, 38 years old, had a very small cerebellum. The entire cerebellar cortex was thin and the cortical T2 signal intensity was high in the upper half. The pons was small in all dimensions and some volume reduction was seen in the mesencephalon and upper medulla oblongata.

The younger patient with more severe cerebellar and brain stem changes had more severe clinical symptoms.

CONCLUSIONS

Cerebellar atrophy/hypoplasia combined with a thin cerebellar cortex and a high cortical T2 signal intensity in the upper cerebellum may be a characteristic finding in dysequilibrium syndrome.

\section{MA06:10:4}

\section{PATHOGENESIS OF LEUKOARAÏOSIS IN MILD COGNITIVE IMPAIRMENT}

\section{Henry Feugeas, E. Schouman Claeys}

Bichat Claude Bernard Aphp, Paris, France

\section{PURPOSE}

Understanding the pathogenesis of leukoaraïosis (LA) appears critical to reduce rates of cognitive decline. Recent data suggest that the various subtypes of LA may all result from an imbalance between intracranial vascular pulsations amplitude and craniospinal compliance. The aim of this study was then to research altered intracranial dynamics in the elderly with mild cognitive impairment (MCI) and LA.

\section{METHODS}

71 community-dwelling older subjects with MCI underwent both structural and dynamic MR evaluations. Intracranial dynamic parameters and potential confounders including age, gender and vascular risk factors were compared between two groups respectively with and without significant LA.

\section{RESULTS}

LA was associated to ageing, reduction in total cerebral blood flow, cerebral deep venous outflow and craniospinal compliance, increase in the index of arterial pulsatility (IP) and CSF aqueductal stroke volume $(\mathrm{P}<0.03)$. In the stepwise multivariate regression, the only significant alteration of vascular "conduct function" was reduced deep venous outflow. The adjusted odds ratios for the presence of LA were respectively $9(95 \%$ CI $1-64, \mathrm{P}=0.02)$ in case of good craniospinal compliance but high IP, 10 (95\% CI 164, $\mathrm{P}=0.02)$ in case of low craniospinal compliance and high IP and $19(95 \%$ CI $3-127, \mathrm{P}=0.002)$ in case of low craniospinal compliance without high IP.

\section{CONCLUSIONS}

These results suggest that besides the known association between atherosclerosis and LA, there may be an underestimated role of inefficient craniospinal compliance in LA and cognitive decline

\section{MA06:10:5}

\section{MOBILITY DYSFUNCTION IN MILD COGNITIVE IMPAIRMENT: A MRI-BASED CLINICAL DEFINITION}

\section{Henry Feugeas, E. Schouman Claeys Bichat Claude Bernard Aphp, Paris, France}

\section{PURPOSE}

Changes in gait and balance of unknown origin predict dementia in the elderly. Periventricular leukoaraïosis (LA) has been associated to both mobility dysfunction and cognitive decline in mild cognitive impairment (MCI). But LA pathogenesis is also controversial in MCI, thus pathogenesis of gait disturbances in MCI remains debated. Better knowledge of clinical features of this LA-related mobility dysfunction may however help to determine the origin of both periventricular LA and mobility dysfunction in MCI. Thus, the aim of this study was to research which gait and balance parameters best correlate with periventricular LA in the elderly with MCI.

\section{METHODS}

61 ambulatory, community-dwelling elderly with MCI but no mobility complaint nor evident cause of mobility dysfunction, were included in this prospective study. They underwent both detailed geriatric evaluation and cerebral MRI.

\section{RESULTS}

There was an association between marked periventricular LA and worse scores on both timed gait speed test, "walking while talking" test, balance tests and timed "up and go" (TUG) test $(\mathrm{P}<$ $0.05)$. There was also a tendency to more frequent history of falls $(\mathrm{P}=0.06)$. After adjusting for age and ventriculomegaly on a logistic multiple regression model, performance on the TUG test 
was the only clinical predictor of periventricular LA ( $>10$ seconds, $\mathrm{P}=0.0003)$.

\section{CONCLUSIONS}

Clinical profile of MRI-defined mobility dysfunction suggests an early stage of "vascular parkinsonism" in MCI.

\section{MA06:10:6}

\section{INCREASED INTERMAMILLARY DISTANCE IN PATIENTS} WITH HYDROCEPHALUS AND ABNORMAL CONFIGURATION OF THE FLOOR OF THE III VENTRICLE AT ENDOSCOPIC VENTRICULOSTOMY

\author{
$\underline{\text { E. Tedeschi }}{ }^{1}$, C. Iaccarino ${ }^{2}$, E. Covelli ${ }^{1}$, G. Posillico ${ }^{1}$, \\ M. Belfiore $^{1}$, A. Bellotti ${ }^{2}$, G. Belfiore ${ }^{1}$ \\ ${ }^{1}$ Diagnostic Imaging Dept. - AORN S.Anna e S.Sebastiano, Caserta, \\ Italy; ${ }^{2}$ Neurosurgery Unit - AORN S.Anna e S.Sebastiano, Caserta, \\ Italy
}

\section{PURPOSE}

To determine the normal value of the intermamillary distance (IMD) in healthy subjects, and to assess, in hydrocephalic patients, a possible correlation between IMD and the anatomic configuration of the third ventricle floor (TVF).

\section{METHODS}

We reviewed the "routine" axial T1/T2-weighted 5 mm-thick 1.5 T MR slices of 100 normal subjects with no intracranial pathology, and of 20 patients with hydrocephalus of different etiology, treated with endoscopic third ventriculostomy (ETV). The endoscopic records were also reviewed for definition of anatomic TVF anomalies affecting the procedure.

\section{RESULTS}

The mamillary bodies (MB) were assessable in 89 subjects (age range: $4-85$ years) and showed no measurable distance in 74 cases $(85 \%)$, with mild splitting $(1.6 \pm 1.1 \mathrm{~mm})$ only in 13 cases with age-related brain atrophy.

IMD values, assessable in 19/20 hydrocephalic patients, were highly variable $(0.0-14.3 \mathrm{~mm})$. The $10 \mathrm{ETV}$ procedures hampered by abnormal configuration of the TVF (increased thickening or downward ballooning phenomenon) were associated with increased IMD values $(5.8 \pm 4.1 \mathrm{~mm})$, whereas the $\mathrm{MB}$ were clearly less separated $(2.1 \pm 1.7 \mathrm{~mm})$ in the 9 patients with normal TVF thickness. In one case a large suprasellar arachnoid cyst prevented visualization of MB at MRI and ETV. In the remaining 19 cases, the MRI-measured IMD values matched the endoscopic MB appearance. No clear correlation was found between IMD distance and disease duration: $5 / 8$ of patients with acute hydrocephalus showed no significant MB splitting (IMD $<2.0 \mathrm{~mm}$ ), whereas 9/12 of those with chronic hydrocephalus had IMD $>2.0 \mathrm{~mm}$.

CONCLUSIONS

In healthy subjects, the normal MB configuration is the absence of measurable IMD, with mild, atrophy-related splitting. In hydrocephalic patients, anatomic TVF anomalies influencing the ETV procedure were correlated with increased IMD at preoperative routine MRI. Preoperative IMD may serve as an indicator of anatomic anomalies of the TVF influencing the ETV procedure.

\section{MA06:10:7}

\section{MR CHARACTERIZATION OF PREMUTATED CARRIERS OF THE FRAGILE X SYNDROME: A VBM AND 1H-MRS STUDY}

B. Gomez-Anson ${ }^{1}$, G. Monte ${ }^{2}$, R. Rotger ${ }^{1}$, L. Rodriguez-Revenga ${ }^{3.4}$, M. Mila ${ }^{3,4}, \mathrm{~S}$. Capurro ${ }^{5}$

${ }^{1}$ Hospital Santa Creu I Sant Pau, Barcelona, Spain;

${ }^{2}$ Fundacio Clinic, Barcelona, Spain; ${ }^{3}$ Biochemistry And Molecular Genetics, Hospital Clinic, Barcelona, Spain;

${ }^{4}$ Idibaps, Barcelona, Spain; ${ }^{5}$ Cdi, Hospital Clinic, Barcelona, Spain

\section{INTRODUCTION}

$\mathrm{X}$-fragile syndrome is the most common cause of mental retardation, being caused by an expansion of the CGG triplet in the FMR1 gene. Recently, a "Fragile X-associated tremor/ataxia syndrome (FXTAS)" has been described among FMR1-premutated carriers (Pre-FX), and constitutes a significant number of late-onset hereditary ataxias. MR features in the brain of Pre-FX have been scarcely studied.

Objectives: To present the findings on conventional MRI, as well as the biochemical (1H-MRS) and structural MRI features in the brain of Pre-FX, compared to controls.

\section{MATERIAL AND METHODS}

14 premutated (7 males, aged 35-69), and 10 aged and sex-matched controls were included. Specific MR findings, such as described in FXTAS, were searched for on conventional MRI. 1H-MRS (SVS, PRESS, TR=1500; TE $=30 ; 8 \mathrm{NEX)} \mathrm{in} \mathrm{the} \mathrm{right} \mathrm{middle} \mathrm{cerebellar}$ peduncle, and 3D-IR SPGR axial MRI (Min TE, TI $=450$, flip angle $=$ 15, Bandwidth 15.63, FOV $=25 \times 20$, Thickness $=1.5,128$ locations, Matrix $=256 \times 192,1$ NEX) were obtained. Absolute metabolite concentration determinations (LC-Model), and Voxel Based Morphometry (VBM) analysis were performed. Statistics included t-test, ANCOVA (age and sex as covariates), and post-hoc analysis (significance at $\mathrm{p}<$ $0.05)$.

\section{RESULTS}

Pre-FX with FXTAS had more prominent typical features on conventional MRI than asymptomatic ones. There were typical 1H-MRS features in Pre-FX compared to controls: increased Creatine, and decreased NAA, Inositol and Glutamate ratios to Creatine. On VBM analysis, Pre-FX had, compared to controls, grey matter density loss predominantly in the dorso-lateral prefrontal cortex, and white matter density loss in the middle cerebellar peduncles and peritrigonal regions. CONCLUSIONS

Pre-FX show characteristic findings on conventional MRI, $1 \mathrm{H}-$ MRS and structural MRI (VBM analysis), different from controls. These agree with a developmental disorder, but its functional significance remains unknown.

Funding: Spanish Ministry of Science (I+D 04) and Health (FISS 02/0451) 
MA07

15.30-16.40 - Session XI - Brain-stenting procedures

\section{MA07:11:1}

\section{EXTRACRANIAL UNPROTECTED \\ SELF-EXPANDABLE PRIMARY \\ CAROTID-STENTING: A RETROSPECTIVE \\ ANALYSIS (2000-2006) WITH A LESS INVASIVE PERCUTANEOUS PROCEDURE}

\author{
M. Leonardi ${ }^{1}$, P. Cenni ${ }^{1}$, M. Dall'olio ${ }^{1}$, L. Raffi ${ }^{1}$, \\ L. Simonetti $^{2}$ \\ ${ }^{1}$ Neuroradiology Department, Bellaria Hospital, Bologna, Italy; ${ }^{2}$ \\ Neuroradiology, Maggiore Hospital, Bologna, Italy
}

Endovascular stenting is a consolidated alternative to thrombendarterectomy in the treatment of extracranial carotid artery atheromasic stenosis.

The most common complication of stenting is a distal embolism causing clinically silent or symptomatic cerebral ischaemia. To prevent this complication distal embolism protection devices are often used but their effectiveness remains unsettled. In addition, there is some evidence that distal embolism may actually be triggered by the protection systems due to clot formation at their distal surface or in the intimal lesions these systems cause. Another rarer complication is hyperperfusion syndrome arising during both stenting and thrombendarterectomy but more common in endovascular procedures. To avoid these complications the Neuroradiology Service at Bellaria Hospital (Bologna Local Health Trust) has devised a mini-invasive carotid stenting technique that does not require either distal embolism protection or angioplasty. The technique uses only the radial force exerted by the self-expanding stent to widen the atherosclerotic stenosis slowly and gradually. The goal of treatment has also changed from a prompt restoration of the atheromasic vessel's original calibre to slow transformation of the haemodynamic significance of the stenosis. The technique's success lies mainly in selecting the stenosis to treat using CT angiography to analyse plaque morphology and structure. We used the technique to treat 49 stenotic lesions in 46 patients. The study aims to describe and discuss our experience.

\section{MA07:11:2}

\section{MONITORING OF CLOPIDOGREL RELATED \\ PLATELET INHIBITION: CORRELATION OF NON-RESPONSE WITH CLINICAL OUTCOME IN SUPRAAORTIC STENTING}

\author{
S. Mueller-Schunk ${ }^{1}$, J. Linn ${ }^{1}$, N. Peters ${ }^{2}$, M. Spannagl ${ }^{3}$, \\ M. Dichgans ${ }^{2}$, H. Brückmann ${ }^{1}$, T. Mayer ${ }^{1}$ \\ ${ }^{1}$ Dept. of Neuroradiology, LMU, Munich, Germany; \\ 2 Dept. of Neurology, LMU, Munich, Germany; ${ }^{3}$ Dept. of \\ Hemostaseology, LMU, Munich, Germany
}

\section{PURPOSE}

The purpose of the study was to investigate the feasibility of onsite testing of clopidogrel effects in endovascular stenting and to determine the effects of standard anti platelet medication in patients undergoing neurovascular interventions. Furthermore the correlation of test results with clinical outcome and complication rates was analyzed.

\section{METHODS}

Clopidogrel effects were tested by impedance aggregometry. Two control groups were tested for reference values: 50 healthy blood donors without clopidogrel medication and 50 cardiologic patients under clopidogrel medication. 50 consecutive patients who were scheduled for neurointerventional stenting procedures were analyzed prospectively with point-of-care testing. The patients all had symptomatic arteriosclerotic lesions: 16 intracranial stenosis and 34 extracranial internal carotid artery (ICA) stenosis.

\section{RESULTS}

Reference values for responders and non-responders were established from results of the healthy control group. 9/50 (18\%) of cardiologic and 14/50 (28\%) of neurological patients were nonresponders. Adverse events were registered in 5/50 (10\%) patients, one of them with a permanent neurologic deficit (1/50,2\%). All five patients with adverse events were clopidogrel non responders. There was a statistically significant correlation between adverse events and clopiodgrel non-response (Fisher's exact test, $\mathrm{p}=0.001$ ).

\section{CONCLUSION}

On-site testing of platelet inhibition by clopidogrel with impedance aggregometry is feasible. It detects significant rates of nonresponders in a cardiologic control group and a group of neurologic patients. Our data strongly suggests a correlation of insufficient clopidogrel related platelet inhibition with an increased risk of thrombembolic events in supraaortic stenting.

\section{MA07:11:3}

\section{PRELIMINARY RESULTS OF INTRASTENT}

W. Kurre et al. ${ }^{1}$, J. Berkefeld ${ }^{2}$, D. Mucha ${ }^{2}$, J. Buhk $^{2}$, J. Maskova ${ }^{2}$, B. Eckert ${ }^{2}$, B. Turowski ${ }^{2}$, G. Trenkler ${ }^{2}$, W. Kueker ${ }^{2}$, V. Katsaridis ${ }^{2}$, G. Klein ${ }^{2}$, O. Wittkugel ${ }^{2}$

${ }^{1}$ Institut of Neuroradiology, University of Frankfurt, Frankfurt, Germany; ${ }^{2}$ Intrastent Study Group

\section{PURPOSE}

Intracranial stenting of high grade atherosclerotic stenoses is increasingly used in symptomatic patients. However, clinical effectiveness of endovascular versus medical treatment is not yet validated in comparative trials. INTRASTENT, a multicentric registry for stent treatment of intracranial stenoses was established in 2006 as a precursor for a randomized trial with the aim to create a database large enough to define proper techniques, materials and patient selection criteria. After one year we present the first interim results. 


\section{METHODS}

Eleven centres in Europe agreed to participate in INTRASTENT and included clinical, technical and follow-up data of patients with symptomatic stenoses $>50 \%$ treated with either balloon expandable or self expanding stents.

Main endpoints of the current evaluation were stroke, death and restenosis during the acute phase and after 6 months.

\section{RESULTS}

Intrastent contains data sets of 94 patients ( 77 male, 17 female). Cumulative stroke and death rate up to 30 days after the procedure was $7.2 \%$ due to three deaths, three major strokes and one minor stroke. Guidewire perforation, vessel perforation during predilatation and reperfusion hemorrhage caused procedure related deaths. Perforator occlusions were responsible for two major strokes and one minor stroke event. One major stroke syndrome was due to a stent thrombosis one week after the procedure. For 6 months follow-up 44 data sets were available and proved three TIAs and one stroke in the treated vascular territory. The only stroke and one TIA were associated with a restenosis. One TIA was due to vasculitis and one to progressive atherosclerosis. Four severe restenoses $>70 \%(18.2 \%)$ were detected. Three patients had a retreatment with balloon angioplasty.

\section{CONCLUSION}

Preliminary results of INTRASTENT proved complication and restenosis rates comparable with other published data of single centre case series. Statistical analysis to evaluate correlations between adverse events, clinical and technical factors is ongoing.

\section{MA07:11:4}

\section{INTRACRANIAL ARTERY STENOSIS TREATED BY ANGIOPLASTY WITH BALLON AND/OR STENT IN 37 PATIENTS}

G. Andrade ${ }^{1}$, $\underline{\mathrm{S} . \text { Bracard }}^{1}$, R. Anxionnat ${ }^{1}$, P. Lebedinsky ${ }^{1}$, N. Pinheiro $\mathrm{Jr}^{1}$, C. Macedo De Freitas ${ }^{1}$, L. Picard ${ }^{1}$ Department of Diagnostic and Interventional Neuroradiology. University Hospital, Nancy, France

\section{PURPOSE}

The symptomatic intracranial stenosis are rare and poor prognostic lesions. We presented thirty-seven patients who had symptomatic intracranial stenosis, whom twenty-nine were treated with angioplasty and nine with angioplasty and stent.

\section{MATERIALS AND METHODS}

In this study, between 1996 and 2006, we included thirty-seven patients with forty intracranial stenosis at the internal carotid artery (11), middle cerebral artery (8), basilar artery (16) and vertebral artery (5). These atherosclerotic stenosis were all superior to $70 \%$ (mean $83.5 \%$ ) at angiography and symptomatic even after the antiplatelet treatment. The angioplasty technique was used with an undersized angioplasty ballon and with a slow and progressive insuflation.

RESULTS

The treatment was always possible with a significant reduction of the stenosis level inferior than $50 \%$ in two cases. The level of middle stenosis was $36.8 \%$ after the treatment. There were complications in three cases: two reperfusion haematomas and one dissection with thrombosis. The minimum follow up was six months and we found just one case of restenosis superior than $50 \%$ after the angioplasty without stent. During the follow up, none of the patients presented an ischemic vascular event.

\section{CONCLUSION}

The treatment of intracranial stenosis is efficient with a low risk of complications. The technique is still on discussion. In our series, the percentage of restenosis after the angioplasty is low.

\section{MA07:11:5}

\section{INTRACRANIAL STENTING IN THE TREATMENT OF WIDE-NECKED ANEURYSMS}

M. Leonardi ${ }^{1}$, M. Dall'olio ${ }^{1}$, P. Cenni ${ }^{1}$, L. Raffi ${ }^{1}$, L. Simonetti ${ }^{2}$

${ }^{1}$ Neuroradiology, Bellaria Hospital, Bologna, Italy;

${ }^{2}$ Neuroradiology, Maggiore Hospital, Bologna, Italy

\section{SUMMARY}

We positioned the following self-expanding stents certified for intracranial application: 16 Neuroform (Boston Scientific), three INX (Medtronic), one Leo (Balt). 6F calibre femoral introducers and guiding catheters were used for stent placement changing to $5 \mathrm{~F}$ calibre introducers and guiding catheters (Envoy, Cordis) for the Neuroform 2 and 3 stents. All procedures were carried out under general anaesthesia and heparinization. Our pharmacological protocol consisted of adjunctive treatment with anti-aggregants during the interventional procedure and for the following six months, without pre-medication. From November 2000 to August 2006 we treated 28 patients $(27 \mathrm{~F} / 1 \mathrm{M})$ with giant wide-necked aneurysms and one dissecting basilar artery aneurysm requiring the placement of 29 stents.

We successfully positioned 20 stents: 11 stents combined with coils (8 immediate; 3 late) with complete exclusion of the aneurysm from the circulation in seven cases and subtotal exclusion in four; nine stents not followed by embolization with complete exclusion of the aneurysm from the circulation in six cases and subtotal exclusion in three. Stenting was not possible in nine cases due to extreme vessel tortuosity and the poor flexibility of release systems for the first stents. No late stent occlusion or subarachnoid haemorrhage were encountered after treatment.

\section{MA07:11:6}

\section{ENDOVASCULAR TREATMENT OF MCA EMBOLISM BY SELF EXPANDABLE STENTS}

T. Mayer, S. Müller-Schunk, M. Wiesmann, H. Brückmann Klinikum Grosshadern, Munich, GERMANY 


\section{PURPOSE}

If intravenous fibrinolysis is contraindicated or has failed, endovascular recanalisation of MCA embolism is used. Especially after i.v. fibrinolysis mechanical recanalisation by aspiration and retriever devices is an option. But even by these means, recanalisation sometimes will not be achieved due to clot adherence, extension, or composition. Whereas balloon dilatation of a thrombus may cause fragmentation and distal embolism, the use of a stent can fix the thrombus and partially recanalize the artery. A selfexpandable stent need not to be completely expanded to the original vessel size as it is the case for balloon expandable stents, which could fragment the thrombus.

METHODS

We retrospectively analyzed prospectively collected material of endovascular treated embolic MCA occlusions. All patients treated by selfexpanding intracranial stents after failure of the Penumbra aspiration and retraction system or failure of the use of a Goose Neck snare and coaxial catheter aspiration were included. I.v. tirofiban was used for platelet inhibition during and after stenting.

\section{RESULTS}

In seven patients a Wingspan stent (6 patients) or Leo stent (1 patient) was implanted in the arterial segment, which was occluded by remaining thrombus. Occlusion sites were Carotid-T (2), M1 (3), M2 (1), M3 (1).

In all cases a TIMI 2 or 3 recanalisation was achived. In 3 patients an overstented branch remained occluded. No distal embolisation occurred. One patient suffered a fatal intracranial hematoma. Four patients achieved MRS 0-2.

CONCLUSIONS

Selfexpandable stents and i.v. infusion of tirofiban can be used to fix an embolus to the vessel wall and recanalize the carotid-T, the MCA, or its branches. Larger studies seem to be justified.

\section{MA07:11:7}

\section{ENDOVASCULAR RECANALIZATION} OF INTRACRANIAL ARTERIES USING MECHANICAL RECANALIZATION TECHNIQUES: TECHNICAL NOTES AND PRELIMINARY RESULTS

\section{Wiesmann, S. Müller-Schunk, H. Brückmann, T. Mayer Universitätsklinikum München, Munich, Germany}

\section{INTRODUCTION}

Fibrinolysis is an established treatment in acute embolism or thrombosis of intracranial arteries. If contraindicated or unsuccessful, mechanical recanalization is used. Presently, there are devices available for clot aspiration as well as mechanical clot retrieval. We report our in-house results from a cohort of patients treated using mechanical recanalization devices.

METHODS

Included in this analysis were 11 patients who have been treated at our institution over the last 4 months using mechanical recanalization devices. Endovascular procedures were performed using either clot aspiration, or clot aspiration combined with devices for mechanical clot retrieval. Patients suffered from occlusion of the middle cerebral artery $(n=7)$, the basilar artery $(n=2)$, or both internal carotid artery and middle cerebral artery $(n=2)$. In these 11 cases a total of 30 recanalization procedures were performed including clot aspiration $(n=15)$, mechanical clot retrieval $(n=11)$, and stenting $(\mathrm{n}=4)$.

\section{RESULTS}

Mechanical recanalization was successful in 8 of 11 cases. In 4 of these 8 cases a good result was achieved only by a combination of different recanalization techniques. There were no iatrogenic complications (e.g., perforation, dissection, obstruction). Different techniques to improve the success rate of the techniques used will be discussed.

\section{CONCLUSIONS}

The combined use of different techniques for mechanical endovascular recanalization represents an important therapeutic alternative in patients suffering from acute stroke.

\section{MA07:11:8}

TREATMENT OF INTRACRANIAL STENOSIS USING THE WINGSPAN-STENT \{A SINGLE CENTER EXPERIENCE IN 55 PATIENTS

\author{
R. Klucznik, D. Meyer, F. Nasseri, \\ G. Benndorf \\ The Methodist Hospital, Weill Cornell University, Houston, \\ New York, USA
}

\section{PURPOSE}

The purpose of this study is to report on a single center experience in using a self-expanding stent WWingspan Stent system, Boston Scientific/Target\} for endovascular treatment of intracranial stenoses.

\section{MATERIAL AND METHODS}

Fifty five patients $\{28$ males, 27 females, Age: $25-85$ years, mean age: 63.5 years $\}$ underwent treatment of 59 stenotic lesions of intracranial arteries $\{\mathrm{M} 1: 22, \mathrm{M} 2: 3$, ICA: 15, VA: 10, BA: 8, A1: 1 ) using a total of 66 stents (Wingspan, Boston Scientific/Target). After pre-dilatation of the stenosis using a PTA balloon catheter \{Gateway, Boston Scientific/Target), a single stent was deployed in 45 patients while 2 stents were placed in 9 patients with more than one stenosis and 3 stents placed in one patient. Stenosis prior to stent placement ranged from $45 \%-95 \%$ mean $76 \%$ \}.

\section{RESULTS}

Technical success was achieved in 58/59 lesions $\{98 \%\}$ in all locations with good anatomical results. Increase of vessel diameter was accomplished in 58/59 lesions with a remaining residual stenosis ranging from $0-63 \%$. Two patients developed major neurological complications: one died due to a progressive stroke, one was referred for rehabilitation. One patient developed a minor ipsilateral bleeding causing headache only. $17 / 55$ patients $\{31 \%\}$ had 6-months angiographic follow-up, $5 / 17$ restenoses $\{30 \%\}$ showed restenoses between $25 \%$ and $78 \%$. Among them, one patient developed a new stroke while four patients remained asymptomatic. There were two asymptomatic complete occlusions $\{11.8 \%\}$. 


\section{CONCLUSIONS}

The use of the self-expanding Wingspan stent for treatment of intracranial stenosis is technically feasible and safe. The short term angiographic follow-ups show a relatively high restenosis and occlusion rate. More clinical and angiographic follow-up data are required for critical assessment of this new treatment modality.

\section{MA07:11:9}

\section{SELF-EXPANDABLE WINGSPAN STENT FOR INTRACRANIAL STENOSES TREATMENT: TWELVE MONTHS EXPERIENCE.}

M. Isalberti, N. Nuzzi, C. Cinnante, C. Sina, A. Costa,

S. Avignone, P. Machì, V. Branca

Neuroradiologia Diagnostica ed Interventistica Fondazione Ospedale Maggiore Policlinico Mangiagalli Regina Elena - IRCCS Milano, Italy

\section{INTRODUCTION}

The endovascular treatment of intracranal arterial stenoses in the past was limited by using cardiac stents; slightly more than a year a self-expandable stent (Wingspan - Boston Scientific), with open cells, is available. It has CE label for intracranial use. Aim of the study is to show 10 months of our experience.

MATHERIAL AND METHODS

From March 2006 to March 2007 we treated 12 intracranial stenoses in 10 patients using Wingspan stent: 5 patients with anterior circulation stenoses (totally 6 stenoses, 5 intracranial extradural and 1 extracranial) and 5 with posterior circulation stenosis (6 intradural stenoses). In all cases, except for two of them, balloon angioplasty was performed before deployment; in a single case dilatation after deployment was performed. Angiographic follow-up at seven days, three, six and twelve months was scheduled.

\section{RESULTS}

In all cases stents were deployed. In one case, due to wrong deployment of the first stent, a second stent release was needed. In a patient with two stenoses and proximal pseudo-aneurysms, two Wingspan stents overlapped with a Neuroform stent were deployed in order to cover both stenoses and pseudo-aneurysm. In a single case the preliminary angioplasty provoked dissection, well repaired by the stent. In the remaining patients no immediate angiographic complications were demonstrated.

One case had worsening of neurological symptoms after wake-up, with improvement during hospital stay.

In a single case the stent deployment provoked a pseudo-aneurysm of the carotid siphon, and carotid occlusion, well tolerated, was necessary.

In two of 6 patients, with angiographic follow-up, slight narrowing was observed, but no other treatments were required; 4 patients are waiting follow-up.

\section{CONCLUSIONS}

Wingspan stent is designed for tortuous intracranial vessels; complication and recurrence rate seems low, but further studies are mandatory.

\section{MA07:11:10}

\section{TESTING PROCEDURE FOR QUANTIFICATION OF LUMENVISIBILITY OF IN-STENT RESTENOSIS USING MAGNET RESONANCE IMAGING (MRI)}

G. Schaefers ${ }^{1}$, G. Schoenwald ${ }^{2}$, A. Melzer ${ }^{3}$

1 MR:comp GmbH, MR Safety Testing Laboratory, Gelsenkirchen, Germany; ${ }^{2}$ University of Applied Sciences FH Gelsenkirchen, Gelsenkirchen, Germany; ${ }^{3}$ Institute for Medical Science \& Technology Universities Dundee \& St. Andrews \& Ninewells Hospital, Dundee, United Kingdom

\section{PURPOSE}

Determination of in-stent restenosis is of diagnostic interest. A test procedure for quantification of artificial in-stent restenosis and comparison of stents was evaluated to determine the lumenvisibility using MRI.

METHODS

3 carotid stents $(30-34 \times 8 \mathrm{~mm})$ were used: Strecker Tantalum (open cell), Boston Scientific; Nitinol Precise RX \& 316L Palmaz Genesis, (closed cell), Cordis). A T1 relaxating material for simulating the artificial restenosis was pre-investigated of $\mathrm{T} 1$ compared to a pig's aorta at $1 \& 1.5$ Tesla. Stents were placed into silicone tubes with artificial stenosis of $50 \%, 75 \%$ and $90 \%$. The stented tubes were positioned in a static phantom filled with $0.9 \% \mathrm{NaCl}$ solution doped with $0.2 \mathrm{mmol} / \mathrm{kg}$ contrast medium (Gadovist, Schering). The stents were placed parallel to B0 into the head coil of a 1 Tesla MR system (Intera, Philips Medical Systems) We ran gradient echo (GRE), T1 \& T2-weighted spin echo (SE) and 3D balanced Fast Field Echo (3DbFFE) pulse sequences. Signal intensities in- and outside the stents were measured using DICOM tools. Artifacts were rated qualitatively.

\section{RESULTS}

The in-stent lumen of the $316 \mathrm{~L}$ stainless steel closed cell stent was completely covered by artifacts. The nitinol stent shielded the signal high-grade. In spite of signal reduction the artificial restenosis were visible in the T2-SE. Using the 3DbFFE, the Tantalum stent was depicted with actual value at $50 \%$ and $75 \%$ degrees of stenosis. Merely with $90 \%$ artificial restenosis there was a divergence of $2.5 \%$ to $87.5 \%$.

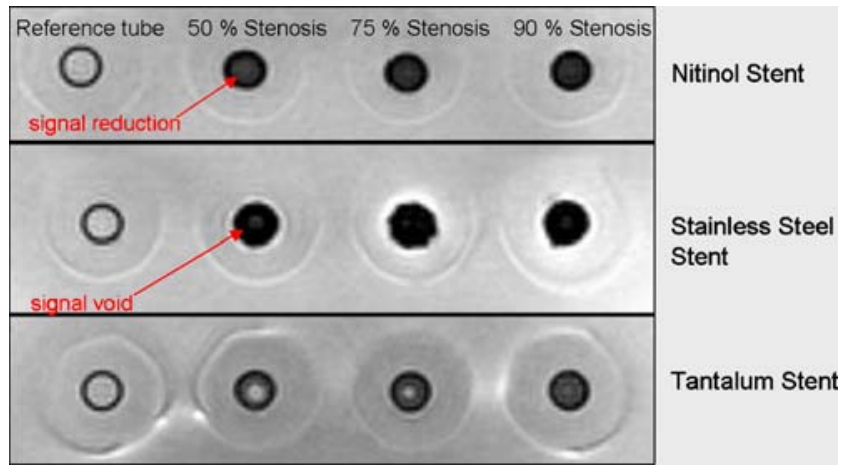




\section{CONCLUSIONS}

This test procedure allows a quantitative determination of in-stent restenosis by analysis of the lumenvisibility with MRI. It is obvious that the stent material and design has great influences on the visibility of an in-stent restenosis. With the choice of a low-artifact stent material and a design with low radio frequency shielding, a quantification of instent restenosis is possible.

\section{MA08}

16.45-17.40 - Head and neck - contrast agents

\section{MA08:12:1}

\section{HEAD AND NECK TRAUMA MANAGEMENT: TRANSFERRING NEURORADIOLOGICAL EXPERTISE INTO DAILY PRACTICE}

\section{J. Zajaczek, G. Goetz, F. Donnerstag, H. Matthies,} M. Behrends, T. Kupka, B. Haubitz, H. Lanfermann Hannover Medical School, Hannover, Germany

Trauma is a critical event where efficient management is necessary. If the head and neck region is involved it is frequently of vital importance to give correct diagnoses rapidly and bring adequate therapy on the way. Besides intracranial injuries, traumata of the midface are a diagnostic and therapeutic challenge not only for the unexperienced. Due to complex anatomy and multiplicity of bony fissures, pathology is not easy to be detected.

A web-based case-book, called "Schoolbook-Nightingale", is generated in order to aid physicians especially in the acute phase of trauma and during night duty. It provides reference cases for comparison in the acute phase as well as education in specialised neuroradiological knowledge.

For the generation of "Schoolbook-Nightingale" the "Schoolbook", a content management system, is reutilised. The content is stored in a database, layout is defined by a PHP application. Webpages are generated dynamically from a PHP application. Schoolbook is realized on a Linux system with the Apache web server, the programming language PHP, and the database MySQL.

History and diagnostic data of patients suffering from trauma of the head and neck region are integrated multimedially, consisting of texts, images, and video.

A multimedial reference case-book is generated, mainly dealing with information about treatment of patients with trauma of the head and neck region with special focus on midfacial trauma. Several case-histories cover the full range of traumata from minor to severe. Thanks to the didactic structure "Schoolbook-Nightingale" can be used for reference purposes as well as for structured training.

The newly created case-book provides fundamental and comprehensive information about management and diagnostic investigation of trauma of the head and neck region. It is useful both for reference and optimization of diagnostic strategy and improvement of diagnostic findings.
The content is available on the worldwideweb, thereby providing the transfer of neuroradiological expertise into daily practice areawide and full-time.

\section{MA08:12:2}

\section{PROSPECTIVE STUDY TO DEVELOP BEST PREDICTIVE MODEL FOR HEAD CT IN NON-TRAUMA CASES}

N. Young ${ }^{1}$, I. Tan ${ }^{1}$, D. Sindhusake ${ }^{2}$, R. Dowsett ${ }^{3}$

${ }^{1}$ Department Of Radiology, Westmead Hospital, Sydney, Australia;

2 Department Of Public Health, Westmead Hospital, Sydney, Australia; ${ }^{3}$ Department Of Accident \& Emergency, Westmead Hospital, Sydney, Australia

\section{PURPOSE}

To develop a clinical model that best predicts significant cranial CT findings in patients acutely presenting with non-trauma symptoms.

METHODOLOGY

Prospective study from 2002 to 2006, at a single adult university hospital, of patients presenting in accident and emergency. Presenting clinical symptoms and signs were correlated with CT findings. Statistical analysis of potential predictors in a logistic regression, with odds ratio at $95 \%$ confidence interval, with multivariable modelling.

RESULTS

1,911 patients studied, in age range 16-92 years. Clinically significant CT findings included acute infarct, haemorrhage, malignancy, hydrocephalus, infection. The presence of GCS $<14$, weakness, sensory loss, history of malignancy, headache, aphasia and diabetes were statistically significant. Modelling these factors permits a sensitivity of $99.3 \%$, false negative of $0.7 \%$ when using these presenting criteria for eliciting significant $\mathrm{CT}$ findings. Other presenting factors were not significant, including age, vomiting, confusion, and ataxia.

\section{CONCLUSION}

Statistically based clinical modelling can be a predictor for need for immediate, vs delayed, head CTs in a non-trauma setting.

\section{MA08:12:3}

\section{COMPUTED TOMOGRAPHY GUIDED PERCUTANEOUS CORE BIOPSY OF DEEP FACE AND SKULL BASE LESIONS}

N. Chaudhary, I. Devagnanam, S. Connor

King's College Hospital, London, United Kingdom

\section{PURPOSE}

The utility of percutaneous core biopsy for the evaluation of deep face and skull base lesions is not well documented. The availability of histological sampling (rather than fine needle aspiration cytology) increases diagnostic accuracy for benign disease and eliminates requirement for periprocedural cytological 
expertise. We present our experience of computed tomography (CT) guided core biopsy of deep face and skull base lesions in fourteen patients.

\section{MATERIALS AND METHODS}

Fourteen patients who underwent a CT guided percutaneous core biopsies of deep face and skull base lesions at a tertiary neurosciences centre from 2003 to 2006 were retrospectively analysed. The lesions were sited within the skull base (4), parapharyngeal and retropharyngeal spaces (3), infratemporal (2), pterygopalatine fossae (2), and deep lobe of parotid (3). The procedure was performed under general anaesthetic or intravenous sedation. A 22/18 g CookTM coaxial core biopsy system was used. Sigmoid notch (subzygomatic), retromaxillary, suprazygomatic, and transparotid needle approaches were used. The diagnostic accuracy was assessed by a positive histology result which corresponds to the operative specimen or treatment response.

\section{RESULTS}

Females:Males=5:9 (age 5-83). Median follow up: 12 months (range 4-24). Number of passes: median 3 (range 2-5).

No procedural complications were encountered. A histological diagnosis was obtained in all the patients from the obtained specimens. The pathological diagnosis was confirmed in 3 by surgical excision and in 6 by predictable treatment response $(9 / 10$ patients: 4 deaths), giving a diagnostic accuracy of $90 \%$.

\section{CONCLUSION}

CT guided percutaneous deep face core biopsy is a robust technique with good diagnostic accuracy.

\section{MA08:12:4}

\section{STS SCLEROTHERAPY FOR PATIENTS WITH VENOUS MALFORMATION: CLINICAL RESULTS WITH PATIENT-BASED AND MRI-BASED EVALUATION}

N. Tanaka ${ }^{1}$, T. Abe ${ }^{1}$, Y. Uchiyama ${ }^{1}$, S. Nagata ${ }^{1}$, $\overline{\text { N. Hayabuchi }}{ }^{1}$, K. Kiyokawa ${ }^{1}$

Kurume University Hospital, Kurume, Japan

\section{PURPOSE}

Purpose of this study is to evaluate the safety and efficacy of percutaneous sclerotherapy using sodium tetradecyl sulfate (STS) for patients with venous malformation (VM) and to clarify the appropriate patient groups for the therapy.

\section{MATEARIALS AND METHODS}

We treated thirty-four consecutive patients with VMs from Feb. 2001 to Oct. 2006. Locations of the VMs were cheek in 14, lip in 5 , face in 5, upper ext. in 4, lower ext. in 3, and 3 in others. Mean age of the patients was 20.3 years old (6 months to 66 y.o.), Mean number of the sessions were 3.08 ( 1 to 11 sessions). Fluoroscopic guidance in the DSA unit was used under general anesthesia in infants and local anesthesia in adults. The lesions were directly punctured with 23-gauge needle and a mixture of 3\% STS and contrast media was injected fluoroscopically without extravasation.
We evaluated indication for the therapy (pain, cosmetic problem, functional problem), the previous treatments (treated or not), and MRI classifications before therapy. The therapeutic responses were evaluated by both patients-evaluation and MRI-evaluation into excellent, good, and poor.

\section{RESULTS}

Results by patient-based evaluation were significantly better than those by MRI-based evaluation $(p<0.01)$. Sclerotherapy were judged significantly more beneficial in patients with indication of pain or functional problems than in patients with cosmetic reasons $(\mathrm{p}<0.01)$.

Conclusion: Sclerotherapy by STS for was judged beneficial in $62 \%$ of the patients by MRI evaluation and in $79 \%$ of the patients by patient-evaluation. Pain is a good indication for sclerotherapy by STS.

\section{MA08:12:5}

\section{MR STUDY OF VENTRO-LATERAL MEDULLARY COMPRESSION AND HYPERTENSION IN HEMIFACIAL SPASM AND HEALTHY CONTROLS}

L. Chan ${ }^{1}$, E. Lee ${ }^{1}$, E. Tan ${ }^{2,3}$

${ }^{1}$ Singapore General Hospital, Singapore, Singapore;

${ }^{2}$ National Neuroscience Institute, Singapore, Singapore;

${ }^{3}$ SingHealth Pte Ltd, Singapore, Singapore

\section{PURPOSE}

In a case control study, we evaluate for ventro-lateral medullary (VLM) compression in hypertensive hemifacial spasm (HFS) patients and in healthy controls using advanced MRI/A imaging techniques.

\section{METHOD AND MATERIALS}

Hypertensive HFS patients and a group of age, gender and race matched normotensive healthy individuals were recruited. The presence of VLM compression was analyzed using constructive interference at steady state (CISS) MR and 3 dimensional time-offlight MRA techniques. The degree of VLM compression was quantified using a severity scale with grading from 0 to 3 . Chisquare and student $\mathrm{t}$ test was used to compare the various categorical and numerical variables.

\section{RESULTS}

A total of 50 subjects underwent imaging. Their mean age was about 53 years old and about $60 \%$ of them were women. Majority of HFS patients had left sided symptoms. Analysis of the group of 25 HFS and 25 controls revealed that the prevalence of VLM compression was higher in hypertensive HFS compared to controls. In addition, there was a trend towards a greater severity of VLM compression in the HFS group, though there was no great difference between left and right sided compression.

\section{CONCLUSION}

VLM compression was associated with hypertension in HFS and a greater severity of VLM compression was observed in patients compared to controls. 


\section{CLINICAL RELEVANCE/APPLICATION}

Our findings have clinical implications in the management of some at-risk patients.

\section{MA08:12:6}

\section{MDCT IN OBSTRUCTIVE SLEEP APNOEA SYNDROME EVALUATION OF THE UPPER AIRWAY AND CT CEPHALOMETRIC ANALYSIS: WORK IN PROGRESS}

N. Kwatra, J. Bapuraj, A. Gupta, A. Aggarwal, N. Khandelwal, P. Singh

1 Postgraduate Institute of Medical Education and Research, Chandigarh, India; ${ }^{2}$ University Hospital. University Of Michigan, Ann Arbor, USA; ${ }^{3}$ Postgraduate Institute Of Medical Education And Research, Chandigarh, India; ${ }^{4}$ Postgraduate Institute Of Medical Education And Research, Chandigarh, India; ${ }^{5}$ Postgraduate Institute Of Medical Education And Research, Chandigarh, India; ${ }^{6}$ Postgraduate Institute Of Medical Education And Research, Chandigarh, India

Obstructive Sleep Apnea Syndrome (OSAS) is a major public health problem affecting about $2-4 \%$ of the middle-aged population. The upper airway geometry in patients with OSAS differs from those in normal subjects which is amenable to cross sectional imaging

\section{OBJECTIVES}

- To prospectively assess the upper airway anatomy of OSAS patients by multi-detector row computed tomography (MDCT) and compare these findings with age and sex matched controls

- To assess the changes in the anatomical parameters in those OSAS patients (included in the initial study) who undergo surgery. MATERIALS AND METHODS

Subjects of either sex and more than 18 yrs of age with signs and symptoms suggestive of OSAS and a diagnostic sleep study with a Respiratory distress index (RDI) $>5$ are being enrolled in the study. We intend to include a total of 30 OSAS subjects.

All the diagnosed subjects will be undergoing a detailed CT evaluation. Controls for the study are being chosen from amongst the patients presenting to our department for Head/Neck CT for an unrelated pathology.

After obtaining the topogram and base images of the region of interest, these are exported to the work station for further analysis. Sagittal and coronal reconstruction and volume rendering are done. Anatomical landmarks are identified and various parameters evaluated. The cephalometric parameters include linear bony measurements, angular bony measurements and soft tissue measurements. Additionally, the cross sectional areas and AP and lateral dimensions and few other parameters at various levels of the airway are also measured.

RESULTS

A very preliminary analysis of the data reveals that the parameters which are likely to achieve significant differences between the two groups once the study is completed include the mandibular hyoid distance (mean $1.26 \mathrm{~cm}$ in control group, $1.60 \mathrm{~cm}$ in OSAS group), the tongue length (mean $6.89 \mathrm{~cm}$ in control group, $7.31 \mathrm{~cm}$ in OSAS group), soft palate length (mean $3.62 \mathrm{~cm}$ in control group, $4.10 \mathrm{~cm}$ in the OSAS group) retropalatal cross sectional area (mean $1.99 \mathrm{sq} \mathrm{cm}$ in controls, $1.24 \mathrm{sq} \mathrm{cm}$ in cases), retropalatal antero-posterior and lateral dimensions.

\section{CONCLUSION}

By means of this study, we intend to provide a comprehensive CT database which can add to our knowledge of the synchronous narrowings of the upper airway and demonstrate the "bottle-neck point". This will go a long way in furthering the understanding of the mechanisms of OSAS. Besides, the study intends to give volumetric structural substrate to the surgeons before they embark upon surgery for OSAS.

\section{MA08:12:7}

\section{IMAGING OF INTRALABYRINTHINE SCHWANNOMAS: A RETROSPECTIVE STUDY OF 51 CASES WITH EMPHASIS ON LESION GROWTH}

A. Tieleman $^{1}{ }^{\text {, J. Casselman }}{ }^{1,2}$, T. Somers ${ }^{2}$, $\overline{\text { R. Kuhweide }}^{1}$, B. De Foer ${ }^{2}$, E. Offeciers ${ }^{2}$

${ }^{1}$ AZ St-Jan Brugge AV, Bruges, Belgium; ${ }^{2}$ AZ St-Augustinus Antwerp, Antwerp, Belgium

\section{PURPOSE}

Intralabyrinthine schwannomas (ILS) are benign tumors arising from the perineural schwann sheath of the intralabyrinthine branches of the vestibulocochlear nerve and initially do not extend in the internal auditory canal (IAC) [1].

Little is still known about their prevalence, occurence and patterns of growth.

This study reviewed the largest series of ILS reported until now. METHODS

Exact lesion location and growth patterns of all ILS unequivocally seen on at least one dedicated MR examination between februari 1991 and april 2007 were evaluated. The number of the CPA/IAC schwannomas detected during the same period was calculated to get an idea of the prevalence of ILS.

RESULTS

51 patients were included. ILS were located on the right side (43\%), left side $(55 \%)$, or bilateral $(2 \%)$.

Exact lesion location is presented in table1. Of the 51 ILS 41 were found in the cochlea $(80 \%), 7$ were found in the vestibulum and/or semicircular canals $(14 \%)$ and 3 were found in both the cochlea and vestibular labyrinth $(6 \%) .63 \%$ of intracochlear schwannomas were confined to the scala tympani (ST), 37\% involved both ST and scala vestibuli (SV). All lesions enhanced strongly on the Gadolinium-enhanced T1-weighted images and caused a loss of the high signal intensity of the intralabyrinthine fluid on the heavily T2-weighted images. Folluw-up MR exams were available in 26 of 51 patients. Tumor growth was observed in $58 \%$ of these (table 2).

For every 8.6 IAC/CPA schwannomas one ILS was found, compatible with an estimated prevalence of $11 \%$. 
Twelve lesions were surgically removed. The reasons for resection were tumor growth in 3 cases $(25 \%)$, tinnitus in 2 cases $(16.7 \%)$, vertigo in 1 case (6.3), inability of 1 patient to live with the idea of a "benign" tumor $(8.3 \%)$ and 'preventive' in 5 patients $(8.3 \%)$. DISCUSSION AND CONCLUSION

Diagnosis was confirmed in $61 \%$ by means of pathological examination and/or serial MR studies.

Predilection place for development is the anterior cochlea, the transition area between basal and second turn. They develop from the ST and grow into the SV. Involvement of the vestibular labyrinth is far less frequent. Intracochlear schwannoma's first grow within the cochlear turns and later extend into vestibule and/or IAC. Vice versa, vestibular schwannoma first occupy the vestibular labyrinth and then progress into the cochlea's basal turn.

Both prevalence and tumour growth seem higher than previously reported [2].

\section{REFERENCES}

1. Neff BA, et al. (2003) Otol Neurotol 24: 299-307

2. Kennedy RJ et al. (2004) Otol Neurotol 25: 160-167

Table 1. Intralabyrintine distribution of the 51 ILS at initial MRI.

\begin{tabular}{|c|c|}
\hline intial lesion location & $\begin{array}{l}\text { no. of patients } \\
(\%)\end{array}$ \\
\hline Intracochioat & $41(80.4)$ \\
\hline basalten & $4(7.8)$ \\
\hline ST & $3(5.9)$ \\
\hline $\mathrm{SV}+\mathrm{ST}$ & $1(2)$ \\
\hline socond tum & $10(19.6)$ \\
\hline ST & $0(17.7)$ \\
\hline $\mathrm{SV}+\mathrm{ST}$ & $1(2)$ \\
\hline apikal tum & $6(11.8)$ \\
\hline ST & $4(7.8)$ \\
\hline $\mathrm{SV}+\mathrm{ST}$ & $2(3.9)$ \\
\hline basal tum + socond Lum & $16(31.4)$ \\
\hline ST & $8(16.5)$ \\
\hline ST and SV of both tums & $4(7.8)$ \\
\hline ST basal tum and $\mathrm{ST}+\mathrm{SV}$ socond tum & $3(5.9)$ \\
\hline $\mathrm{ST}+\mathrm{SV}$ basalitum and $\mathrm{ST}$ socond tum & $1(2)$ \\
\hline apikal tum+tocond $\mathrm{tmm}$. & $5(1.5)$ \\
\hline ST & $2(3.0)$ \\
\hline sv & $1(2)$ \\
\hline ST apikal tum and SV+ST socond fum & $2(3.9)$ \\
\hline Intravestoular & $7(13.7)$ \\
\hline anterior portion & $1(2)$ \\
\hline semicroular canals (SCCs) & $2(3.9)$ \\
\hline (anleriortposterior) vestibuo + SSC & $3(5.9)$ \\
\hline antorior vostoub * SCCS & $1(2)$ \\
\hline Anterior vestbulo + all cochloar tums & $3(5.9)$ \\
\hline
\end{tabular}

Table 2. Patterns of lesion growth $(\mathrm{n}=15)$.

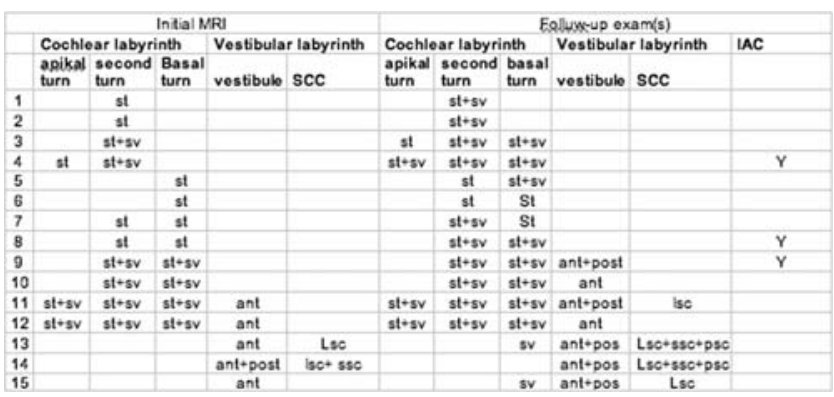

\section{MA08:12:8}

\section{GADOLINIUM CONTAINING CONTRAST AGENTS THEIR POTENTIAL ROLE IN THE PATHOGENESIS OF NSF}

M. Sieber, J. Walter, T. Frenzel, H. Weinmann, H. Pietsch Bayer Schering Pharma, Berlin, Germany

\section{PURPOSE}

Nephrogenic systemic fibrosis (NSF) is an acquired, idiopathic disorder observed only in patients with severe renal dysfunction. The cuteneous findings in NSF patients are characterized by skin thickening, infiltration of CD34+ cells and increase in collagen bundles. Recently, several publications suggested a relationship between the administration of Gd -based CA (predominantly Gadodiamide (Omniscan $\left.{ }^{\circledR}\right)$ ) and the occurrence of NSF.

The objective of this preclinical study was to investigate the potential of various Gadolinium-chelates to release Gadolinium in vivo and to evaluate their potential to induce NSF.

METHODS AND MATERIALS

Clinically used extra cellular Gd-containing contrast agents (Gd-DTPA (Magnevist $^{\circledR}$ ), Gadobutrol (Gadovist $\left.{ }^{\circledR}\right)$, Gadodiamide (Omniscan ${ }^{\circledR}$ ), Gadoversetamide $\left(\right.$ Optimark $\left.^{\circledR}\right)$, Gd-BOPTA (Multihance $\left.{ }^{\circledR}\right)$, GdDOTA $\left(\right.$ Dotarem $\left.^{\mathbb{R}}\right)$ ) were tested in a subchronic toxicity study in rats. The animals received i.v. injections at a dose of $2.5 \mathrm{mmol} \mathrm{Gd} / \mathrm{kg}$ five times per week for four weeks. Animals were examined daily regarding pathologic findings and were sacrificed 5 days after the last injection. Histopathology of the skin was evaluated and the Gd-concentration in various tissues measured. In addition electron microscopy combined with energy dispersive spectroscopy was performed.

RESULTS

In rats a high exposure to the contrast agents was achieved by daily injections, similar to the situation in patients with severe renal dysfunction,. Macroscopic and microscopic findings (fibrosis and infiltration of CD34+ cells) in the skin were observed only in the animals treated with Gadodiamide (Omniscan ${ }^{\circledR}$ ) and were associated with very high $\mathrm{Gd}$ concentration in the skin. In this lesions Gd-deposits were detected in electron microscopy.

\section{CONCLUSION}

The used animal model was an appropriate tool to investigate differences between various Gd-chelates regarding their risk to accumulate in tissue and trigger NSF-like skin lesions. NSF like skin lesions were only observed after

\section{MA08:12:9}

\section{IDENTIFICATION OF A POTENTIAL TRIGGER FOR THE INDUCTION OF NEPHROGENIC SYSTEMIC FIBROSIS?}

H. Pietsch, T. Frenzel, J. Walter, H. Weinmann, M. Sieber Bayer Schering Pharma, Berlin, Germany

\section{PURPOSE}

Nephrogenic Systemic Fibrosis (NSF) is an acquired, idiopathic disorder in patients with severe renal dysfunction, characterized by skin thickening primarily on the extremities and the trunk.

Recently, several publications suggested a possible relationship between the administration of Gadolinium (Gd)-based contrast agents and NSF, in particular Gadodiamide (Omniscan $\left.{ }^{\circledR}\right)$. The objective of this study was to investigate two potential mechanisms currently under discussion as possible triggers for NSF: relase of $\mathrm{Gd} 3$ + ions from the Gd-chelate complex and accumulation in tissue or depletion of endogenous metal ions (e.g., Zinc) by the ligand. 


\section{METHODS AND MATERIALS}

Gadodiamide and Gadoversamide, the drug substances of Omniscan ${ }^{\circledR}$ and OptiMARK ${ }^{\circledR}$ respectively, were used as formulations with $0 \%$, $5 \%$ and $10 \%$ (excess) content of Gd-free ligand to modulate different risks of $\mathrm{Gd} 3+$-ion release and depletion of endogenous ions. HanWister rats were injected daily i.v at a dose of $2,5 \mathrm{mmol} \mathrm{Gd} / \mathrm{kg}$ b.w. for up to 20 days. Histopathology, immuno-histochemistry, electron microscopy combined with energy-dispersive spectroscopy of the skin was performed and concentrations of $\mathrm{Gd}, \mathrm{Zn}$ and $\mathrm{Cu}$ in skin, liver, femur and serum were measured.

RESULTS

The most severe skin lesions and the highest Gd concentrations in skin observed after injection of compounds with the highest risk for release of $\mathrm{Gd} 3+$ ions ( $0 \%$ excess ligand). No skin lesions and significantly lower Gd concentrations were observed after injection of compounds with the highest risk for generating a depletion of endogenous metal ions (compounds with $10 \%$ free ligand). No decrease of Zinc and $\mathrm{Cu}$ values was observed in any of the examined tissues (including serum).

\section{CONCLUSION}

No loss of endogenous ions was observed, indicating that depletion of endogenous metal ions does not play a causative role in the pathogenesis of NSF. Furthermore the results strongly suggest that release of $\mathrm{Gd} 3+$ and accumulation in the skin tissue trigger the onset of NSF.

\section{Scirocco - Libeccio Room}

\section{SL05}

09.00-10.00 - Spinal \& spinal cord

\section{SL05:13:1}

\section{MR IMAGING OF THE SPINE: A COMPARISON AT $1.5 \mathrm{~T}$ AND $3 \mathrm{~T}$}

\section{Garcia Alzamora, I. Mader}

Section of Neuroradiology, University Hospital Freiburg, Freiburg, Germany

\section{PURPOSE}

In spite of the many advantages of high field MRI, imaging at $3 \mathrm{~T}$ is negatively influenced by many issues including increased specific absorption rate (SAR), prolonged $\mathrm{T} 1$ at reduced $\mathrm{T} 2 *$ relaxation times and increased magnetisation transfer effects. The purpose of this study was to assess spine pathologies at a 1.5 and a 3 T system.

\section{METHODS}

19 patients were investigated on a $1.5 \mathrm{~T}$ and other 19 patients on a $3 \mathrm{~T}$ whole body MR system (SIEMENS, Vision and Tim Trio respectively). Three of the 35 patients were examined in both MR-Systems. Pathologies comprised 3 cervical and 3 lumbar disc herniations, 3 cavernomas, 3 syringes, 3 encephalitis disseminatae, 3 epidural metastases and 1 intradural tumor for each MR system, respectively. Routinely performed T2-TSE-weighted and sagittal non-contrast enhanced T1-TSE-weighted images were chosen for qualitative and quantitative analysis. The pixel size at $3 \mathrm{~T}$ was half or less than half of that at $1.5 \mathrm{~T}$ at equal acquisition times.

\section{RESULTS}

All investigated criteria, such as pulsation and flow artefacts, anatomic structures, overall image quality in $\mathrm{T} 1$ and $\mathrm{T} 2$, lesion conspicuity and SNR (signal-to-noise ratio) of the myelon in T1 and $\mathrm{T} 2$ were similar at both field strengths.

\section{CONCLUSIONS}

The theoretically nearly two-fold signal at $3 \mathrm{~T}$ was used to clinically image at a higher spatial resolution. The equality in subjective ranking and in quantitative analysis implies that imaging at $3 \mathrm{~T}$ can be fully implemented into clinical imaging without loss of investigation time.

\section{SL05:13:2}

\section{THE ROLE OF MRI IN THE EARLY DIAGNOSIS AND FOLLOW-UP OF PATIENTS WITH SPONDYLODISCITIS}

E. Kapsalaki, A. Balatsouka, A. Vassiou, I. Gatselis, G. Dalekos, I. Fezoulidis

University Hospital of Larissa, Larissa, Greece

\section{PURPOSE}

Spondylodiscitis is considered a relatively rare infection of the vertebral column. It represents $2-7 \%$ of all causes of osteomyelitis. Spondylodiscitis has been known since the ancient years and Hippocrates reported spinal osteomyelitis in $400 \mathrm{BC}$. The most common pathogen is $\mathrm{S}$. aureus. A serious complication of spondylodiscitis is the formation of epidural and paraspinal abscesses. Its early recognition and treatment is of paramount importance for the good outcome of these patients.

MATERIAL AND METHODS

In our current communication we present 12 patients $(7$ males, 5 females) who presented to our clinic with spondylodiscitis, over a 1 year period. Their mean age was 63.2 years. None of these patients had undergone any surgical procedures. Eight of our patients had diabetes mellitus. All patients underwent CT and MRI studies to identify the location and the extent of their disease.

\section{RESULTS}

The underlying pathogens identified in their blood cultures were Staph. aureus in 6 patients, Enterococcus fecalis in one, Pseudomonas aeruginosa in one, and Brucella in 3 patients. Spondylodiscitis involved the lumbar spine in 5 patients, thoracic spine in 3 , cervical spine in one and more than one locations in 3 patients. In addition, in 3 patients the infection was extended in the epidural and paraspinal space. The progress of the disease was evaluated with serum sed. Rate and CRP values and consecutive MRI studies. All patients were treated with prolonged course of antibiotics and external orthotic device. Clinical improvement preceded imaging improvement in 11/ 12 of our patients. Moreover, in 2 patients antibiotic regimen had to 
be changed due to disease progression demonstrated in MRI. One patient in our cohort needed surgical stabilization.

\section{CONCLUSION}

Advanced age and diabetes mellitus predispose the development of spondylodiscitis. MRI is the method of choice for early detection and evaluation of the therapeutic management of these patients.

\section{SL05:13:3}

\section{DIFFUSION WEIGHTED IMAGING IN SPONDYLODISCITIS}

\section{G. Callari ${ }^{1}$, A. Arrigo ${ }^{1}$, M. Furlan ${ }^{2}$}

1 Neuroradiology of Imperia - Ponente Ligure Neurosciences Department, Imperia, Italy; ${ }^{2}$ Neurology of Imperia - Ponente Ligure Neurosciences Department, Imperia, Italy

We present the case of a 81 years old woman with a primary brest carcinoma with extra-vertebral bone metastasis where an L3-L4 spondylodiscitis was suspected on the MRI. That diagnosis has been confirmed on a CT guided biopsia to be due to Staphylococcus Coagulase Negative and Klebsiella Pneumoniae.

The diagnostic MRI included the Diffusion Weighted Imaging (DWI) sequence designed for the Brian centred on the spine in the sagittal plane that showed a restricted diffusion area at the level of the disc infection but a very poor anatomical feature.

DWI sequence for the spine needs to improve from a technical point of view and our experience confirm that, as we know for the brain, this can be a very important tool in the differential diagnosis for infectious disease.

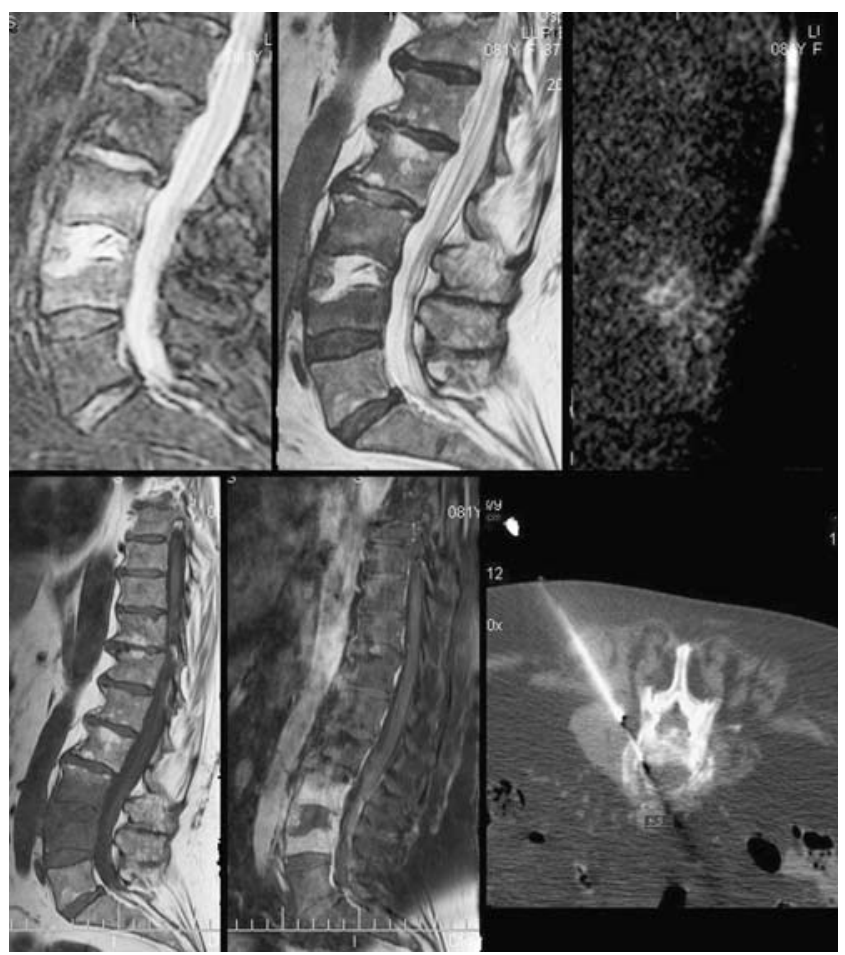

\section{SL05:13:4}

DIFFUSION TENSOR MR IMAGING (MRI) OF THE CERVICAL SPINAL CORD IN ASYMPTOMATIC HUMAN IMMUNODEFICIENCY VIRUS (HIV) - POSITIVE PATIENTS: PRELIMINARY RESULTS AT $3.0 \mathrm{~T}$

$\underline{\text { C. Mueller-Mang }}{ }^{1}$, T. Mang ${ }^{1}$, F. Cartes-Zumelzu ${ }^{1}$, M. Law ${ }^{2}$, M. Thurnher ${ }^{1}$

${ }^{1}$ Medical University Vienna, Department of Radiology, Vienna, AUSTRIA; ${ }^{2}$ Mount Sinai Medical Center, Department of Radiology, New York, USA

\section{PURPOSE}

Diagnosis of spinal cord involvement in patients with AIDS remains challenging using conventional MRI. The purpose of our study was:

1) To measure DTI metrics of the cervical spinal cord in asymptomatic HIV positive patients

2) To compare measured values in HIV patients with those measured in healthy volunteers, and

3) To analyze if DTI is a valuable adjunct to conventional MRI in the early detection of HIV-associated spinal cord abnormalities.

\section{METHODS}

MR imaging of the cervical spinal cord was performed in 12 asymptomatic HIV-positive patients and in 12 healthy volunteers on 3.0 T MR scanner. All patients and volunteers underwent sagittal and axial DTI using pulsed gradient, double spin echo, echo planar imaging (TR/TE 2000/74; 128×128 matrix; 140× $140 \mathrm{~mm} \mathrm{FOV;} 10$ contiguous $4 \mathrm{~mm}$ slices; $\mathrm{b}=1000 \mathrm{~s} / \mathrm{mm} 2$ ). Average fractional anisotropy (FA) and ADC were calculated within regions of interest at bilateral anterior, lateral and posterior white matter regions of the cord. The statistical significances of the computed DTI metrics were assessed using a paired, doubletailed student $t$ test.

RESULTS

The average age of HIV-positive patients was 35 years versus 33.4 years in the control group.

No patients had abnormalities on conventional T2-weighted MR images. FA was lower in HIV-positive patients in the anterior (mean 0.66), lateral (mean 0.76), and posterior regions (mean 0.74) of the cervical spinal cord. ADC was higher compared with controls but it did not reach statistical significance.

CONCLUSION

Asymptomatic HIV + patients demonstrate changes in DTI metrics measured in the cervical spinal cord compared to healthy volunteers in the absence of spinal cord abnormalities on conventional MR sequences. These changes may represent early HIV-related changes in the spinal cord and may be markers of vacuolar myelopathy. Early identification of spinal cord abnor- 
malities in asymptomatic HIV + patients would identify patients who will benefit from antiretroviral therapy.

\section{SL05:13:5}

\section{DIFFUSION TENSOR TRACTOGRAPHY REVEALS SPINAL CORD ALTERATIONS IN PATIENTS WITH MULTIPLE SCLEROSIS}

W. Van Hecke ${ }^{1}$, A. Leemans ${ }^{2}$, J. Sijbers ${ }^{1}$, P. Parizel ${ }^{3}$, J. Van Goethem ${ }^{3}$

1 VisionLab, Department of Physics, University of Antwerp, Antwerp, Belgium; ${ }^{2}$ CUBRIC, Department of Psychology, Cardiff, Belgium; ${ }^{3}$ Department of Radiology, University of Antwerp, Antwerp, Belgium

\section{PURPOSE}

Since the spinal cord is frequently involved in Multiple Sclerosis (MS), diffusion tensor imaging(DTI) is an appropriate technique to examine white matter (WM) alterations in the spinal cord of patients with MS. However, it is known that several factors hamper a DTI study, such as movement of the patient and the relative motion of the spinal cord itself. In addition, the relatively small diameter of the spinal cord $( \pm 12 \mathrm{~mm})$ and the restricted resolution of the diffusion tensor images further impede a quantitative study. We propose the use of diffusion tensor tractography - the mathematical reconstruction of fiber bundlesto reveal WM alterations in the spinal cord of patients with MS. This approach is less sensitive to the above mentioned limitations compared with the commonly used region of interest approach. METHODS

DTI of the cervical spinal cord (C1-C5) was performed with a $1.5 \mathrm{~T}$ scanner on 21 MS patients, with a mean age of 38 years. 21 sex-and age-matched healthy volunteers were scanned. All healthy persons had a normal appearing spinal cord on conventional T2-weighted MR images. The diffusion sequence is an echoplanar sequence with $2 \times 2 \times 2 \mathrm{~mm} 3$ voxels, a b-value of $700 \mathrm{~s} / \mathrm{mm} 2$ and with with diffusion measurements in 60 directions. A fractional anisotropy (FA) threshold of 0.3 was used during tractography.

RESULTS

A mean FA, mean diffusivity (MD), first, second, and third eigenvalues of $0.522 \pm 0.004,0.00127 \pm 1.6 \mathrm{e}-5 \mathrm{~mm} 2 / \mathrm{s}, 0.002 \pm 2 \mathrm{e}$ $-5 \mathrm{~mm} 2 / \mathrm{s}, 0.00095 \pm 1.5 \mathrm{e}-5 \mathrm{~mm} 2 / \mathrm{s}, 0.0008 \pm 1.5 \mathrm{e}-5 \mathrm{~mm} 2 / \mathrm{s}$ were found for the healthy volunteers, and $0.483 \pm 0.004,0.00135 \pm 3 \mathrm{e}$ $-5 \mathrm{~mm} 2 / \mathrm{s}, \quad 0.0021 \pm 5 \mathrm{e}^{-5} \mathrm{~mm} 2 / \mathrm{s}, \quad 0.00104 \pm 1.5 \mathrm{e}^{-5} \mathrm{~mm} 2 / \mathrm{s}$, $0.00087 \pm 2.6 \mathrm{e}-5 \mathrm{~mm} 2 / \mathrm{s}$ were found for the MS patients. T-tests showed statistically significant differences for the FA $(p<0.01)$, MD $(p=0.045)$, the second $(p=0.005)$, and third eigenvalue $(p=$ 0.02 ) between the healthy and the patient group.

\section{CONCLUSION}

We present a robust segmentation approach for DT images of the spinal cord based on tractoghraphy. Statistically significant
WM alterations are found in the cervical spinal cord of MS patients.

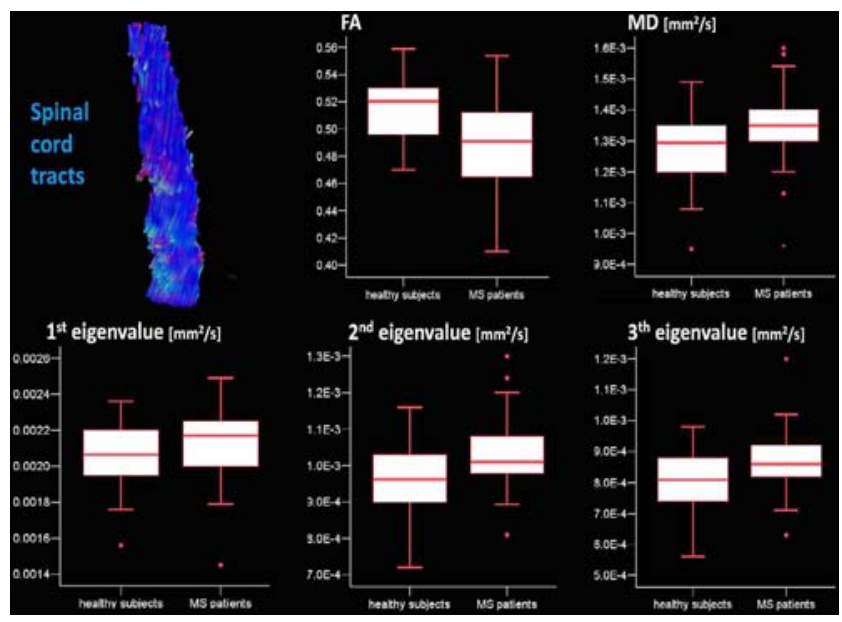

\section{SL05:13:6}

\section{THE SPINAL CORD IS AFFECTED IN ADULT-ONSET \\ AUTOSOMAL DOMINANT LEUKODYSTROPHY WITH AUTONOMIC SYMPTOMS}

J. Sundblom, A. Melberg, R. Raininko

Uppsala University, Uppsala, Sweden

\section{PURPOSE}

Brain changes in adult-onset autosomal dominant leukodystrophy (ADLD) with autonomic symptoms have been described in detail but there are no reports about the spinal cord. We describe MRI changes in the spinal cord.

\section{METHODS}

The brains and the spinal cords of six patients between the ages 43 and 63 having adult-onset ADLD with autonomic symptoms were examined with MRI. Two of them, aged 43 and 53, only had autonomic symptoms while the other patients also had pyramidal tract symptoms. The patient with the most severe symptoms (paraparesis, ataxia) was 52 years old. In addition, one asymptomatic family member, 40 years old, was examined. The spinal cords of two healthy volunteers, aged 27 and 60, were examined using the same imaging parameters to compare signal intensities. The dimensions of the spinal cords were compared to the normal values in literature.

\section{RESULTS}

All patients and the asymptomatic family member showed brain abnormalities characteristic of adult-onset ADLD with autonomic symptoms: all had involvement of the corticospinal tracts and cerebellar peduncles on T2-weighted images. Two had very limited changes, and in two other, most of the cerebral white matter was pathological.

They all showed reduced dimensions in the entire spinal cord and an increased white matter signal intensity throughout the spinal cord on 
the T2-weighted transverse images. The patient with the most severe symptoms had the largest signal intensity changes in the brain and the most severe atrophy and also grey matter changes in the spinal cord. CONCLUSIONS

Adult-onset ADLD with autonomic symptoms affects the spinal cord early, even before any symptoms. The whole spinal cord white matter is pathological at the time when the changes in the brain are very limited.

\section{SL05:13:7}

\section{FROIN'S SYNDROME. 100 YEARS ON: MAGNETIC RESONANCE IMAGING APPEARANCES}

\section{S. Mirza, W. Adams, R. Corkhill}

Department of Neuroradiology, Derriford Hospital, Plymouth, United Kingdom

Georges Froin described the condition of xanthochromic cerebrospinal fluid that rapidly clots on standing due to obstruction of the spinal subarachnoid space one hundred years ago in 1903. We report on three cases where this syndrome, caused by different pathologies, can be implied from magnetic resonance imaging.

This series comprises three patients all of whom had different pathologies that included ependymoma, prolapsed intervertebral disc and dermoid tumour, all of which caused obstruction of the spinal canal and development of Froin's syndrome. In all three cases, Magnetic Resonance imaging (MRI) was carried out. In all three cases signal return from the cerebrospinal fluid caudal to the obstructing pathology was higher than from that above the lesion especially on T2-weighted imaging. The possible patho-physiological mechanisms of this previously undescribed phenomenon, including increased levels of protein in association with other paramagnetic species as well as differential rates of cerebrospinal fluid flow in different parts of the cord are discussed.

Magnetic resonance imaging is now becoming the imaging technique of choice for evaluation of the spinal canal and it's contents and these cases show that Froin's syndrome can be a recognizable feature on MRI. We discuss the possible causes of this radiological phenomenon and postulate that this appearance on MRI may indicate a higher degree of obstruction.

\section{SL05:13:8}

\section{DIASTEMATOMYELIA: AN UNUSUAL LATE ONSET PRESENTATION WITH RADICULOPATY}

G. Callari $^{1}$, A. Arrigo ${ }^{1}$, S. Anfosso ${ }^{2}$

1 Neuroradiology of Imperia - Ponente Ligure Neurosciences Department, Imperia, Italy; ${ }^{2}$ Neurology of Imperia - Ponente Ligure Neurosciences Department, Imperia, Italy

We present the case of a 59 years old woman with a bilateral L5 radiculopaty without any neurological deficit or electrophisiolog- ical abnormality. MRI examination showed an asimptomatic diastematomyelia and L5-S1 spondilolystesis due to L5 spondilolysis responsible of L5 radiculopaty.

Diastamatomyelia can be occasionally seen in adult patients and we suggest that when this malformation is not responsable of any symtoms, any aggressive treatments are not required.

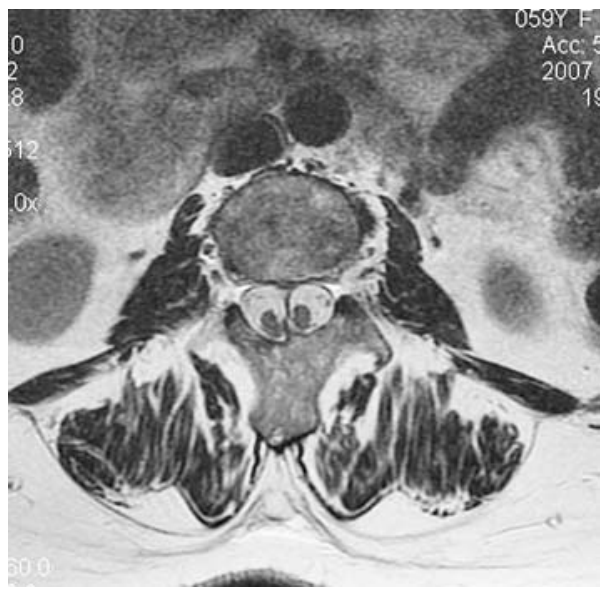

SL06

11.30-12.25 - Inflammatory and demyelinating diseases

\section{SL06:14:1}

\section{CONVENTIONAL VS NON CONVENTIONAL MRI PARAMETERS IN THE DIFFERENTIATION OF MULTIPLE SCLEROSIS CLINICAL FORMS}

M. Gavra $^{1}$, K. Voumvourakis ${ }^{2}$, M. Papathanasiou ${ }^{1}$, $\overline{\text { P. Baras }}{ }^{1}$, A. Gouliamos ${ }^{1}$

1 2NDDepartment of Radiology, University of Athens, Medical School, Attikon University Hospital, Athens, Greece; ${ }^{2}$ Department of Neurology, University of Athens, Medical School, Attikon University Hospital, Athens, Greece

\section{PURPOSE}

Different MRI parameters such as the hypointense T1 lesion load, the lesion magnetization transfer ratio (MTR) and the lesion calculated $\mathrm{T} 1$ were assessed. The above parameters were evaluated for differentiating the two main clinical forms of multiple sclerosis (MS).

MATERIALS AND METHODS

Fifty patients with clinically definite MS underwent 1,5-T MR imaging of the brain. Thirty-six of them had relapsing remitting (RRMS) and 14 had secondary progressive MS (SPMS). The Expanded Disability Status Scale (EDSS) estimated patients' disability. Total hypointense lesion load quantification was performed using an axial pre-contrast T1-weighted sequence with manual outlining and semi-automated contouring techniques. For the measurement of lesion MTR, an axial proton density-weighted 
sequence with and without application of a magnetization transfer pre-pulse was used. The mean lesion $\mathrm{T} 1$ values were measured on the T1 map with the IR/SE acquisition $(T R=1000 / 14, T E=8)$. Differences in MRI parameters between the two main clinical subgroups of MS, were assessed by Mann-Whitney test.

RESULTS

SPMS patients had a significantly higher $\mathrm{T} 1$ lesion load $(\mathrm{p}<$ $0.005)$, higher lesion T1 $(\mathrm{p}=0.005)$ and lower lesion MTR $(\mathrm{p}=$ $0.005)$ than RRMS ones. SPMS also had greater disability, than RRMS subgroup $(\mathrm{p}<0.0005)$. Of the three MRI parameters, only T1 lesion load and lesion MTR were shown to be independent variables for disability, while lesion $\mathrm{T} 1$ was not.

\section{CONCLUSION}

It could be suggested that only T1 lesion load and MTR could be used for differentiating SPMS from RRMS patients and that could potentially be used as MRI markers to monitor disease progression.

\section{SL06:14:2}

\section{HOW EARLY CAN THE DIAGNOSIS OF MULTIPLE SCLEROSIS BE ASCERTAINED WITH A SINGLE MRI SCAN?}

A. Rovira ${ }^{1}$, J. Swanton ${ }^{2}$, M. Tintore ${ }^{1}$, E. Huerga ${ }^{1}$, M. Rovaris $^{3}$, T. Korteweg ${ }^{4}$, M. Filippi ${ }^{3}$, J. Frederiksen ${ }^{5}$, A. Langkilde ${ }^{5}$, F. Barkhof ${ }^{4}$, C. Polman ${ }^{4}$, D. Miller ${ }^{2}$, X. Montalban ${ }^{1}$

${ }^{1}$ Hospital Vall d'Hebron, Barcelona, Spain; ${ }^{2}$ Institute of Neurology. University College London, London, United Kingdom;

${ }^{3}$ San Raffaele Scientific Institute, Milan, Italy;

${ }^{4}$ VU University Medical Center, Amsterdam, The Netherlands; 5 Glostrup University Hospital, Copenhaguen, Denmark

\section{PURPOSE}

According to the criteria proposed by McDonald, the diagnosis of MS in patients who present for the first time with a clinically isolated syndrome (CIS) can be achieved with a single brain MRI if obtained at least 3 months after the onset of the clinical event. The purpose of this study is to investigate the diagnostic performance of MRI obtained before the three-month interval in a large cohort of CIS patients.

\section{MATERIALS AND METHODS}

Patients who presented with a monophasic neurologic syndrome of the type seen in MS, if fulfilling the following inclusion criteria: (1) availability of two brain MR examinations, the first within three months, and the second between three and twelve months after CIS onset; (2) clinical follow-up of at least 24 months.

We compared the diagnostic properties of the two exams as regards the conversion to clinically definite MS (CDMS) according to the Poser criteria.

\section{RESULTS}

252 patients were included in this study. 66 patients presented a relapse during the first two years after symptoms onset, therefore fulfilling the diagnostic criteria for CDMS.
The comparison between the diagnostic properties of the first and second MRI for identifying patients with conversion to CDMS showed very similar results in sensitivity $(47 \%$ vs. $43 \%)$, specificity ( $88 \%$ vs., $87 \%$ ) and accuracy (77\% vs. $75 \%$ ). Assessment or non-inferiority between the two MRI scans (if $6 \%$ is considered a negligible difference) demonstrated that first and second MRIs were equivalent.

\section{CONCLUSION}

Our findings suggest that a single brain MRI may suffice to identify a subset of CIS patients with a high risk of developing CD MS, even when it is performed within the first three months after the onset of symptoms.

\section{SL06:14:3}

\section{CORTICAL INVOLVEMENT IN MULTIPLE SCLEROSIS: IMPROVED DETECTION WITH DOUBLE INVERSION RECOVERY MR SEQUENCE}

M. Gavra ${ }^{1}$, M. Papathanasiou ${ }^{1}$, K. Voumvourakis ${ }^{2}$, D. Loggitsi ${ }^{1}$, E. Staboulis ${ }^{2}$, A. Gouliamos ${ }^{1}$

1 2NDDepartment of Radiology, University of Athens, Medical School, Attikon University Hospital, Athens, Greece; ${ }^{2}$ Department of Neurology, University of Athens, Medical School, Attikon University Hospital, Athens, Greece

\section{PURPOSE}

To compare the depiction of cortical lesions by using double inversion-recovery (DIR) and fluid-attenuated inversion-recovery (FLAIR) magnetic resonance (MR) imaging in patients with multiple sclerosis (MS).

\section{MATERIALS AND METHODS}

Fourty-one patients with clinically definite MS underwent MR imaging of the brain at 1,5 T. Axial DIR and FLAIR sequences were used with $3 \mathrm{~mm}$ contiguous slices. Cortical lesions were classified according to the their location to three anatomic regions: intracortical, mixed gray-white matter and juxtacortical. The numbers of lesions per category were compared between the two sequences using the students'T test.

\section{RESULTS}

The total number of cortical lesions differed significantly between DIR and FLAIR sequences, $p<0,0001$. DIR imaging depicted an average of $7,48( \pm 8,3)$ cortical lesions per patient compared with FLAIR $6,0( \pm 7,5)$. DIR, because of high gray-white matter contrast, allowed the detection of an increased number of intracortical lesions (77) compared with FLAIR (3). DIR imaging depicted an average of $1,87( \pm 2,5)$ intracortical lesions per patient compared with FLAIR $0,07( \pm 0,26), p<0,0001$. Also, DIR enabled a better definition of mixed gray-white matter lesions (121) compared with FLAIR (57). DIR depicted an average of 2,95 $\pm 4,4$ mixed gray-white matter lesions per patient compared with FLAIR $1,39 \pm 4,3 p<0,0001$. The greatest number of lesions in FLAIR was classified as juxtacortical (186), compared with DIR (109). The average of juxtacortical lesions 
in FLAIR imaging was 4,53 $\pm 5,5$ compared with DIR 2,65 $\pm 4,05, \mathrm{p}<$ 0,0001 .

\section{CONCLUSION}

DIR sequence improves the conspicuity and detection of intracortical lesions and allows better distinction between juxtacortical and mixed gray-white matter lesions in MS patients.

\section{SL06:14:4}

\section{CORRELATION BETWEEN CORTICAL INVOLVEMENT AND CLINICAL FEATURES IN MULTIPLE SCLEROSIS PATIENTS}

\author{
M. Gavra ${ }^{1}$, M. Papathanasiou ${ }^{1}$, K. Voumvourakis ${ }^{2}$, \\ $\overline{\text { D. Kitsos }}^{2}$, E. Staboulis ${ }^{2}$, A. Gouliamos ${ }^{1}$ \\ 1 2NDDepartment of Radiology, University of Athens, Medical \\ School, Attikon University Hospital, Athens, Greece; ${ }^{2}$ Department \\ of Neurology, University of Athens, Medical School, Attikon \\ University Hospital, Athens, Greece
}

\section{PURPOSE}

To evaluate the relationship between total numbers of cortical lesions well depicted in double inversion-recovery (DIR) with disability status, disease duration, and clinical subtype in patients with multiple sclerosis (MS).

\section{MATERIALS AND METHODS}

Fourty-one MS patients were enrolled in the study and underwent MR imaging of the brain at 1,5 T. Thirteen of them had primary progressive MS (PPMS), 1 secondary progressive (SPMS), 27 relapsing remitting (RRMS). Patients' disability was estimated by the EDSS scale. Axial DIR sequence was used with $3 \mathrm{~mm}$ contiguous slices. Cortical lesions were classified according to their location to three anatomic regions: intracortical, mixed graywhite matter and juxtacortical. Correlations between disability, or disease duration, or subtype of MS and lesion scores (either total or specifically intracortical) were investigated by using Spearman correlation coefficient and students' $\mathrm{T}$ test.

\section{RESULTS}

No correlations were found between total number of cortical lesions and disability $(\mathrm{r}=0,04, \mathrm{p}=0,79)$ and disease duration $(\mathrm{r}=0,20, \mathrm{p}=0$, 21). No correlations were also found between total number of intracortical lesions and disability $(\mathrm{r}=0,04, \mathrm{p}=0,78)$ and disease duration $(r=0,01, p=0,91)$. No significant difference was found in the number of cortical lesions between the RRMS and PPMS subgroup (mean $6,77 \pm 5,96$ versus mean $9,15 \pm 12,21$ with $p=0,51$ ). CONCLUSION

Our results in accordance with other studies $(1,2)$ suggest that the number of cortical lesions, either total or specifically intracortical, detected in DIR sequence doesn't correlate with EDSS, disease duration and clinical subtype in MS patients. 1. Geurts $J$ et al in Radiology 2005; 236:254-260, 2. Bagnato F et al in AJNR Am J Neuroradiol 2006; 27:2161-67.

\section{SL06:14:5}

QUANTITATIVE CERVICAL PROTON MAGNETIC RESONANCE SPECTROSCOPY OF MULTIPLE SCLEROSIS

$\underline{\text { A. Marliani }}^{1}{ }^{\text {, V. Clementi }}{ }^{2}$, F. Salvi ${ }^{3}$, L. Albini Riccioli ${ }^{1}$, R. Agati ${ }^{1}$, M. Leonardi ${ }^{1}$

${ }^{1}$ Neuroradiology Department, Bellaria Hospital, Bologna, Italy; ${ }^{2}$ GE Healthcare, Bologna, Italy; ${ }^{3}$ Neurology Department, Bellaria Hospital, Bologna, Italy

\section{INTRODUCTION}

Frequently disability in multiple sclerosis (MS) is related to spinal cord lesions and there are few published works on spectroscopy study of cervical diseases because of the technical difficulties limiting the quality of spectroscopy data.

\section{METHODS}

A rectangular 1H-MRS VOI was prescribed along the main axis of the cord between the $\mathrm{C} 2-\mathrm{C} 3$ levels, on a plaque hyperintense on T2 weighted images, in a group of 8 MS patients using a $3 \mathrm{~T}$ wholebody system .

A PRESS and CHESS sequences were used with six saturation bands to minimize fat contamination. MRS data were analysed by the user-independent fitting routine LCModel. Relative concentrations expressed by the absolute concentration ratios of total NAA (tNAA), choline (Cho), myo-inositol (mI) and creatine plus phosphocreatine $(\mathrm{Cr})$ were calculated.

Mean, standard deviation (SD) and coefficient of variation (CV) of the main metabolite ratios were calculated. The Student's t-test were used to evaluate the difference with the healthy metabolite content previously published (same protocol, 10 healthy volunteers, mean age $35 \pm 12$ years, 6 female).

\section{RESULTS}

Despite the little group of patients the statistical analysis show a significant decrease of tNAA/Cho and an increase of $\mathrm{Cho} / \mathrm{Cr}$ contents on MS plaques respect to healthy cervical spine tissue. This trend is in accord with the metabolic abnormality already interpreted on the brain MS plaques as neuronal dysfunction and demyelination.

DISCUSSION

This preliminary result shows that the quantification cervical spectroscopy protocol optimized on healthy volunteers is reliable also for patients clinical studies. This technique offers a metabolic evaluation on the cervical cord diseases studies and in the clinical routine, as it happens in the brain. It will be interesting to apply this quantification protocol in a greater patients group

\section{SL06:14:6}

\section{FLAIR AND FLAIR-SPIR-MTC SEQUENCE IN THE DETECTION OF HIPPOCAMPAL SCLEROSIS}

C. Balassy, M. Hörmann, M. Weber, D. Prayer

General Hospital of Vienna, Medical University of Vienna, Vienna, Austria 


\section{PURPOSE}

To investigate whether FLAIR (fluid attenuated inversion recovery) sequence enhanced with SPIR (Spectral Presaturation with Inversion Recovery) and MTC (Magnetisation Transfer Contrast) improves the detection of gliotic changes associated with hippocampal sclerosis, compared to FLAIR sequence alone.

\section{METHODS}

47 consecutive patients with suspected hippocampal sclerosis $(\mathrm{M} / \mathrm{F}=$ 24/43, aged 19-60 years) underwent MR imaging on a $1.5 \mathrm{~T}$ unit. Additionally to standard imaging protocol (T2w, IR, FLAIR), FLAIRSPIR-MTC $(\mathrm{TE} / \mathrm{TR} / \mathrm{TI}=120 / 7000 / 2100$, slice thickness $=3 \mathrm{~mm} /$ $0.3 \mathrm{~mm}$ gap) was performed. FLAIR and FLAIR-SPIR-MTC sequences were read retrospectively in a random order by three radiologist, who were blinded to the diagnosis. Data were evaluated for presence of abnormality and for the quality of signal intensity changes, which was graded in scoring system of 5 points ( 1 barely visible -5 excellent delineation), irrespective of associated findings, such as atrophy or reduced grey-white differentiation on T2 or on IR sequences. Diagnosis was made by clinical and radiological examinations, and was verified by histology in 10 cases.

\section{RESULTS}

33 Patients were diagnosed with hippocampal sclerosis, 14 patients were normal. Detection rate varied not significantly in either of the two sequences in the three readers $(93.9 \%-96.9 \%, 96.9 \%-93.9 \%, 90.9 \%-$ $87.8 \%$ for FLAIR - FLAIR-SPIR-MTC).The average image quality score for FLAIR-SPIR-MTC sequence was significantly higher than for FLAIR sequence by all 3 readers (3.39/2, 3.39/2.36, 3,69/3,06, respectively). Similarly, the number false positive cases was higher with FLAIR-SPIR-MTC than with FLAIR alone (6/1, 2/1, 5/4, respectively).

\section{CONCLUSIONS}

FLAIR-SPIR-MTC seems to show higher affinity and better image quality for the detection of hippocampal sclerosis, thus suggested to be included in the imaging protocol for the workup of temporal lobe epilepsy. However, to increase specificity, the other sequences (T2w, IR) also have to be evaluated.

\section{SL06:14:7}

\section{MR FINDINGS AT CLINICAL PRESENTATION IN THIRTY- TWO ALCOHOLIC AND NON-ALCOHOLIC PATIENTS AFFECTED BY WERNICKE ENCEPHALOPATHY}

G. Zuccoli ${ }^{1}$, M. Gallucci ${ }^{2}$, C. Carollo ${ }^{3}$, J. Capellades ${ }^{4}$, L. Regnicolo 5, T. Cabada Giadas 6, J. Mandrioli ${ }^{7}$, M. Rossi ${ }^{8}$,

M. SEVERINO ${ }^{3}$, L. SANTELLI ${ }^{3}$, R. MANARA ${ }^{9}$

${ }^{1}$ Arcispedale Santa Maria Nuova, Reggio Emilia, Italy;

${ }^{2}$ University of L'aquila, L'aquila, Italy; ${ }^{3}$ University of Padova, Padova, Italy; ${ }^{4}$ University Hospital of Badalona, Badalona, Spain; ${ }^{5}$ Hospital of Ancona, Ancona, Italy;

${ }^{6}$ Hospital of Navarra, Navarra, Spain; ${ }^{7}$ Hospital of Modena, Modena, Italy; ${ }^{8}$ University of Parma, Parma, Italy;

${ }^{9}$ Neuroradiology, Padova, Italy

\section{BACKGROUND AND PURPOSE}

Wernicke encephalopathy is a severe disorder that results from vitamin B1 deficiency. The classical clinical presentation is characterized by consciousness changes, ocular abnormalities, and ataxia. This study was undertaken to analyze and compare findings on MR imaging and neurologic symptoms at clinical presentations of patients with Wernicke encephalopathy with and without a history of alcohol abuse.

MATERIALS AND METHODS

A multicenter, retrospective study reviewed MR brain imaging findings, clinical histories, and neurological presentations of 32 patients (17 female, 15 male) affected by Wernicke encephalopathy between 1999 and 2007. The age range was 6-86 year.

\section{RESULTS}

Fifty-Six percent of the patients had no history of alcohol abuse, and $44 \%$ had a history of alcohol abuse. Eighty-One percent showed consciousness changes, $77 \%$ had ocular symptoms, and $59 \%$ had ataxia. Only $46 \%$ of the patients showed the classic triad of the disease at clinical presentation. At MR examination, $84 \%$ of the patients showed symmetric lesions in the medial thalami and the periventricular region of the third ventricle, $66 \%$ in the periaqueductal area, $50 \%$ in the mamillary bodies, $31 \%$ in the tectal plate, and $6 \%$ in the dorsal medulla. In singular cases were involved the cerebellum and the hypothalamus. Contrast enhancement of the mamillary bodies was correlated with the alcohol abuse group. Alterations in the medial thalami and the periventricular region of the third ventricle were statistically correlated with no history of alcohol abuse.

\section{CONCLUSIONS}

Our study confirms the usefulness of MR in reaching a prompt diagnosis and treatment of Wernicke encephalopathy to avoid brain tissue damage, highlighting neuroradiological patterns of presentation in alcoholic and non-alcoholic patients.

\section{SL07}

\subsection{5-16.30 - Session XV - Pediatrics: technique and congenital abnormalities}

\section{SL07:15:1}

\section{T MRI CONTRAST ENHANCED BRAIN PATHOLOGY: GRADIENT ECHO T1 OR SPIN ECHO T1?}

R. Zimmerman, L. Bilaniuk, A. Pollock, E. Schwartz,

T. Feygin, D. Zarnow

The Children's Hospital of Philadelphia, Philadelphia, USA

\section{PURPOSE}

To evaluate the utility of two different $\mathrm{T} 1$ techniques in performing post gadolinium-enhanced imaging at $3 \mathrm{~T}$. 3D gradient 
echo T1 shows superior gray-white differentiation at $3 \mathrm{~T}$ compared with spin echo T1 images. The question is whether you lose information regarding contrast enhancement with gradient echo T1.

\section{MATERIAL \& METHODS}

Forty infants and children with abnormalities of blood brain barrier due to tumor, infection, infarction or inflammation were examined at $3 \mathrm{~T}$ post-gadolinium injection by both a 3D T1 gradient echo sequence and a fat-saturated spin-echo sequence.

RESULTS

Almost all intraparenchymal tumor masses showed similar contrast enhancement by both methods. However, more subtle enhancement at sites of inflammation (multiple sclerosis) and within the subarachnoid space (tumor dissemination) were better seen or only seen on spin echo images.

CONCLUSION

We continue to do both sets of $\mathrm{T} 1$ images post contrast at 3T.

\section{SL07:15:2}

\section{CONGENITAL BRAIN MALFORMATIONS OF CORTICAL DEVELOPMENT}

Z. Merhemic, F. Gavrankapetanovic, Z. Kadenic, M. Niksic, G. Sulejmanpasic Clinical Centre University Sarajevo, Sarajevo, Bosnia Herzegovina

\section{PURPOSE}

Complex processese of neuronal proliferation, differentiation, migration, and organization occur between 2 and 5 months of gestation. Disturption in the normal process results in spectrum of brain malformations.

The aim of this study is to present several types of malformations depending on the timing and severity of the arrest of cortical development.

\section{MATERIALS AND METHODS}

Twenty six patients with malformation of cortical development, 12 female and 14 male, range age 0 to 22 years, mean age 7,3 years; were egzamined on MR unit using standard sequences (T1,PD,T2, FLAIR WI), usualy in all planes.

RESULTS

Six patients had lissencephaly, 4 combined with laminar heterotopia. Three polymicrogyria; eight schisencephaly, 6 unilateral, and 2 billateral, 2 open lip, other close lip. Three patients had gray matter heterothopia, 2 nodular and 1 subependimal. Seven patients had unilateral megalencephaly.

\section{CONCLUSION}

MR imaging has proved to be a diagnostic tool of major importance in patients with congenital brain malformations of cortical development. The exellent gray/white matter differeren- tiation and multiplanar imaging capabilities of MR allow a systematic analysis of the brain.

\section{SL07:15:3}

\section{CALLOSAL DYSGENESIA DUE TO HEMIMEGALENCEPHALY IN PHAKOMATOSIS}

P. Ventura ${ }^{1}$, M. Pulito ${ }^{1}$, A. Furlano ${ }^{2}$, D. Perito ${ }^{2}$, G. Scarpelli ${ }^{2}$, G. Riccipetitoni ${ }^{3}$,W. Auteri ${ }^{1}$

${ }^{1}$ Unit of Neuroradiology Annunziata Hospital, Cosenza, Italy;

${ }^{2}$ Unit of Neonatal Care Annunziata Hospital, Cosenza, Italy;

${ }^{3}$ Unit of Paediatric Surgery Annunziata Hospital, Cosenza, Italy

\section{PURPOSE}

The corpus callosum develops from anterior to posterior. We report a patient with callosal dysgenesis in which only posterior body and splenium were present related to hemimegalencephaly. We suppose this condition interfered with callosal development. The association of hemigalencephaly, cutaneous anomalies and cerebral vascular displasia suggested a phakomatosis.

Key words: Corpus callosum dysgenesis Hemimegalencephaly Phakomatosis

\section{CASE REPORT}

We present a 10 weeks old female, born term delivered. The child showed forehead hemangioma; by surpise, no seizure disorder was observed.

MRI showed hamartomatous appearence of right fronto-parietal region with dysplastic cortex, broad gyri and shallow sulci. Midline shift was present.

Cortical infolding was bordered by thickned cortex, as well as gyrus cinguli. Ipsilateral ventricle was enlarged with frontal horn pointing superiorly and anteriorly. The posterior body and splenium of the corpus callosum were present, while anterior body, genu and rostrum were not. Controlateral perirolandic region showed abnormal cortical gyral pattern and horizontal fashion of rolandic scissure. Right internal carotid artery had a dolicomegalic appearance.

No visceral organs involvement was observed.

\section{DISCUSSION}

We report a case of callosal dysgenesis excepting the normal "front to back" callosal development related to focal hemimegalencephaly. As our known this association was never described before. We suppose hemimegalencephaly apparently interfered with callosal development before 20 gestational weeks; commissural plate might not be the only region where the corpus callosum starts to develop in some congenital brain malformations.

In our patient hemimegalencephaly, cutaneous anomalies and cerebral vascular displasia suggested a phakomatosis.

The present case didn't meet full criteria for any of the major neurocutaneous disorders. Relating to vascular dysplasia of the 
affected hemisphere a vascular etiology for this condition was supposed.

\section{SL07:15:4}

\section{DIFFUSION TENSOR FIBER TRACKING REVEALS PROBST BUNDLES IN PATIENTS WITH AGENESIS OF THE CORPUS CALLOSUM}

W. Van Hecke ${ }^{1}$, A. Leemans ${ }^{2}$, N. De Brabander ${ }^{3}$, A. Laridon ${ }^{3}$, K. Claeys ${ }^{3}$, B. Ceulemans ${ }^{3}$, J. Van Goethem ${ }^{4}$, P. Parizel ${ }^{4}$, J. Sijbers ${ }^{1}$

1 VisionLab, Department of Physics, University of Antwerp, Antwerp, Belgium; ${ }^{2}$ CUBRIC, Department of Psychology, Cardiff, United Kingdom; ${ }^{3}$ Department of Pediatric Neurology, University of Antwerp, Antwerp, Belgium; ${ }^{4}$ Department of Radiology, University of Antwerp, Antwerp, Belgium

\section{INTRODUCTION}

Diffusion tensor imaging(DTI) is a recently developed technique that measures the diffusion of water molecules in tissues. From DTI data, diffusion tensor tractography(DTT) can reconstruct the white matter(WM) fiber architecture. The objective of this study is to demonstrate the potential of DTT to study the structural anomalies of WM fiber tracts in patients with agenesis of the corpus callosum(CC). An interesting feature in these patients is the presence of anteroposteriorly oriented WM fiber tracts which run parallel to the interhemispheric fissure, medial to the lateral ventricles. These tracts are known as the Probst bundles (PB). METHODS

5 patients with agenesis of the corpus callosum were studied ( 2 months -2 years old). All patients were examined in a 1.5 T MR system with $40 \mathrm{mT} / \mathrm{m}$ gradients. The diffusion sequence is an echoplanar sequence with $2 \times 2 \times 2 \mathrm{~mm} 3$ voxels, a b-value of $700 \mathrm{~s} /$ $\mathrm{mm} 2$ and with diffusion measurements in 60 directions.

\section{RESULTS}

Convincing fiber tracking data sets were obtained in all cases, indicating total absence of the $\mathrm{CC}$ and the presence of PB. We found a significant variability in the appearance and architecture of the PB between the subjects. Other WM tracts differed not significantly from normal brain tissue.

\section{CONCLUSION}

With DTT, the abnormal organization of WM bundles in the brain of patients with $\mathrm{CC}$ agenesis can be shown. This techniques is well qualified for an examination of such a structural anomaly, since it specifically measures and visualizes the diffusion process in tissues, which can be related to the WM fiber architecture. Our data correlate perfectly with anatomopathological findings. The variations in fiber architecture of the Probst bundles can possibly be attributed to the brain plasticity. Analogous to the development of the $\mathrm{CC}$, an increase of the fractional anisotropy and the number of fiber tracts in the Probst bundles was demonstrated with age.

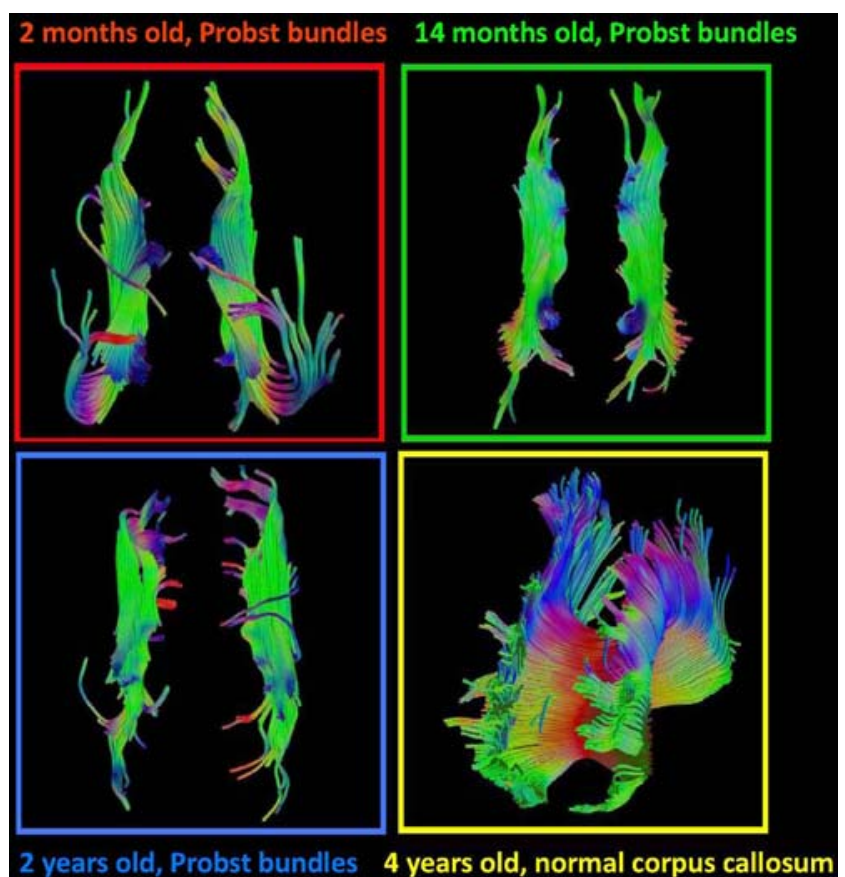

\section{SL07:15:5}

\section{A SPECTRUM OF UNUSUAL NEUROIMAGING FINDINGS IN SUSPECTED STURGE WEBER SYNDROME}

\section{Adams, S. Aylett, K. Chong}

Great Ormond Street Hospital, London, United Kingdom

\section{PURPOSE}

The early identification of Sturge Weber syndrome in patients with port wine stains is important both for prognostic purposes and because the prompt treatment of seizures may affect long term outcome. In this study we present a spectrum of unusual imaging findings in patients with suspected Sturge Weber syndrome.

\section{METHODS}

A retrospective review of the neuroimaging was carried out for all patients referred to Great Ormond Street hospital for investigation of facial port wine stain and suspected Sturge Weber syndrome between January 2003 and March 2007.

\section{RESULTS}

8 patients exhibited imaging features not considered conventional for Sturge Weber syndrome. In 5 patients this was manifest as pial enhancement in an unusual anatomical location with 2 cases of combined supra and infratentorial enhancement and 3 cases of purely infratentorial enhancement, 2 of which had involvement of the brainstem only. One patient exhibited progressive macrocephaly with a unilateral enlarged globe, anomalous venous drainage and no pial angioma; one patient showed progressive parenchymal atrophy and calcification without evidence of pial angioma; and one patient exhibited grossly abnormal venous drainage with florid developmental venous anomalies.

\section{CONCLUSIONS}

We have identified several atypical imaging findings in patients with clinically suspected Sturge Weber syndrome. The 5 cases with 
infratentorial pial enhancement are likely to represent an extension of the conventional spectrum of pial angioma formation. The three remaining cases may represent different end points of the same pathological entity underlying more usual imaging manifestations, possibly reflecting impairment of venous drainage. Radiologists should be aware of these atypical features when interpreting the imaging of children with facial port wine stain. Whether these imaging appearances are associated with seizures or adverse outcome is the subject of long term follow up.

\section{SL07:15:6}

\section{THE MRI IMAGE IN THE ASPERGER SYNDROME}

$\frac{\text { J. Walecki }}{\text { B. Kubas }}{ }^{1}$, A. Sybilski ${ }^{2}$, K. Kaminska ${ }^{2}$, M. Szczerbicki ${ }^{2}$,

${ }^{1}$ Polish Academy of Sciences Medical Research Center, Warsaw, Poland; ${ }^{2}$ Central Clinical Hospital of the Ministry of Internal Affairs and Administration, Warsaw, Poland

\section{PURPOSE}

Asperger Syndrome is a complex developmental disorder which demonstrates itself with social dysfunctions and lack of interests, without cognitive or speech impairment.

In children with the Asperger syndrome the growth of brain is accelerated in the first few years and retarded afterwards. The authors focus on the assessment of structural changes in children of the higher age group by analyzing the structures strategic for the Asperger syndrome.

MATERIAL

The MRI examinations were performed to 21 children with the Asperger syndrome (aged 7-15)

All patients matched the clinical criteria of the disease according to ICD-10, the clinical observation was not shorter than 3 years. Control group included MR of brain in 16 patients aged 5-15 without clinical symptoms.

\section{METHOD}

The MRI were performed using 1.5 T scanner, TSE (with T2 inversion) and FLAIR sequences and planes- frontal and transverse.

Converted MR images were imported to MRIcro 1.4 software in order to analyze and measure the volume of the following structures: caudate and lenticular nuclei, and the area of frontal cortex. The fluid/cerebral index was also calculated using the area of lateral ventricles on their widest level.

\section{Results}

In 11 children $(45 \%)$ we found statistically significant $(\mathrm{p}<0.05)$ decrease area of cortex in the prefrontal area, in 13 children $(60 \%)$ an in significant increase in the volume of caudate and lenticular nuclei. In 15 patients $(70 \%)$ the ventricle-cerebral index showed slightly widening of ventricular system concerns both lateral verticles comparison to the control group.

\section{PRELIMINARY CONCLUSIONS}

The structural MR image corroborates the thesis of a more frequent incidence of structural lesions in children with the Asperger syndrome.
Decreased area of frontal cortex and increased the volume of caudate and lenticular nuclei as well, seems to be structural pattern for Asperger disease in middle - age children with more than 5 years disease.

\section{SL07:15:7}

\section{CAVERNOUS MALFORMATIONS OF THE CNS: EPIDEMIOLOGY AND CLINICAL FINDINGS IN THE UNIVERSITY HOSPITAL BERN; A TEN-YEARS EXPERIENCE}

\author{
M. El-Koussy ${ }^{1}$, F. Stepper ${ }^{2}$, A. Spreng ${ }^{1}$, \\ L. Remonda $^{1}$, C. Ozdoba ${ }^{1}$, M. Sturzenegger ${ }^{2}$, G. Schroth ${ }^{1}$ \\ ${ }^{1}$ Dept. Neuroradiology, University Bern, Bern, Switzerland; \\ ${ }^{2}$ Dept. Neurology, University Bern, Bern, Switzerland
}

\section{INTRODUCTION}

Symptomatic cavernous malformations (CM) can adversely affect a subject's quality of life. No studies on the epidemiology of CM of the central nervous system (CNS) and related symptoms exits in current literature.

\section{METHODS}

We analyzed the patients who were admitted to the University Hospital in Bern and diagnosed as having an CM related to the CNS. The prevalence, age and gender distribution, the symptomatology pattern and the frequency of the hereditary form was to be analyzed.

RESULTS

In the period from 1985-2004, 347 (average age 42; 41.1\% females) cases were to our institution. The CM was documented by computed tomography or magnetic resonance imaging.

The study cohort included 87 asymptomatic and 260 symptomatic cases. Till 1995, there was progressive increase in symptomatic and asymptomatic cases, the latter had a 6-year delay. The number of the cases since 1995 remained relatively constant with 13-28 symptomatic and 5-14 asymptomatic cases/year.

Symptomatic CMs were diagnosed on average at the age of 36 (range: newborn-79 y). At the time of the diagnosis asymptomatic patients were $6.3 \mathrm{y}$ older than symptomatic cases. Mean age at symptom begin for hemorrhage, epilepsy and mass effect was 38.9, 29.8 and $36.6 y$ respectively.

A total of 728 lesions were detected with $69 \%$ of lesions having a supra-tentorial localization. Infratentorial, spinal and orbital CMs affected $22.2 \%, 3 \%$ and $1.5 \%$ of patients respectively. Multiple lesions were detected in $18.9 \%$ of cases. The lesions' sizes (range: $0.2-8 \mathrm{~cm}$ ) were higher for the symptomatic $(1.75 \mathrm{~cm}, \mathrm{n}=164)$ than asymptomatic cases $(0.91 \mathrm{~cm}, \mathrm{n}=64)$.

Twelve families had 29 members ( $8.4 \%$ of cohort) with autosomal dominant heredity could be distinguished. Eighteen (62\%) of these cases revealed multiple CMs. Additional 3 cases $(0.9 \%)$ could be related to previous radiation therapy.

\section{CONCLUSION}

Epidemiology of CMs follows the general trend worldwide, with obvious hereditary variant present in and around Bern, Switzerland. The value of screening remains doubtful. 

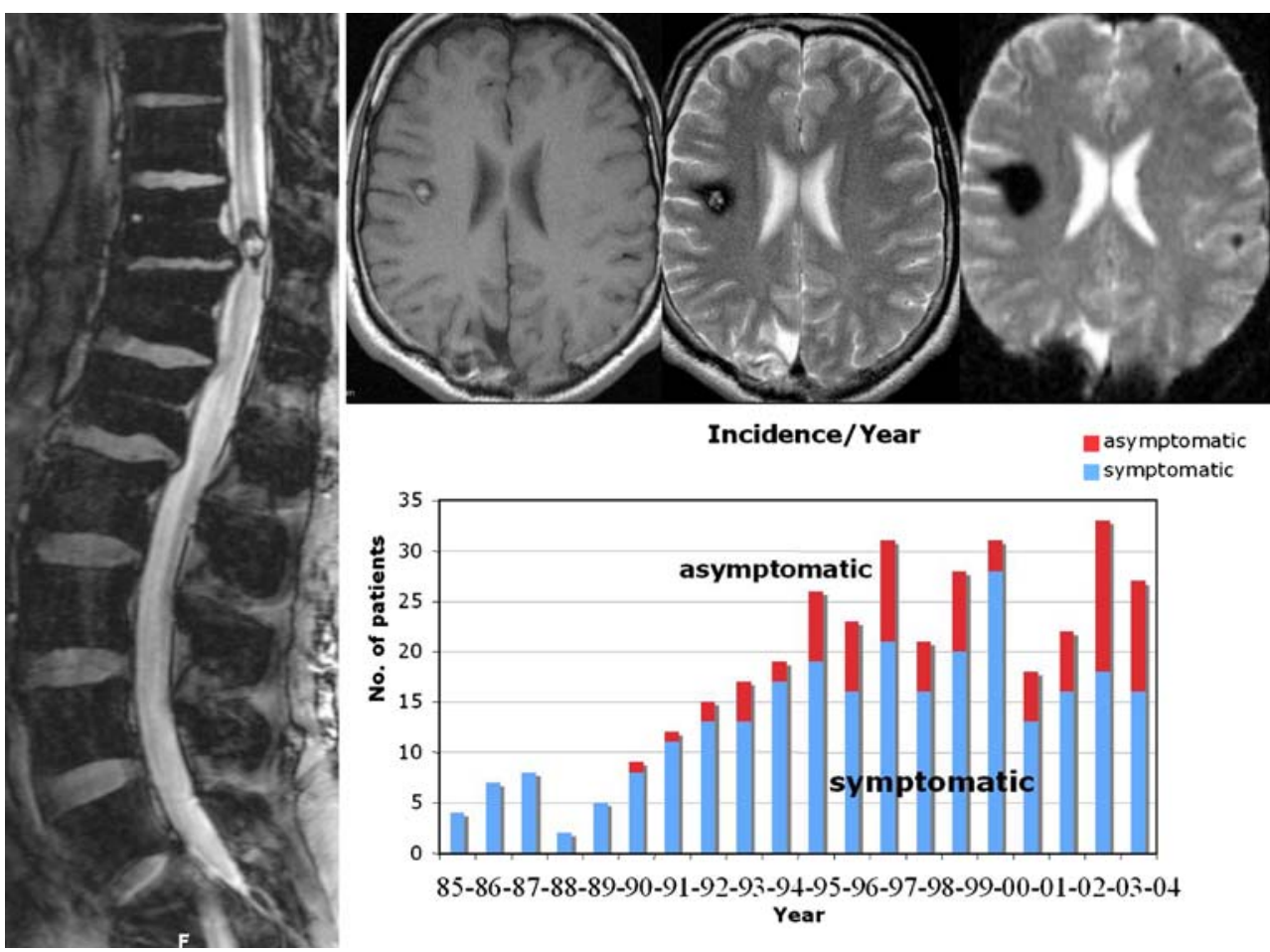

\section{SL07:15:8}

\section{ARNOLD-CHIARI TYPE I: TWO UNUSUAL CASES WITH CLINICAL AND RADIOLOGICAL CORRELATION}

R. Cerini, N. Faccioli, M. Barillari, F. Vecchiato,

R. Pozzi Mucelli

Istituto di Radiologia, Policlinico G.B. Rossi, Università di Verona, Verona, Italy

\section{PURPOSE}

In this report we show two cases of Arnold-Chiari type I in pediatrics Patients, in which the first and the second Magnetic Resonance (MR) exam showed a modification respect to the first exam.

\section{METHODS}

Two boys underwent two different Magnetic Resonance (MR) Imaging examinations with a distance of $4-5$ years; the first boy was 18 months old boy, the second 4 years old: each had a delay in the motor development.

\section{RESULTS}

For the first boy, the first examination was negative. At the second investigation, cause to atassia and brainstem hypotonia, on T1weighted images was evident a descent of cerebellar tonsils for $1,8 \mathrm{~cm}$ into the foramen occipitale magnum with syringomyelia, and no other associated findings. For the second boy, on the first examination, on $\mathrm{T} 1$ weighted images was evident a descent of the cerebellar tonsils into the foramen occipitale magnum for $1,5 \mathrm{~cm}$, and no other associated findings. The second examination, at 6 years old, was performed after a hypotensive episode, and showed the complete resolution of the malformative aspect with normal position of the cerebellar tonsils. In the first case here reported we performed a diagnosis of acquired Arnold-Chiari type I, but in the second case, the previously diagnosed Arnold-Chiari type I, seem to be vanished. CONCLUSION

These two cases demonstrates that Arnold-Chiari type I malformation may evolve with progression or regression. The eventual surgical approach in Patients have to be clinically confirmed. Patients with neurological symptoms but no imaging findings have to be followed up.

\section{SL07:15:9}

\section{MRI SPINE EVALUATION IN CHILDREN WITH ANORECTAL MALFORMATIONS}

$\underline{\text { M. Severino }}^{1}$, R. Manara ${ }^{1}$, M. Calderone ${ }^{1}$, P. Midrio ${ }^{2}$, $\overline{\text { S. Giuliani }^{2}}$, C. Dalle Nogare ${ }^{2}$, R. Mardari ${ }^{1}$, L. Santelli ${ }^{1}$, V. Citton ${ }^{1}$, C. Carollo ${ }^{1}$

${ }^{1}$ Neuroradiologic Unit - University of Padua, Padova, Italy;

${ }^{2}$ Paediatric Surgery - University of Padua, Padova, Italy

\section{BACKGROUND}

Anorectal anomalies (ARA) and spinal dysraphism (SD) is a wellknown association, although most of previous reports do not clearly define the type of SD.

Purpose: to assess prevalence and type of SD in ARA patients according to the new Tortori-Donati classification and to study the association between ARA and spinal epidermoid cysts. 


\section{METHODS}

Among 71 ARA patients referring to our Hospital since 1999, 47 patients (28 males, mean age 2,5 years, range 1 month-10 years), underwent spinal MR. 43 MR studies were available for evaluation and were reviewed by 3 neuroradiologist blinded to the clinical severity. In case of discordance, the images were jointly discussed. All studies were performed with 1.0-T magnet (Marconi Picker Polaris). Axial and sagittal spin-echo T1weighted images, T2-weighted images were obtained; in some cases coronal T1 or T2 images were also performed. Moreover, diffusion-weighted (DWI) echo-planar images were produced and corresponding apparent diffusion coefficient (ADC) values were calculated whenever we found an intraspinal fluid-like lesion.

RESULTS

Among our ARA patients SD was observed in 30 cases $(69,7 \%)$, 17 presented a tethered cord. Sixteen patients $(37,2 \%)$ had a closed SD: 1 intradural lipoma, 2 tight filum terminale and 13 filar lipoma; dermal sinus was observed in 2 patients with filar lipoma. Twelve patients $(27,9 \%)$ had a complex SD: 4 caudal agenesis type I and 8 caudal agenesis type II (the tethered cord was due to intradural lipoma in 2 cases and to filar lipoma in 6 cases; a tight filum terminale was present in 2 patients with filar lipoma). Moreover 2 patients had a complex SD associated to a closed SD (caudal agenesis type II with lipomyelomeningocele and lipomyelocele). Twenty-four patients $(55,8 \%)$ showed minor vertebral anomalies; three of them had no other SD. A presacral mass was detected in 2 cases that prompted further paediatric surgical evaluation. Two intraspinal fluid-like lesions were found and showed an increase in size on follow-up MR scans: one lesion resulted to be a sacral arachnoid cyst with bone remodelling while the other lesion appeared hyperintense on DWI revealing to be an epidermoid cyst.

CONCLUSIONS

Closed and complex SD are frequent in ARA patients and a complete spine MRI study is mandatory. Analysis of the results may be difficult and requires neuroradiologic expertise to avoid either under or overestimation of the malformations. DWI should be performed in order to recognise epidermoid cyst in ARA patients with fluid-like lesions.

\section{Levante - Ponente Room}

\section{LP01}

09.00-9.55 - Pediatrics: fetal and neonatal imaging

\section{LP01:16:1}

\section{THE VOLUME OF THE FETAL TEMPORAL LOBES WITH CMV INFECTION}

C. Hoffmann, R. Grossman, A. Biegon

Sheba Medical Center, Ramat-Gan, Israel

\section{PURPOSE}

to compare the volume of the fetal temporal lobes with MRI in cases proven to have an infection with CMV and to compare the results to normal fetal brain without $\mathrm{CMV}$ infection.

\section{METHODS}

The study group contained eight infected fetuses with CMV and 22 controls with gestational age 30-38 weeks who underwent MRI scan in our MRI unit between 2002 and 2006. The patients and controls were divided for two groups: 30-34 weeks gestational age and 34 38 weeks of gestational age. The data were transformed from DIACOM to TIFF format and analyzed with NIH Image (SCION) software. The temporal lobes were measured from the coronal plane slices, and the parenchymal volume, as well as the ventricular and hemispheric volumes, were calculated.

RESULTS

The volume of the temporal lobes was insignificantly lower in the first group: $26,528 \mathrm{~mm} 3$ compared with $32,225 \mathrm{~mm} 3$ the volume of the second group, of gestational age 34-38 weeks were lower significantly $(\mathrm{p}<0 / 05): 37,695 \mathrm{~mm} 3$ as compared to $51,401 \mathrm{~mm}$. CONCLUSIONS

The volume of the temporal lobes was significantly lower in the fetuses with proven CMV infection in the second part of the third trimester. The difference with the first group can be due to the slow change in the volume after the infection that can be due to a slower development of the brain rather than destruction of the formed brain. A follow-up of the born children should be done to determine the importance of this volumetric change

\section{LP01:16:2}

\section{MRI FINDINGS IN FETUSES WITH NEURAL TUBE DEFECTS WITHOUT HINDBRAIN HERNIATION}

\author{
L. Bilaniuk, E. Schwartz, R. Zimmerman, T. Feygin, \\ D. Zarnow, A. Pollock \\ The Children's Hospital of Philadelphia, Philadelphia, USA
}

\section{PURPOSE}

To characterize fetal MRI findings in a group of fetuses with lumbosacral spinal defects without associated hindbrain herniation and to correlate the MRI results with clinical and surgical findings. METHODS

During a 10-year period of fetal MR imaging at a large fetal medicine treatment center, there were 277 fetuses diagnosed with a lumbosacral or sacral defect. Twenty-six of these did not have associated hindbrain herniation. The gestational age of the fetuses ranged from 20 to 35 weeks, with an average of 24 weeks. MRIs, clinical charts, and surgical notes were reviewed from the group without Chiari II changes.

\section{RESULTS}

The posterior spinal defects were located in lumbosacral or sacral region. The sac size ranged from small to very large, and in all cases the sac wall was noted to be thicker than usually seen with myelomeningoceles. The intracranial spaces were normal in size or slightly to moderately diminished. Twenty-one had normal ventricular size. Of 17 that were tested for maternal serum and/or amniotic 
Alpha-fetoprotein and Acetylcholinesterase, 11 were normal and 6 abnormal indicating open neural tube defect. Follow-up diagnoses were available in 21: 11 myelomeningoceles, 8 myelocystoceles and 2 lipomyelomeningoceles.

CONCLUSIONS

Infrequently myelomeningoceles may not be associated with hindbrain herniation and may mimic a skin-covered lesion such as a terminal myelocystocele. The diagnosis of terminal myelocystocele is supported if other abnormalities such as omphalocele, cloacal exstrophy, imperforate anus and spinal anomalies coexist.

\section{LP01:16:3}

\section{ASSESSMENT OF THE POSTERIOR FOSSA AND VENTRICLES IN FETUSES WITH OPEN SPINAL DYSRAPHISM (OSD) BY INTERVAL IN UTERO MR IMAGING (IUMRI)}

R. Batty, E. Whitby, P. Griffiths

Academic Unit of Radiology, Floor C,

Royal Hallamshire Hospital, Glossop Road, Sheffield, United Kingdom

\section{PURPOSE}

To assess bony posterior fossa (BPF) and cerebellar vermis (CV) growth; ventricular size; OSD area and sac volume; in fetuses with OSD.

\section{METHOD}

A prospective study of 13 consecutive fetuses with lumbosacral OSD, imaged twice during pregnancy (first-iuMRI: 18-24 weeksgestation; second-iuMRI: 29-34 weeks-gestation). 13 normal fetuses were also studied. CV and BPF surface area were measured on the midline sagittal image. Trigone transverse diameter was measured in the axial plane. Orthogonal dimensions of the OSD and sac were measured.

\section{RESULTS}

10/13 fetuses had a Chiari-II-deformity, all detectable on firstiuMRI. BPF surface area was significantly smaller in fetuses with Chiari-II-deformity $(\mathrm{t}=7.77$ alpha $=0.05)$ but was normal in the non-Chiari-II-deformity group $(\mathrm{t}=0.78$ alpha $=0.05)$. BPF surface area increased by an estimated $24 \mathrm{~mm}$-squared/week in the ChiariII-deformity group; $42 \mathrm{~mm}$-squared/week in the non-Chiari-IIdeformity group and $45 \mathrm{~mm}$-squared/week in normal cases. There was no significant difference in CV area and growth between OSD patients and normal. Trigone diameter was greater than or equal to $10 \mathrm{~mm}$ in the Chiari-II-deformity group in $8 / 10$ on first-iuMRI and $10 / 10$ on second-iuMRI. In the non-Chiari-II-deformity group trigone diameter was less than $10 \mathrm{~mm}$. Mean spinal defect area measured $136 \mathrm{~mm}$-squared (range 5-511) in the Chiari-II-deformity group and $32 \mathrm{~mm}$-squared (range 7-87) in the non-Chiari-II-deformity group. Mean OSD sac volume measured 9907 mm-cubed (range 340 109369) in the Chiari-II-deformity group and 29194 mm-cubed (range 425-162226) in the non-Chiari-II-deformity group.

CONCLUSION

OSD are frequently but not always associated with Chiari-II-deformity. BPF size is small and growth rate reduced in Chiari-IIdeformity but $\mathrm{CV}$ size and growth rate is normal. Ventriculomegaly is detectable early and is progressive in Chiari-II-deformity. OSD sac volume and defect-area cannot predict Chiari-II-deformity development. This study was financially supported by the Association for Spina Bifida and Hydrocephalus (UK).

\section{LP01:16:4}

\section{FIBER TRACKING OF NORMAL AND ABNORMAL BRAIN DEVELOPMENT IN UTERO}

G. Kasprian, P. Brugger, F. Stuhr, C. Lindner, D. Prayer

Medical University of Vienna, Vienna, Austria

\section{PURPOSE}

To assess the potential of Diffusion tensor imaging (DTI) and tractography in the visualization of normal and abnormal brain connectivity in living human fetuses in utero.

\section{METHODS}

Magnetic resonance imaging of 21 unsedated fetuses with normal brains, and 4 fetuses with brain pathologies, consisting of malformations (2) and acquired pathologies (2), aged 22-37 gestational weeks, was performed including T2-weighted (w) fast spin-echo sequences, diffusionw sequences, and $\mathrm{T} 1 \mathrm{w}$ sequences, using a $1.5 \mathrm{~T}$ superconducting unit. DTI (32 directions, FOV $230 \mathrm{~mm}$, slice thickness $5 \mathrm{~mm}$, acquisition time $1.46 \mathrm{~min}$ ) was performed in corresponding axial and/or coronar planes. For tractography of non-myelinated motor, sensory and callosal fiber pathways a multi region of interest analysis was done using a Philips Intera release 11 workstation.

\section{RESULTS}

In $50 \%$ of examined fetuses fiber tracking of all major (corticospinal, thalamocortical, frontopontine and callosal) pathways was successful. In $75 \%$ at least one of these tracts could be visualized. In vivo anatomy of depicted sensomotor tracts corresponded to the transient patterns of connectivity, known from histology. Genu and splenium fibers of the corpus callosum were visible at $22 \mathrm{GW}$ (Figure A). In abnormal fetal brains disrupted and aberrant fibers (bundle of Probst, Figure B) could be detected before their appearance on structural images.

\section{CONCLUSIONS}

The fiber anatomy of the normal fetal brain can be studied by DTI in utero. Furthermore, the visualization of abnormal fiber connectivity in fetal brain pathologies is possible antenatally. 

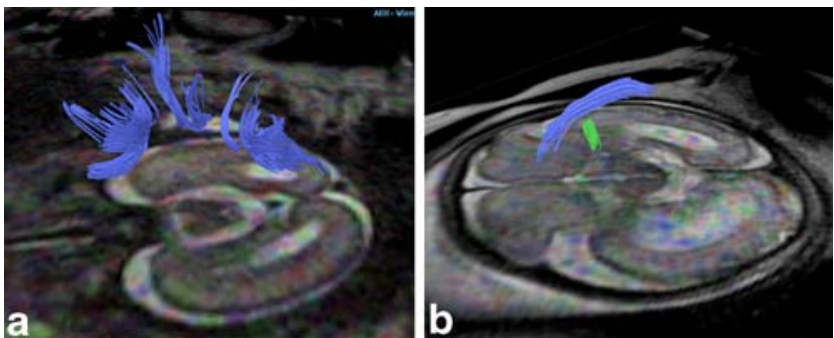

\section{LP01:16:5}

\section{NOVEL APPLICATION OF QUALITATIVE FUNCTIONAL CINE MRI TECHNIQUE IN PRENATAL ASSESSMENT}

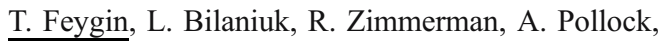
K. Shekdar, A. Voorhees

The Children's Hospital of Phialadelphia, Phialadelphia, USA

\section{PURPOSE}

To determine the role of real time cine Magnetic Resonance Imaging (MRI) in evaluating functional impairment of the fetal central neural system occurring in various pathologic entities.

\section{MATERIALS AND METHODS}

Fetal MRI studies of 32 patients, utilizing TrueFISP cine technique, were retrospectively reviewed. The fetal age ranged from 19 to 32 weeks. Patients were evaluated for either CNS or somatic pathology. In addition to routine morphologic examination of the fetus with conventional imaging, several dynamic processes in the fetus were evaluated using cine MRI. Physical parameters, such as CSF flow at the cranio-cervical junction and aqueduct of Sylvius, as well as fetal swallowing, were inspected and analyzed using signal-time curves. All studies were performed on 1.5 Tesla Siemens MAGNETOM Vision and Avanto Systems. RESULTS

All fetal patients were divided into two groups: one with known cerebral or head and neck pathology and the other with no identifiable CNS disease. The first group consisted of fetuses with Chiari II malformation, Dandy-Walker malformation, hydrocephalus, and neck masses. Four of five fetuses with Chiari II malformation were seen to have impaired CSF flow. Four fetuses with hydrocephalus were seen to have turbulent/irregular flow through the aqueduct of Sylvius. Of the five fetuses identified with a neck mass, one had preserved swallowing with normal epiglottis motion, while the other four failed to demonstrate normal swallowing. The second group included fetuses with somatic pathology. Normal patterns of CSF flow as well as all three phases of swallowing, including oral phase, pharyngeal phase and esophageal phase, were observed in the second group. CONCLUSION

The cine MR technique appears to be a valuable tool in prenatal assessment of functional impairment in several types of fetal pathology, affecting immediate clinical decision-making and postnatal management.

\section{LP01:16:6}

\section{CHROMOSOMAL ANOMALIES AND PRENATAL MRI FINDINGS}

C. Parazzini ${ }^{1}$, A. Righini ${ }^{1}$, L. Spaccini ${ }^{1}$, F. Lalatta ${ }^{2}$, M. Rustico $^{1}$, F. Triulzi ${ }^{1}$

${ }^{1}$ Ospedale dei Bambini V Buzzi Dpt Radiologia

e Neuroradiologia, Milano, Italy; ${ }^{2}$ Irccs Policlinico, Milano, Italy

\section{PURPOSE}

The aim of the study was to find associations between brain anomalies on fetal MR imaging and chromosomal mutations in order to evaluate the role and diagnostic value of prenatal MRI in this conditions.

\section{METHODS}

From a data base of about 1050 prenatal fetal MR imaging studies performed in our Institution between 2001 and 2006, we retrospectively selected 26 cases of fetal brain MR imaging which karyotipe, performed during the pregnancy or after birth or abortion, revealed structural or numerical chromosomal anomalies. All studies were performed for clinical diagnostic purposes. Gestational ages of our cases ranged from 19 to 34 weeks of gestation (mean: 25). The cromosopathy were subdivided as follows: 14 cases of trisomy (complete, partial or mosaicism), 4 cases of deletions, 3 cases of traslocations, 5 cases of sexual chromosomes anomalies. MR imaging studies were performed on a 1.5 Tesla unit with surface or body coils. Multiplanar T2-weighted single- shot-fast spin-echo (ss-FSE) sections were acquired. Clinical or radiological follow-up with post-natal ultrasound or MR imaging was obtained in 11 cases, confirming prenatal findings. Abortion was performed in 6 cases; two underwent MR autopsy. In 9 cases we had not follow-up.

RESULTS

Among our 26 cases only two showed normal brain on MR imaging. The others 24 fetuses presented brain anomalies that ranged from subtle findings to very important malformed pictures. Nine cases referred to well known genetic syndrome (trisomy 13: three cases, trisomy 21: two cases, trisomy 18: one case, Turner syndrome: one case, Klinefelter syndrome: two cases). Seventeen fetuses referred to rarer chromosomal anomalies. Their MR imaging findings include cerebellar hypoplasia, ventricular dilatation, persistence of falcine sinus, cortical malformations.

\section{CONCLUSIONS}

As genetic evaluation is becoming more and more common and precise we tried to understand if and when fetal brain dysmorphic features may suggest or support a chromosopathy underlying the value of also subtle morphological anomalies of brain structures. Combination of chromosomal analysis and fetal MR imaging data will help to establish a more correct diagnosis and prognosis of these anomalies. 


\section{LP01:16:7}

\section{FOREBRAIN DEVELOPMENT IIN FETAL MRI:} DETECTABILITY BEFORE GESTATION WEEK 27

\author{
M. Schmook, P. Brugger, M. Weber, G. Kasprian, \\ D. Prayer \\ Medical University of Vienna, Vienna, Austria
}

\section{PURPOSE}

Forebrain malformations are one of the most severe developmental anomalies. They are often associated with face dysmorphism and require early diagnosis. Intracranial landmarks of normal forebrain development include cleavage of hemispheres, presence of olfactory structures, the pituitary gland and the corpus callosum. While for example olfactory structures are known to be well depicted in in vivo MRI from gestational week (gw) 30 onwards, data about their morphological proof in earlier gw is not yet available.

The purpose of this retrospective study was to evaluate the detectability of anatomical landmarks of forebrain development in in-vivo fetal MRI before gw 27.

PATIENTS AND METHODS

MRI studies of 52 fetuses (gw 16-26, average \pm std: $22 \pm 2.4$ ) performed with a 1.5 Tesla magnet were assessed. T2 weighted (w) fast spin-echo sequences in 3 orthogonal section planes, T1w gradient-echo and diffusion-weighted sequences were screened for the detectability of olfactory bulbs, tracts and sulci, the interhemispheric division, infundibulum, pituitary gland, corpus callosum, chiasma, palate and profile. Statistical analysis was performed using SPSS (11.5.1 SPSS Inc., Chicago, Ill).

\section{RESULTS}

The interhemispheric division, corpus callosum, chiasma, profile and palate were detectable in $96 / 92 / 88 / 82$ and $76 \%$ of cases.

Olfactory tracts could be depicted in $39 \%$ of cases, with a significant better delineation after gw $21(\mathrm{p}=0.034)$. They were easier to depict than the olfactory bulbs and sulci (14-18\%). Coronal $\mathrm{T} 2 \mathrm{w}$ sequences were the most sensitive.

The pituitary gland could be detected in $81 \%$ of cases, with a significant increase of delineation after gw 20. $(\mathrm{p}<0.05)$. Its maximum diameter was 3 to $6 \mathrm{~mm}$, increasing with gw.

Pathologies were found in $21 \%$ of cases.

\section{CONCLUSION}

For evaluation of forebrain development, the interhemispheric division, corpus callosum, chiasma, profile and palate are readily detectable in early fetal MRI. Identification of olfactory structures requires a coronal T2w sequence paralleling the course of the brainstem, and is possible in about one third of cases between gw 16 and 26.

Figures:

a.) Coronal $\mathrm{T} 1 \mathrm{w}$ MR image showing hyperintense pituitary gland (gw 21)

b.) Coronal diffusio weighted MR image demonstrating the corpus callosum (gw 22)

c.) Coronal T2w MR image with the olfactory tracts (gw 24)

d.) Sagittal T2w MR image demonstrating the fetal profile (gw 23)
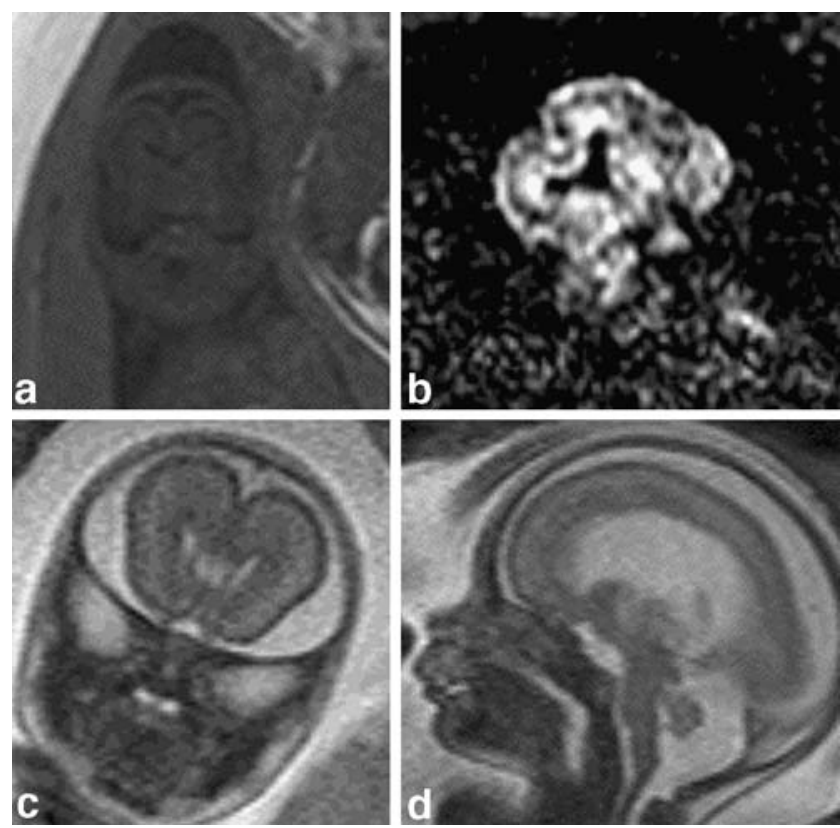

\section{LP01:16:8}

\section{DEEP GREY MATTER AND BRAINSTEM \\ INVOLVEMENT IN THE TERM NEONATE \\ WITH HYPOXIC ISCHAEMIC ENCEPHALOPATHY \\ SEEN ON BRAIN MRI}

$\underline{\text { R. Gunny }}^{1}$, C. Hagmann ${ }^{2}$, A. Kapetanakis ${ }^{2}$, W. Chong ${ }^{1}$, N. Robertson $^{2}$

${ }^{1}$ Radiology, Great Ormond Street Hospital and Institute of Child Health, London, United Kingdom; ${ }^{2}$ Institute of Women's Health, Centre for Perinatal Brain Research, University College London, London, United Kingdom

\section{INTRODUCTION}

Two distinct MRI patterns of brain injury are seen in HIE in the term neonate: one of cortical-subcortical 'watershed' infarction, the other affecting the ventrolateral thalamus/posterolateral putamen. Brainstem injury is uncommon, attributed to early death and lack of MRI data in this group. Histopathological studies indicate the basal ganglia/thalamic pattern includes other deep grey matter structures such as the subthalamic nucleus (STN), amygdalahippocampal formation (AHF), lateral geniculate bodies (LGB) and brainstem.

AIM

To delineate the extent of deep grey matter and brainstem involvement in HIE in the term neonate detected on clinical brain MRI scans obtained at $1.5 \mathrm{~T}$.

\section{METHODS}

The brain MRI scans of 20 consecutive term babies admitted to the neonatal unit at University College Hospital (London) with HIE grades 1-3 were reviewed by 2 paediatric neuroradiologists. T2-weighted fast spin echo (TR/TEeff $=5.91 / 110,3 \mathrm{~mm}$ slices), T1-weighted 3D-FLASH $\left(\mathrm{TR} / \mathrm{TE}=6.06 / 17\right.$, flip angle $21^{\circ}, 1 \mathrm{~mm}$ slices), DWI (double spin echo EPI TR/TE $=4200 / 118,3 \mathrm{~mm}$ 
slices) and calculated ADC maps were acquired on a Siemens Avanto $1.5 \mathrm{~T}$ scanner. Evidence of regional involvement was defined as the presence of signal or diffusion abnormality, enlargement or atrophy compared to normal term controls.

RESULTS

All 20 babies were scanned within 2 weeks of the acute clinical presentation, (range 12 hours-14 days). Regional distribution of MRI abnormality is shown in Table 1. AHF, STN and LGB abnormalities were not seen without basal ganglia/thalamic injury. 4/12 with basal ganglia abnormality had brainstem abnormality outside the corticospinal tracts; 4/8 with AHF abnormality had brainstem abnormality.

\begin{tabular}{|c|c|c|c|c|c|c|c|c|c|c|}
\hline $\begin{array}{l}\text { Table } 1 \\
\text { Regional } \\
\text { distribution }\end{array}$ & & & & & ocati & & & & & \\
\hline & Putamen & \begin{tabular}{|l|l|l} 
Caudate \\
\end{tabular} & \begin{tabular}{|l} 
Globus \\
Pallidus
\end{tabular} & Thalamus & STN & \begin{tabular}{|l|l} 
AHF \\
\end{tabular} & LGB & Midbrain & Pons & Medulla \\
\hline $\begin{array}{l}\text { MRI } \\
\text { Abnormality } \\
(/ 20)\end{array}$ & 10 & 9 & 10 & 12 & 6 & 8 & 5 & 4 & 4 & 3 \\
\hline
\end{tabular}

\section{CONCLUSION}

Deep grey matter involvement seen on MRI in severe HIE includes grey matter structures caudal to the basal ganglia/thalami. There is evidence of a rostro-caudal pattern of involvement with increasing severity of injury.

\section{REFERENCES}

${ }^{1}$ Barkovich AJ. MR and CT evaluation of profound neonatal and infantile asphyxia. AJNR 13(3): 959-72

${ }^{2}$ Schneider H. Anoxic encephalopathy with predominant involvement of basal ganglia, brain stem and spinal cord in the perinatal period. Report on seven newborns. Acta Neuropathol (Berl) 32(4): 287-98 1975

${ }^{3}$ Dambska M, Myers RE. Topography of lesions in newborn and infant brains following cardiac arrest and resuscitation. Damage to brain and hemispheres. Biol Neonate. 29(3-4): 194-206 1976

\section{LP02}

09.55-10.55 - Advanced imaging modalities: anatomy, angio, and new applications

\section{LP02:17:1}

\section{IN VIVO 3T MR SPECTROSCOPY OF CERVICAL SPINAL CORD}

A.Marliani ${ }^{1}$, V. Clementi ${ }^{2}$, R. Agati ${ }^{1}$, L. Albini Riccioli ${ }^{1}, \mathrm{M}$. $\overline{\text { Leonardi }^{1}}$

${ }^{1}$ Neurosciences Dtp. Bellaria Hospital, Bologna, Italy;

${ }^{2}$ GE Healthcare Technologies, GE Healthcare Italy, Bologna, Italy

\section{INTRODUCTION}

MR spectroscopy has been widely used to study cerebral diseases, however a lot of CNS pathologies involve also the spinal cord.

Because of the technical difficulty to perform spectroscopy on spinal cord, due to magnetic field inhomogeneities physiological movement and size of the anatomical region of interest, these studies are limited and not still realized with 3T system We report the preliminary results on healthy volunteers.

SUBJECTS AND METHODS

We performed spinal cord MRS exams on 10 healthy volunteers, whit a 3T whole-body Signa Excite system using a standard 8 channels phased array CTL coil. Spectra were obtained by a PRESS sequence with TE $35 \mathrm{~ms}$, TR $2000 \mathrm{~ms}$, 400 averages and CHESS to provide water suppression. Arectangular VOI along the main axis of the cord with dimensions of approximately $7 \times 9 \times$ $35 \mathrm{~mm}$, was localized between the C2-C3 level. For each metabolites spectrum sixteen additional acquisitions with unsuppressed water were collected. Automated optimisation of gradient shimming, transmitter pulse power, and water suppression were used.

Data post processing was performed using the LC model and SAGE (GE, Milwaukee).

\section{RESULTS}

The spectra obtained with an acquisition time of 14 minutes showed a good quality (with a unsuppressed water line-width of approximately $12 \mathrm{~Hz}$ for all subjects) and was adequate to evaluate NAA, Cr, Cho signals.

Conclusion: These preliminary results demonstrated that a spinal cord $1 \mathrm{H}-\mathrm{MRS}$ study with a $3 \mathrm{~T}$ system is possible and reliable. This technique can be potentially useful also on spinal cord diseases.

\section{LP02:17:2}

\section{VENOUS ANATOMY OF THE PARASELLAR REGION AND THE SKULL BASE : VALUE OF ANGIOGRAPHIC COMPUTED TOMOGRAPHY (ACT, DYNACT) AND DUAL VOLUME TECHNIQUE (DVT)"}

G. Benndorf, R. Klucznik, D. Meyer

The Methodist Hospital, Weill Cornell University, Houston, New York, USA

\section{BACKGROUND AND PURPOSE}

Venous anatomy of the parasellar skull base is complex and remains difficult to understand even when imaged using modern 3D-MRI or 3D-CTA. We report on our experience in studying a new software mode Dual-Volume-technique (DVT) for 3D-DSA, combined with contrast enhanced ACT (DynaCT) and its value for visualizing venous structures at the skull base.

\section{MATERIAL AND METHOD}

In four patients with arteriovenous shunting lesions of the CS and in four patients undergoing petrosal sinus sampling, an arterial and / or venous 3-DSA was performed

using a $\mathrm{C}$-arm mounted flat detector system (AXIOM Artis dBA, Siemens medical Solution) and the following parameters: $10 \mathrm{sec}$, $2.5 \mathrm{cc} / \mathrm{sec}$, total of $28 \mathrm{ml}$ (300 mg Iodine) Dual volumes were 
obtained by separate reconstructions of mask and filling run and subsequent fusion using a dedicate commercially available workstation (Leonardo Siemens). Additional ACTs (DynaCTs) were performed the following parameter: 20sec. rotations, 0.4 increment, $220^{\circ}$ total angle, 1024 matrix, 538 projections."bone, sharp", $512 \times 512$ ' reconstruction mode. Image post-processing was performed using maximum intensity projections (MIPs). Contrast enhancement was achieved using $20 \%$ or $50 \%$ dilution (300 mg Iodine), $2 \mathrm{cc} / \mathrm{sec}$ and total of $40 \mathrm{ml}$.

\section{RESULTS}

Visualization of major and minor venous structures using both, DVT and ACT was excellent and superior to 2D-DSA. ACT (Dyna-CT) visualized reliably small and complex venous structures such as the anterior condylar confluens, the internal carotid artery plexus (Rektorzik) and the inferior petroclival vein (IPCV), often times obscured on standard 2D-DSA. DVT provided in addition information on the precise relationship between venous channels and their adjacent osseous structures or surrounding canals, not obtainable with other currently available imaging techniques.

\section{CONCLUSION}

DVT in combination with DynaCT is capable of providing a new level of anatomical vascular information that is of high value for improving knowledge and understanding of regions with complex vascular anatomy such as the parasellar region and the skull base. This knowledge can become crucial for interventional neuroradiologists when performing endovascular treatment and neurosurgeons when performing open surgery in this area. The quality of image information obtained is comparable to colored plastic casts obtained from cadavers, currently the reference method when studying vascular anatomy in this area.

\section{LP02:17:3}

WHOLE BODY CT TRAUMA IMAGING WITH ADAPTED CT- ANGIOGRAPHY OF THE CRANIO-CERVICAL VESSELS: INCIDENCE OF ASSOCIATED VESSEL INJURY

\author{
S. Langner $^{1}$, S. Fleck ${ }^{2}$, M. Kirsch ${ }^{1}$, J. Baldauf ${ }^{2}$, \\ N. Hosten \\ ${ }^{1}$ Institute for Diagnostic Radiology and Neuroradiology, Ernst- \\ Moritz-Arndt University, Greifswald, Germany; \\ 2 Department of Neurosurgery, Ernst-Moritz-Arndt University, \\ Greifswald, Germany
}

\section{OBJECTIVE}

The incidence of traumatic craniocervical artery dissection varies in published trauma series and is associated with cervical spine trauma. The purpose of this study was to determine the frequency of traumatic craniocervical artery injury in polytrauma patients by using a standardized whole body trauma CT with an adapted CT angiography (CTA) of the craniocervical vessels. METHODS

273 patients requiring a whole body trauma $\mathrm{CT}$ due to the mechanism of their injury were analyzed retrospectively. All examinations were performed using a 16 row MSCT (Sensation 16, Siemens, Erlangen) in the last 12 months.
Initially a spiral scan of the neurocranium was acquired.

Then a CT angiography of the craniocervical vessels with $40 \mathrm{ml}$ of iodinated contrast agent (flow $4 \mathrm{ml} / \mathrm{s} ; 40 \mathrm{ml}$ saline flush, flow $4 \mathrm{ml} / \mathrm{s}$ ) was performed starting at the level of Th2 up to the roof of the lateral ventricles $(100 \mathrm{kV}, 120 \mathrm{mAs}$, collimation $16 \times 0.75)$. The scan was started using bolus tracking.

Finally a contrast-enhanced spiral thoraco-abdominal scan was performed with a delay of $20 \mathrm{sec}$ after administering a second contrast bolus of $60 \mathrm{ml}$.

The craniocervical vessels were analyzed in the source images and using $20 \mathrm{~mm}$ thin slice MIP-reconstructions.

RESULTS

CTA was diagnostic in all patients.

Isolated cervical spine injuries were observed in 11 patients (4\%), isolated cranial injuries in 59 patients $(21,7 \%)$ whereas combined cervical and cranial lesions were seen in 5 patients $(1,8 \%)$.

Injuries of craniocervical vessels were observed in 2 cases $(0,98 \%)$ in isolated cervical and skull base fracture respectively and in one case without any osseous trauma.

\section{CONCLUSION}

Craniocervical vessel injury is a rare event in case of high velocity injury. Whole body trauma CT scan with an adapted scanning protocol for the craniocervical vessels is a fast, safe and feasible method for detecting vascular injuries in the craniocervical region. It allows prompt further treatment if necessary

\section{LP02:17:4}

\section{IDENTIFICATION OF THE BRAINS VESSELS BY MEANS OF MRI AND STEREOTACTIC DSA FUSION}

C. Macedo de Freitas ${ }^{1,2}$, M. Braun ${ }^{1}$, G. Cabral de Andrade ${ }^{3}$, N. Pinheiro ${ }^{4}$, A. Lebedinski ${ }^{1}$, R. Anxionnat ${ }^{1}$, D. Iancu Gontard ${ }^{1}$, L. Picard ${ }^{1}$, S. Bracard ${ }^{1}$

${ }^{1}$ Neuroradiology department, Nancy, France; ${ }^{2}$ Departamento de neurologia e psiquitria, Botucatu, Brazil; ${ }^{3}$ Centro intergrado de neurologia e neurocirurgia, Maringa, Brazil;

${ }^{4}$ Hospital Madre Teresa, Belo Horizonte, Brazil

\section{PURPOSE}

To developed a robust method of identifying arterial and venous brain vessels on injected MRI.

\section{METHODS}

In this retrospective study, we analysed injected MRI and stereotactic DSA in 23 patients submitted to radiosurgery for treatment of arteriovenous malformations. We used the Brain Lab software (GE), which allows high resolution axial T1 weighted injected MRI, merged with stereotactic DSA. The medium and small size vessels were identified in MRI by selecting 193 points which were simultaneously visualized on DSA images. Double lecture was performed by two senior radiologists. The venous points were located in the posterior fossa (34 points), in deep venous system (62 points) and in superficial veins ( 15 points). The arterial points were located in intracranial carotid artery (25), anterior cerebral artery (15), middle cerebral artery (12), posterior 
communicating artery (12), basilar artery (8) and posterior cerebral artery (19).

\section{RESULTS}

We obtained a precise correlation in 174 points and a slight gap less than $1 \mathrm{~mm}$ in 21 points. In the latter, the points were not exactly centred in the vessel lumen but always reaching the lateral wall of the vessel. No false positive points were noted. MRI then DSA separate identification were performed. The course of these vessels was then easily followed on MRI allowing precise exploration of their variability. These different patterns were identical to those provided by the DSA exploration.

\section{CONCLUSION}

Accurate identification of medium and small size vessels on high resolution injected MRI scan is reliable and may be of clinical interest to appreciate the vascular relationships of tumors and functional areas of the cortex. This should increase the standard MR examinations potentials in predicting patients' post-surgical outcome when a venous or arterial infarction occurs.

\section{LP02:17:5}

\section{EVALUATION OF 3D DYNAMIC MR ANGIOGRAPHY SEQUENCE «TRICKS »IN THE DIAGNOSIS AND FOLLOW-UP OF CEREBRAL VENOUS THROMBOSIS}

\author{
M. Tisserand $^{1}$, S. Rodrigo ${ }^{2}$, S. Foscolo ${ }^{1}$, O. Naggara ${ }^{2}$, \\ E. Schmitt $^{1}$, R. Anxionnat ${ }^{1}$, J. Meder ${ }^{2}$, S. Bracard ${ }^{1}$ \\ 1 Department of Diagnostic and Therapeutic Neuroradiology, \\ University Hospital, Nancy, France; ${ }^{2}$ Department of Neuroradi- \\ ology, Sainte-Anne Hospital, Paris, France
}

\section{PURPOSE}

To evaluate dynamic 3D MR angiography "TRICKS" (GE Healthcare) in the diagnosis and follow-up of cerebral venous thrombosis (CVT).

MATERIAL AND METHOD

TRICKS sequence was performed in 32 patients with clinically suspected CVT and in the follow-up of 16 other patients. A set of classical MRI sequences (T1 SE, T2 SE, Diffusion, 3D T1 GE with gadolinium) and TRICKS sequence were independently reviewed and compared.

\section{RESULTS}

There was a perfect agreement between classical MRI sequences and TRICKS sequence in the diagnosis of CVT. Both agreed in the positive diagnosis (10 patients) and in the location of the thrombus including cortical veins. In the follow-up of $16 \mathrm{CVT}$, recanalization was complete in 9 and partial or absent in 7 and there was also a complete agreement between classical sequences and TRICKS sequence.

\section{CONCLUSION}

TRICKS sequence provides reliable images for the diagnosis and follow-up of CVT in perfect agreement with classical sequences and gives in addition hemodynamic information. Further evaluation is needed but TRICKS could become the reference sequence for CVT.

\section{LP02:17:6}

DEMONSTRATION OF THE CENTRAL CEREBRAL VEINS BY SUSCEPTIBILITY- WEIGHTED IMAGING (SWI)

\author{
$\underline{\text { C. Plank }}^{1}$, C. Mueller-Mang ${ }^{1}$, G. Kasprian ${ }^{1}$, \\ $\overline{\text { P. Brugger }}^{2}$, D. Prayer ${ }^{1}$ \\ 1 Department of Radiology, Medical University of Vienna, \\ Vienna, Austria; ${ }^{2}$ Center of Anatomy and cell Biology, Vienna, \\ Austria
}

\section{PURPOSE}

to investigate the ability of SWI to demonstrate the normal course of the basal central vein, internal cerebral vein, and variations thereof.

\section{PATIENTS AND METHODS}

In 50 consecutive patients (8 to 60 years, mean 48,5) who underwent cerebral MRI for different indications, whose findings suggested absence of any venous pathology were included. All Patients were examined on a $3 \mathrm{~T}$ Scanner and additionally to the standard sequences an additional SWI sequence (TR 28, TE 20, FA 15) was performed. Images were evaluated concerning the main feeders of the basal central vein and the internal cerebral vein as well as variations of the feeders.

RESULTS

Deep cerebral veins and their feeders could be identified in each case (100\%). Evaluation included the proof of the internal cerebral vein, its feeding veins (V septi pellucidi ant, and terminalis), to its confluence to the vena Galeni, of the main orificing veins (V septi pellucidi and thalamostriata sup), basal vein and ist main feeder (anterior cerebral vein) to its orifice into the vena Galeni or Sinus rectus. Variations of the feeders of the internal cerebral vein were depicted in 10 cases, in 5 cases small subtle deep venous malformations were found. CONCLUSION

The main deep vessels responsible for the drainage of the brain and their variations are readily and reliably identifiable on SWI. Variations as well as subtle malformations of deep cerebral veins are depicted accurately. Thus SWI holds the potential to become an important non-invasive modality for investigation of venous malformations.

\section{LP02:17:7}

\section{THE PINEAL GLAND REVISITED WITH HI-RESOLUTION TRUE-FISP-IMAGING - IMPLICATIONS FOR THE DIAGNOSIS OF PINEAL ABNORMALITIES}

I. Nolte, J. Scharf

$\overline{\text { Faculty }}$ of clinical Medicine Mannheim of the University of Heidelberg, Mannheim, Germany 


\section{PURPOSE}

Pineal abnormalities are occasionally detected with routine MRI sequences(prevalence of pineal cysts: $2-10 \%$ ). The prevalence of pineal abnormalities detected with autopsy is much higher (3040\%). 3D-FISP-imaging is often used for the high-resolution imaging of the inner ear or the cisternal course of the cranial nerves. It is therefore promising to use the 3D-FISP-imaging for the exact delineation of small structures like the pineal gland.

The purpose of this study was to compare the prevalence and the morphology of pineal cysts with routine MRI sequences to highresolution 3-D-FISP-imaging.

METHODS

To compare the diagnostic yield with high-resolution 3- D-FISPimaging with that of standard sequences (T1, T2, FLAIR, dwi, $5 \mathrm{~mm} \mathrm{sl}$ th) 110 patients with no history of pineal gland changes received standard sequences and additional high resolution 3-D FISP imaging. The images were evaluated by two experienced neuroradiologists for the presence of pineal cysts, size of pineal cysts and configuration of the cysts as well diffusions restriction of the cysts

\section{RESULTS}

The prevalence of pineal cysts was $37 \%$ with the FISP-sequence compared to $12 \%$ in T1-wi, $6 \%$ in T2-wi and $10 \%$ in FLAIR-wi. In contrast to previous studies, most of the cysts did not show diffusion restriction. The FISP sequence was superior in detecting abnormal cyst configuration as well as in exactly defining of the size of the cyst.

\section{CONCLUSION}

We conclude that additional high-resolution 3-D-imaging is mandatory for the exact diagnosis of pineal abnormalities and should be used for the diagnosis and follow-up of pineal abnormalities.

\section{LP02:17:8}

\section{ECG TRIGGERED CINE-TRUEFISP}

FOR DEMONSTRATION OF VENTRICULAR AND CSF MOVEMENTS

\section{B. Schmitz, A. Aschoff, S. Klein}

Clinic for diagnostic and interventional Radiology, University Hospitals Ulm, Ulm, Germany

\section{PURPOSE}

The pulsatile blood flow causes dynamic changes within the bony skull which mainly is compensated by the ventricles and CSF flow. Cine-TrueFISP is a dynamic ECG synchronized MRsequence which is mainly used for visualizing dynamic processes of the heart. However its capability to show ECG related changes in a movie like fashion make it ideal to study ECG related dynamics in other body regions as well.

The purpose of the present study is to demonstrate the possibilities of this MR-technique for neuroradiological applications.

\section{METHODS}

At first the method has been tested in normal volunteers to demonstrate regular ventricular movements. After establishing the technique it has been used in patients with cranial and spinal arachnoid cysts, Foramen Monroi related tumors or cysts and ventriculostomy controls.

Typical sequence parameter were: Siemens Symphony $1.5 \mathrm{~T}$, ECG-gated Cine-TrueFISP TR 22.88, TE 5.72, bandwidth $130 \mathrm{~Hz} / \mathrm{Px}, 512 \times 512$ matrix, single slice $3 \mathrm{~mm}$ thickness, temporal resolution $25 \mathrm{~ms}$ depending on the cardiac cycle.

RESULTS

ECG-triggered CineTrueFISP turned out to be a method with high CSF contrast as well as high temporal and spatial resolution. In contrast to other CSF optimized sequences like CISS it resolves moving parts of the ventricles like the terminal lamina and floor of the third ventricle with great detail. The only difficulties were susceptibility related ring like artifacts and the need to view the images as movies on electronic systems.

In patients with arachnoid cysts the sequence demonstrates the wall of the cyst and its movement (see figure with cyst wall moving synchronous to ECG) and depicts compression effects with great detail.

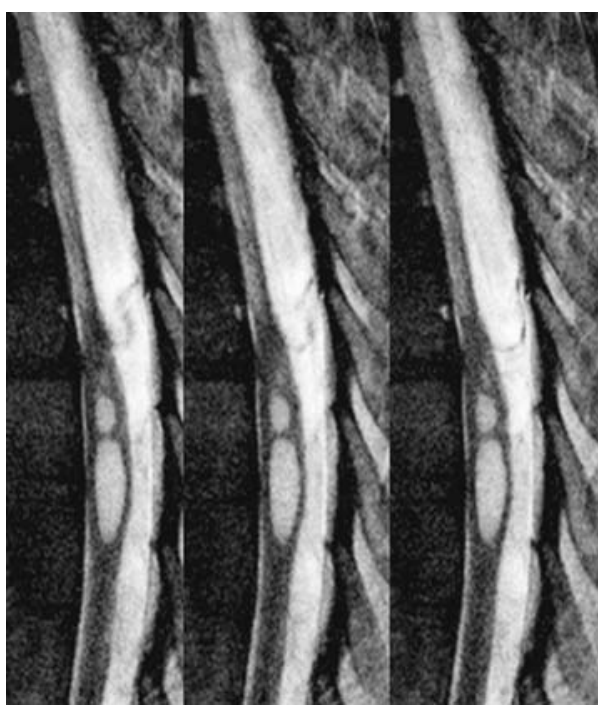

\section{CONCLUSION}

The use of ECG triggered cine-TrueFISP for imaging CSF-flow related pathologies like arachnoidal cysts, ventricular movements or control of ventriculostomy is highly recommended. It is well suited as an adjunct to phase-contrast CSF-flow quantifications.

\section{LP02:17:9}

\section{DEPENDENCIES OF MRI CONTRAST MEDIA PROPERTIES: MAGNETIC FIELD-STRENGTH, SOLVENT AND PULSE SEQUENCE}

M. Rohrer, H. Bauer, H. Weinmann

Bayer Schering Pharma AG, Berlin, Germany 


\section{PURPOSE}

To quantitatively compare magnetic properties of $\mathrm{Gd}$ containing MRI contrast media at different field strengths, based on: 1.) calculated T1-shortening based on standardized relaxivity measurements and literature data. 2.) actual signal intensity measurements using standard imaging sequences at various field strengths. METHODS AND MATERIALS

Commercially available formulations of MRI contrast media Magnevist ${ }^{\circledR}$, Gadovist ${ }^{\circledR}$, Vasovist ${ }^{\circledR}$ [Bayer Schering Pharma AG, Berlin] and MultiHance ${ }^{\circledR}$ [Bracco, Milan]) were diluted to 'physiological' concentrations with plasma. Imaging sequences were applied at $37^{\circ} \mathrm{C}$ with standard $\mathrm{T} 1$-weighted techniques such as SE (400/10) and fast GRE at field strengths ranging from 1.5 to $9.4 \mathrm{~T}$.

\section{RESULTS}

All solutions displayed qualitatively similar curves: the concentration-dependent signal intensity function has a maximum, shifting to lower concentration at higher magnetic field strength. Protein binding agents (MultiHance ${ }^{\circledR}$, Vasovist ${ }^{\circledR}$ ) show a more pronounced field strength dependence, based on their biophysical mode of action.

\section{CONCLUSION}

Optimal signal intensity (enhancement) of contrast media occurs at different concentrations and is not only related to their $\mathrm{T} 1$ relaxivity. Correlation of the achievable signal enhancement, as measured with standard imaging sequences or calculated from relaxivity data, is generally good but depends both on concentration ranges and protein-binding properties. In order to fully exploit the diagnostic potentials of the different MRI contrast media for CNS MRI, their specific magnetic properties and field strength dependencies should be carefully taken under consideration.

\section{LP03}

10.55-11.50 - Advanced imaging modalities: diffusion

\section{LP03:18:1}

\section{ON THE CONSTRUCTION OF A HEALTHY BRAIN INTER- SUBJECT DIFFUSION TENSOR IMAGE AND TRACTOGRAPHY ATLAS}

\author{
W. Van Hecke ${ }^{1}$, E. D'agostino ${ }^{2}$, A. Leemans ${ }^{3}$, \\ P. Parizel ${ }^{4}$, F. Maes ${ }^{2}$, J. Sijbers ${ }^{1}$ \\ 1 VisionLab, Department of Physics, University of Antwerp, \\ Antwerp, Belgium; ${ }^{2}$ Medical Image Computing,Department of \\ Electrical Engineering, Leuven, Belgium; ${ }^{3}$ CUBRIC, Department \\ of Psychology, Cardiff, United Kingdom; \\ 4 Department of Radiology, University of Antwerp, Antwerp, \\ Belgium
}

\section{INTRODUCTION}

Diffusion tensor imaging (DTI) has become increasingly important in neuroscience research, since it is the only technique that can investigate the structure and properties of white matter (WM) in-vivo and non-invasively. The WM fiber bundle pathways can be reconstructed using diffusion tensor tractography (DTT). The goal of this work is to create a DTI atlas that includes all directional diffusion information with a minimal bias towards any specific individual template. This subject-independent DTI atlas, containing all available diffusion information, can then be used as a template in future voxel-based morphometry(VBM) studies or to construct atlases of different WM pathologies.

\section{METHODS}

DTI of the brain was performed with a $1.5 \mathrm{~T}$ scanner on 20 healthy subjects. For the atlas construction, all images were registered to each other by the use of a viscous fluid model and mutual information[1]. The registration was based on all DT elements to provide an optimal orientational alignment. The atlas methodology is depicted in the Figure(A). The deformation fields of an image to all other images are averaged and applied to the image. This is done for all images and the resulting deformed images are averaged.

\section{RESULTS}

The proposed method was compared with the subject-dependent atlas methodology of Guimond et al.[2]. The results demonstrate that our method is able to optimally capture the directional diffusion information and is therefore more robust during the subsequent DTT. Different WM pathways can be visualized (see Figure(B,C,D)).

\section{CONCLUSION}

A subject-independent DTI atlas that contains all directional diffusion information is constructed. The higher signal-to-noise ratio, and the averaging of 'spurious' fibers allows a robust visualization of the WM tracts of a healthy population. In future work, this atlas will be used as a basis for VBM studies and to visualize WM connectivity alterations in different pathologies.

[1]VanHecke,etal.,Multi-channel Coregistration of DTI based on a Viscous Fluid Model,IEEE/EMBSBeneluxSymposium,139-142, (2006)

[2]Guimond,etal.,Average brain models:a convergencestudy,ComputVisionImagUnderstand,77,192-210(2000)
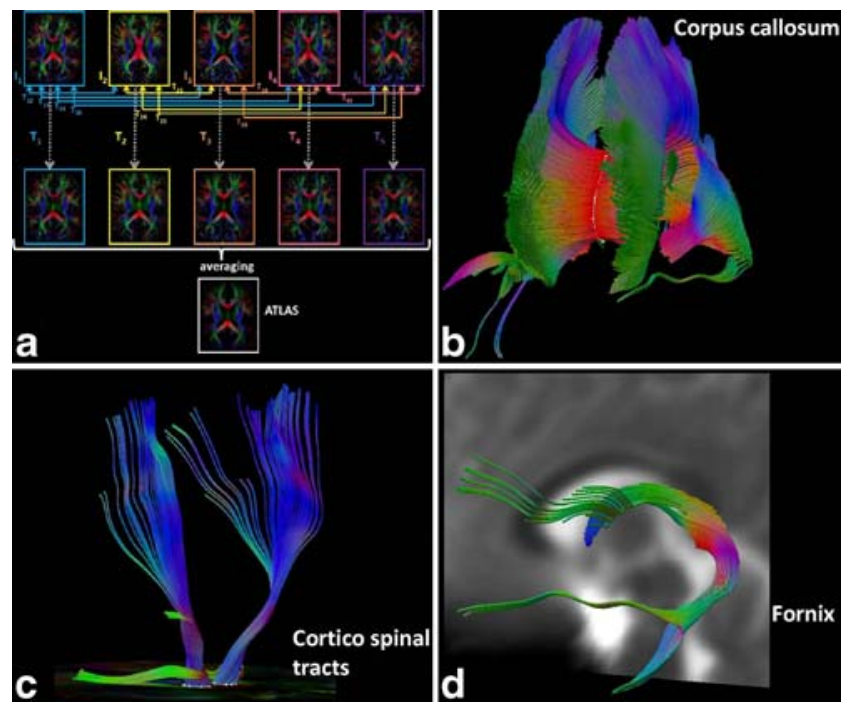


\section{LP03:18:2}

COMPARISON OF CEREBRAL BLOOD FLOW, CEREBRAL BLOOD VOLUME, APPARENT DIFFUSION COEFFICIENT AND FRACTION ANISOTROPIC VALUES IN NORMAL HEALTHY CONTROLS EXAMINED ON BOTH A 1.5

P. Sundgren, G. Beyer, J. Williamson, F. Londy,

S. Rohrer, T. Chenevert

Department of Radiology, University of Michigan, Ann Arbor, USA

\section{PURPOSE}

To evaluate for possible significant differences in cerebral blood flow (CBF), cerebral blood volume (CBV), apparent diffusion coefficient (ADC) and fraction anisotropy (FA) in healthy subjects examined on both $1.5 \mathrm{~T}$ and $3 \mathrm{~T}$ MR scanners.

MATERIAL AND METHODS

PWI and DTI were performed in eleven healthy controls examined on both a 1.5 T scanner (LX EchoSpeed, GE Medical Systems) and a 3 T scanner (Achieva, Philips Medical Systems). ROIs were placed in frontal white matter (FW), basal ganglia (BG), parietal white matter (PW) and occipital gray matter (OG) on generated CBV and $\mathrm{CBF}$ maps and in corpus callosum (CC), right and left internal capsule (IC), FW and PW on generated ADC and FA maps. Paired T-test was used for statistical significance ( $\mathrm{p}$-value $<0.05$ ).

RESULTS

The CBF was significantly lower in BG and OG $(23.5 \% \quad(p=$ $0.001), 24.4 \%(p=0.005))$ and CBV significant lower in all three locations $(11 \%(p=0.04), 28.8 \%(p=0.006), 18 \% \quad(p=0.007))$ on the $3 \mathrm{~T}$ compared to $1.5 \mathrm{~T}$.

No significant differences in FA values were seen. The ADC values were significant lower in FW $(p=0.007)$, in PW $(p=0.01)$ and in the right IC $(\mathrm{p}<0.01)$ on the $3 \mathrm{~T}$ compared to $1.5 \mathrm{~T}$ scanner. DISCUSSION

The results indicate that apparent $\mathrm{CBV}$ and $\mathrm{CBF}$ are lower on $3 \mathrm{~T}$ compared to $1.5 \mathrm{~T}$. This could, in part, be due to differences in acquisition technique driven by resolution, SNR, and system capabilities. However the systematic reduction perfusion indices at 3T $(\sim 18-28 \%)$ is comparable to intersubject variability. In contrast the diffusion parameters seems in general to be less affected by the field strength.

\section{LP03:18:3}

\section{DTI STUDY OF THE PRINCIPAL INTERHEMISPHERIC CONNECTION IN HEALTHY INDIVIDUALS AND GENDER DIFFERENCES}

\author{
F. Pizzini $^{1}$, G. Tassinari ${ }^{2}$, A. Beltramello ${ }^{1}$ \\ ${ }^{1}$ Dpt. Of Neuroradiology, University H., Verona, Italy; \\ ${ }^{2}$ Dpt. Neurol. Sci. \& Vision, Verona, Italy
}

The aim of this study is to establish a topographical distribution of the fiber connections to the cortex in the mid-sagittal crosssections of the corpus callosum of healthy individuals.

The corpus callosum (CC) is the main commissural fiber tract connecting the right and left cerebral hemisphere. Conventional MRI of the human CC does not reveal morphologically discernable portions. Using tractography, a novel computational technique in which fiber bundles are reconstructed three-dimensionally according to DTI data, we obtain a "virtual dissection" of the different callosal portions. We applied DTI and tractography to gain cortical connectivity information of the $\mathrm{CC}$ in 40 normal subjects (20 female and 20 male). With DTI-based tractography we distinguished five portions of $\mathrm{CC}$, containing fibers projecting into prefrontal, frontal motor-premotor-supplementary, parietal/ occipital, temporal areas and capsular/nuclear regions. We also analyzed the DTI microstructure information (FA, DWI, ADC) of the different callosal portions, and its gender differences. The results are similar to those described in previous DTI studies (Huang et al., 2005; Hofer \& Frahm, 2006) with smaller samples, and confirm that the fibers projecting into frontal motor-premotorsupplementary areas occupy the largest subdivision of the CC, which extends far more posteriorly as compared to Witelson's scheme, as well as the existence of fibers projecting into capsular/ nuclear regions. These fibers have not been described anatomically, but reported in neurophysiological animal studies (Ezroki et al., 1985).

There is no gender difference in the volume of the fibers, but the FA values of the frontal motor-premotor-supplementary fibers are lower in the females than males and the DWI values higher in females than males.

Differences in DTI parameters within the different portions of the corpus callosum, and gender differences, raise important questions regarding its microstructure which go beyond the information provided by morphology.

\section{LP03:18:4}

INTERHEMISPHERIC CONNECTIVITY IN PATIENTS WITH CALLOSAL RESECTION DESCRIBED AND QUANTIFIED USING DIFFUSION TENSOR IMAGING

G. Polonara ${ }^{1}$, M. Fabri ${ }^{2}$, G. Mascioli ${ }^{1}$, A. Paggi ${ }^{3}$, $\overline{\text { T. Manzoni }}^{2}$, U. Salvolini ${ }^{1}$

${ }^{1}$ Istituto di Radiologia - Università Politecnica delle Marche, Ancona, Italy; ${ }^{2}$ Dipartimento di Neuroscienze - Università Politecnica delle Marche, Ancona, Italy;

${ }^{3}$ Clinica Neurologica - Ospedali Riuniti di Ancona, Ancona, Italy 


\section{PURPOSE}

The corpus callosum (CC), the main white matter structure connecting the left and right cerebral hemispheres, provides for interhemispheric transfer of information. The present diffusion tensor imaging study aimed at characterizing the microstructure of white matter tracts and at describing their trajectories in patients with callosal resection.

\section{MATERIALS AND METHODS}

Ten healthy volunteers and seven callosotomized patients gave their informed consent to participate in the study. Patients had callosal resection of varying extent to treat severe epilepsy: four had total callosotomy, two had partial anterior resection and one had partial posterior callosotomy. The study was carried out with a GE Signa 1.5 Tesla magnet. Fractional anisotropy, apparent diffusion coefficient and volume ratio anisotropy of the principal fibre bundles were determined and compared in patients and controls.

\section{RESULTS AND CONCLUSIONS}

In the two patients with partial anterior resection and in control subjects the fibres interconnecting the parieto-occipital and temporo-occipital lobes of the two hemispheres crossed the extant posterior CC. The frontoparietal opercula of the two hemispheres were connected through subcortical structures. In the patient with partial posterior resection, fibres from the frontoparietal lobe reached the contralateral hemisphere through the anterior $\mathrm{CC}$, as in control subjects. The parieto-occipital and temporo-occipital lobes were not connected to the homologous contralateral areas. In the four patients with complete resection, white matter fibres from the parieto-occipital and temporo-occipital lobes did not cross to the contralateral hemisphere. The fronto-parietal opercula of the two hemispheres were connected through subcortical structures, as in patients with anterior resection. The fibres of the anterior commissure could be identified in three patients. Corticospinal tracts and major brainstem bundles were similar in patients and controls $(\mathrm{P}>0.05)$.

The present results confirm previous neuropsychological and functional findings about the role of the $\mathrm{CC}$ in the transfer and integration of interhemispheric information.

\section{LP03:18:5}

\section{PROBABILITY MAPS OF OPTIC RADIATION IN PATIENTS WTIH HIPPOCAMPAL SCLEROSIS}

I. Mader ${ }^{1}$, V. Glauche ${ }^{2}$, H. Mast ${ }^{1}$, M. Unfried ${ }^{3}$, K. Il'yasov ${ }^{4}$, B. Kreher ${ }^{4}$

1 Section of Neuroradiology, Neurocenter of the Freiburg Universtiy Hospital, Freiburg, Germany; ${ }^{2}$ Dept. of Neurology, Neurocenter of the Freiburg Universtiy Hospital, Freiburg, Germany; ${ }^{3}$ Dept. of Neurosurgery, Neurocenter of the Freiburg Universtiy Hospital, Freiburg, Germany; ${ }^{4}$ Medical Physics, Freiburg Universtiy Hospital, Freiburg, Germany

\section{PURPOSE}

Fiber tracking of the optic radiation is often hampered by the strong bending of the Meyer loop or by mis-tracking into the temporal pole. Another problem is the definition of regions of interest, because they have to be created manually in the individual anatomical data set of the patient. The purpose of this study was to overcome the problems of tracking by using probability maps, and to create the seed points by a parameterised method.

\section{METHODS}

Eight patients with hippocampal sclerosis previous to selective amygdalohippocampectomy were investigated by Diffusion Tensor Imaging (DTI) at a $3 \mathrm{~T}$ whole body system. DTI analysis was performed by an in-house developed DTI and Fibertools software (http://www.uniklinik-freiburg.de/mr/live/arbeitsgruppen/ diffusion/fibertools_en.html). For the depiction of the lateral geniculate corpus (CGL) and for the primary visual cortex (V1), anatomical maps from the WFU Pick Atlas were normalised onto the b0 images of each individual patient data set by using spm5. These maps were imported into the DTI and Fibertools software and used as seed points. From these seed points probability maps were calculated for each optic radiation. For further quantitative evaluation of the probabilities of the optic radiation, additional ROIs were created as orbitals around each CGL (Fig. 1). From the cross sections of the optic radiation and the orbitals, the calculated probabilities were extracted and pooled into a pathologic side and a healthy side. Additionally, the number of found pixels containing probabilities of optic radiation was also extracted for the pathologic and the healthy side, respectively. A Wilcoxon Rank test was performed.

\section{RESULTS}

In all patients, the optic radiation was equally depicted by probability maps in the orbitals nearest to the CGL and nearest to V1 on both sides (Fig. 2). Only in the intermediate part (2nd orbital) a significant difference was visible. Here, the probabilities were higher on the pathologic side, $p=0.044$. The numbers of pixels containing the optic radiation was also equal on both sides.

\section{CONCLUSIONS}

The detection of all optic radiations was better than described in the literature [ $80 \%$ by Yamamoto 2007$]$. The probability maps are similar to other published probability index maps. The voxel based approach for the selection of the seed points is an objective tool. The equal volumes of the optic radiation on the healthy and pathologic side are expected, as hippocampal sclerosis does not affect the optic radiation. It indicates that the method is a robust means for pre-operative depiction of the optic radiation. 

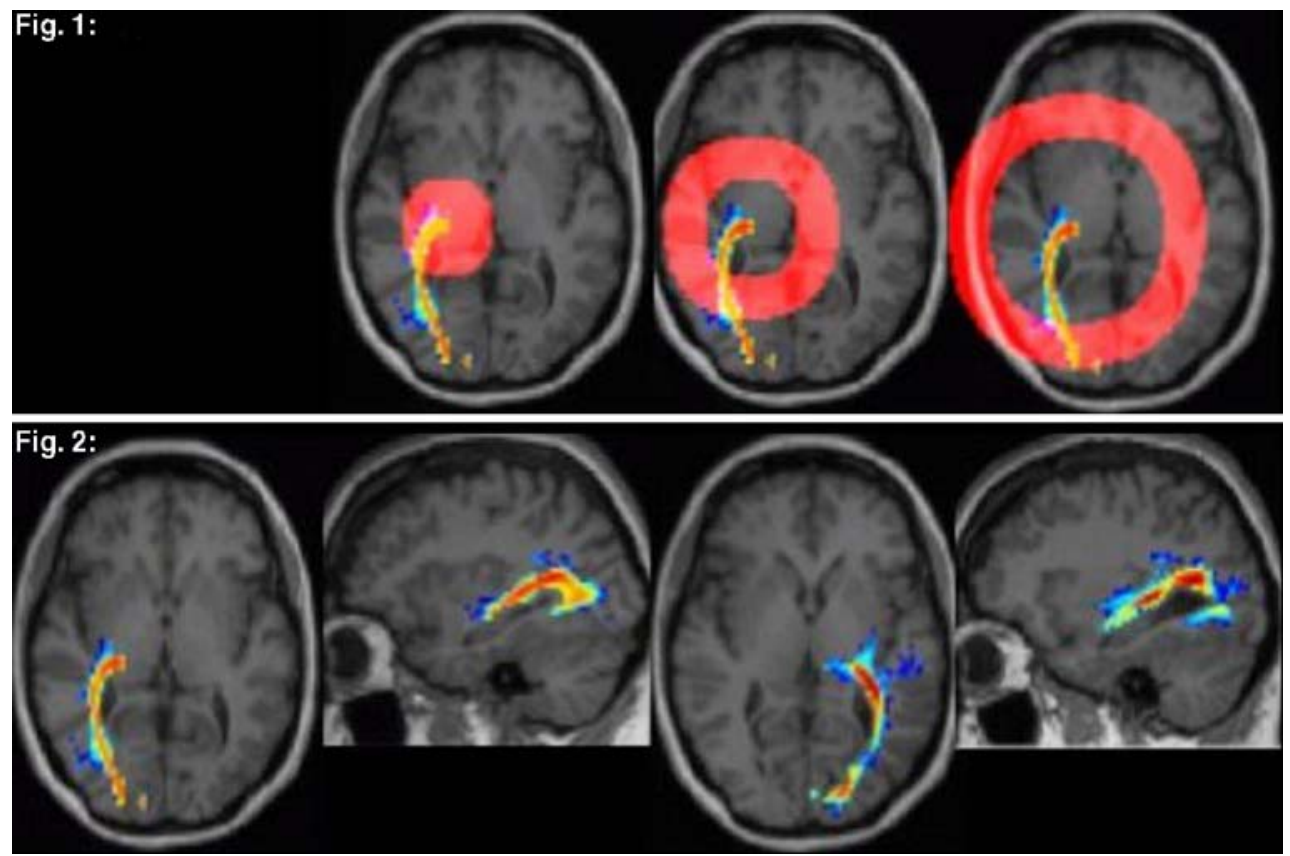

\section{LP03:18:6}

\section{ADC MEASUREMENTS AT THE GYRAL LEVEL AND OF DEEP NUCLEI IN SPORADIC CREUTZFELDT-JAKOB DISEASE}

\section{P. Vitali $^{1,4}{ }^{\text {A. R. Henry }}{ }^{2}$, S. Chung ${ }^{2}$, W. Dillon ${ }^{3}$,}

A. Haman ${ }^{1}$, B. Miller ${ }^{1}$, M. Geschwind ${ }^{1}$

${ }^{1}$ Memory and Aging Center, Neurology, UCSF, San Francisco (CA), USA; ${ }^{2}$ Center for Molecular and Functional Imaging, Radiology, UCSF, San Francisco (CA), USA; ${ }^{3}$ Neuroradiology, UCSF, San Francisco (CA), USA; ${ }^{4}$ Neuroradiology and Neurosurgery, Istituto Neurologico C.Besta, Milano, Italy

\section{INTRODUCTION}

FLAIR and/or DWI sequences are highly sensitive to cortical and subcortical grey matter abnormalities in Creutzfeldt-Jakob disease (CJD) (Young 2005; Shiga 2005) but also in rapidly progressive dementias (RPD) (Josephs 2006). Measurement of the pattern of Apparent Diffusion Coefficient (ADC) in gray matter may increase the ability to differentiate CJD from other RPDs since:

1.ADC is pure restricted diffusion (no T2 shine-through) and hence may increase the specificity for CJD in FLAIR/ DWI hyperintense areas.

2.ADC can be decreased within areas normal on FLAIR and DWI (Tschampa 2003, Lin 2006), and therefore could be more sensitive.

\section{METHODS}

Cortical ADC measurements are difficult due to CSF partial volume averaging. We have developed an automated method that minimizes the partial volume effect in ADC in 26 cortical and 5 subcortical grey matter regions (Tzourio-Mazoyer 2002) per hemisphere in order to assess regional patterns of abnormalities.

1.Multi-step non-linear registration of the Tzourio-Mazoyer templates to EPI b0 images.

2.Brain and CSF, and grey and white matter were segmented from the b0 and FA maps, respectively.

3.An ROI was defined from the overlap of the registered template and the segmented grey matter.

4.ADC values were calculated within the ROI and another approach used skeletons of the grey matter within the template regions used to get $\mathrm{ADC}$ values.

\section{RESULTS}

At the time of abstract submission, results are available on coefficient of variability for estimation of ADC in the 31 ROIs per hemisphere from 2 normal controls at 3 time points each, and 1 subject with CJD.

The coefficient of variability was typically 0.5 to 3.5 percent across the regions and subjects with an average of $2 \%$. Similar reliability was found for both skeleton and whole ROI methods. There was a trend in slightly better reliability for the whole ROI method, and lower ADC values from the skeleton method. As expected, some regions where the segmentation was more difficult due to image distortion, had worse reliability. The values for the CJD patient were much lower than the controls in most regions with the exception of the precentral and postcentral gyri.

\section{CONCLUSIONS}

We will apply these techniques to compare ADC changes in a large sporadic CJD cohort and a non-CJD RPD cohort in order to determine the patterns of ADC change that may differentiate CJD from other conditions. 


\section{LP03:18:7}

FIBER DENSITY INDEX, FRACTIONAL ANISOTROPY, ADC AND CLINICAL MOTOR FINDINGS IN WHITE MATTER OF PATIENTS WITH GLIOBLASTOMA

\section{A. Romano ${ }^{1}$, F. Fasoli ${ }^{1}$, M. Ferrante ${ }^{1}$, L. Ferrante ${ }^{2}$,} M. Bonamini ${ }^{1}$, L. Fantozzi ${ }^{1}$, A. Bozzao ${ }^{1}$

${ }^{1}$ Department of Neuroradiology S. Andrea Hospital University Sapienza, Rome, Italy; ${ }^{2}$ Department of Neurosurgery S. Andrea Hospital University Sapienza, Rome, Italy

\section{PURPOSE}

to test eventual correlation between the fiber density index (FDi), fractional anisotropy (FA), apparent diffusion coefficient (ADC) and clinical motor findings in the cortico-spinal tract of patients with glioblastomas.

\section{METHODS}

20 pts with glioblastomas (13 symptomatic for cortico-spinal tract involvement) underwent MRI, including diffusion tensor imaging (DTI) and tractography (DTT). The FDi, FA and ADC were calculated in areas of white matter in close proximity to the tumor and encompassing fibers of cortico-spinal tract and in the contralateral normal appearing white matter.

RESULTS

The mean FDi, FA and ADC values were significantly different in peritumoral white matter compared to the corresponding contralateral normal-appearing white matter. When symptomatic and asymptomatic patients were compared, the mean FDi and ADC values in the ROIs adjacent to the tumor was lower in symptomatic patients than in asymptomatic. A positive correlation was found between the clinical score (CS) and, separately, FDi, FA and ADC. In a multiple stepwise regression between the same factors, only the ADC showed a positive correlation with the CS $(0.001, \mathrm{R}=.818)$.

CONCLUSION

Increased ADC plays the main role in determining the appearance of CST symptoms in patients harboring glioblastomas.

\section{LP03:18:8}

\section{MOTOR AND LANGUAGE DTI FIBER TRACKING COMBINED WITH INTRAOPERATIVE SUBCORTICAL MAPPING IN GLIOMA SURGERY}

A. Gambini ${ }^{1}$, A. Castellano ${ }^{1}$, A. Falini ${ }^{1}$, L. Bello ${ }^{2}$, M. Fava ${ }^{2}$, M. Cadioli ${ }^{1}$, G. Scotti ${ }^{1}$

${ }^{1}$ Neuroradiology Department and CERMAC, Scientific Institute and University Vita-Salute San Raffaele, Milan, Italy; ${ }^{2}$ Neurosurgery, Department of Neurological Sciences, University of Milano, IRCCS Ospedale Maggiore Policlinico, Milan, Italy

\section{PURPOSE}

The combination of preoperative DTI-Fiber Tracking (DTI-FT) reconstruction of functional tracts with intraoperative subcortical mapping (ISM) is potentially useful to improve surgical procedures in gliomas located in eloquent areas.

Aims of the study are: 1) to evaluate the modifications of fiber trajectory induced by the tumor; 2) to validate preoperative DTIFT results with intraoperative identification of functional subcortical sites through direct subcortical stimulation; 3 ) to evaluate the impact of the availability of preoperative DTI-FT reconstructions combined to ISM technique on duration and modalities of surgical procedures, as well as on functional outcome of the patients.

\section{MATERIALS AND METHODS}

Data are available on 64 patients (52 low grade gliomas, 12 high grade gliomas). DTI-FT was acquired by a 3-T MR scanner with a singleshot EPI sequence (TR/TE $8986 / 80 \mathrm{msec}, \mathrm{b}=1000 \mathrm{~mm}^{2} / \mathrm{s}$ ) with gradients applied along 32 non collinear directions. 3D Fast Field Echo (FFE) T1-weighted imaging (TR/TE 8/4 msec) was performed for anatomic guidance. The cortico-spinal tract (CST), superior longitudinal, inferior fronto-occipital and uncinate fascicles were reconstructed, and data were transferred to the neuronavigational system. Functional subcortical sites identified during ISM were correlated with fiber tracts depicted by DTI.

\section{RESULTS}

In high grade gliomas DTI-FT depicted the tracts mostly at the tumor periphery, in low grade fibers were more frequently located inside the tumor mass, with good correlation between DTI-FT and ISM. However, for a proper reconstruction of the tracts, it was necessary to lower FA threshold of fiber tracking algorythm and to position additional ROIs, particularly in low grade gliomas and in F3 tumors. The combination of DTI-FT and ISM helped to decrease the duration of surgery, patient fatigue, and the occurrence of intraoperative seizures.

CONCLUSIONS

Combination of DTI-FT and ISM allows accurate identification of eloquent fiber tracts and enhances surgical performance and safety.

\section{LP03:18:9}

CORRELATION BETWEEN ROUTINE MRI WITH DIFFUSION WI AND HISTOLOGICAL EXAMINATION IN MENINGIOMAS: A CHALLENGE IN DIFFERENTIATING TYPICAL FROM ATYPICAL/MALIGNANT MENINGIOMAS

L. Santelli ${ }^{1}$, R. Dal Borgo ${ }^{1}$, R. Manara ${ }^{1}$, R. Mardari ${ }^{1}$, M. Severino ${ }^{1}$, R. Perini ${ }^{2}$, G. Pavesi ${ }^{2}$, E. Bavaresco ${ }^{2}$, M. Ermanni ${ }^{3}$, C. Carollo ${ }^{1}$

${ }^{1}$ Operative Unity of Neuroradiology, Padova, Italy;

${ }^{2}$ Neurosurgery Unity - Neuroscience Department, Padova, Italy; ${ }^{3}$ Neurological Clinic - Neuroscience Department, Padova, Italy

\section{OBJECT}

meningiomas can be histologically divided (according to WHO) in typical, atypical and malignant. Preoperative diagnosis may affect treatment planning and prognostication. Previous papers, on small series, have found a strict correlation between $\mathrm{ADC}$ values and histological grading, with differences even among typical subtypes. In this study we verified the presence of this correlation in a larger 
population. Moreover, classic neuroradiological signs were evaluated in different, histologically proved, meningioma subtypes.

METHODS

the study was performed on 45 patients with meningiomas (15 men, 30 women, mean age 57, age range 34-85). The MR imaging studies were performed with a 1.0 -T magnet (Marconi Picker Polaris 1.0). Axial and sagittal spin-echo T1-weighted images (531/12/2 [TR/TE/NEX]), FSE double echo DP/T2-weighted images (3523/20-120/2, ETL 4), FSE FLAIR images (6000/2100/80/1 [TR/TI/TE/NEX]), and axial diffusionweighted echo planar images (6597/113.4; b value 800$)$ were obtained. After contrast medium administration axial and coronal $\mathrm{T} 1$ images were obtained. All axial images had the same thickness and gap (5.5 and $0.5 \mathrm{~mm}$ respectively). Mean ADC values were calculated within the tumour mass, avoiding cystic and necrotic components of the meningiomas. ADC values were compared to histological results after resection. RESULTS

13 of meningiomas $(29 \%)$ were malignant or atypical with mean ADC values of $0,96 \pm 0,118 \times 10-3 \mathrm{~cm} 2 / \mathrm{sec}$, with range of $0,81-$ $1,25 \times 10-3 \mathrm{~cm} 2 / \mathrm{sec} .32(71 \%)$ were typical with mean ADC values of $1,01 \pm 0,203 \times 10-3 \mathrm{~cm} 2 / \mathrm{sec}$, with range of $0,79-1,84 \times$ $10-3 \mathrm{~cm} 2 / \mathrm{sec}$. The difference in mean ADC values between the two groups was not statistically significant $(p=0,475$, Test $U$ Mann-Whitney).

CONCLUSION

in our population the ADC values did not discriminate between atypical/malignant meningiomas and typical meningiomas.

\section{LP04}

\subsection{0-12.30 - Tumors II}

\section{LP04:19:1}

\section{SEIZURE-INDUCED REVERSIBLE OR PARTIALLY REVERSIBLE BRAIN MRI SIGNAL ABNORMALITIES: PERSONAL EXPERIENCE ON 19 CONSECUTIVE PATIENTS}

\author{
A. Cianfoni ${ }^{1}$, A. Cerase ${ }^{2}$, M. Caulo ${ }^{3}$, G. Della Marca ${ }^{4}$, \\ T. Tartaglione ${ }^{1}$, G. Di Lella ${ }^{1}$, C. Colosimo ${ }^{1}$ \\ ${ }^{1}$ Dept. Bio-Images Radiological Sciences - Catholic University - \\ Policlinico Gemelli, Rome, Italy; ${ }^{2}$ Neuroradiology Uoc - Azienda \\ Ospedaliera Universitaria Senese, Policlinico Santa Maria alle \\ Scotte, Siena, Italy; ${ }^{3}$ Dept. of Radiology - Itab - University G. \\ D'annunzio, Chieti, Italy; \\ 4 Dept. Neurology - Catholic University - Policlinico Gemelli, \\ Rome, Italy
}

Retrospective review of MRI and clinical charts of 19 consecutive patients, 8 males, 11 females, age range 5 months-78 year old, average 58 year old, studied in three academic institutions, during five years, without clinical and laboratory evidence of encephalitis, showing transient or at least partially resolving brain MRI signal abnormalities following seizures. MRI were performed with 1.5 $\mathrm{T}$ magnets, including FSE T1-, T2-W, FLAIR and contrast- enhanced images; in 9 cases DWI was performed. All patients underwent serial MR imaging, with imaging follow-up duration between 3 and 72 months

In our series we observed single and multiple, unilateral and bilateral, reversible and partially reversible, focal signal abnormalities, in the following locations: hippocampus/mesial temporal lobe (11), non-hippocampal cortex (6), deep grey nuclei (3), corpus callosum (1), cerebellum (1).The lesions were better visible on FSE and FLAIR T2-W images, and none showed contrastenhancement; among 9 patients imaged with DWI, 3 showed restricted diffusion at presentation. All the abnormalities had some degree of swelling and mass effect in the acute phase. The resolution, at least partial, of the focal signal changes was observed at follow-up imaging obtained between 25 and 60 days. The abnormality resolution was complete in $14 / 19$ cases, and partial with residual gliosis or encephalomalacia in $5 / 19$

Transient neuroimaging abnormalities after seizure, of unknown pathophysiology, have been reported, especially in the hippocampus. We report a series of patients with a wide range of possible appearance of seizure-induced MRI signal changes of the brain. These changes have variable and non-specific features, and can simulate a variety of diseases; comparison, when available, with baseline studies, and imaging follow-up are strongly recommended for a correct differential diagnosis

This study illustrates the various possible appearance of seizureinduced brain MR abnormalities and increases the awareness of these lesions in patients after seizures, to avoid false diagnosis of lesional epilepsy

\section{LP04:19:2}

\section{MRI APPARENT DIFFUSION COEFFICIENT \\ VALUES IN THE DIFERENTIAL DIAGNOSIS BETWEEN MEDULLOBLASTOMAS AND EPENDYMOMAS}

M. Galvez, E. Bravo, C. Okuma, R. Rivera, M. Peldoza, D. Maldini, E. Torche, C. Silva, R. Chiorino, G. Sordo, J. Cordovez, L. Badilla Institute of Neurosurgery Dr. Asenjo, Santiago, Chile

\section{INTRODUCTION}

Medulloblastomas and Ependymomas are the most frequent posterior fossa tumors in the pediatric population. Imaging differentiation is difficult with traditional MRI.

\section{OBJECTIVES}

To determine the value of apparent diffusion coefficient (ADC) in characterize medulloblastomas and ependymomas and their histological features.

\section{METHODS AND MATERIALS}

We obtained magnetic resonance imaging studies and measured the ADC in 25 children (aged 1.9 to 16.0 years) prior to surgical resection: 15 medulloblastomas and 10 ependymomas, all of them with histological diagnosis. ADC values were contrasted with measurements from histological slices (cell count, nucleuscytoplasm ratio). 


\section{RESULTS}

Patients with medulloblastomas had lower ADC values than patients with ependymomas $(811 \pm 118 \mathrm{~mm} 2 / \mathrm{s}$ and $1217 \pm$ $240 \mathrm{~mm} 2 / \mathrm{s} ; \mathrm{p}<0.001)$. An inverse correlation was observed between ADC and cell count $(\mathrm{r}=-0.698 ; \mathrm{p}<0.01)$ and $\mathrm{ADC}$ and nucleus-cytoplasm ratio $(\mathrm{r}=-0.684 ; \mathrm{p}<0.01)$.

Using a threshold for ADC values of 1000 , sensitivity was $90 \%$ and specificity $87 \%$ for differentiating ependymomas from medulloblastoma.

\section{CONCLUSION}

We demonstrated significantly different ADC values in medulloblastomas and ependymomas and their possible causes. ADC could be a useful tool for posterior fossa tumor characterization.

\section{LP04:19:3}

\section{PSEUDOTUMOR CEREBRI: WHAT IS NORMAL?}

A. Rohr ${ }^{1}$, G. Reimann ${ }^{1}$, K. Alfke ${ }^{1}$, C. Riedel ${ }^{1}$, S. Ulmer $^{1}$, T. Bartsch ${ }^{2}$, L. Dörner ${ }^{3}$, O. Jansen ${ }^{1}$

${ }^{1}$ UKSH Section Neuroradiology, Kiel, Germany; ${ }^{2}$ UKSH Clinic of Neurology, Kiel, Germany; ${ }^{3}$ UKSH Clinic of Neurosurgery, Kiel, Germany

\section{PURPOSE}

Changes in intracranial pressure lead to variation in the width of the optic sheaths, to deformation of the pituitary and to a change in the size of the cavum of Meckel. The anatomy of these structures thus serves as a diagnostic marker for the evaluation of intracranial pressure. The purpose of this study was to determine their normal size and investigate wether age related changes occur. METHODS

113 persons (18 to 86 years) were prospectively enrolled and divided into three equally large subsets ((1): $18-40$ years, $n=37$; (2): 4162 years, $n=38$; (3): $63-86$ years, $n=38)$ ). 3T MRI was used to measure the diameter of the optic nerve and the perioptic sheath in 4 different locations. Width and height of the pituitary gland and the cavum of Meckel were recorded. Exclusion criteria were headache or other signs or symptoms of intracranial hyper- or hypotension.

RESULTS

The optic nerve showed little variance (diameter $2-2.5 \mathrm{~mm}$ ). The width of the fluid filled perioptic nerve sheath varied in the intraorbital course, being greatest directly behind the eyeball $(3,2 \mathrm{~mm}$; SD 0,57$)$ and narrowing to about $2,4 \mathrm{~mm} 2 \mathrm{~cm}$ dorsal to the eyeball (SD 0,45 ). No significant change of these parameters was seen with aging. The mean volume of the pituitary was $417 \mathrm{~mm}^{\wedge} 3$ (SD 167). It decreased from the younger subset of patients (subset 1) from $459 \mathrm{~mm}^{\wedge} 3$ (SD $171)$ to $371 \mathrm{~mm}^{\wedge} 3$ (SD 171) in subset 3 . The mean area of the cavum of Meckel in the coronal plane was $49,8 \mathrm{~mm}^{\wedge} 2$ (SD 12,5). It increased slightly from $47,3 \mathrm{~mm}^{\wedge} 2(\mathrm{SD} 13,3)$ in subset 1 to $52,3 \mathrm{~mm}^{\wedge} 2$ (SD 12,4) in subset 3.

\section{CONCLUSION}

The size of the pituitary and the cavum of Meckel were found to be age-dependent. Because the optic nerve and nerve sheath did not show age dependency, they might be the easiest parameters used for the assessment of intracranial pressure changes.

\section{LP04:19:4}

\section{MRI AND MR-VENOGRAPHY IN PATIENTS WITH PSEUDOTUMOR CEREBRI: REVERSIBILITY OF INTRACRANIAL VENOUS SINUS STENOSES AND FOLLOW UP}

A. Rohr $^{1}$, K. Alfke ${ }^{1}$, T. Bartsch ${ }^{2}$, L. Dörner ${ }^{3}$, O. Jansen ${ }^{1}$

${ }^{1}$ UKSH Section of Neuroradiology, Kiel, Germany;

${ }^{2}$ UKSH Clinic of Neurology, Kiel, Germany; ${ }^{3}$ UKSH Clinic of Neurosurgery, Kiel, Germany

\section{PURPOSE}

To distinguish between idiopathic and secondary intracranial hypertension by the means of cranial MRI and MR-venography. METHODS

14 Patients with typical signs and symptoms of pseudotumor cerebri were examined. Cranial MRI with special emphasis on the optic nerve sheaths and the pituitary and phase contrast venous MRangiography were performed when CSF pressure was documented to be increased on lumbar puncture. A second MR examination was done after CSF pressure was reduced to normal levels. Treatment included oral acetazolamid $(8 / 14)$ or surgical shunting procedures $(n=6 / 14)$. In the follow up of the patients (1-22 months, mean 6 months) clinical data and MRI-findings were correlated.

\section{RESULTS}

The intital MRI / MR venography in all patients showed intracranial venous stenoses. Radiological signs of increased intracranial pressure such as widening of the optic sheaths and "empty sella" were in all patients in accordance with elevated pressure on lumbar puncture. In 10/14 patients, CSF diversion lead to partial or total abolishment of intracranial sinus stenoses, indicating that intracranial pressure induced the stenosis and not vice versa. In these patients, signs of elevated intracranial pressure were reversed. Follow up MRI was in accordance with clinical signs. In 4/ 14 patients, venous sinus stenoses and signs of increased intracranial pressure prevailed.

\section{CONCLUSIONS}

MRI and MR-venography prior to and after CSF diversion can help diagnosing pseudotumor cerebri and distinguishing between idiopathic and secondary intracranial hypertension. It can be used as a valuable non-invasive tool in the follow up of these patients.

\section{LP04:19:5}

\section{SEGMENTATION OF PHASE CONTRAST MR VENOGRAPHY IN PSEUDOTUMOR CEREBRI}

A. Rohr ${ }^{1}$, J. Bindeballe ${ }^{1}$, C. Riedel ${ }^{1}$, K. Alfke ${ }^{1}$, $\overline{\text { M. Tietke }}^{1}$, S. Ulmer ${ }^{1}$, L. Dörner ${ }^{2}$, T. Bartsch ${ }^{3}$, O. Jansen ${ }^{1}$

${ }^{1}$ UKSH Section of Neuroradiology, Kiel, Germany;

${ }^{2}$ UKSH Clinic of Neurosurgery, Kiel, Germany; ${ }^{3}$ UKSH Clinic of Neurology, Kiel, Germany 


\section{PURPOSE}

Bilateral transverse sinus stenoses are regular findings in MR Venography (MRV) in patients with pseudotumor cerebri (PC). Their pathophysiological role is still unclear. To evaluate volume changes of the venous sinuses we performed volumetries in MRVs in patients with $\mathrm{PC}$ before and after lumbar puncture.

\section{METHODS}

Twelve patients with PC were included, nine of them with idiopathic and two with secondary intracranial hypertension. Cranial MRI and phase contrast MR angiography (PCA) were done before and after lumbar puncture. Signs of intracranial hypertension such as widening of the perioptic sheaths and changes of the intracranial sinuses were documented. PCA data of the sinus were segmented (ITK-SNAP V.1.4, University of North Carolina at Chapel Hill) as a whole.The volumes of the superior sagittal sinus, the straight sinus and the transverse sinuses were determined individually and volume changes before / after lumbar puncture were recorded.

\section{RESULTS}

Bilateral transverse sinus stenoses were seen in all initial PCAs. These stenoses resolved fully or partially after lumbar puncture. Also, signs of increased intracranial pressure such as widening of the optic sheaths resolved at least partially by lowering the intracranial pressure. Volumetry of the venous sinus after lumbar puncture documented enlargement not only of the transverse sinus volume (mean enlargement of $65 \%$ from initially $1270 \mathrm{~mm}^{\wedge} 3$ ) but also significant volume enlargement of the superior sagittal sinus and the straight sinus (mean enlargement of $20 / 40 \%$ from 4481 / $397 \mathrm{~mm}^{\wedge} 3$ ).

\section{CONCLUSIONS}

This is the first time that MR volumetry of the intracranial venous sinus was done. It was shown that increased intracranial pressure is not only associated with transverse sinus stenoses but that reversible volumetric changes occur in all parts of the venous sinus system in patients with PC. Volumetry thus expands the diagnostic repertoire and gives rise to considerations concerning the pathophysiology of the pseudotumor syndrome.

\section{LP05}

15.50-16.30 - Session XX - Pediatrics: metabolic diseases

\section{LP05:20:1}

\section{LONGITUDINAL MRI FINDINGS IN THREE GIRLS WITH PELIZAEUS-MERZBACHER PHENOTYPE DUE TO DISTINCT MOLECULAR MECHANISMS}

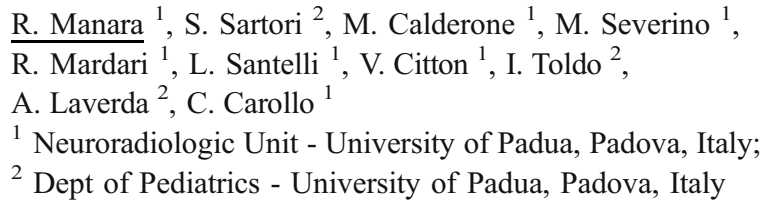

${ }^{2}$ Dept of Pediatrics - University of Padua, Padova, Italy

\section{BACKGROUND}

Pelizaeus-Merzbacher disease (PMD) is the best-characterized hypomyelinating leukoencephalopathy, caused by mutation of Xlinked PLP1 gene, encoding the two major CNS myelin proteins in olidodendrocytes. PMD typically affects males, but female can be clinically affected, often due to unfavourable skewed Xinactivation.

Mutations in a gene that encodes a member of the gap-junction protein family, GJA12, were recently found in males and females with autosomic recessive Pelizaeus-Merzbacher like disease (PMLD), phenotypically indistinguishable from PMD. However, in the majority of cases, the genetic basis of PMLD remains unknown.

PURPOSE

to define possible neuroradiological genotype-phenotype correlation and identify potentially peculiar aspects in three PMLD females.

\section{METHODS}

8 brain MR scans, performed at clinical onset and on follow-up, in three girls with PMLD, due to distinct molecular mechanism (case 1: PLP1 triplication caused by Xq-duplication; case 2: GJA12 mutation; case 3 : undefined genetic basis) were analyzed by two neuroradiologists.

\section{RESULTS}

Patient 1 (16 month-old) showed a severe brain atrophy with a thin corpus callosum and diffuse hypomyelination, more evident on sovratentorial regions; on follow-up MRI, 2 years later, a mild improvement of myelination was observed. Patient 2 ( 3 year-old) presented a diffuse hypomyelination with relative sparing of cortical-spinal tract, which remained unchanged in a subsequent MRI follow-up at 5 years. Patient 3 (10 month-old) had a diffuse hypomyelination which progressively worsened in subsequent follow-up at $1.5,3.5$ and 6 years.

\section{CONCLUSIONS}

Although PMLDs show subtle differences on longitudinal MR studies in comparison to PMD due to PLP defect, a strict genotype-phenotype correlation is still lacking.

\section{LP05:20:2}

\section{DIFFUSION WEIGHTED IMAGING (DWI) AND WHITE MATTER ABNORMALITIES (WMA) IN EARLY-TREATED PATIENTS WITH PHENYLKETONURIA (PK)}

V. Citton ${ }^{1}$, R. Manara ${ }^{1}$, A. Burlina ${ }^{2}$, A. Burlina ${ }^{3}$, N. Mainini ${ }^{3}$, M. Severino ${ }^{1}$, R. Mardari ${ }^{1}$, L. Santelli ${ }^{1}$, M. Ermani ${ }^{2}$, E. Zanonato ${ }^{3}$, C. Carollo ${ }^{1}$

${ }^{1}$ Neuradiologic Unit - University of Padua, Padova, Italy;

2 Dept. of Neurological Science - University of Padua, Padova, Italy; ${ }^{3}$ Dept. of Paediatrics - University of Padua, Padova, Italy

\section{BACKGROUND}

White matter abnormalities found in PKU early-treated patients on T2 images and DWI have been related to metabolic-induced intramyelinic cytotoxic oedema. 


\section{PURPOSE}

To evaluate the contribution of DWI in understanding the nature of WMa through a 4-year follow-up study in young patients with early-treated PKU.

METHODS

24 patients (21 with classical and 3 with mild PKU, mean-age 19 years, range 10-29 years) were prospectively clinically and biochemically evaluated; all these patients underwent conventional and diffusion weighted imaging with $1.0 \mathrm{~T}$ scanner. WMa were classified according Thompson's scale on T2 images and the scores were compared with DWI findings. Apparent Diffusion Coefficient (ADC) values were measured in 16 different white matter brain areas in PKU patients and age-matched controls. White matter anisotropy was studied in the corpus callosum on sagittal and axial slices.

\section{RESULTS}

Even if early-treated, all patients older than 13 years presented T2 and DWI periventricular hyperintensities. DWI was able to distinguish typical PKU lesions from other causes of white matter T2 hyperintensity such as terminal areas of myelination. WMa were more evident in parieto-occipital periventricular zones. ADC values in PKU patients were significantly lower in anterior and posterior part of the centrum semiovale $(690 \times 10$ $-6 \mathrm{~mm} 2 / \mathrm{sec}$ and $686 \times 10-6 \mathrm{~mm} 2 / \mathrm{sec}$ respectively, $\mathrm{p}<0,01)$, in the genu and splenium of the corpus callosum $(741 \times 10-6 \mathrm{~mm} 2 /$ sec and $815 \times 10-6 \mathrm{~mm} 2 / \mathrm{sec}$ respectively, $\mathrm{p}<0.05)$; other areas did not reach a statistically significant difference in comparison to controls. Although ADC values in the corpus callosum were reduced, anisotropy appeared substantially preserved. ADC values did not change during the 4-year MRI follow-up.

CONCLUSIONS

DWI has shown to be very sensitive in detecting WMa in early-treated PKU teenage patients. The long persistence of WMa appears in contrast with the hypothesis of the cytotoxic nature of DWI abnormalities. Moreover, the preserved anisotropy of the affected white matter suggests that other causes (e.g. intracellular phenylalanine accumulation) could partly explain the observed DWI abnormalities.

\section{LP05:20:4}

\section{ENHANCING CRANIAL NERVES AND CAUDA \\ EQUINA: A RARE PRESENTING SIGN \\ OF METACHROMATIC LEUKODISTROPHY AND KRABBE DISEASE}

G. Morana, R. Biancheri, M. Marazzi, A. Pessagno, C. Gandolfo, A. Rossi

G. Gaslini Children's Research Hospital, Genoa, Italy

Metachromatic leukodistrophy (MLD) and Krabbe disease (KD) are lysosomal storage disorders characterized by central and peripheral nervous system involvement, including leukodystrophy and polyneuropathy. While brain MR imaging findings of these two entities are largely described, very few data are available regarding peripheral nerve involvement on imaging. We report the first two cases, to our knowledge, of late infantile MLD and early infantile KD presenting with extensive cranial nerve and cauda equina gadolinium enhancement in the absence of typical brain MR imaging findings. Peripheral cranial nerve and caudal nerve root enhancement may precede typical intracranial findings, and cerebrosidoses should be included in the differential diagnosis of enhancing peripheral nerve roots.

\section{LP05:20:5}

\section{RADIOLOGICAL SPECTRUM OF POSTERIOR REVERSIBLE ENCEPHALOPATHY SYNDROME IN PEDIATRIC PATIENTS FOLLOWING ALLOGENEIC BONE MARROW TRANSPLANTATION}

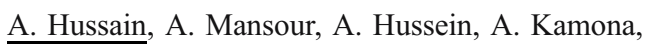
H. Al-Ali, M. Swaidan, M. Sarhan

King Hussein Cancer Center, Amman, Jordan

\section{PURPOSE}

To study the radiological spectrum of Posterior Reversible Encephalopathy Syndrome (PRES) following allogenic Bone marrow transplant (BMT) in pediatric patients.

\section{PATIENTS AND METHODS}

We reviewed charts for pediatric patients who developed PRES following allogenic BMT at King Hussein Cancer Center (KHCC) between January 2003 and February, 2007.

\section{RESULTS}

Out of 110 pediatric patients received allogenic BMT, 11 patients (10\%) developed clinical and radiological features consistent with PRES .The median age of the affected patients was 8 years. All patients developed visual disturbances, headache, seizure and elevated blood pressure.

MRI studies done within 48 hours from the clinical onset, depicted abnormal hyperintense signals in FLAIR sequence in all patients. Ten patients had symmetrical changes. Combined cortical and subcortical affection were noticed in 8 patients. Subcortical involvement in 2 patients. .Half of the cases demonstrated variable degree of gyriform enhancement . The occipital lobe involvement seen in all patients, the parietal lobe was involved in 9 cases, temporal lobe in 6 cases, frontal lobe in 6 patients, periventricular region in 3 patients, cerebullar hemispheres in 2 cases and lastly, brain stem in 1 patient. Two patients underwent additional diffusion weighted images, after installation of new MRI unit, illustrating a cytotoxic edema in one patient with no residual abnormality in the follow up images.

Complete radiological resolution was observed in 10 patients. Only one patient had residual involvement of the right occipital lobe and experienced relapse of PRES with deterioration of neurological status that led to death.

CONCLUSION

PRES is a relatively frequent complication of pediatric allogenic BMT. PRES affects both anterior and posterior cerebral circulations .Combined cortical and subcortical involvement appeared to be the predominant pattern. Post contrast enhancement seen in significant proportion of patients. Although limited to two patients, diffusion weighted images did not predict the irreversibility. 


\section{September 23, 2007 Maestrale Room}

\section{MA09}

\subsection{0-11.05 - Interventional: spine}

\section{MA09:21:1}

\section{ANTERIOR MEDIAL TRANSORAL C2 VERTEBROPLASTY}

\section{G. Callari, S. Vallone}

Neuroradiology of Imperia - Ponente Ligure Neurosciences Department, Imperia, Italy

We present the case of a 63 years old man with a $\mathrm{C} 2$ metastasis and few months life expectancy who was independent at the time of diagnosis. In accordance with oncologists and radiotherapists, under antibiotic prophylaxis and general anesthesia, C2 vertebroplasty with an anterior medial transoral approach was performed, followed by radiotherapy. A satisfactory clinical result, consisting in pain relief and avoidance of progression to vertebral collapse and spinal cord compression, was obtained. We suggest that, even in compromised patients with short life expectancy with a C2 metastasis, difficult to access from a technical point of view, vertebroplasty should be performed.

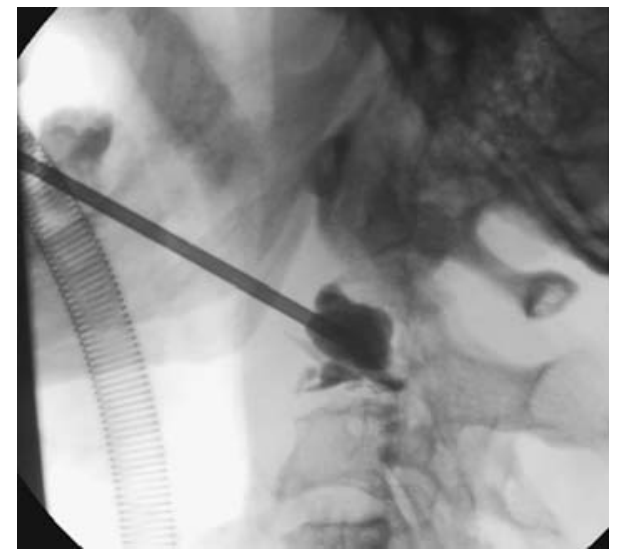

\section{MA09:21:2}

\section{PERCUTANEOUS VERTEBROPLASTY: OUR CENTER 30 MONTHS EXPERIENCE}

\section{G. Callari, A. Arrigo, A. Friedmann, S. Vallone}

Neuroradiology of Imperia - Ponente Ligure Neurosciences Department, Imperia, Italy

We reviewed our center 30 months experience (from July 2004 to December 2006) on Percutaneous Vertebroplasty performed in
120 consecutive patients looking at success rate and complications.

Our results confirm that percutaneous PMMA vertebroplasty is a safe and effective method to treat vertebral compression fractures. A significant improvement in pain can be expected for most patients with a low complication rate.

We suggest that Percutaneous Vertebroplasty can be safely performed even in small but well equipped Hospitals, if adequately trained physicians select patients, paying a particular attention to indication and contraindication for the procedure, and use a good quality fluoroscopic visualization system for cement injection monitoring.

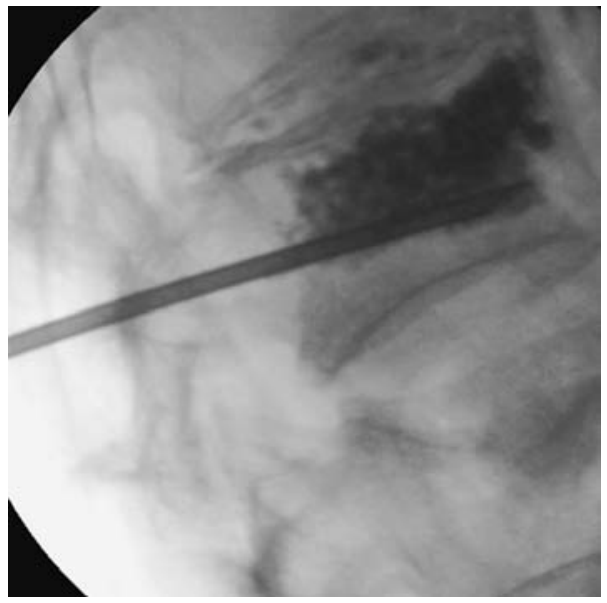

\section{MA09:21:3}

\section{NUCLEOPLASTY: MINIMALLY INVASIVE TREATMENT FOR SINTOMATIC DISC HERNIATION}

\section{E. Pierangeli, N. Gigante}

Ospedale Ss. Annunziata, Taranto, Italy

Nucleoplasty ${ }^{\circledR}$ : minimally invasive treatment for sintomatic disc herniation.

E. Pierangeli, N. Gigante

Neurosurgery Division, "SS. Annunziata " hospital, Taranto (Italy)

\section{INTRODUCTION}

Nucleoplasty ${ }^{\circledR}$ is a minimally invasive tecnique for the treatment of disc protrusion and sintomatic disc herniation without expulsion of the nucleus. Nucleoplasty is based on the concept that the intervertebral disc is like a closed hydraulic sistem in which even a little removal of material creates a strong reduction of the hydraulic pressure inside the disc. Basic condition to perform Nucleoplasty is the integrity of the anulus. The percutaneous introduction of a wire, the spine WAND, inside the interevrtebral disc allows the creation of a low temperature field of radiofrequency (about $70^{\circ}$ ) where ionized particles have energy strog enough to break the links between the collagen molecules near the top of the wire. Simple molecules and inert gas (id e. oxygen, hydrogen, and hydrogen dioxide) are the residui of Nucleoplasty. 
Aim of this study is to evalue the safety and the effectiveness of this tecnique, performed under local anesthesia, in patient with sintomatic disc herniation.

\section{MATERIALS AND METHODS}

from May 2005 to December 2006, 32 Nucleoplasty have been performed in 30 patients, 15 males and 15 females, in the Neurosurgery Division of "S.S. Annunziata" hospital in Taranto. Before intervention, all the patients underwent drug and phisical therapies without good results or with a narrow pain relief. All interventions have been performed under local anesthesia (Carbocaina 2\%) and radioscopic control allowing the patients an easy recovery. 31 Nucleoplasties have been performed in the lumbar spine: L2-L3 (1 patient), L3-L4 (4 patients), L4-L5 (19 patients) and L5-S1 (7 patients); 1 in the cervical spine (C5-C6). Two patients underwent Nucleoplasty in two different levels in the same surgical session.

\section{RESULTS}

in six of the 29 patients with lumbar Nucleoplasty ${ }^{\circledR}$ the pain reappeared, so 3 of them were submitted to microdiscectomy two months later due to the contemporary presence of lumbar stenosis and a low pain relief that made particulary difficult the management of the same pain. Two patients report a mild reduction of the pain. The pain is disappeared in the remaining patients. All the patients were discharged two days after Nucleoplasty.

\section{CONCLUSIONS}

In well selected patients, Nucleoplasty can allow an important pain reduction despite very low surgical risks and recovery. The cost of the single-use instruments remains a restricting factor.

\section{MA09:21:4}

\section{MINIMALLY INVASIVE TREATMENT FOR SPINE METASTASIS: TEN YEARS EXPERIENCE}

E. Pierangeli, N. Gigante

Ospedale Ss Annunziata, Taranto, Italy

\section{PURPOSE}

the increasing development in diagnostics has maken the diagnosis of spine metastasis in cancer patients (pz) more and more frequent. 18/ 20.000 new cases of spine metastasis are diagnosed every year in USA and about $4-5 \%$ of diagnosed malignant tumors will present one or more metastasis to the spine. The pain, sometimes of severe entity, is always present and compromises the quality of life of the patients, already strongly compromised by the cancer.

Aim of this study is the analisis of the results in patients undergone to minimally invasive surgical treatments for spine metastasis in the Neurosurgery Division of "SS. Annunziata" hospital in Taranto during the last ten years.

Methods: from January 1997 to Febrary 200763 Kyphoplasties have been performed in 40 patients, 21 males e 19 females, suffering for spine metastasis. The average age was 67,4 years, 72 years in males (54-89) and 62,1 in females (38-81). The most frequent metastasis origin has resulted breast cancer (10 patients), lung cancer ( $7 \mathrm{pz}$ ) and multiple myeloma $(10 \mathrm{pz})$. We have performed Kyphoplasty in $16 \mathrm{pz}$
(3M e 13F), bilateral laminectomy in $7 \mathrm{pz}(6 \mathrm{M} \mathrm{e} 1 \mathrm{~F})$ and monolateral laminectomy in $3 \mathrm{pz}(1 \mathrm{M}$ e $2 \mathrm{~F})$, laminectomy e vertebroplasty in $2 \mathrm{~F}$, monolateral laminectomy and Kyphoplasty in $10 \mathrm{pz}(9 \mathrm{M}$ e $1 \mathrm{~F})$, somatectomy and Kyphoplasty in 2 pz (1M e 1F), somatectomy and ADD distractable cage in $1 \mathrm{M}$. All patients were submitted to pre- and post-operative radiological and MRI investigations. The approach has always been posterior except in 3 patients in which an anterior approach was required for somatectomy

RESULTS

in more than $90 \%$ of the patients the pain was strongly reduced or disappeared in corrispondence of the operated vertebra.

In two patients, suffering for dorsal metastasis from lung cancer, the pain was still persistant in half the thorax with root distribution due to neoplastic infiltration of the nerve tissue. In this cases the prosecution of morphine therapy was required. No neurological worsening was noted.

Conclusions: the analysis of clinical and radiological follow-up has given good results. Our experience suggests that, when clinical and radiological conditions of the patients are favourable, a minimally invasive surgical treatment has to be always and early proposed to try to improve their quality of life.

\section{MA09:21:5}

\section{LONG-TERM CHANGES AFTER ANTERIOR CERVICAL DISCECTOMY AND FUSION: DYNAMIC MRI WITH HIGH RESOLUTION 3D T2-IMAGING}

\author{
$\underline{\text { L. Gerigk }}^{1}$, T. Bostel ${ }^{1}$, A. Hegewald ${ }^{2}$, C. Thomé ${ }^{2}$, \\ J. Scharf $^{1}$, C. Groden ${ }^{1}$ \\ ${ }^{1}$ University Hospital Mannheim, Neuroradiology, Mannheim, Ger- \\ many; ${ }^{2}$ University Hospital Mannheim, Neurosurgery, Mannheim, \\ Germany
}

\section{PURPOSE}

Patients who have undergone anterior cervical discectomy and fusion (ACDF) are prone to degenerative changes in adjacent segments. We evaluate a new combination of flexion-extension MR-imaging and a high-resolution 3D T2-weighted sequence (SPACE) for visualization of morphologic changes five years after surgery and compare different disc replacement materials, i.e. autologous bone grafts and titanium cages.

\section{METHODS}

24 patients (median age $51.5 \mathrm{y}, 11 \mathrm{f}, 13 \mathrm{~m}$ ) were examined after a median interval of 62.5 months from ACDF, 14 with titanium cage and 10 with autologous bone graft. Using a commercially available positioning device (NeuroSwing), flexion was performed up to $45^{\circ}$ and extension up to $40^{\circ}$ or until patient discomfort and monitored using a single-slice, real-time true-FISP sequence (6 mm, TR 704, TE $1.3 \mathrm{~ms}$, Matrix $256 \times 207$, FoV $22 \mathrm{~cm})$. Imaging in the end positions consisted of a sagittal T2 SPACE-sequence ( $0.9 \mathrm{~mm}$ isotropic voxels, TR 1770 , TE $186 \mathrm{~ms}$, Matrix $320 \times 318$, FoV $28 \mathrm{~cm}$ ) and an axial true-FISP sequence ( $3 \mathrm{~mm}$ slices, TR 194, TE $1.9 \mathrm{~ms}$, Matrix $256 \times 256$, FoV $22 \mathrm{~cm}$ ). Spinal stenosis was graded into $0=$ normal width of the spinal canal, 1 = partial, 2 = complete obstruction of the anterior and / or posterior subarachnoid space and $3=$ spinal cord compression. 


\section{RESULTS}

Compared to primary axial T2 true-FISP slices, axial reconstructions of the T2 SPACE-sequence were found to be clearly less affected by metal artifacts with the additional benefit of multiplanar and transforaminal reconstructions, allowing for assessment of foraminal narrowing. Relevant narrowing of the spinal canal in flexion, i.e. from grade 1 to 2 or from 2 to 3 , was found in $9 / 24$ patients $(37.5 \%), 3 / 14(21.4 \%)$ with titanium cages and $6 / 10$ $(60 \%)$ with autologous bone. In extension, a relevant aggravation, i.e. from grades 0 or 1 to 2 , was found in $12 / 24$ patients $(50 \%), 5$ / $14(35.7 \%)$ with titanium and 7/10 (70\%) with bone. CONCLUSIONS

The SPACE-sequence allows high resolution imaging of the cervical spine and is especially advantageous in the presence of metal implants. Combined with dynamic MRI, a good visualization of long-term changes after ACDF can be achieved. We found relevant narrowing of the spinal canal for flexion in $37.5 \%$ and extension in $50 \%$ of the patients. For both positions, we found titanium implants to be associated with fewer changes in the adjacent segments than autologous bone.

\section{MA09:21:6}

IMPACT OF UPRIGHT, MULTI-POSITION, FUNCTIONAL MRI (FMRI) ON THERAPEUTIC STRATEGIES IN CERVICAL SPINE.

\author{
J. Elsig $^{1}$, D. Kaech ${ }^{2}$, J. Naxera ${ }^{1}$ \\ ${ }^{1}$ Fmri Zentrum, Zurich, Switzerland; ${ }^{2}$ Ksgr, Chur, Switzerland
}

\section{INTRODUCTION}

The diagnostic accuracy in spinal imaging has been improved since the introduction of MRI. However some lesions of the cervical spine and cord still remain unexplained. Since functional imaging under weight bearing conditions and during kinetic maneuvers acquired in a top-front open, upright, multi-position unit (fmri) has become available, several previously undiagnosed or underestimated dynamic components of degenerative and posttraumatic changes of the cervical spine could be depicted.

\section{METHOD}

Patients who developed pain and/or neurological deficit following surgery or minor trauma of their cervical spine but having negative recumbent MRI were studied in sitting upright neutral, flexionextension and some in rotation. The images acquisition is made in a patient's triggering position.

\section{DISCUSSION}

Illustrative cases let us relatively perplex as conventional recumbent imaging has missed unstable cervical lesions, e.g. unsuspected dynamic disc herniation sometimes associated with only unilateral instability, dynamic $\mathrm{C} 1 / 2$ stenosis due to missed odontoid fracture, adjacent segment disease. The real potential of this new modality of spine investigation is presently under-recognized.

The personal, familiar and socio-economical benefit of adequate investigation and surgical treatment is evident in case of a clear mechanical lesion.

\section{CONCLUSION}

Investigation of these unexplained pathologies is now possible under weight bearing conditions and the results of upcoming studies will further document the potential of this new functional and dynamic MR. In the near future fmri may become mandatory for the investigation of complex spinal pathologies, especially when no obvious explanation can be derived from conventional recumbent imaging.

\section{MA09:21:7}

\section{EMBOLIZATION OF INTRADURAL VASCULAR SPINAL CORD TUMORS. REPORT OF 5 CASES AND REVIEW OF THE LITERATURE}

G. Rodesch $^{1}$, S. Gaillard ${ }^{1}$, A. Boulin ${ }^{1}$, S. Condette Auliac ${ }^{1}$, $\overline{\text { H. Loiseau }}^{2}$, J. Brotchi ${ }^{3}$

${ }^{1}$ Hopital Foch, Suresnes, France; ${ }^{2}$ Chu Pellegrin, Bordeaux, France; ${ }^{3}$ Hopital Erasme, Bruxelles, Belgium

\section{INTRODUCTION}

Spinal cord tumors (SCT) are best treated by surgery. Vascular SCT represent a special challenge because of the bleeds that may occur during surgery, and the subsequent difficulties in operating these lesions. Embolization may thus be proposed in order to facilitate SCT resection.

\section{METHODS}

The clinical and radiological charts of 5 patients with large solid SCT (4 cervical hemangiobastomas, 1 filum terminale paraganglioma) were retrospectively reviewed. All these hypervascular lesions have been superselectively embolized prior to surgery (4 with NBCA, 1 with particles) .

\section{RESULTS}

Intranidal deposition of glue could be achieved in each of the 4 cases resulting in significant devascularisation of the tumor. Because of arterial tortuosities it was not possible to reach distally a cervical hemangioblastoma that was thus embolized with particles. No permanent complication occurred after embolization. Surgery became possible in each case in improved conditions with minimal blood loss, allowing total (4 cases of hemangioblastomas) or subtotal (1 case of paraganglioma) removal of the tumor. CONCLUSION

Embolization of intradural vascular tumors is a safe procedure if applied according to strict anatomical and technical rules. Whenever possible, NBCA can be considered as a first intention embolus, particles being reserved to cases where selectivity cannot be achieved. Despite its solid aspect after deposition, glue does not hinder surgery but facilitates the manipulation and eradication of the tumor. Thanks to its initial liquid aspect, NBCA deeply penetrates into the tumoral capillary bed and favourizes thus satisfactory devascularization of the lesion. 


\section{MA09:21:8}

\section{PRESURGICAL AND CURATIVE ENDOVASCULAR EMBOLIZATION FOR SPINAL DISEASE IN CHILDREN}

\author{
$\underline{\text { L. Simonetti }}^{1}$, C. Barbara ${ }^{1}$, S. Boriani ${ }^{2}$, A. Gasbarrini ${ }^{2}$, \\ $\overline{\text { M. Leonardi }}^{3}$ \\ ${ }^{1}$ Neuroradiology Maggiore Hospital, Bologna, Italy; \\ 2 Orthopaedics Maggiore Hospital, Bologna, Italy; \\ ${ }^{3}$ Neuroradiology Bellaria Hospital, Bologna, Italy
}

\section{BACKGROUND}

Presurgical embolization of hypervascularized spinal lesions is an important step in partial and en bloc surgical resection. Embolization in children presents specific features linked to the most common histotypes which differ from those in adults. Embolization also offers the possibility of long-term curative treatments.

We describe our cohort of patients up to the age of 16 years treated in the past 48 months.

\section{PATIENTS AND METHODS}

Between January 2005 and February 2007 embolization was performed in 19 patients aged from six to 16 years $(8 \mathrm{~F}$ and $5 \mathrm{M})$. Histotypes included aneurysmal cyst (7 cases), osteoblastoma (6 cases), Ewing's sarcoma (2 cases) aggressive osteoangiomas (2 cases) and metastasis from osteosarcoma ( 2 cases).

There were 31 embolization sessions with a minimum of one (13 patients) to a maximum of five ( 2 patients). Embolization was performed by endovascular approach in 28 sessions and by transcutaneous route under fluoroscopic or CT guidance in three sessions.

Embolic materials included acrylic glue (Glubran) in most cases, but also particles and coils. Coils were used for closure of the vertebral artery in cervical disease in which the artery would have been sacrificed in any case during surgery.

\section{RESULTS AND CONCLUSIONS}

Surgeons deemed embolization extremely useful in all patients operated (15/19).

There were no complications during or after the embolization procedure.

A complete resolution of pain or deficits and CT evidence of regression or recalcification of the lesions were noted in four patients (aneurysmal cysts).

Presurgical closure of the vertebral artery was performed in four cases ( 1 aneurysmal cyst and 3 osteoblastomas).

The embolic materials and approach route were chosen on the basis of the angio-architecture of the pathological circulation.

In conclusion, the embolization of hypervascularized spinal lesions in children is a complex procedure but useful to allow application of the new radical surgical techniques. The procedure proved curative in itself in some patients with aneurysmal cysts.
MA09:21:9

\section{INTEREST OF 3D ANGIOGRAPHY WITH FLAT PANELS IN THE TREATMENT OF SPINAL CORD DURAL ARTERIO-VENOUS FISTULAS AND ARTERIO-VENOUS MALFORMATIONS}

R. Anxionnat, S. Bracard, N. Pinheiro Jr, G. Andrade, A. Lebedinsky, C. Macedo De Freitas, D. Iancu, L. Picard

Department of Diagnostic and Therapeutic Neuroradiology. University Hospital, Nancy, France

\section{PURPOSE}

To evaluate the interest of flat panels 3D angiography in the pretherapeutic evaluation and treatment of spinal cord arterio-venous malformations (AVMs) and dural arterio-venous fistulas (DAVFs) PATIENTS AND METHODS

2D and 3D angiography were performed on an angiographic suite with flat panels (Innova, GE Healthcare) in 4 cases of DAVFs and 4 cases of intramedullar cervical AVMs. 3D unsubtracted angiography was performed for the pre-therapeutic evaluation and immediately after embolization to assess the quality of endovascular treatment. 3D images were analyzed in MIP, VR and using thick multiplanar volume reformations.

\section{RESULTS}

In all DAVFs, analysis of 3D angiography in VR clearly demonstrated the arterial supply, the location of the shunt and the venous drainage. The accurate site of the fistula and its relationship with the dura mater and the bone were clearly seen on axial and coronal reformatted volumes. 3D angiography also helped to find a working view for endovascular navigation and treatment. Three DAVFs were embolized with glue. Immediate non injected post-embolization 3D angiography nicely showed the cast and that it reached the shunt in 2 cases. In one case where surgery was performed in a second time, there was a perfect agreement between 3D angiographic images and operating view. In AVMs, 3D angiography demonstrated precise location of the nidus and helped to differentiate intra and peri-medullar parts. If necessary it was possible to visualize the location of the tip of the microcatheter with regard to the spinal cord thanks to a $3 \mathrm{D}$ reconstruction. After embolization, the quality of treatment was best assessed by $3 \mathrm{D}$ angiography.

\section{CONCLUSION}

3D angiography using flat panels provides highly valuable additional information to $2 \mathrm{D}$ angiography in the pre-therapeutic evaluation of DAVFs and spinal cord AVMs, in the therapeutic decision especially in AVMs and in the quality of endovascular treatment. 
MA10

\subsection{5-12.00 - Spine \& spinal cord}

\section{MA10:22:1}

\section{OPTIMIZATION OF RADIATION DOSE IN CT EXAMINATION OF THE SPINE IN YOUNG PATIENTS WITH SCOLIOSIS. A PHANTOM STUDY.}

K. Abul-Kasim ${ }^{1}$, M. Gunnarsson ${ }^{1}$, P. Maly ${ }^{1}$, A. Ohlin ${ }^{1}$, P. Sundgren ${ }^{2}$

1 University of Lund, Malmö University Hospital, Malmö, Sweden; ${ }^{2}$ University of Michigan Health Systems, Ann Arbor, USA

\section{BACKGROUND}

Patients with scoliosis have been examined in our institution with CT scan consisting of a few sequential slices of the apical and neutral vertebrae (ANV).

PURPOSE

The aim of the study was to explore the possibility to gain a long helical CT scan of the whole region of interest of the vertebral column and at the same time reduce the radiation dose.

MATERIAL AND METHODS

A chest phantom was examined with 16-slice CT scanner. Twelve scans were performed with different radiation doses.

RESULTS

Our study has shown that major parts of the vertebral column can be imaged with the new low dose helical CT protocol, resulting in a $28.8 \%$ reduction in the radiation dose, compared with that of the previously used ANV-CT protocol. The dose reduction system available in the scanner has contributed to $17.1 \%$ of the total dose reduction. In addition, the low dose helical $\mathrm{CT}$ allows detailed morphological evaluation of the spine.

\section{CONCLUSION}

We recommend low dose 3-dimensional helical CT scan instead of the ANV-sequential slice method in the pre- and postoperative examination of patients with scoliosis. We strongly recommend using the dose reduction system available in modern CT scanners.

\section{MA10:22:2}

EVALUATION OF RADIATION DOSE REDUCTION IN THE PRE- AND POSTOPERATIVE EXAMINATION OF SCOLIOSIS USING LOW DOSE 3D HELICAL CT.

K. Abul-Kasim ${ }^{1}$, P. Maly ${ }^{1}$, A. Ohlin ${ }^{1}$, M. Gunnarsson ${ }^{1}$, P. Sundgren ${ }^{2}$

1 University of Lund, Malmö University Hospital, Malmö, Sweden; ${ }^{2}$ University of Michigan Health Systems, Ann Arbor, USA

\section{BACKGROUND}

As scoliosis affects young individuals and new surgical corrective methods require detailed pre-and postoperative CT-evaluation of large segments of the spine, reduction of the radiation dose is becoming an important objective.

PURPOSE

To estimate the radiation dose in patients examined according a low dose 3-D helical CT-protocol imaging long segments of the spine, developed for the purpose in our institution and to compare it with the dose that patients received when previously examined with a few sequential slices of the apical and neutral vertebrae (ANV) protocol.

MATERIAL AND METHODS

Radiation doses in 113 consecutive examinations with low dose CT-scan were compared with those obtained in 15 randomly chosen examinations previously performed according to the ANVprotocol.

RESULTS

The effective dose received from the low dose CT-examination was significantly lower $(0.37 \pm 0.09 \mathrm{mSv}$, mean $\pm \mathrm{SD})$ than that received from the ANV protocol $(0.52 \mathrm{mSv})$.

Conclusion: Using our low dose helical CT-protocol in young individuals with scoliosis the radiation dose is reduced by a third compared with that obtained at examinations according to the "old" ANV protocol. In addition, it allows for detailed morphological analysis of large segments of the spine, which is necessary for the preoperative planning as well as the post-surgical evaluation.

\section{MA10:22:3}

\section{DIFFUSION TENSOR IMAGING AND FIBER TRACTOGRAPHY OF SCIATIC NERVE AND LUMBOSACRAL PLEXUS: ITS ROLE IN PATIENTS WITH SCIATICA}

R. Vadapalli ${ }^{1}$, R. Mulukutla ${ }^{2}$, S. Satish ${ }^{3}$

1 Vijaya Diagnostics, Hyderabad, India; ${ }^{2}$ Manipal Hospitals, Bangalore, India; ${ }^{3}$ Udai clinic and Institute of spine diseases, Hyderabad, India

\section{PURPOSE}

To assess the role of DTI tractography in patients with sciatica with discogenic and Nerve related patholgies on $3 \mathrm{~T}$ MR imaging METHOD AND MATERIALS

33 patients with Sciatica $(n=33)$ and 22 normal controls were included in the study.All patients underwent a comprehensive Backache protocol on a $3 \mathrm{~T}$ Achieva scanner(Phillips medical) with T1,T2 sagittals and axials, with a standard DTI sequence with parameters as follows: TR $8550 \mathrm{msec}$ TE $79 \mathrm{msec}$ Matrix $128 \times$ 128 FOV $26 \times 20 \mathrm{~cm}$ NEX $1 \mathrm{~B}$ value:750;25 directions,slice thickness $\quad 6 / 0 \mathrm{~mm}$.Area of coverage included L2 till S5 ..Data was the proessed on a functool platform to generate FA, Average ADC maps and Tractograms of the plexcus and Region of interest segments of sciatic nerve at and distal to the site of compression. 


\section{RESULTS}

All normal controls excellent visulaization lumbar plexus and its quantitative indices namely a mean FA value of 0.88 . No significant abrupt variation was found in FA in distal and proximal segments. At the site of disc related compression and plexus edema $(n=15)$ a significant loss of FA with associated loss of FA value in distal segments of plexus and intra and extra pelvic sciatic nerve segments. In pyriformis syndrome related nerve compres$\operatorname{sion}(n=4)$ significant loss of FA on ipsilateral side by $>50 \%$ at the compression site and distal ROI In nerve edema secondary to inflammation $(n=2)$ FA loss was localized to site of edema with FA loss in distal ROI $<20 \%$. In pudendal nerve entrapment $(n=1) F A$ loss was localized to the site of edema with distal FA loss CONCLUSION

Diffusion tensor Imaging (DTI) at $3 \mathrm{~T}$ with fibertractography is an effective tool to understand the pathophysiology of plexus and nerve entrapment syndromes and more commonly Sciatica as the nerve injury due to compression may be more diffuse than what is visualized on macroscopic 3 T MR imaging.

\section{MA10:22:4}

\section{T MR NEUROGRAPHY OF SCIATIC NERVE AND LUMBOSACRAL PLEXUS USING DWIBS SEQUENCE IN PATIENTS WITH SCIATICA: A STUDY OF 23 PATIENTS}

$\underline{\text { R. Vadapalli }}^{1}$, M. Raghavdutt ${ }^{2}$, H. Raveesh ${ }^{3}$

${ }^{1}$ vijaya diagnostics and Imaging research, Hyderabad, India; ${ }^{2}$ uday indtitute of Spine diseases, Hyderabad, India; ${ }^{3}$ Manipal Hospitals, Bangalore, India

\section{PURPOSE}

To evaluate the role of MR neurography of sciatic nerve and Lumbosacral plexus at $3 \mathrm{~T}$ using Diffusion weighted imaging with back ground suppression(DWIBS)

METHOD AND MATERIALS

15 normal volunteers and 22 patients with clinical sciatica with age range of 24-57 years M:F ratio of 1:2 were recruited for the study after Informed consent. All patients were imaged on a $3 \mathrm{~T}$ Achieva X series (phillips Medicals)system wih following protocol: T1/T2 sagittal,DWIBS sequence wih following parameters: TR $>5000 \mathrm{msec}$, TE Min_(70 msec),EPI FACTOR-47,HALF SCAN FACTOR:0.645, TI:180 msec,slice orientation:axial,coronal,Number of slices: 60 slice Thickness/gap: $3 \mathrm{~mm} / 0 \mathrm{~mm} ; 28 \mathrm{~cm}$ FOV coverage from L2 till S5 B value-1000 number of direcions-25.All the images were processed with reverse video on and off on he work station generating $3 \mathrm{D}$ mip plexograms.ADC values were calculated then at the level of the maximum by the disc and distal pelvic and exrapelvic sciatic nerves on both sides and results were computed.

\section{RESULTS}

All normal controls showed excellent visualization of the lumbosacral plexus with its branches $(n=15)$. Sciatic nerve in is intra and extra pelvic course was visualized in all controls $(n=15)$ with a mean ADC OF 0.87. Restriced diffusion abnormalities with low ADC with nerve edema was with mean ADC of 0.45 was seen in 17 patients due to disc related compression.Low ADC disal to compression was seen in 9 patients $(n=9)$. Internal pudendal nerve and pyriformis entrapment syndrome $(\mathrm{n}=5)$ patients showed Low ADC of $>50 \%$ at the entrapment site and disal To compression Lower ADC values with a mean ADC of 0.38 and 0.42 respecively

\section{CONCLUSION}

DWIBS (diffusion Weighted Imaging with background suppression) is an emerging technique for excellent visualization of lumbosacral plexus and intra and extra pelvic course of Sciatic nerve and also shows qunatitative estimaion of nerve injury by $\mathrm{ADC}$ at the site of compression and in the downstream peripheral nerve.

\section{MA10:22:5}

\section{ARACHNOID WEB: A CASE REPORT AND LITERATURE REVIEW OF AN UNUSUAL CAUSE OF SYRINGOMYELIA}

\author{
$\underline{\text { P. Lee }}^{1}$, C. Heilman ${ }^{1}$, R. Bhadelia ${ }^{2}$, G. Moonis ${ }^{2}$ \\ ${ }^{1}$ New England Medical Center, Boston, USA; ${ }^{2}$ Beth Israel \\ Deaconess Medical Center, Boston, USA
}

There are many causes of syringomyelia, including Chiari malformations, tumors, trauma, arachnoiditis, arachnoid cysts, and transdural spinal cord herniation. We present a case of a thoracic syringomyelia that was caused by an arachnoid web. Arachnoid web as a cause of syringomyelia is recognized in the neurosurgical literature but is not well known in the neuroradiology literature. A 43 year old male presents with slowly progressive numbness and parethesias of the arms and chest approximately a year following a minor fall while snowboarding. Magnetic resonance imaging revealed syringomyelia extending from C6 through T4.(Figure 1) There was an abrupt transition from the syringomyelia to the normal cord. The patient was taken to surgery, and an intraoperative ultrasound revealed an arachnoid membrane dorsal to the cord which was flapping with CSF pulsations(Figure 2- A.WMV file, to be submitted later). The membrane was resected, with excellent decompression of the cord. After Chiari malformations and tumors are excluded, the main differential diagnosis for an arachnoid membrane would include arachnoid cyst and transdural cord herniation. We propose that an imaging appearance of a band extending to the cord or an abrupt transition from the syringomyelia to the normal cord may be 
suggestive of an arachnoid web. This imaging appearance should alert the neuroradiologist to this potentially treatable cause of syrinx. We will also perform an imaging literature review for our presentation.

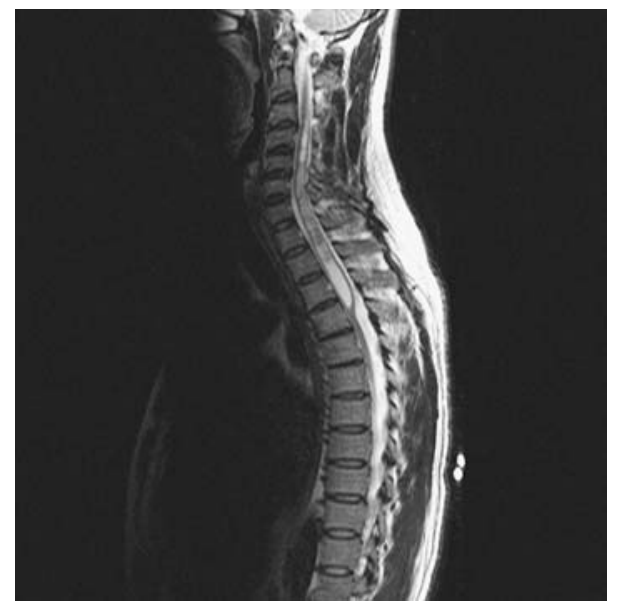

MA10:22:6

\section{COMPARISON OF MULTIHANCE AND OMNISCAN \\ IN THE EVALUATION OF SPINAL VASCULAR \\ ANATOMY WITH MAGNETIC RESONANCE ANGIOGRAPHY}

M. Spampinato, C. Moses, N. Besenski, J. Deveikis, Z. Rumboldt

Medical University of South Carolina, Charleston, USA

\section{PURPOSE}

First-pass three dimensional contrast-enhanced MR angiography (3D CE MRA) allows for a non-invasive evaluation of the intradural spinal vasculature. New gadolinium chelates such as gadobenate dimeglumine (Gd-BOPTA, MultiHance; Bracco Imaging) have been developed which have greater $\mathrm{T} 1$ relaxivity in vivo and intravascular signal enhancement. Our hypothesis was that the use of MultiHance with a 3D CE MRA technique would improve the identification of normal intradural vascular anatomy of the spine compared to Omniscan.

METHODS

We recruited six consecutive subjects who were scheduled to undergo an MRI of the thoracic or lumbar spine at our institution (age range 22-53). Informed consent was obtained. Exclusion criteria were renal failure, pregnancy, clinical suspicion of a vascular abnormality, and presence of surgical hardware. Each patient underwent two spinal contrast-enhanced MRA within two weeks with a double dose of MultiHance or Omniscan (0.20 mmol $/ \mathrm{kg})$.

The following information was gathered from the imaging data: identification of the anterior spinal artery, identification of the artery of Adamkiewicz, and determination of the vertebral level of origin of the artery of Adamkiewicz.

\section{RESULTS}

The anterior spinal artery was identified in $83 \%$ of MultiHanceenhanced and in $50 \%$ of Omniscan-enhanced spinal MRA. The artery of Adamkiewicz was identified in $66 \%$ and $33 \%$ of spinal MRA when MultiHance and Omniscan were used, respectively. The vertebral level of origin of the artery of Adamkiewicz was definitively established in 4 cases using MultiHance-enhanced MRA and only in one case using Omniscan-enhanced MRA.

CONCLUSION

Our preliminary findings indicate that the use of a high relaxivity agent such as MultiHance may significantly improve the identification of normal intradural vessels with a 3D CE MRA technique compared to a traditional gadolinium compound as Omniscan.

\section{MA10:22:7}

OVERVIEW: CURRENT STANDARDS AND TESTING METHODS FOR MR SAFETY AND COMPATIBILITY OF NEUROLOGICAL IMPLANTS AND DEVICES

G. Schaefers

MR:comp GmbH, Gelsenkirchen, Germany

\section{OBJECTIVE}

Magnetic resonance (MR) safety and image compatibility are internationally recognized as important issues especially for neurological implants. The American Society of Testing and Materials (ASTM) standard F2503 provides comprehensive marking requirements. FDA guidelines, first European and International standards contain MR safety and imaging compatibility requirements. Medical devices and items that can be exposed to an MR environment must be basically tested on magnetically induced forces, torques, RF heating, induction of voltages and safe functioning as well as MR image artifacts.

\section{SUBJECTS AND METHODS}

Magnetically induced forces can be measured via the deflection angle test to determine the forces and risk magnitude. Magnetically induced torque aligns a device to the magnetic field and is measured at the magnet isocenter. ASTM F2052 and F2213 provide standard testing methods (e.g. for aneurysm clips, coils, stents).

Radio frequency (RF) induced heating is an important MR safety issue. RF pulses are in the area of $\mathrm{MHz}$ and apply the main amount of heating energy. This topic is complex and multiparameter dependent. Not only device properties like electric conductivity, dimension, etc. have to be considered, but also geometric arrangement (e.g. the configuration and orientation of neurostimulator leads, reinforced drainages, pressure sensors, etc.) relative to the specific MR environment. ASTM F2182 provides a basic test method. Computer simulation of electromagnetic fields, SAR and temperature distribution assist in heating testing. RF pulses and switched gradients can induce voltages in conductive structures. No appropriate standardized test method is available, but is under development. 
The safe functioning is a concern for the MR system and the device respectively. A device must undergo an individual test procedure.

MR imaging artifacts can lead to significant lack of information and thus shall be included in device labeling with results from ASTM F2119 standard test method.

RESULTS

Comprehensive investigation of all interactions and worst-case scenarios is necessary. MR test methods for magnetic force, torque, RF heating and MR artifacts are established. Continuous redefining is required. Further issues have to be examined for standardization.

\section{CONCLUSION}

Standardized MR testing of neurologic medical devices and items is compulsory for minimizing patient risk and providing the MR user (clinician/physicist) with a comprehensive safety labeling as well as guiding manufacturers in development of devices. 